\title{
Silver-Catalyzed Trifluoromethylalkynylation of Unactivated Alkenes with Hypervalent Iodine Reagents
}

Xinkan Yang and Gavin Chit Tsui*

Table of Contents

General Experimental

General Experimental. Unless otherwise noted, reactions were carried out under argon in a $25 \mathrm{~mL}$ round-bottom flask with magnetic stirring. Analytical thin layer chromatography (TLC) was performed with EM Science silica gel 60 F254 aluminum plates. Visualization was done under a UV lamp (254 nm) and by immersion in ethanolic phosphomolybdic acid (PMA) or potassium permanganate $\left(\mathrm{KMnO}_{4}\right)$, followed by heating using a heat gun. Organic solutions were concentrated by rotary evaporation at 23$35^{\circ} \mathrm{C}$. Purification of reaction products were generally done by flash column chromatography with Grace Materials Technologies 230-400 mesh silica gel.

Materials. Anhydrous DMSO and $\mathrm{AgOCOCF}_{3}$ was purchased from J\&K Scientific. Other chemicals for substrates preparation were purchased from Acros, J\&K Scientific, Aldrich and Dikemann.

Instrumentation. Proton nuclear magnetic resonance spectra $\left({ }^{1} \mathrm{H}\right.$ NMR) spectra, carbon nuclear magnetic resonance spectra $\left({ }^{13} \mathrm{C} \mathrm{NMR}\right)$ and fluorine nuclear magnetic resonance spectra $\left({ }^{19} \mathrm{~F} \mathrm{NMR}\right)$ were recorded at $23{ }^{\circ} \mathrm{C}$ on a Bruker 400 spectrometer in $\mathrm{CDCl}_{3}\left(400 \mathrm{MHz}\right.$ for ${ }^{1} \mathrm{H}, 101 \mathrm{MHz}$ for ${ }^{13} \mathrm{C}$ and 376 $\mathrm{MHz}$ for ${ }^{19} \mathrm{~F}$ ) and Bruker 500 spectrometer in $\mathrm{CDCl}_{3}\left(500 \mathrm{MHz}\right.$ for ${ }^{1} \mathrm{H}, 126 \mathrm{MHz}$ for ${ }^{13} \mathrm{C}$ and $470 \mathrm{MHz}$ for ${ }^{19} \mathrm{~F}$ ). Chemical shifts for protons were reported as parts per million in $\delta$ scale using solvent residual peak $\left(\mathrm{CHCl}_{3}: 7.26 \mathrm{ppm}\right)$ or tetramethylsilane $(0.00 \mathrm{ppm})$ as internal standards. Chemical shifts of ${ }^{13} \mathrm{C}$ NMR spectra were reported in ppm from the central peak of $\mathrm{CDCl}_{3}(77.16 \mathrm{ppm})$ on the $\delta$ scale. Chemical shifts of ${ }^{19} \mathrm{~F}$ NMR are reported as parts per million in $\delta$ scale using benzotrifluoride $(-63.72 \mathrm{ppm})$ as internal standards. Data are represented as follows: chemical shift, integration, multiplicity $(\mathrm{s}=$ singlet, $\mathrm{d}=$ doublet, $\mathrm{t}=$ triplet, $\mathrm{q}=$ quartet, $\mathrm{qn}=$ quintuplet, $\mathrm{sx}=$ sextet, $\mathrm{sp}=$ septuplet, $\mathrm{m}=$ multiplet, $\mathrm{br}=$ broad), and coupling constant $(J, \mathrm{~Hz})$. High resolution mass spectra (HRMS) were obtained on a Finnigan MAT 95XL GC Mass Spectrometer or a Thermo Scientific Q Exactive Focus Mass Spectrometer or Bruker 9.4T FTICR Mass Spectrometer. The control experiment results were obtained on a Shimadzu GCMSQP2010 SE GC MS Spectrometer. 


\section{Experimental Procedures:}

\section{General procedure (cf. Scheme 2 and 3):}

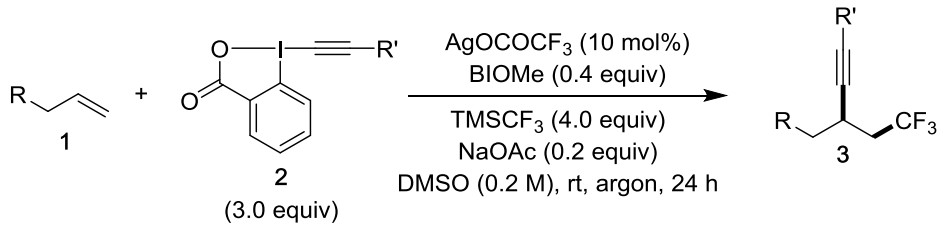

To a glass tube equipped with a magnetic stir bar were added alkene $\mathbf{1}(0.2 \mathrm{mmol}), \mathrm{NaOAc}(0.04$ $\mathrm{mmol}), \mathrm{AgOCOCF}_{3}(0.02 \mathrm{mmol}), \mathrm{BIOMe}(0.08 \mathrm{mmol})$ and $2(0.6 \mathrm{mmol})$. The tube was sealed with a septum, evacuated and refilled with argon three times. Anhydrous DMSO (1.0 mL) was added via a syringe followed by adding $\mathrm{Me}_{3} \mathrm{SiCF}_{3}$ dropwise $(120 \mu \mathrm{L}, 0.8 \mathrm{mmol})$. The tube was sealed and stirred at room temperature for $24 \mathrm{~h}$. The color slowly changed from dark grey to light yellow. The reaction mixture was quenched with aq. sat. $\mathrm{NH}_{4} \mathrm{Cl}$, extracted with ethyl acetate three times. The organic layers were combined, washed with aq. sat. $\mathrm{NaHCO}_{3}$ then brine, dried over anhydrous $\mathrm{Na}_{2} \mathrm{SO}_{4}$, filtered and concentrated by rotary evaporator. The crude product was purified by flash column chromatography on silica gel to afford the desired product 3 . 
Substrates 1:<smiles>C=CCCCCOC(=O)c1ccccc1</smiles>

1a<smiles>C=CCCCCOC(=O)c1ccccc1OC</smiles>

$1 e$<smiles>C=CCCCCOC(=O)c1ccc(Cl)cc1</smiles><smiles>C=CCCCCCOC(=O)c1cccn1C</smiles><smiles>C=CCCCCOc1ccc(P)cc1</smiles>

$1 q$<smiles>C=CCCCCO[Sb]</smiles><smiles>C=CCCCCOC(=O)c1ccc(C)cc1</smiles>

1b<smiles>C=CCCCCOC(=O)c1ccc(OC)cc1</smiles>

1c<smiles>C=CCCCCOC(=O)c1ccc(C#N)cc1</smiles>

19<smiles>C=CCCCCOC(=O)c1ccc(Br)cc1</smiles>

1j<smiles>C=CCCCCOC(=O)c1cccs1</smiles>

1n

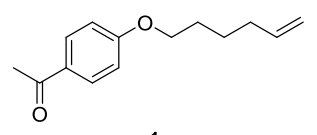

$1 \mathrm{r}$<smiles>C=CCCCCO[AsH3]</smiles><smiles>C=CCCCCOC(=O)c1ccc(I)cc1</smiles>

$1 \mathrm{k}$<smiles>C=CCCCCOC(=O)c1ccccn1</smiles>

10<smiles>C=CCCCCOc1ccc(C=O)cc1</smiles>

$1 \mathrm{~s}$

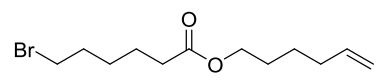<smiles>C=CCCCCCOC(=O)c1cccc(OC)c1</smiles><smiles>C=CCCCCOC(=O)c1ccc(F)cc1</smiles>

$1 \mathrm{~h}$<smiles>C=CCCCCOC(=O)c1ccco1</smiles>

11<smiles>C=CCCCCOc1ccc(I)cc1</smiles>

$1 p$<smiles>C=CCCCCOCc1ccccc1</smiles>

$1 t$<smiles>C=CCCCCCCCCC(=O)OC</smiles><smiles>C=CCCCCCCCCCC(=O)O</smiles>

1 aa<smiles>C=C(C)CCOC(=O)c1ccccc1</smiles>

$1 a b$

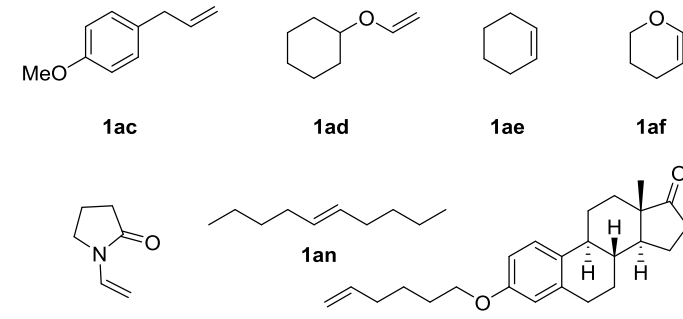

1 ah

1 ai<smiles>C=Cc1ccc(OC)cc1</smiles><smiles>C=CC(=O)Oc1ccc2ccccc2c1</smiles>

1al<smiles>C=CCCCCOc1c(-c2ccccc2)oc2ccccc2c1=O</smiles>

1ak

Alkenes 1a-1w, 1y, 1ab, 1am, 1ai-1ak, were synthesized according to literature procedure. ${ }^{1} \mathbf{1 x}, \mathbf{1 z}$, 1aa, 1ac-1ah, 1al, 1an were commercially available. 
Substrates 2:

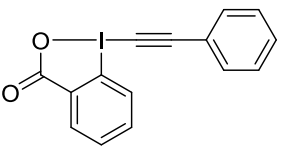

2a<smiles>O=C1OI(C#Cc2ccc(Br)cc2)c2ccccc21</smiles>

$2 e$

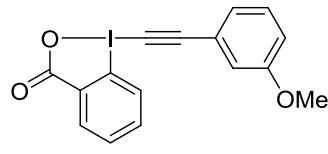

$\mathbf{2 b}$

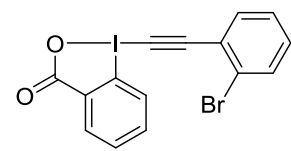

$2 f$

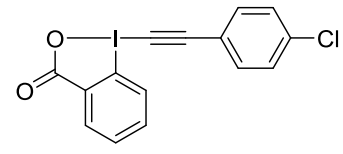

2c

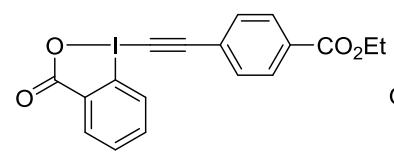

$2 \mathrm{~g}$

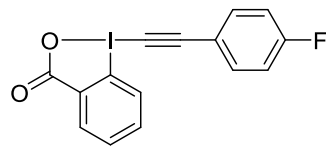

2d

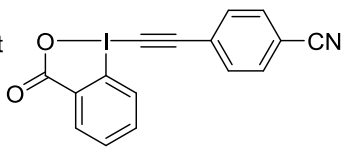

2h

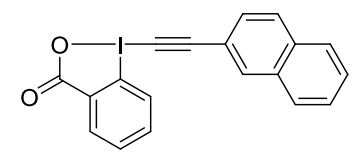

2i

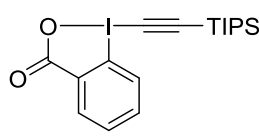

2j<smiles>CCC#Cc1ccccc1C(=O)O</smiles>

2k

EBX reagents $\mathbf{2} \mathbf{a}-\mathbf{2} \mathbf{h}, \mathbf{2} \mathbf{j}-\mathbf{2} \mathbf{k}$ were synthesized according to literature procedure. ${ }^{2}$

\section{Preparation of 1-[naphthylethynyl]-1,2-benziodoxol-3(1H)-one (2i).}<smiles>O=C1OI(O)c2ccccc21</smiles><smiles>CCO[Mg]CC#Cc1ccc2ccccc2c1</smiles><smiles>O=C1OI(C#Cc2ccc3ccccc3c2)c2ccccc21</smiles>

Following a literature procedure, ${ }^{2}$ trimethylsilyltriflate $(1.1 \mathrm{~mL}, 6.0 \mathrm{mmol})$ was added dropwise to a solution of 1-hydroxy-1,2-benziodoxol-3-(1H)-one $(1.43 \mathrm{~g}, 5.45 \mathrm{mmol})$ in $\mathrm{CH}_{2} \mathrm{Cl}_{2}(15 \mathrm{~mL})$ at room temperature. The yellow solution was stirred at $\mathrm{rt}$ for $1.5 \mathrm{~h}$, then a solution of trimethyl(naphthalen-2ylethynyl)silane $(1.34 \mathrm{~g}, 6.0 \mathrm{mmol})$ in $\mathrm{CH}_{2} \mathrm{Cl}_{2}(2.0 \mathrm{~mL})$ was slowly added to the above mixture and the reaction mixture was stirred overnight at room temperature. An aq. saturated solution of $\mathrm{NaHCO}_{3}(100$ $\mathrm{mL}$ ) was added and stirred for extra 30 minutes. The resulting suspension was filtered and washed with $\mathrm{CH}_{2} \mathrm{Cl}_{2}$. The two layers of the mother liquors were separated and the organic layer was washed with aq. sat. $\mathrm{NaHCO}_{3}$ then brine, dried over $\mathrm{MgSO}_{4}$, filtered and evaporated under reduced pressure. The solid obtained was boiled in $\mathrm{CH}_{3} \mathrm{CN}$ for about $5 \mathrm{~min}$. The mixture was cooled down, filtered, washed with $\mathrm{CH}_{3} \mathrm{CN}$ and dried under high vacuum to afford the desired product ( $850 \mathrm{mg}, 2.2 \mathrm{mmol}, 40 \%$ ) as a white solid. ${ }^{1} \mathbf{H}$ NMR $\left(500 \mathrm{MHz}, \mathrm{CDCl}_{3}\right): \delta 8.38(\mathrm{dd}, J=7.0 \mathrm{~Hz}, J=2.0 \mathrm{~Hz}, 1 \mathrm{H}), 8.24(\mathrm{~d}, J=9.0 \mathrm{~Hz}, 1 \mathrm{H})$, $8.10(\mathrm{~s}, 1 \mathrm{H}), 7.84-7.80(\mathrm{~m}, 3 \mathrm{H}), 7.75-7.69(\mathrm{~m}, 2 \mathrm{H}), 7.54-7.50(\mathrm{~m}, 3 \mathrm{H}) \mathrm{ppm} .{ }^{13} \mathrm{C}$ NMR $(126 \mathrm{MHz}$, $\left.\mathrm{CDCl}_{3}\right): \delta 166.7,135.1,134.1,134.0,132.7,132.7,131.8,131.5,128.8,128.3,128.3,128.1,127.4,126.4$, 117.8, 116.4, 107.3, 50.5 ppm. HRMS m/z (ESI): calcd. for $\mathrm{C}_{19} \mathrm{H}_{11} \mathrm{IO}_{2} \mathrm{Na}[\mathrm{M}+\mathrm{Na}]^{+}: 420.0696$; found: 420.9696.

2 (a) Huang, H.; Zhang, G.; Gong, L.; Zhang, S.; Chen, Y. J. Am. Chem. Soc. 2014, 136, 2280. (b) Hari, D. P.; Waser, J. J. Am. Chem. Soc. 2016, 138, 2190. (3) Lu, B.; Wu, J.; Yoshikai, N. J. Am. Chem. Soc. 2014, 136, 11598. 
Scale-up reactions (cf. Scheme 2 , compounds $3 y$ and $3 u)$ :
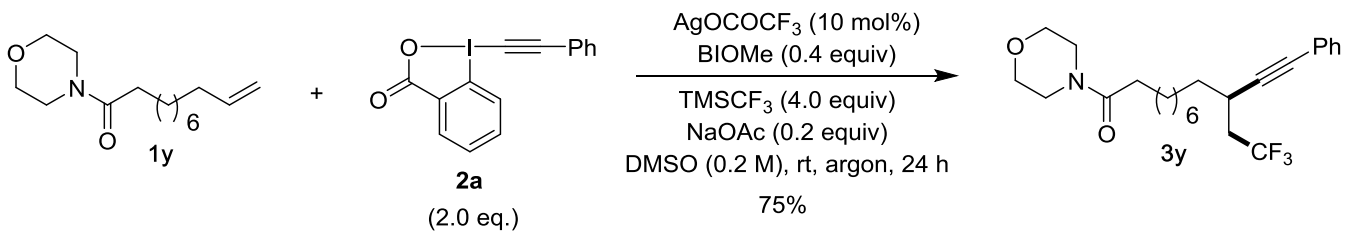

Followed the general procedure: to a $25 \mathrm{~mL}$ round-bottomed flask equipped with a magnetic stir bar were added alkene $1 \mathbf{y}(253.0 \mathrm{mg}, 1.0 \mathrm{mmol}), \mathrm{NaOAc}(16.4 \mathrm{mg}, 0.2 \mathrm{mmol}), \mathrm{AgOCOCF}_{3}(22.2 \mathrm{mg}, 0.1$ mmol), BIOMe (111.2 mg, $0.4 \mathrm{mmol})$ and $2 \mathrm{a}(696.0 \mathrm{mg}, 2.0 \mathrm{mmol})$. The tube was sealed with a septum, evacuated and refilled with argon three times. Anhydrous DMSO (5.0 mL) was added via a syringe followed by adding $\mathrm{Me}_{3} \mathrm{SiCF}_{3}$ dropwise $(0.6 \mathrm{~mL}, 4.0 \mathrm{mmol})$. The flask was sealed and stirred at room temperature for $36 \mathrm{~h}$. The reaction mixture was quenched with aq. sat. $\mathrm{NH}_{4} \mathrm{Cl}$, extracted with ethyl acetate three times. The organic layers were combined, washed with aq. sat. $\mathrm{NaHCO}_{3}$ then brine, dried over anhydrous $\mathrm{Na}_{2} \mathrm{SO}_{4}$, filtered and concentrated by rotary evaporator. The product was purified by flash column chromatography on silica gel (hexane/acetone) and obtained $\mathbf{3 y}$ as a colorless oil (0.75 mmol, $318 \mathrm{mg}, 75 \%$ ), $\mathrm{R}_{\mathrm{f}}=0.30$ (hexane $:$ acetone $=3: 1$ ).

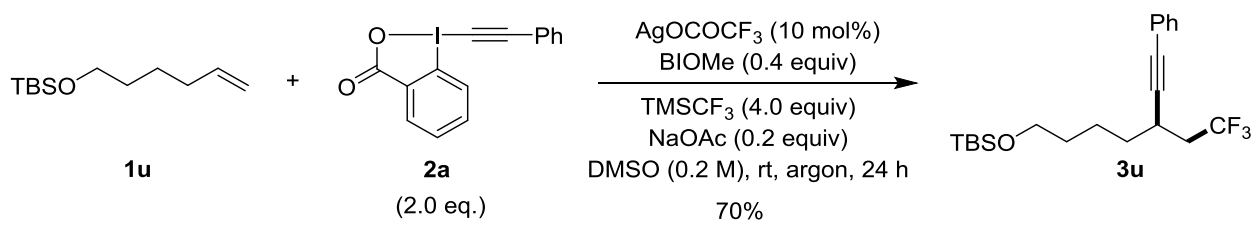

Followed the general procedure: to a $25 \mathrm{~mL}$ round-bottome flask equipped with a magnetic stir bar were added alkene $1 \mathbf{u}(642.0 \mathrm{mg}, 3.0 \mathrm{mmol}), \mathrm{NaOAc}(49.2 \mathrm{mg}, 0.6 \mathrm{mmol}), \mathrm{AgOCOCF}_{3}(66.6 \mathrm{mg}, 0.3$ mmol), BIOMe (333.6 mg, $1.2 \mathrm{mmol})$ and $\mathbf{2 a}(2.1 \mathrm{mg}, 6.0 \mathrm{mmol})$. The flask was sealed with a septum, evacuated and refilled with argon three times. Anhydrous DMSO $(15 \mathrm{~mL})$ was added via a syringe followed by adding $\mathrm{Me}_{3} \mathrm{SiCF}_{3}$ dropwise $(1.8 \mathrm{~mL}, 12.0 \mathrm{mmol})$. Then the tube was sealed and stirred at room temperature for $48 \mathrm{~h}$. The reaction mixture was quenched with aq. sat. $\mathrm{NH}_{4} \mathrm{Cl}$, extracted with ethyl acetate three times. The organic layers were combined, washed with aq. sat. $\mathrm{NaHCO}_{3}$ then brine, dried over anhydrous $\mathrm{Na}_{2} \mathrm{SO}_{4}$, filtered and concentrated by rotary evaporator. The product was purified by flash column chromatography on silica gel (hexane/EtOAc) and obtained $\mathbf{3 u}$ as a colorless oil (2.1 mmol, $807 \mathrm{mg}, 70 \%$ ), $\mathrm{R}_{\mathrm{f}}=0.30$ (hexane).

\section{Further derivatization (cf. Scheme 4):}

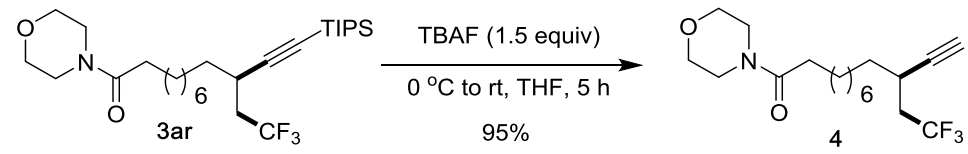

To a solution of $\mathbf{3 a r}(100.6 \mathrm{mg}, 0.2 \mathrm{mmol})$ in THF $(2.0 \mathrm{~mL})$ was added TBAF dropwise $(0.3 \mathrm{~mL}$, $0.3 \mathrm{mmol}, 1.0 \mathrm{M}$ in THF) at $0{ }^{\circ} \mathrm{C}$, then it was warmed to room temperature and stirred for 5 hours. The reaction mixture was quenched with water, extracted with ethyl acetate three times, the organic phases were combined and washed with brine, dried over anhydrous $\mathrm{MgSO}_{4}$, filtered and concentrated by rotary evaporator. The residue was purified by flash column chromatography on silica gel (hexane/EtOAc) and obtained 4 as a colorless oil $(0.19 \mathrm{mmol}, 66.0 \mathrm{mg}, 95 \%), \mathrm{R}_{\mathrm{f}}=0.20$ (hexane : EtOAc $\left.=1.5: 1\right) .{ }^{1} \mathbf{H}$ NMR 
(400 MHz, $\left.\mathrm{CDCl}_{3}\right): \delta 3.61(\mathrm{~m}, 4 \mathrm{H}), 3.57(\mathrm{~m}, 2 \mathrm{H}), 3.42(\mathrm{~m}, 2 \mathrm{H}), 2.67-2.60(\mathrm{~m}, 1 \mathrm{H}), 2.38-2.23(\mathrm{~m}, 1 \mathrm{H})$, $2.26(\mathrm{t}, J=7.6 \mathrm{~Hz}, 2 \mathrm{H}), 2.21-2.11(\mathrm{~m}, 1 \mathrm{H}), 2.09(\mathrm{t}, J=2.0 \mathrm{~Hz}, 1 \mathrm{H}), 1.61-1.54(\mathrm{~m}, 2 \mathrm{H}), 1.51-1.42(\mathrm{~m}$, $3 \mathrm{H}), 1.39-1.34(\mathrm{~m}, 1 \mathrm{H}), 1.32-1.23(\mathrm{~m}, 8 \mathrm{H}) \mathrm{ppm} .{ }^{13} \mathbf{C} \mathbf{N M R}\left(101 \mathrm{MHz}, \mathrm{CDCl}_{3}\right): \delta 171.9,126.2\left(\mathrm{q}, J_{C-F}=\right.$ $278.4 \mathrm{~Hz}$ ), 84.7, 70.4, 67.0, 66.7, 46.1, 41.9, 38.9 (q, $\left.J_{C-F}=28.1 \mathrm{~Hz}\right), 34.5,33.1,29.4,29.3,29.3,29.1$, 26.7, $25.6\left(\mathrm{q}, J_{C-F}=3.0 \mathrm{~Hz}\right), 25.2 \mathrm{ppm} .{ }^{19} \mathbf{F}$ NMR (470 MHz, $\left.\mathrm{CDCl}_{3}\right): \delta-65.27(\mathrm{t}, J=10.6 \mathrm{~Hz}, 3 \mathrm{~F})$. HRMS m/z (APCI): calcd. for $\mathrm{C}_{18} \mathrm{H}_{29} \mathrm{~F}_{3} \mathrm{NO}_{2}[\mathrm{M}+\mathrm{H}]^{+}$: 348.2145 ; found: 348.2143 .

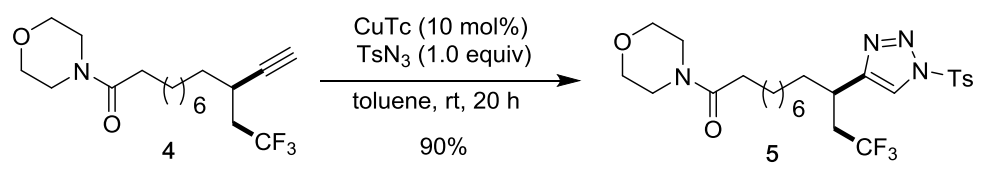

Followed a literature procedure ${ }^{3}$ : under argon, to a solution of $4(66.0 \mathrm{mg}, 0.19 \mathrm{mmol})$ and $\mathrm{CuTc}$ $(3.8 \mathrm{mg}, 0.019 \mathrm{mmol})$ in dry and deoxygenated toluene $(3.0 \mathrm{~mL})$ was added a solution of TsN $\mathrm{N}_{3}(39.5 \mathrm{mg}$, $0.2 \mathrm{mmol})$ in toluene $(1.0 \mathrm{~mL})$, then the reaction was stirred at room temperature under argon for $12 \mathrm{~h}$. The solvent was removed and the residue was directly purified by flash column chromatography on silica gel (hexane/EtOAc) and obtained 5 as a colorless oil $(0.17 \mathrm{mmol}, 92.5 \mathrm{mg}, 90 \%), \mathrm{R}_{\mathrm{f}}=0.30$ (hexane : aceone $=1.5: 1) .{ }^{1} \mathbf{H}$ NMR $\left(500 \mathrm{MHz}, \mathrm{CDCl}_{3}\right): \delta 7.90(\mathrm{~d}, J=8.0 \mathrm{~Hz}, 2 \mathrm{H}), 7.89(\mathrm{~s}, 1 \mathrm{H}), 7.33(\mathrm{~d}, J=8.0$ $\mathrm{Hz}, 2 \mathrm{H}), 3.61(\mathrm{~m}, 4 \mathrm{H}), 3.56(\mathrm{t}, J=4.5 \mathrm{~Hz}, 2 \mathrm{H}), 3.42$ (t, $J=4.8 \mathrm{~Hz}, 2 \mathrm{H}), 3.13-3.07(\mathrm{~m}, 1 \mathrm{H}), 2.60-2.49$ (m, 1H), 2.43-2.34 (m, 1H), $2.39(\mathrm{~s}, 3 \mathrm{H}), 2.25(\mathrm{t}, J=7.8 \mathrm{~Hz}, 2 \mathrm{H}), 1.71-1.65(\mathrm{~m}, 2 \mathrm{H}), 1.57-1.52(\mathrm{~m}, 2 \mathrm{H})$, 1.27-1.02 (m, 10H) ppm. ${ }^{13} \mathbf{C}$ NMR (126 MHz, $\left.\mathrm{CDCl}_{3}\right): \delta 171.8,148.9,147.3,133.1,130.4,128.5,126.4$ $\left(\mathrm{q}, J_{C-F}=278.0 \mathrm{~Hz}\right), 120.9,66.9,66.7,46.0,41.8,38.4\left(\mathrm{q}, J_{C-F}=27.7 \mathrm{~Hz}\right), 34.6,33.0,30.8,29.3,29.2$, 29.1, 29.0, 26.6, 25.1, 21.8 ppm. ${ }^{19}$ F NMR (470 MHz, $\left.\mathrm{CDCl}_{3}\right): \delta-64.96$ (t, $\left.J=10.8 \mathrm{~Hz}, 3 \mathrm{~F}\right)$. HRMS m/z (APCI): calcd. for $\mathrm{C}_{25} \mathrm{H}_{36} \mathrm{~F}_{3} \mathrm{~N}_{4} \mathrm{O}_{4} \mathrm{~S}[\mathrm{M}+\mathrm{H}]^{+}$: 545.2404; found: 545.2403.
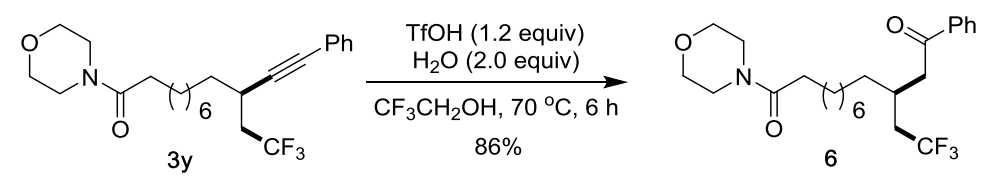

Followed a modified literature procedure: ${ }^{4}$ : to a solution of $3 \mathbf{y}(84.6 \mathrm{mg}, 0.2 \mathrm{mmol})$ in $\mathrm{CF}_{3} \mathrm{CH}_{2} \mathrm{OH}$ $(1.0 \mathrm{~mL})$ in a Schlenk tube equipped with a stirred bar was added $\mathrm{H}_{2} \mathrm{O}(10 \mu \mathrm{L}, 0.4 \mathrm{mmol})$ and $\mathrm{CF}_{3} \mathrm{SO}_{3} \mathrm{H}$ $(21 \mu \mathrm{L}, 0.24 \mathrm{mmol})$. The tube was sealed and placed into a preheated $70{ }^{\circ} \mathrm{C}$ oil bath stirred for 6 hours. Cooled down to room temperature and the solvent was removed, the residue was directly purified by flash column chromatography on silica gel (hexane/EtOAc) and obtained 6 as a colorless oil $(0.17 \mathrm{mmol}$, $75.9 \mathrm{mg}, 86 \%), \mathrm{R}_{\mathrm{f}}=0.30$ (hexane : aceone $\left.=2: 1\right) .{ }^{1} \mathbf{H} \mathbf{~ N M R}\left(400 \mathrm{MHz}, \mathrm{CDCl}_{3}\right): \delta 7.91(\mathrm{~d}, J=7.6 \mathrm{~Hz}$, 2H), $7.54(\mathrm{t}, J=7.4 \mathrm{~Hz}, 1 \mathrm{H}), 7.43(\mathrm{t}, J=7.6 \mathrm{~Hz}, 2 \mathrm{H}), 3.62(\mathrm{~m}, 4 \mathrm{H}), 3.58(\mathrm{~m}, 2 \mathrm{H}), 3.42(\mathrm{t}, J=4.6 \mathrm{~Hz}$, $2 \mathrm{H}), 3.02(\mathrm{~m}, 2 \mathrm{H}), 2.49-2.40(\mathrm{~m}, 1 \mathrm{H}), 2.27(\mathrm{t}, J=7.8 \mathrm{~Hz}, 2 \mathrm{H}), 2.23-2.13(\mathrm{~m}, 2 \mathrm{H}), 1.62-1.54(\mathrm{~m}, 2 \mathrm{H})$, $1.46-1.40(\mathrm{~m}, 2 \mathrm{H}), 1.34-1.21(\mathrm{~m}, 10 \mathrm{H}) \mathrm{ppm} .{ }^{13} \mathbf{C}$ NMR (101 MHz, $\left.\mathrm{CDCl}_{3}\right): \delta 198.8,171.9,137.0,133.2$, $128.7,128.0,127.3\left(\mathrm{q}, J_{C-F}=278.4 \mathrm{~Hz}\right), 67.0,66.7,46.1,42.1,41.9,37.1$ (q, $\left.J_{C-F}=27.3 \mathrm{~Hz}\right), 33.9,33.1$, 29.5, 29.4, 29.3, 29.3, 28.8, 26.5, 25.2 ppm. ${ }^{19}$ F NMR (470 MHz, $\left.\mathrm{CDCl}_{3}\right): \delta-64.96(\mathrm{t}, J=11.5 \mathrm{~Hz}, 3 \mathrm{~F})$. HRMS m/z (APCI): calcd. for $\mathrm{C}_{24} \mathrm{H}_{35} \mathrm{~F}_{3} \mathrm{NO}_{3}[\mathrm{M}+\mathrm{H}]^{+}:$:42.2564; found: 442.2562 .

3 (1) Miura, T.; Funakoshi, Y.; Murakami, M. J. Am. Chem. Soc. 2014, 136, 2272. (2) Fu, L.; Zhou, S.; Wan, X.; Chen, P.; Liu, G. J. Am. Chem. Soc. 2018, 140, 10965.

4 Liu, W.; Wang, H.; Li, C.-J. Org. Lett. 2016, 18, 2184. 


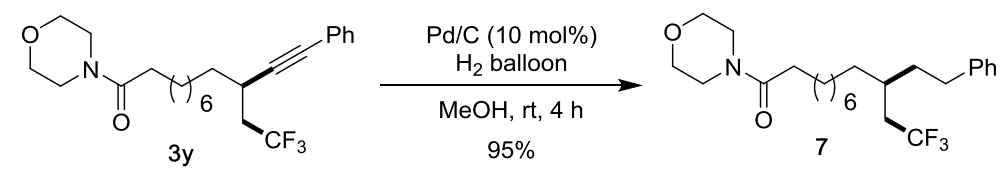

To a $10 \mathrm{~mL}$ round-bottomed flask charged with a stirred bar was added $\mathbf{3 y}$ (84.6 $\mathrm{mg}, 0.2 \mathrm{mmol}$ ), $\mathrm{Pd} / \mathrm{C}(21.1 \mathrm{mg}, 0.02 \mathrm{mmol}, 10 \%)$ and $\mathrm{MeOH}(4.0 \mathrm{~mL})$, The mixture was carefully evacuated and backfilled with hydrogen three times then stirred at room temperature for 3 hours under a $\mathrm{H}_{2}$ balloon atmosphere. Filtered to remove insoluble materials, the solvent was removed under reduced pressure and the residue was directly purified by flash column chromatography on silica gel (hexane/EtOAc) and obtained 7 as a colorless oil $(0.19 \mathrm{mmol}, 81.1 \mathrm{mg}, 95 \%), \mathrm{R}_{\mathrm{f}}=0.4$ (hexane : aceone $\left.=2: 1\right) .{ }^{1} \mathbf{H}$ NMR $\left(400 \mathrm{MHz}, \mathrm{CDCl}_{3}\right): \delta 7.30(\mathrm{t}, J=7.6 \mathrm{~Hz}, 2 \mathrm{H}), 7.22-7.19(\mathrm{~m}, 3 \mathrm{H}), 3.68(\mathrm{t}, J=4.4 \mathrm{~Hz}, 4 \mathrm{H}), 3.64(\mathrm{t}, J=$ $4.4 \mathrm{~Hz}, 2 \mathrm{H}), 3.47(\mathrm{t}, J=4.6 \mathrm{~Hz}, 2 \mathrm{H}), 2.62(\mathrm{t}, J=8.2 \mathrm{~Hz}, 2 \mathrm{H}), 2.33(\mathrm{t}, J=7.6 \mathrm{~Hz}, 2 \mathrm{H}), 2.16-2.06(\mathrm{~m}, 2 \mathrm{H})$, 1.85-1.79 (m, 1H), 1.73-1.62 (m, 4H), 1.49-1.41 (m, 2H), 1.38-1.27 (m, 10H) ppm. ${ }^{13}$ C NMR (101 MHz, $\left.\mathrm{CDCl}_{3}\right): \delta 171.2,142.0,128.4,128.3,127.4\left(\mathrm{q}, J_{C-F}=278.5 \mathrm{~Hz}\right), 123.3,66.9,66.7,46.0,41.9,37.3(\mathrm{q}$, $\left.J_{C-F}=27.0 \mathrm{~Hz}\right), 35.3,33.3,33.1,32.5,32.0,29.7,29.4,29.4,29.4,26.0,25.2$ ppm. ${ }^{19} \mathbf{F}$ NMR $(470 \mathrm{MHz}$, $\left.\mathrm{CDCl}_{3}\right): \delta-64.28(\mathrm{t}, J=11.5 \mathrm{~Hz}, 3 \mathrm{~F})$. HRMS m/z (APCI): calcd. for $\mathrm{C}_{24} \mathrm{H}_{37} \mathrm{~F}_{3} \mathrm{NO}_{2}[\mathrm{M}+\mathrm{H}]^{+}: 428.2771$; found: 428.2769 .

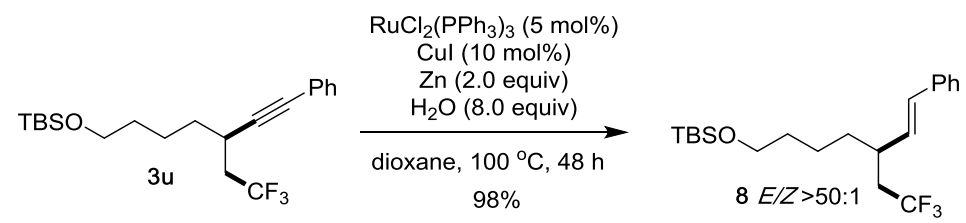

Followed a literature procedure ${ }^{5}$ : a $10 \mathrm{~mL}$ Schlenk tube equipped with a stirred bar was charged with 3u (115.2 mg, $0.3 \mathrm{mmpl})$, zinc (39 mg, $0.6 \mathrm{mmol}), \mathrm{CuI}(5,7 \mathrm{mg}, 0.03 \mathrm{mmol})$ and $\mathrm{RuCl}_{2}\left(\mathrm{PPh}_{3}\right)_{3}(14.4$ $\mathrm{mg}, 0.015 \mathrm{mmol})$, subsequently dioxane $(1.0 \mathrm{~mL})$ and $\mathrm{H}_{2} \mathrm{O}(44 \mu \mathrm{L}, 2.4 \mathrm{mmol})$ were added. The mixture was evacuated and backfilled with argon under $-78{ }^{\circ} \mathrm{C}$ three times, then warmed to room temperature. The tube was sealed and placed into a preheated $100{ }^{\circ} \mathrm{C}$ oil bath stirred for $36 \mathrm{~h}$. Cooled down to room temperature, the solvent was removed and the residue was directly purified by flash column chromatography on silica gel (hexane/EtOAc) and obtained $\mathbf{8}$ as a colorless oil (0.29 mmol, $113.5 \mathrm{mg}$, $98 \%, \mathrm{E} / \mathrm{Z}>50: 1), \mathrm{R}_{\mathrm{f}}=0.2$ (hexane). ${ }^{1} \mathbf{H}$ NMR (400 MHz, $\left.\mathrm{CDCl}_{3}\right): \delta 7.39(\mathrm{~d}, J=7.2 \mathrm{~Hz}, 2 \mathrm{H}), 7.34(\mathrm{t}$, $J=7.6 \mathrm{~Hz}, 2 \mathrm{H}), 7.26(\mathrm{t}, J=7.4 \mathrm{~Hz}, 1 \mathrm{H}), 6.46(\mathrm{~d}, J=15.6 \mathrm{~Hz}, 1 \mathrm{H}), 6.00(\mathrm{dd}, J=15.6 \mathrm{~Hz}, J=9.2 \mathrm{~Hz}$, $1 \mathrm{H}), 3.64(\mathrm{t}, J=6.4 \mathrm{~Hz}, 2 \mathrm{H}), 2.64-2.56(\mathrm{~m}, 1 \mathrm{H}), 2.31-2.18(\mathrm{~m}, 2 \mathrm{H}), 1.64-1.36(\mathrm{~m}, 6 \mathrm{H}), 0.93(\mathrm{~s}, 9 \mathrm{H}), 0.08$ $(\mathrm{s}, 6 \mathrm{H}) \mathrm{ppm} .{ }^{13} \mathbf{C}$ NMR $\left(101 \mathrm{MHz}, \mathrm{CDCl}_{3}\right): \delta 137.3,132.1,131.1,128.6,127.4,126.9$ (q, $J_{C-F}=278.6$ $\mathrm{Hz}), 126.3,63.1,39.4$ (q, $\left.J_{C-F}=26.9 \mathrm{~Hz}\right), 37.7,35.0,32.7,26.1,23.4,18.5,-5.2 \mathrm{ppm} .{ }^{19} \mathbf{F}$ NMR $(470$ $\mathrm{MHz}_{\mathrm{CDCl}}$ ): $\delta-64.11(\mathrm{t}, J=11.0 \mathrm{~Hz}, 3 \mathrm{~F})$. HRMS m/z (APCI): calcd. for $\mathrm{C}_{21} \mathrm{H}_{34} \mathrm{~F}_{3} \mathrm{OSi}[\mathrm{M}+\mathrm{H}]^{+}$: 387.2326; found: 387.2326 .
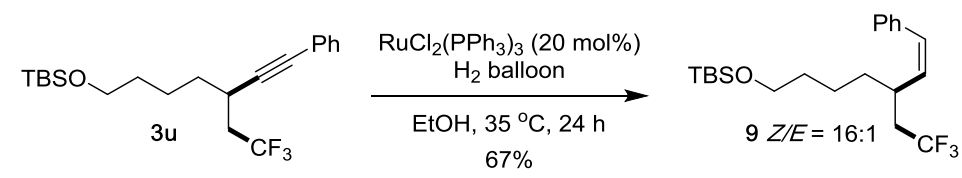

Followed a modified literature procedure:6: to a $10 \mathrm{~mL}$ round-bottomed flask charged with a stirred

5 Schabel, T.; Belger, C.; Plietker, B. Org. Lett. 2013, 15, 2858.

6 Belger, C.; Neisius, N. M.; Plietker, B. Chem. Eur. J. 2010, 16, 12214-12220. 
bar was added $3 \mathbf{u}(76.8 \mathrm{mg}, 0.2 \mathrm{mmol}), \mathrm{RuCl}_{2}\left(\mathrm{PPh}_{3}\right)_{3}(38.4 \mathrm{mg}, 0.04 \mathrm{mmol})$ and $\mathrm{EtOH}(2.0 \mathrm{~mL})$, the mixture then carefully evacuated and backfilled with hydrogen three times then stirred at $35^{\circ} \mathrm{C}$ for 24 hours under a $\mathrm{H}_{2}$ balloon atmosphere. After completion, the solvent was removed under reduced pressure and the residue was directly purified by flash column chromatography on silica gel (hexane/EtOAc) and obtained 9 as a colorless oil $(0.13 \mathrm{mmol}, 51.5 \mathrm{mg}, 67 \%, \mathrm{Z} / \mathrm{E}=16: 1), \mathrm{R}_{\mathrm{f}}=0.4$ (hexane : aceone $=2$ : 1). ${ }^{1} \mathbf{H}$ NMR (500 MHz, $\left.\mathrm{CDCl}_{3}\right): \delta 7.34(\mathrm{~d}, J=8.0 \mathrm{~Hz}, 2 \mathrm{H}), 7.27-7.23(\mathrm{~m}, 3 \mathrm{H}), 6.55(\mathrm{~d}, J=12.0 \mathrm{~Hz}$, $1 \mathrm{H}), 5.42(\mathrm{t}, J=11 \mathrm{~Hz}, 1 \mathrm{H}), 3.55(\mathrm{t}, J=6.0 \mathrm{~Hz}, 2 \mathrm{H}), 3.10-3.01(\mathrm{~m}, 1 \mathrm{H}), 2.25-2.10(\mathrm{~m}, 2 \mathrm{H}), 1.55-1.48$ $(\mathrm{m}, 1 \mathrm{H}), 1.45-1.31(\mathrm{~m}, 4 \mathrm{H}), 1.26-1.18(\mathrm{~m}, 1 \mathrm{H}), 0.90(\mathrm{~s}, 9 \mathrm{H}), 0.04(\mathrm{~s}, 6 \mathrm{H}) \mathrm{ppm} .{ }^{13} \mathbf{C}$ NMR $(126 \mathrm{MHz}$, $\left.\mathrm{CDCl}_{3}\right): \delta 137.3,134.7,130.3,128.5,128.4,126.9\left(\mathrm{q}, J_{C-F}=278.1 \mathrm{~Hz}\right), 126.9,63.0,39.3\left(\mathrm{q}, J_{C-F}=27.0\right.$ $\mathrm{Hz}), 35.3,32.8,32.0,26.1,23.2,18.5,-5.2 \mathrm{ppm} .{ }^{19} \mathbf{F}$ NMR $\left(470 \mathrm{MHz}, \mathrm{CDCl}_{3}\right): \delta-64.04(\mathrm{t}, J=11.0 \mathrm{~Hz}$, 3F). HRMS m/z (APCI): calcd. for $\mathrm{C}_{21} \mathrm{H}_{34} \mathrm{~F}_{3} \mathrm{OSi}[\mathrm{M}+\mathrm{H}]^{+}: 387.2326$; found: 387.2327 . 
Mechanistic studies:

Figure S1. NMR $\left({ }^{1} \mathrm{H}\right.$ and $\left.{ }^{13} \mathrm{C}\right)$ studies of the stability of EBX reagent 2a and BIOMe in the presence of equimolar $\mathrm{AgOCOCF}_{3}$

(a) ${ }^{1} \mathrm{H}$ NMR of 2a and $\mathrm{AgOCOCF}_{3}$ over time
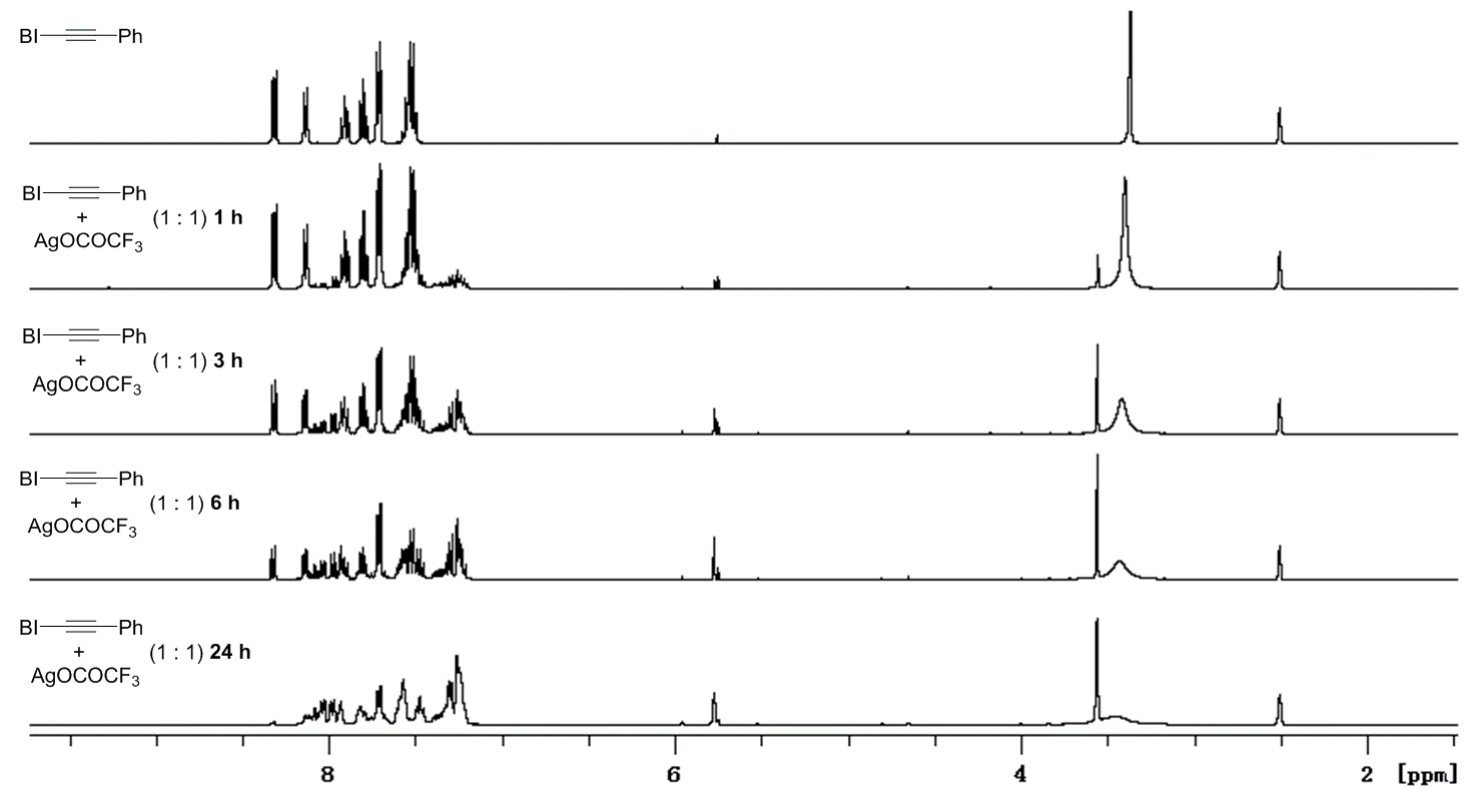

(b) ${ }^{13} \mathrm{C}$ NMR of $2 \mathrm{a}$ and $\mathrm{AgOCOCF}_{3}$ over time
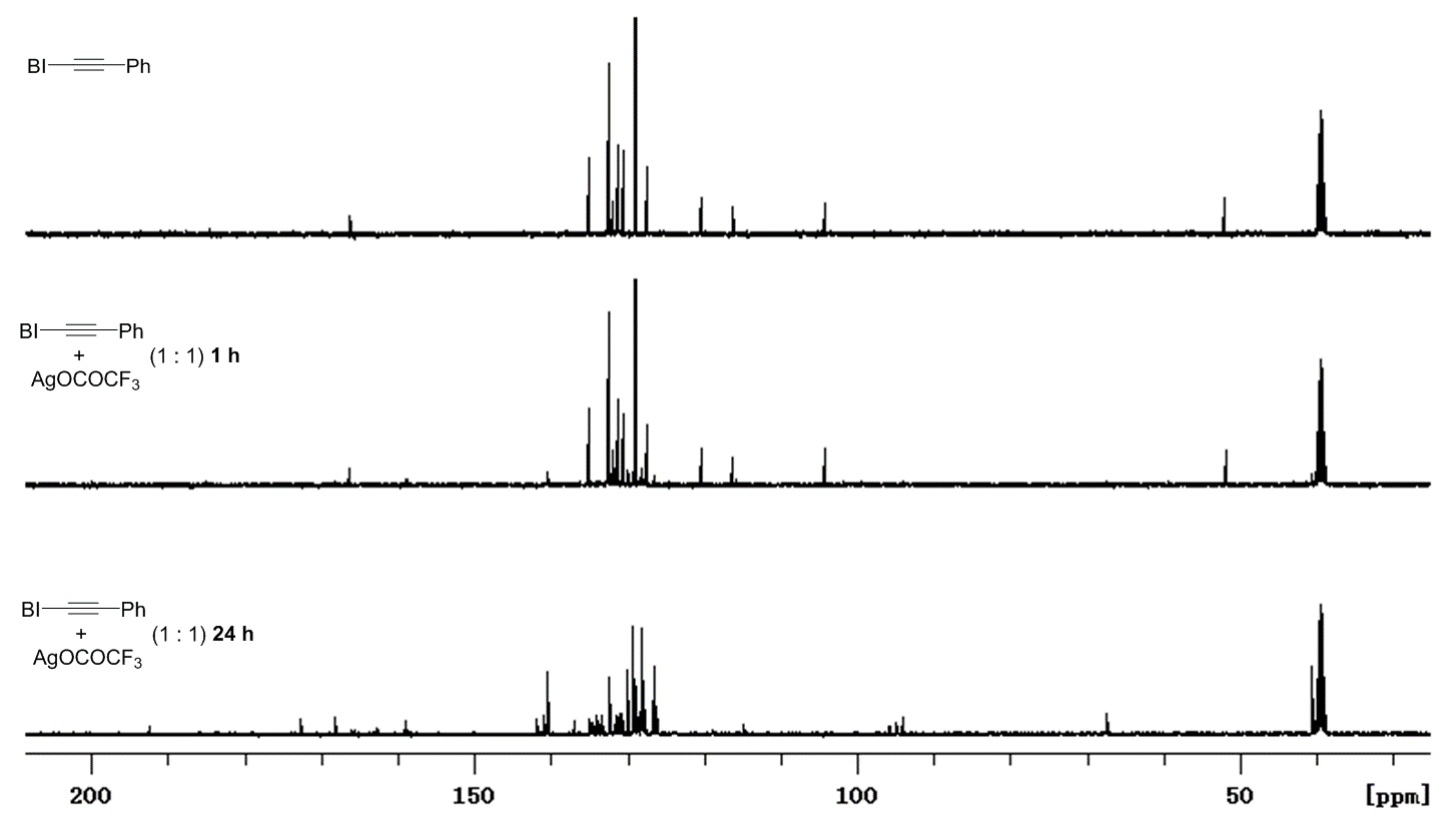
(c) ${ }^{1} \mathrm{H}$ NMR of BIOMe and $\mathrm{AgOCOCF}_{3}$ over time

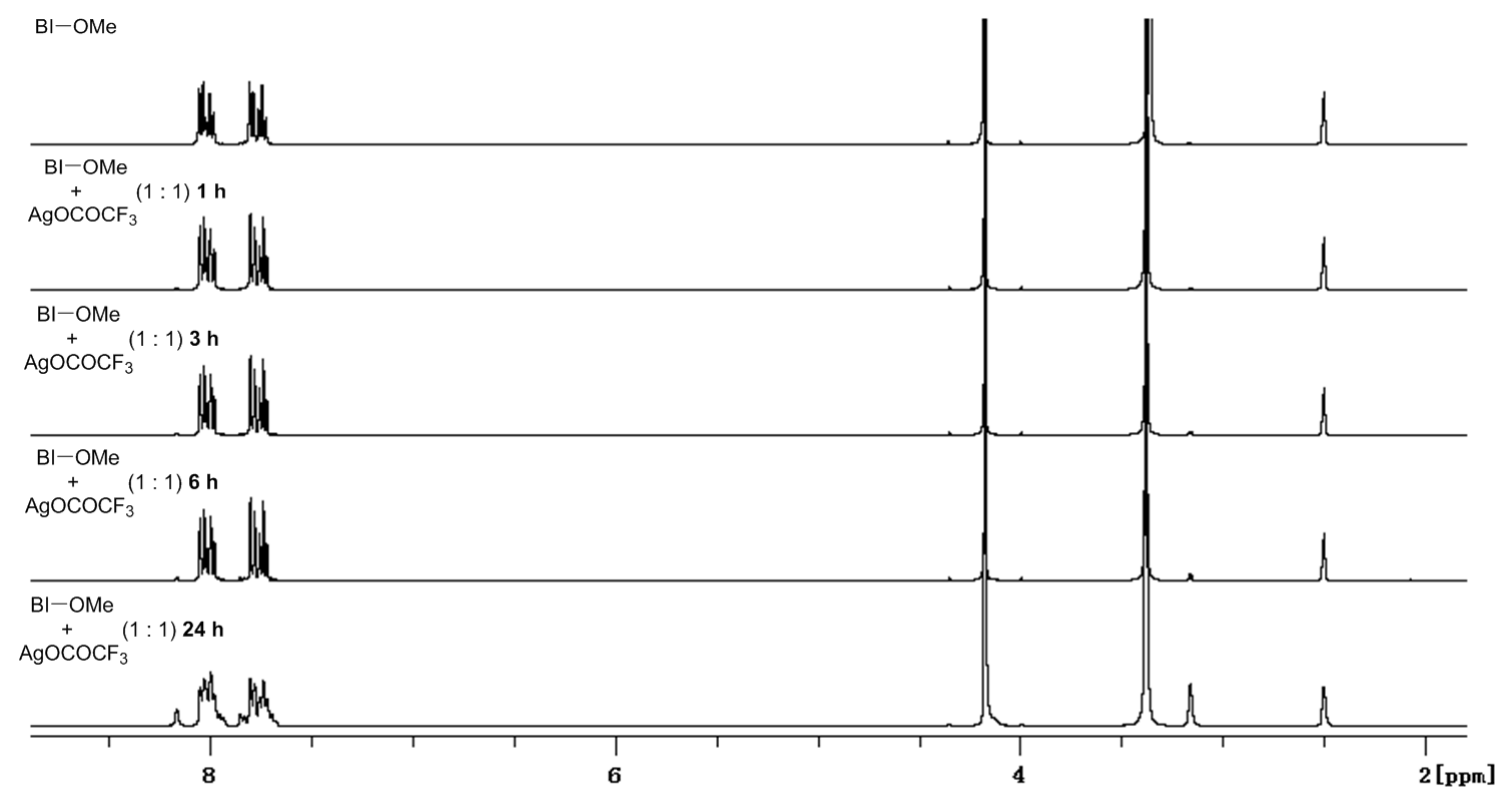

(d) ${ }^{13} \mathrm{C}$ NMR of BIOMe and $\mathrm{AgOCOCF}_{3}$ over time
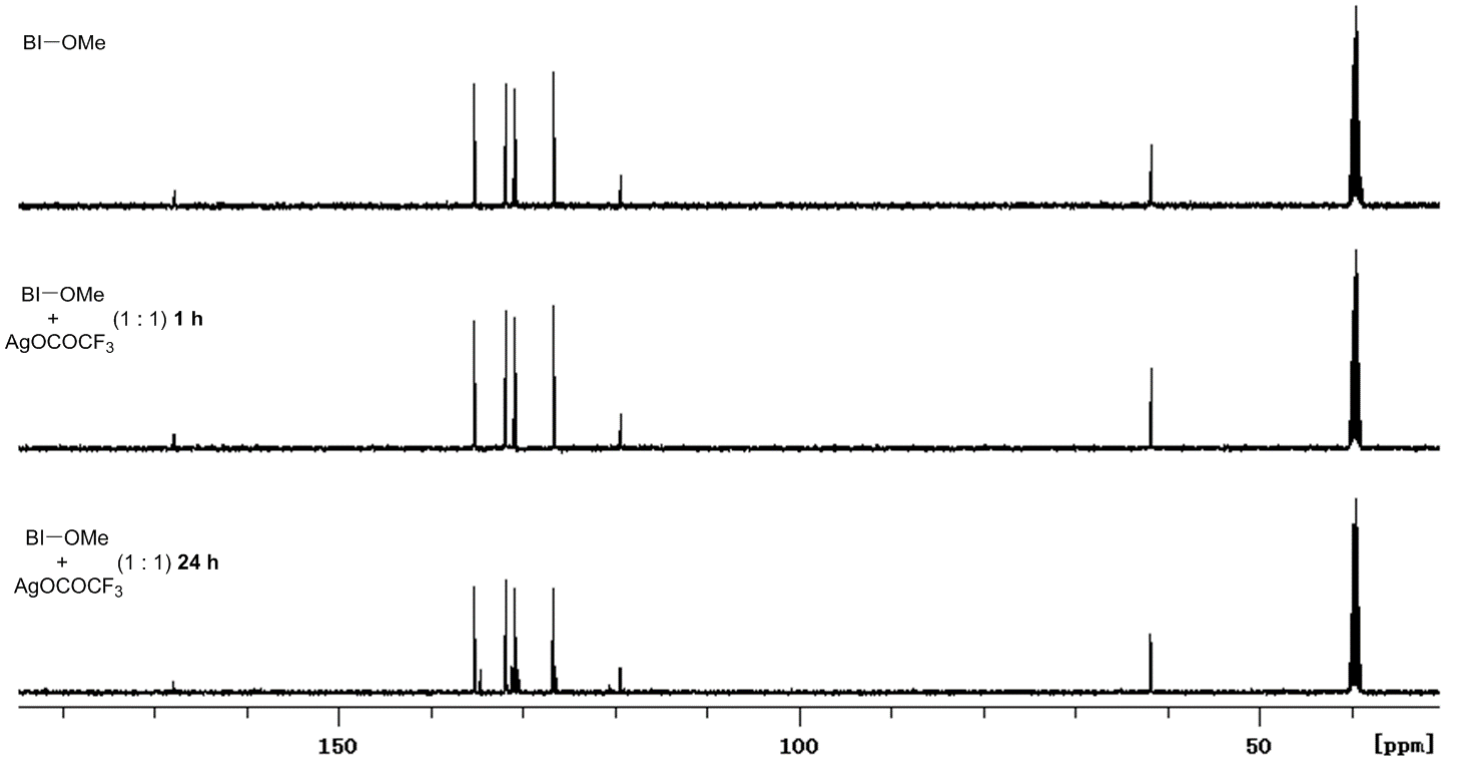
Control experiments with $\mathrm{AgCF}_{3}$ (cf. Eq. 4).

$$
\mathrm{AgF}+\mathrm{TMSCF}_{3} \underset{\text { rt. } 30 \mathrm{mins}}{\stackrel{\mathrm{CH}_{3} \mathrm{CN}}{\longrightarrow}} \mathrm{AgCF}_{3}
$$

Followed a modified literature procedure: ${ }^{7}$ under argon, to an oven-dried glass tube equipped with a stir bar was added $\mathrm{AgF}(63.5 \mathrm{mg}, 0.5 \mathrm{mmol})$, dry and deoxygenated $\mathrm{CH}_{3} \mathrm{CN}(1.5 \mathrm{~mL})$, then $\mathrm{TMSCF}_{3}$ (71 $\mathrm{mg}, 0.5 \mathrm{mmol}$ ) was added dropwise. The mixture was stirred at $\mathrm{rt}$ for $30 \mathrm{~min}$, stopped stirring to make the residue solid subside, a yellow $\mathrm{AgCF}_{3}$ solution in $\mathrm{CH}_{3} \mathrm{CN}$ was formed $(\sim 0.2 \mathrm{M}$ based on the ${ }^{19} \mathrm{~F}$ NMR). The solution was immediately used without further purification. The preparation should be avoided from the light due to the photosensitivity of $\mathrm{AgCF}_{3}$.

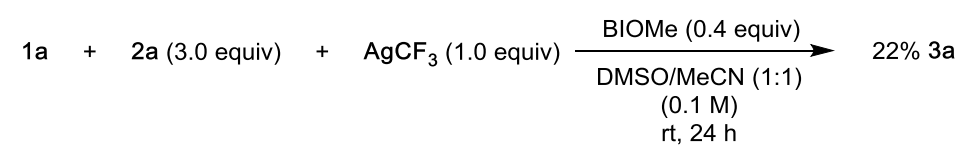

Under argon, to a glass tube equipped with a magnetic stir bar was added alkene 1a (20.4 mg, 0.1 mmol), BI-alkyne 2a (104.4 mg, $0.3 \mathrm{mmol})$, BIOMe (11.1 mg, $0.04 \mathrm{mmol})$. The tube was sealed with a septum, evacuated and refilled with argon three times. Dry DMSO $(0.5 \mathrm{~mL})$ was then added via syringe followed by adding above freshly prepared $\mathrm{AgCF}_{3}(0.5 \mathrm{~mL}, 0.1 \mathrm{mmol})$ dropwise. The mixture was stirred at room temperature under argon for $24 \mathrm{~h}$. The result was analyzed by ${ }^{19} \mathrm{~F}$ NMR using benzotrifluoride as the internal standard.

Radical clock experiments (cf. Eqs. 1 and 2).

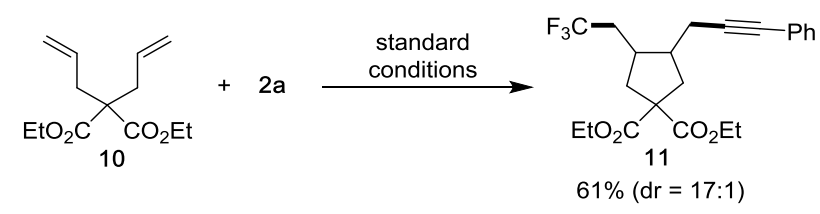

To a glass tube equipped with a magnetic stir bar were added alkene 11 (96.0 mg, $0.4 \mathrm{mmol}), \mathrm{NaOAc}$ (6.6 mg, $0.08 \mathrm{mmol}), \mathrm{AgOCOCF}_{3}(8.8 \mathrm{mg}, 0.04 \mathrm{mmol}), \mathrm{BIOMe}(44.5 \mathrm{mg}, 0.16 \mathrm{mmol})$ and $2 \mathrm{a}(418 \mathrm{mg}$, $1.2 \mathrm{mmol})$. The tube was sealed with a septum, evacuated and refilled with argon three times. Anhydrous DMSO $(2.0 \mathrm{~mL})$ was added via a syringe followed adding $\mathrm{Me}_{3} \mathrm{SiCF}_{3}$ dropwise $(240 \mu \mathrm{L}, 1.6 \mathrm{mmol})$. The tube was sealed and stirred at room temperature for $24 \mathrm{~h}$. The reaction mixture was quenched with aq. sat. $\mathrm{NH}_{4} \mathrm{Cl}$, extracted with ethyl acetate three times. The organic layers were combined, washed with aq. sat. $\mathrm{NaHCO}_{3}$ then brine, dried over anhydrous $\mathrm{Na}_{2} \mathrm{SO}_{4}$, filtered and concentrated by rotary evaporator. The crude mixture was purified by flash column chromatography on silica gel to afford the product $\mathbf{6 b}$ as a colorless oil $(0.24 \mathrm{mmol}, 100 \mathrm{mg}, 61 \%$, $\mathrm{dr}=17: 1), \mathrm{R}_{\mathrm{f}}=0.3$ (hexane : EtOAc $\left.=8: 1\right)$. Major isomer: ${ }^{1} \mathbf{H}$ NMR $\left(400 \mathrm{MHz}, \mathrm{CDCl}_{3}\right): \delta$ 7.40-7.36 (m, 2H), 7.31-7.27 (m, 3H), $4.20(\mathrm{q}, J=7.2 \mathrm{~Hz}$, 2H), $4.18(\mathrm{dq}, J=7.2 \mathrm{~Hz}, J=1.2 \mathrm{~Hz}, 2 \mathrm{H}), 2.61-2.06(\mathrm{~m}, 10 \mathrm{H}), 1.26(\mathrm{t}, J=7.2 \mathrm{~Hz}, 3 \mathrm{H}), 1.23(\mathrm{t}, J=7.2$ $\mathrm{Hz}, 3 \mathrm{H})$ ppm. ${ }^{13} \mathbf{C} \mathbf{N M R}\left(101 \mathrm{MHz}, \mathrm{CDCl}_{3}\right): \delta 172.3,172.3,1316,131.3,128.3,127.9,127.2\left(\mathrm{q}, J_{C-F}=\right.$ $278.3 \mathrm{~Hz}$ ), 123.6, 87.8, 82.2, 61.7, 58.8, 40.9, 38.5, 38.4, 35.9, 33.6 (q, $J_{C-F}=28.2 \mathrm{~Hz}$ ), 20.2, 14.0, 14.0 ppm. ${ }^{19}$ F NMR $\left(470 \mathrm{MHz}, \mathrm{CDCl}_{3}\right)$ : $\delta$ Major isomer: $-65.97(\mathrm{t}, J=10.8 \mathrm{~Hz}, 3 \mathrm{~F})$, Minor isomer: -65.86 (t, $J=10.8 \mathrm{~Hz}, 3 \mathrm{~F})$. HRMS m/z (APCI): calcd. for $\mathrm{C}_{22} \mathrm{H}_{26} \mathrm{~F}_{3} \mathrm{O}_{4}[\mathrm{M}+\mathrm{H}]^{+}: 411.1778$; found: 411.1777 .

7 Zeng, Y.; Zhang, L.; Zhao, Y.; Ni, C.; Zhao, J.; Hu, J. J. Am. Chem. Soc. 2013, 135, 2955. 


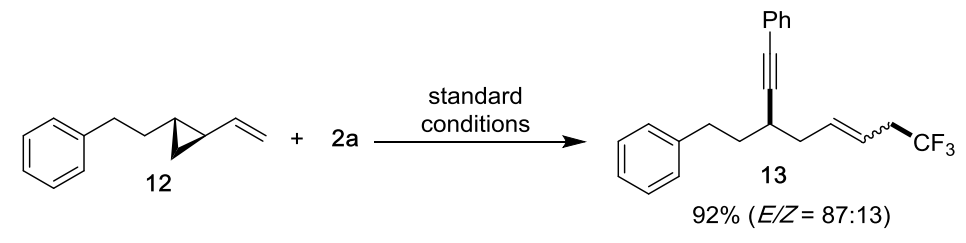

To a glass tube equipped with a magnetic stir bar were added alkene $12(68.8 \mathrm{mg}, 0.4 \mathrm{mmol}),{ }^{1}$ $\mathrm{NaOAc}(6.6 \mathrm{mg}, 0.08 \mathrm{mmol}), \mathrm{AgOCOCF}_{3}(8.8 \mathrm{mg}, 0.04 \mathrm{mmol})$, BIOMe ( $\left.44.5 \mathrm{mg}, 0.16 \mathrm{mmol}\right)$ and $2 \mathrm{a}$ (418 mg, $1.2 \mathrm{mmol})$. The tube was sealed with a septum, evacuated and refilled with argon three times. Anhydrous DMSO $\left(2.0 \mathrm{~mL}\right.$ ) was added via a syringe followed adding $\mathrm{Me}_{3} \mathrm{SiCF}_{3}$ dropwise $(240 \mu \mathrm{L}, 1.6$ $\mathrm{mmol}$ ). The tube was sealed and stirred at room temperature for $24 \mathrm{~h}$. The reaction mixture was quenched with aq. sat. $\mathrm{NH}_{4} \mathrm{Cl}$, extracted with ethyl acetate three times. The organic layers were combined, washed with aq. sat. $\mathrm{NaHCO}_{3}$ then brine, dried over anhydrous $\mathrm{Na}_{2} \mathrm{SO}_{4}$, filtered and concentrated by rotary evaporator. The crude mixture was purified by flash column chromatography on silica gel to afford the product 13 as a colorless oil $(0.37 \mathrm{mmol}, 126 \mathrm{mg}, 92 \%, \mathrm{E} / \mathrm{Z}=87: 13), \mathrm{R}_{\mathrm{f}}=0.50$ (hexane : $\mathrm{EtOAc}=40$ : 1). $\mathbf{E}$ isomer: ${ }^{1} \mathbf{H} \mathbf{N M R}\left(400 \mathrm{MHz}, \mathrm{CDCl}_{3}\right): \delta$ 7.46-7.32 (m, $\left.2 \mathrm{H}\right), 7.34-7.28(\mathrm{~m}, 5 \mathrm{H}), 7.27-7.21(\mathrm{~m}, 3 \mathrm{H})$, 5.89-5.50 (m, 1H), 5.55-5.47 (m, 1H), 2.96-2.86 (m, 1H), 2.85-2.75 (m, 3H), 2.67-2.61 (m, 1H), $2.34(\mathrm{t}$, $J=6.8 \mathrm{~Hz}, 2 \mathrm{H}), 1.84(\mathrm{q}, J=6.8 \mathrm{~Hz}, 2 \mathrm{H}) \mathrm{ppm} .{ }^{13} \mathbf{C} \mathbf{N M R}\left(101 \mathrm{MHz}, \mathrm{CDCl}_{3}\right): \delta 141.9,135.3,131.7$, $128.6,128.5,128.3,127.8,126.1\left(\mathrm{q}, J_{C-F}=277.8 \mathrm{~Hz}\right), 126.0,123.9,120.1,92.1,83.1,37.4\left(\mathrm{q}, J_{C-F}=\right.$ $29.8 \mathrm{~Hz}), 36.4,33.7,32.9,31.7 \mathrm{ppm} .{ }^{19} \mathbf{F}$ NMR $\left(470 \mathrm{MHz}, \mathrm{CDCl}_{3}\right): \delta$ E isomer: -67.77 (t, $J=10.8 \mathrm{~Hz}$, 3F), Z isomer: $-67.31\left(\mathrm{t}, J=10.8 \mathrm{~Hz}, 3 \mathrm{~F}\right.$ ). HRMS m/z (APCI): calcd. for $\mathrm{C}_{22} \mathrm{H}_{22} \mathrm{~F}_{3}[\mathrm{M}+\mathrm{H}]^{+}: 343.1668$; found: 343.1668 . 


\section{Optimization Studies.}

Table S1. Screening of silver(I) salts.

\begin{tabular}{|c|c|c|c|}
\hline Entry & Equiv of $\mathbf{2 a}$ & Silver(I) salt (equiv) & ${ }^{19} \mathrm{~F}$ NMR yield $(\%)$ \\
\hline 1 & 2.0 & none & $<5$ \\
\hline 2 & 2.0 & $\mathrm{AgNO}_{3}(0.2)$ & 23 \\
\hline 3 & 2.0 & $\mathrm{AgNO}_{3}(0.4)$ & 22 \\
\hline 4 & 2.0 & $\mathrm{AgNO}_{3}(1.0)$ & $<5$ \\
\hline 5 & 3.0 & $\mathrm{AgNO}_{3}(0.2)$ & 33 \\
\hline 6 & 3.0 & $\mathrm{AgNO}_{3}(0.1)$ & 31 \\
\hline 7 & 3.0 & $\mathrm{AgClO}_{4}(0.1)$ & 23 \\
\hline 8 & 3.0 & $\mathrm{AgSbF}_{6}(0.1)$ & 31 \\
\hline 9 & 3.0 & $\operatorname{AgF}(0.1)$ & 13 \\
\hline 10 & 3.0 & $\mathrm{Ag}_{2} \mathrm{O}(0.1)$ & 6 \\
\hline 11 & 3.0 & $\operatorname{AgOAc}(0.1)$ & 26 \\
\hline 12 & 3.0 & $\mathrm{Ag}_{2} \mathrm{CO}_{3}(0.1)$ & 6 \\
\hline 13 & 3.0 & $\mathrm{AgPF}_{6}(0.1)$ & 26 \\
\hline 14 & 3.0 & $\operatorname{AgOTf}(0.1)$ & 27 \\
\hline
\end{tabular}

Table S2. Screening of solvents and initiators.

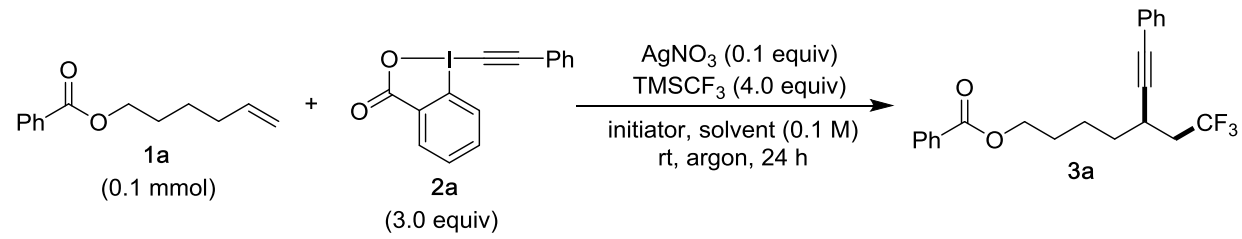

\begin{tabular}{cccc}
\hline Entry & Initiator (equiv) & Solvent & ${ }^{19}$ F NMR yield (\%) \\
\hline 1 & NaOAc $(4.0)$ & DMF & 31 \\
2 & NaOAc $(4.0)$ & THF & 0 \\
3 & NaOAc $(4.0)$ & $\mathrm{CH}_{2} \mathrm{Cl}_{2}$ & 0 \\
4 & $\mathrm{NaOAc}(4.0)$ & Toluene & 0 \\
5 & $\mathrm{NaOAc}(4.0)$ & $\mathrm{CH}_{3} \mathrm{CN}$ & trace \\
6 & $\mathrm{NaOAc}(4.0)$ & $\mathrm{Dioxane}$ & 0 \\
7 & $\mathrm{NaOAc}(4.0)$ & $\mathrm{DMSO}$ & 22 \\
$\mathbf{8}$ & $\mathrm{NaOAc}(\mathbf{0 . 2})$ & $\mathrm{DMF}$ & $\mathbf{4 3}$ \\
9 & $\mathrm{KOAc}(0.2)$ & $\mathrm{DMF}$ & 26 \\
10 & $\mathrm{LiOAc}(0.2)$ & $\mathrm{DMF}$ & 20 \\
11 & $\mathrm{KF}(0.2)$ & $\mathrm{DMF}$ & 20 \\
12 & $\mathrm{TBAF} \cdot 3 \mathrm{H}_{2} \mathrm{O}(0.2)$ & $\mathrm{DMF}$ & 0 \\
13 & $\mathrm{NaOAc}(0.2)+\mathrm{TBAB}(0.2)$ & $\mathrm{DMF}$ & 37 \\
\hline
\end{tabular}


Scheme S1. Screening of alkynylating reagents.

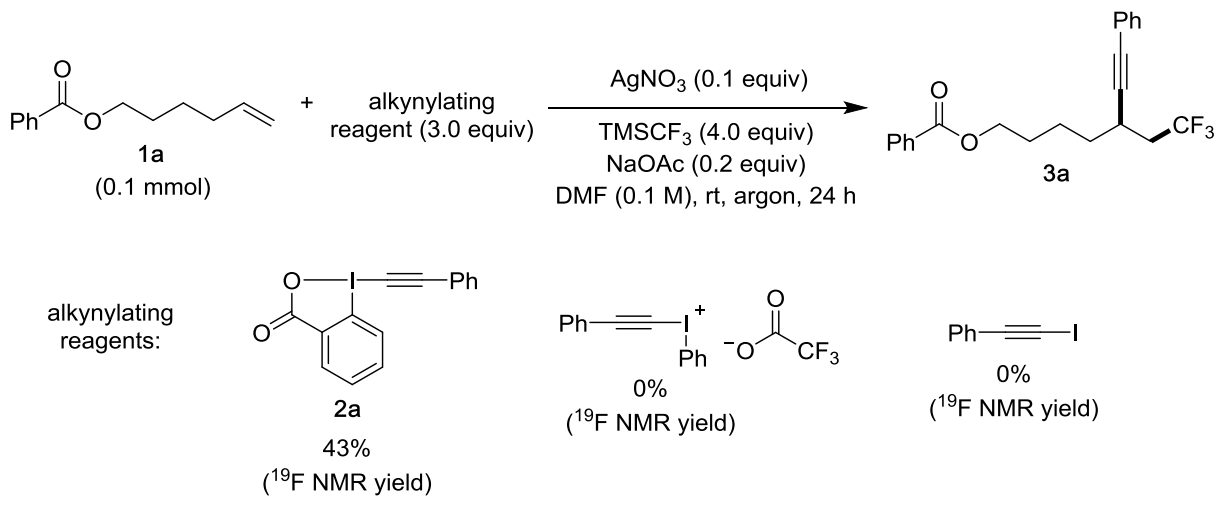

Table S3. Screening of additives.

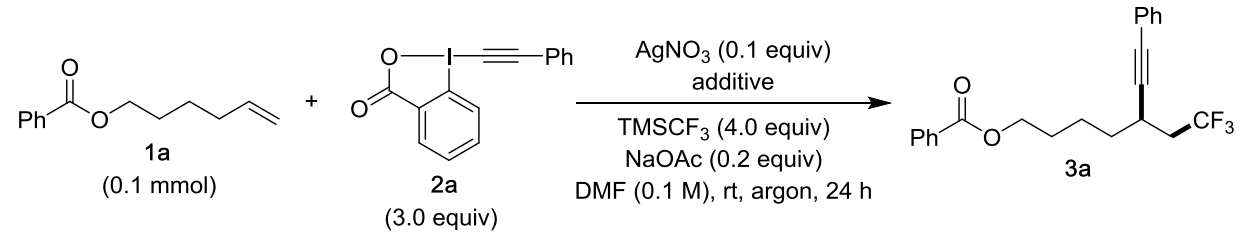

\begin{tabular}{ccc}
\hline Entry & Additive & ${ }^{19} \mathrm{~F}$ NMR yield $(\%)$ \\
\hline 1 & none & 43 \\
2 & molecular sieve 4A $(10 \mathrm{mg})$ & 33 \\
3 & molecular sieve 4A $(20 \mathrm{mg})$ & 31 \\
4 & $\mathrm{BIOH}(0.1$ equiv) & 36 \\
5 & $\mathrm{BIOH}(0.2$ equiv) & 47 \\
$\mathbf{6}$ & $\mathrm{BIOH}(\mathbf{0 . 4}$ equiv) & $\mathbf{5 5}$ \\
7 & $\mathrm{BIOH}(1.0$ equiv) & 47 \\
8 & $\mathrm{PhI}(\mathrm{OAc})_{2}(0.4$ equiv) & 51 \\
\hline
\end{tabular}

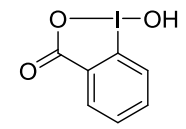

$\mathrm{BIOH}$ 
Table S4. Further screening of silver(I) salts, solvents and concentrations.

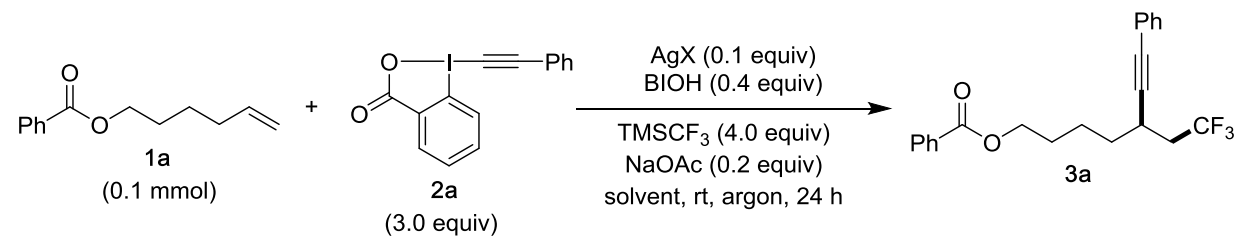

\begin{tabular}{cccc}
\hline Entry & $\operatorname{AgX}$ & Solvent (conc.) & ${ }^{19}$ F NMR yield (\%) \\
\hline 1 & $\operatorname{AgNO}_{3}$ & $\operatorname{DMF}(0.1 \mathrm{M})$ & 55 \\
2 & $\operatorname{AgOCOCF}_{3}$ & $\operatorname{DMF}(0.1 \mathrm{M})$ & 58 \\
3 & $\operatorname{AgNTf}_{2}$ & $\operatorname{DMF}(0.1 \mathrm{M})$ & 59 \\
$4^{\mathrm{a}}$ & $\operatorname{AgOCOCF}_{3}$ & $\operatorname{DMSO}(0.1 \mathrm{M})$ & 60 \\
$5^{\mathrm{a}}$ & $\mathrm{AgOCOCF}_{3}$ & $\operatorname{DMSO}(0.05 \mathrm{M})$ & 42 \\
$6^{\mathrm{a}}$ & $\mathbf{A g O C O C F}_{3}$ & $\operatorname{DMSO}(\mathbf{0 . 2} \mathbf{M})$ & $\mathbf{7 0}$ \\
$7^{\mathrm{a}}$ & $\mathrm{AgOCOCF}_{3}$ & $\operatorname{DMSO}(0.33 \mathrm{M})$ & 65 \\
\hline
\end{tabular}

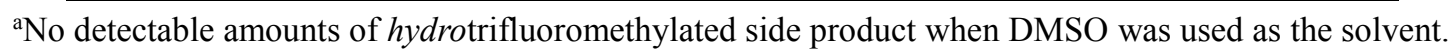


Table S5. Further optimization studies. ${ }^{\mathrm{a}}$

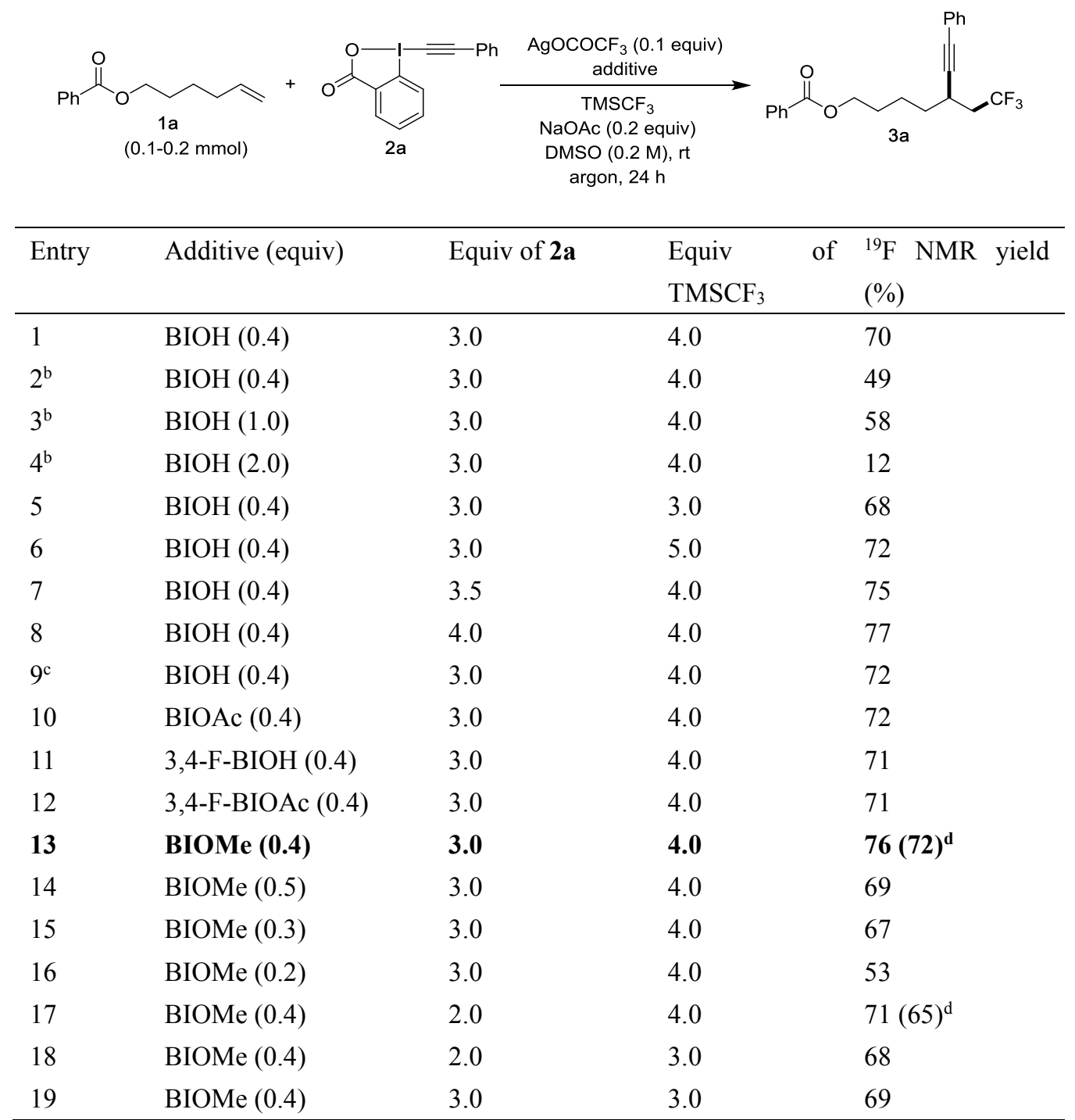

${ }^{\mathrm{a} U n l e s s}$ specified otherwise, $0.1 \mathrm{mmol}$ of substrate 1a was used. ${ }^{\mathrm{b}}$ Without $\mathrm{AgOCOCF}_{3} .{ }^{\mathrm{c}} \mathrm{At} 40{ }^{\circ} \mathrm{C}$. disolated yield using $0.2 \mathrm{mmol}$ of $\mathbf{1 a}$.

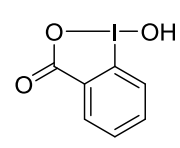

$\mathrm{BIOH}$<smiles>COC(=O)c1ccccc1OC</smiles>

BIOAC

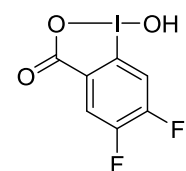

3,4-F-BIOH

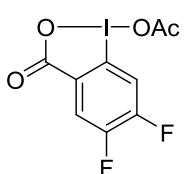

3,4-F-BIOAC<smiles>CO[Te]c1ccccc1C(=O)O</smiles>

$\mathrm{BIOMe}$ 


\section{Characterization Data:}<smiles>O=C(OCCCCC(C#CCC(F)(F)F)Cc1ccccc1)c1ccccc1</smiles>

3a: 7-phenyl-5-(2,2,2-trifluoroethyl)hept-6-yn-1-yl benzoate. Prepared according to the general procedure. Reaction was run using $1 \mathrm{a}(40.8 \mathrm{mg}, 0.2 \mathrm{mmol}), \mathrm{NaOAc}(3.3 \mathrm{mg}, 0.04 \mathrm{mmol}), \mathrm{AgOCOCF}_{3}$ (4.4 mg, $0.02 \mathrm{mmol}$ ), BIOMe (22.2 mg, $0.08 \mathrm{mmol}), \mathrm{Me}_{3} \mathrm{SiCF}_{3}(120 \mu \mathrm{L}, 0.8 \mathrm{mmol}), \mathbf{2 a}(208.8 \mathrm{mg}, 0.6$ $\mathrm{mmol}$ ) and DMSO $(1.0 \mathrm{~mL})$. The product was purified by flash column chromatography on silica gel (hexane/EtOAc) and obtained a colorless oil $(0.14 \mathrm{mmol}, 53.8 \mathrm{mg}, 72 \%), \mathrm{R}_{\mathrm{f}}=0.40$ (hexane $:$ EtOAc $=$ $8: 1) .{ }^{1} \mathbf{H}$ NMR $\left(400 \mathrm{MHz}, \mathrm{CDCl}_{3}\right): \delta 8.06(\mathrm{~d}, J=7.2 \mathrm{~Hz}, 2 \mathrm{H}), 7.56(\mathrm{t}, J=7.4 \mathrm{~Hz}, 1 \mathrm{H}), 7.43-7.38(\mathrm{~m}$, $4 \mathrm{H}), 7.29-7.25(\mathrm{~m}, 3 \mathrm{H}), 4.38(\mathrm{t}, J=6.0 \mathrm{~Hz}, 2 \mathrm{H}), 3.01-2.98(\mathrm{~m}, 1 \mathrm{H}), 2.55-2.41(\mathrm{~m}, 1 \mathrm{H}), 2.39-2.26(\mathrm{~m}$, 1H), 1.95-1.81 (m, 3H), 1.77-1.67 (m, 3H) ppm. ${ }^{13} \mathbf{C}$ NMR (101 MHz, $\left.\mathrm{CDCl}_{3}\right): \delta$ 166.7, 133.0, 131.7, $130.4,129.6,128.5,128.3,128.1,126.3$ (q, $J_{C-F}=278.5 \mathrm{~Hz}$ ), 123.3, 89.7, 83.0, 64.8, 39.3 (q, $J_{C-F}=27.9$ $\mathrm{Hz}), 34.5,28.5,26.6,23.7 \mathrm{ppm} .{ }^{19} \mathbf{F}$ NMR (376 MHz, $\left.\mathrm{CDCl}_{3}\right): \delta-65.14(\mathrm{t}, J=10.5 \mathrm{~Hz}, 3 \mathrm{~F}) . \mathbf{H R M S ~ m} / \mathrm{z}$ (APCI): calcd. for $\mathrm{C}_{22} \mathrm{H}_{21} \mathrm{~F}_{3} \mathrm{O}_{2} \mathrm{Na}[\mathrm{M}+\mathrm{Na}]^{+}$: 397.1386; found: 397.1386 .<smiles>Cc1ccc(C(=O)OCCCCC(C#CCC(F)(F)c2ccccc2)CC(F)(F)F)cc1</smiles>

3b: 7-phenyl-5-(2,2,2-trifluoroethyl)hept-6-yn-1-yl 4-methylbenzoate. Prepared according to the general procedure. Reaction was run using $1 \mathbf{b}(43.6 \mathrm{mg}, 0.2 \mathrm{mmol}), \mathrm{NaOAc}(3.3 \mathrm{mg}, 0.04 \mathrm{mmol})$, $\mathrm{AgOCOCF}_{3}(4.4 \mathrm{mg}, 0.02 \mathrm{mmol}), \mathrm{BIOMe}(22.2 \mathrm{mg}, 0.08 \mathrm{mmol}), \mathrm{Me}_{3} \mathrm{SiCF}_{3}(120 \mu \mathrm{L}, 0.8 \mathrm{mmol}), 2 \mathrm{a}$ $(208.8 \mathrm{mg}, 0.6 \mathrm{mmol})$ and DMSO $(1.0 \mathrm{~mL})$. The product was purified by flash column chromatography on silica gel (hexane/EtOAc) and obtained a colorless oil $(0.12 \mathrm{mmol}, 48.0 \mathrm{mg}, 62 \%), \mathrm{R}_{\mathrm{f}}=0.50$ (hexane : EtOAc = 8 : 1). ${ }^{1} \mathbf{H}$ NMR $\left(400 \mathrm{MHz}, \mathrm{CDCl}_{3}\right): \delta 7.96(\mathrm{~d}, J=8.0 \mathrm{~Hz}, 2 \mathrm{H}), 7.41-7.38(\mathrm{~m}, 2 \mathrm{H}), 7.32-7.27$ (m, 3H), $7.21(\mathrm{~d}, J=8.0 \mathrm{~Hz}, 2 \mathrm{H}), 4.37(\mathrm{t}, J=6.0 \mathrm{~Hz}, 2 \mathrm{H}), 3.01-2.97(\mathrm{~m}, 1 \mathrm{H}), 2.55-2.42(\mathrm{~m}, 1 \mathrm{H}), 2.42$ (s, 3H), 2.39-2.26 (m, 1H), 1.91-1.78 (m, 3H), 1.76-1.65 (m, 3H) ppm. ${ }^{13} \mathbf{C}$ NMR $\left(101 \mathrm{MHz}, \mathrm{CDCl}_{3}\right): \delta$ 166.8, 143.6, 131.7, 129.7, 129.2, 128.3, 128.1, 127.7, 126.3 (q, $J_{C-F}=278.6 \mathrm{~Hz}$ ), 123.3, 89.7, 83.0, 64.5, $39.3\left(\mathrm{q}, J_{C-F}=27.8 \mathrm{~Hz}\right), 34.3,28.5,26.6,23.7,21.7$ ppm. ${ }^{19} \mathbf{F} \mathbf{~ N M R}\left(376 \mathrm{MHz}, \mathrm{CDCl}_{3}\right): \delta-65.14(\mathrm{t}, J=$ $10.5 \mathrm{~Hz}, 3 \mathrm{~F}$ ). HRMS m/z (ESI): calcd. for $\mathrm{C}_{23} \mathrm{H}_{23} \mathrm{~F}_{3} \mathrm{O}_{2} \mathrm{Na}$ [M+Na] $]^{+}: 411.1542$; found: 411.1540 .<smiles></smiles>

3c: 7-phenyl-5-(2,2,2-trifluoroethyl)hept-6-yn-1-yl 4-methoxybenzoate. Prepared according to the general procedure. Reaction was run using 1 c $(46.8 \mathrm{mg}, 0.2 \mathrm{mmol}), \mathrm{NaOAc}(3.3 \mathrm{mg}, 0.04 \mathrm{mmol})$, $\mathrm{AgOCOCF}_{3}(4.4 \mathrm{mg}, 0.02 \mathrm{mmol}), \mathrm{BIOMe}(22.2 \mathrm{mg}, 0.08 \mathrm{mmol}), \mathrm{Me}_{3} \mathrm{SiCF}_{3}(120 \mu \mathrm{L}, 0.8 \mathrm{mmol}), 2 \mathrm{a}$ (208.8 $\mathrm{mg}, 0.6 \mathrm{mmol})$ and DMSO $(1.0 \mathrm{~mL})$. The product was purified by flash column chromatography on silica gel (hexane/EtOAc) and obtained a colorless oil $(0.15 \mathrm{mmol}, 62.2 \mathrm{mg}, 77 \%), \mathrm{R}_{\mathrm{f}}=0.20$ (hexane : EtOAc $=8: 1) .{ }^{1} \mathbf{H}$ NMR $\left(400 \mathrm{MHz}, \mathrm{CDCl}_{3}\right): \delta 8.02(\mathrm{~d}, J=9.2 \mathrm{~Hz}, 2 \mathrm{H}), 7.42-7.39(\mathrm{~m}, 2 \mathrm{H}), 7.30-7.28$ 
$(\mathrm{m}, 3 \mathrm{H}), 6.88(\mathrm{~d}, J=8.8 \mathrm{~Hz}, 2 \mathrm{H}), 4.36(\mathrm{t}, J=5.6 \mathrm{~Hz}, 2 \mathrm{H}), 3.85(\mathrm{~s}, 3 \mathrm{H}), 3.01-2.98(\mathrm{~m}, 1 \mathrm{H}), 2.56-2.42(\mathrm{~m}$, $1 \mathrm{H}), 2.39-2.26(\mathrm{~m}, 1 \mathrm{H}), 1.93-1.78(\mathrm{~m}, 3 \mathrm{H}), 1.77-1.66(\mathrm{~m}, 3 \mathrm{H}) \mathrm{ppm} .{ }^{13} \mathbf{C}$ NMR $\left(101 \mathrm{MHz}, \mathrm{CDCl}_{3}\right): \delta$ $166.5,163.4,131.7,131.6,128.3,128.1,126.3$ (q, $\left.J_{C-F}=278.5 \mathrm{~Hz}\right), 123.3,122.9,113.7,89.7,83.0,64.4$, $55.5,39.2\left(\mathrm{q}, J_{C-F}=27.8 \mathrm{~Hz}\right), 34.4,28.5,26.5,23.7 \mathrm{ppm} .{ }^{19} \mathbf{F} \mathbf{~ N M R}\left(376 \mathrm{MHz}, \mathrm{CDCl}_{3}\right): \delta-65.12(\mathrm{t}, J=$ $10.5 \mathrm{~Hz}, 3 \mathrm{~F})$. HRMS m/z (APCI): calcd. for $\mathrm{C}_{23} \mathrm{H}_{23} \mathrm{~F}_{3} \mathrm{O}_{3} \mathrm{Na}[\mathrm{M}+\mathrm{Na}]^{+}: 427.1492$; found: 427.1492 .<smiles>COc1cccc(C(=O)OCCCCC(C#Cc2ccccc2)CC(F)(F)F)c1</smiles>

3d: 7-phenyl-5-(2,2,2-trifluoroethyl)hept-6-yn-1-yl 3-methoxybenzoate. Prepared according to the general procedure. Reaction was run using 1 d $(46.8 \mathrm{mg}, 0.2 \mathrm{mmol}), \mathrm{NaOAc}(3.3 \mathrm{mg}, 0.04 \mathrm{mmol})$, $\mathrm{AgOCOCF}_{3}(4.4 \mathrm{mg}, 0.02 \mathrm{mmol}), \mathrm{BIOMe}(22.2 \mathrm{mg}, 0.08 \mathrm{mmol}), \mathrm{Me}_{3} \mathrm{SiCF}_{3}(120 \mu \mathrm{L}, 0.8 \mathrm{mmol}), \mathbf{2 a}$ $(208.8 \mathrm{mg}, 0.6 \mathrm{mmol})$ and DMSO $(1.0 \mathrm{~mL})$. The product was purified by flash column chromatography on silica gel (hexane/EtOAc) and obtained a colorless oil ( $0.13 \mathrm{mmol}, 50.9 \mathrm{mg}, 63 \%), \mathrm{R}_{\mathrm{f}}=0.20$ (hexane : EtOAc $=8: 1) .{ }^{1} \mathbf{H}$ NMR $\left(400 \mathrm{MHz}, \mathrm{CDCl}_{3}\right): \delta 7.66(\mathrm{~d}, J=7.6 \mathrm{~Hz}, 1 \mathrm{H}), 7.59(\mathrm{~s}, 2 \mathrm{H}), 7.40-7.38(\mathrm{~m}, 2 \mathrm{H})$, 7.34-7.28 (m, 4H), $7.11(\mathrm{dd}, J=8.4 \mathrm{~Hz}, J=2.0 \mathrm{~Hz}, 2 \mathrm{H}), 4.38$ (t, $J=6.2 \mathrm{~Hz}, 2 \mathrm{H}), 3.84$ (s, 3H), 3.01-2.98 $(\mathrm{m}, 1 \mathrm{H}), 2.55-2.41(\mathrm{~m}, 1 \mathrm{H}), 2.39-2.27(\mathrm{~m}, 1 \mathrm{H}), 1.93-1.77(\mathrm{~m}, 3 \mathrm{H}), 1.77-1.66(\mathrm{~m}, 3 \mathrm{H}) \mathrm{ppm} .{ }^{13} \mathbf{C}$ NMR $\left(101 \mathrm{MHz}, \mathrm{CDCl}_{3}\right): \delta 166.6,159.7,131.8,131.7,129.5,128.3,128.1,126.3$ (q, $\left.J_{C-F}=278.5 \mathrm{~Hz}\right), 123.3$, 122.1, 119.4, 114.1, 89.7, 83.0, 64.9, 55.5, 39.3 (q, $\left.J_{C-F}=27.9 \mathrm{~Hz}\right), 34.4,28.5,26.6,23.7$ ppm. ${ }^{19} \mathbf{F}$ NMR $\left(376 \mathrm{MHz}, \mathrm{CDCl}_{3}\right): \delta-65.14$ (t, $\left.J=10.7 \mathrm{~Hz}, 3 \mathrm{~F}\right) . \mathbf{H R M S ~ m} / \mathrm{z}(\mathrm{ESI}):$ calcd. for $\mathrm{C}_{23} \mathrm{H}_{23} \mathrm{~F}_{3} \mathrm{O}_{3} \mathrm{Na}[\mathrm{M}+\mathrm{Na}]^{+}$: 427.1492; found: 427.1492 .<smiles>CCCC#CC(CCCCOC(=O)c1ccccc1OC)Cc1ccccc1</smiles>

3e: 7-phenyl-5-(2,2,2-trifluoroethyl)hept-6-yn-1-yl 2-methoxybenzoate. Prepared according to the general procedure. Reaction was run using 1e $(46.8 \mathrm{mg}, 0.2 \mathrm{mmol}), \mathrm{NaOAc}(3.3 \mathrm{mg}, 0.04 \mathrm{mmol})$, $\mathrm{AgOCOCF}_{3}(4.4 \mathrm{mg}, 0.02 \mathrm{mmol}), \mathrm{BIOMe}(22.2 \mathrm{mg}, 0.08 \mathrm{mmol}), \mathrm{Me}_{3} \mathrm{SiCF}_{3}(120 \mu \mathrm{L}, 0.8 \mathrm{mmol}), 2 \mathrm{a}$ (208.8 $\mathrm{mg}, 0.6 \mathrm{mmol})$ and DMSO $(1.0 \mathrm{~mL})$. The product was purified by flash column chromatography on silica gel (hexane/EtOAc) and obtained a colorless oil ( $0.15 \mathrm{mmol}, 59.0 \mathrm{mg}, 73 \%), \mathrm{R}_{\mathrm{f}}=0.20$ (hexane : EtOAc $=8: 1) .{ }^{1} \mathbf{H}$ NMR $\left(400 \mathrm{MHz}, \mathrm{CDCl}_{3}\right): \delta 7.81(\mathrm{dd}, J=7.6 \mathrm{~Hz}, J=2.0 \mathrm{~Hz}, 1 \mathrm{H}), 7.47(\mathrm{dt}, J=7.8$ $\mathrm{Hz}, J=1.6 \mathrm{~Hz}, 1 \mathrm{H}), 7.41-7.38(\mathrm{~m}, 2 \mathrm{H}), 7.32-7.26(\mathrm{~m}, 3 \mathrm{H}), 6.98-6.92(\mathrm{~m}, 2 \mathrm{H}), 4.36(\mathrm{t}, J=6.0 \mathrm{~Hz}, 2 \mathrm{H})$, $3.88(\mathrm{~s}, 3 \mathrm{H}), 2.98-2.96(\mathrm{~m}, 1 \mathrm{H}), 2.55-2.41(\mathrm{~m}, 1 \mathrm{H}), 2.39-2.26(\mathrm{~m}, 1 \mathrm{H}), 1.90-1.78(\mathrm{~m}, 3 \mathrm{H}), 1.75-1.66(\mathrm{~m}$, $3 \mathrm{H}) \mathrm{ppm} .{ }^{13} \mathbf{C}$ NMR $\left(101 \mathrm{MHz}, \mathrm{CDCl}_{3}\right): \delta 166.4,159.2,133.5,131.7,131.6,128.3,128.1,126.3\left(\mathrm{q}, J_{C-}\right.$ $\left.{ }_{F}=278.6 \mathrm{~Hz}\right), 123.3,120.3,120.2,112.1,89.8,82.9,64.6,56.0,39.2\left(\mathrm{q}, J_{C-F}=27.8 \mathrm{~Hz}\right), 34.5,28.4,26.6$, 23.7 ppm. ${ }^{19} \mathbf{F}$ NMR $\left(376 \mathrm{MHz}, \mathrm{CDCl}_{3}\right): \delta-65.15$ (t, $\left.J=10.5 \mathrm{~Hz}, 3 \mathrm{~F}\right)$. HRMS m/z (ESI): calcd. for $\mathrm{C}_{23} \mathrm{H}_{23} \mathrm{~F}_{3} \mathrm{O}_{3} \mathrm{Na}[\mathrm{M}+\mathrm{Na}]^{+}:$427.1492; found: 427.1492 .<smiles>O=C(OCCCCC(C#CC(F)(F)F)Cc1ccccc1)c1ccc(C(F)(F)F)cc1</smiles> 
3f: 7-phenyl-5-(2,2,2-trifluoroethyl)hept-6-yn-1-yl 4-(trifluoromethyl)benzoate. Prepared according to the general procedure. Reaction was run using $\mathbf{1 f}(54.4 \mathrm{mg}, 0.2 \mathrm{mmol})$, NaOAc (3.3 $\mathrm{mg}, 0.04 \mathrm{mmol})$, $\mathrm{AgOCOCF}_{3}$ (4.4 mg, $0.02 \mathrm{mmol}$ ), BIOMe (22.2 mg, $\left.0.08 \mathrm{mmol}\right), \mathrm{Me}_{3} \mathrm{SiCF}_{3}(120 \mu \mathrm{L}, 0.8 \mathrm{mmol}), 2 \mathrm{a}$ (208.8 mg, $0.6 \mathrm{mmol})$ and DMSO $(1.0 \mathrm{~mL})$. The product was purified by flash column chromatography on silica gel (hexane/EtOAc) and obtained a colorless oil ( $0.14 \mathrm{mmol}, 60.1 \mathrm{mg}, 68 \%$ ), $\mathrm{R}_{\mathrm{f}}=0.50$ (hexane : EtOAc $=8: 1) .{ }^{1} \mathbf{H}$ NMR $\left(400 \mathrm{MHz}, \mathrm{CDCl}_{3}\right): \delta 8.15(\mathrm{~d}, J=8.4 \mathrm{~Hz}, 2 \mathrm{H}), 7.63(\mathrm{~d}, J=8.4 \mathrm{~Hz}, 2 \mathrm{H}), 7.40$ $7.37(\mathrm{~m}, 2 \mathrm{H}), 7.31-7.26(\mathrm{~m}, 3 \mathrm{H}), 4.42(\mathrm{t}, J=5.6 \mathrm{~Hz}, 2 \mathrm{H}), 3.01-2.98(\mathrm{~m}, 1 \mathrm{H}), 2.57-2.43(\mathrm{~m}, 1 \mathrm{H}), 2.38-$ $2.28(\mathrm{~m}, 1 \mathrm{H}), 1.95-1.81(\mathrm{~m}, 3 \mathrm{H}), 1.78-1.65(\mathrm{~m}, 3 \mathrm{H}) \mathrm{ppm} .{ }^{13} \mathbf{C}$ NMR $\left(101 \mathrm{MHz}, \mathrm{CDCl}_{3}\right): \delta 166.5,134.4$ $\left(\mathrm{q}, J_{C-F}=32.7 \mathrm{~Hz}\right), 133.6,131.7,130.0,128.4,128.2,126.3\left(\mathrm{q}, J_{C-F}=278.5 \mathrm{~Hz}\right), 125.5\left(\mathrm{q}, J_{C-F}=3.7 \mathrm{~Hz}\right)$, $123.7\left(\mathrm{q}, J_{C-F}=273.7 \mathrm{~Hz}\right), 123.2,89.6,83.1,65.2,39.3\left(\mathrm{q}, J_{C-F}=27.8 \mathrm{~Hz}\right), 34.4,28.3,26.6,23.6 \mathrm{ppm}$. ${ }^{19}$ F NMR $\left(376 \mathrm{MHz}, \mathrm{CDCl}_{3}\right): \delta-64.14(\mathrm{~s}, 3 \mathrm{~F}),-65.13$ (t, $\left.J=10.7 \mathrm{~Hz}, 3 \mathrm{~F}\right)$. HRMS m/z (APCI): calcd. for $\mathrm{C}_{23} \mathrm{H}_{21} \mathrm{~F}_{6} \mathrm{O}_{2}[\mathrm{M}+\mathrm{H}]^{+}:$443.1440; found: 443.1439 .<smiles>N#Cc1ccc(C(=O)OCCCCC(C#Cc2ccccc2)CC(F)(F)F)cc1</smiles>

3g: 7-phenyl-5-(2,2,2-trifluoroethyl)hept-6-yn-1-yl 4-cyanobenzoate. Prepared according to the general procedure. Reaction was run using $1 \mathrm{~g}(45.8 \mathrm{mg}, 0.2 \mathrm{mmol}), \mathrm{NaOAc}(3.3 \mathrm{mg}, 0.04 \mathrm{mmol})$, $\mathrm{AgOCOCF}_{3}$ (4.4 mg, $0.02 \mathrm{mmol}$ ), BIOMe (22.2 mg, $\left.0.08 \mathrm{mmol}\right), \mathrm{Me}_{3} \mathrm{SiCF}_{3}(120 \mu \mathrm{L}, 0.8 \mathrm{mmol}), 2 \mathrm{a}$ $(208.8 \mathrm{mg}, 0.6 \mathrm{mmol})$ and DMSO $(1.0 \mathrm{~mL})$. The product was purified by flash column chromatography on silica gel (hexane/EtOAc) and obtained a colorless oil ( $0.14 \mathrm{mmol}, 55.9 \mathrm{mg}, 70 \%), \mathrm{R}_{\mathrm{f}}=0.50$ (hexane : EtOAc $=5: 1) .{ }^{1} \mathbf{H}$ NMR $\left(400 \mathrm{MHz}, \mathrm{CDCl}_{3}\right): \delta 8.11(\mathrm{~d}, J=8.8 \mathrm{~Hz}, 2 \mathrm{H}), 7.64(\mathrm{~d}, J=8.0 \mathrm{~Hz}, 2 \mathrm{H}), 7.38-$ $7.33(\mathrm{~m}, 2 \mathrm{H}), 7.32-7.26(\mathrm{~m}, 3 \mathrm{H}), 4.41$ (t, $J=6.0 \mathrm{~Hz}, 2 \mathrm{H}), 3.02-2.95(\mathrm{~m}, 1 \mathrm{H}), 2.55-2.41(\mathrm{~m}, 1 \mathrm{H}), 2.37-$ $2.27(\mathrm{~m}, 1 \mathrm{H}), 1.94-1.77(\mathrm{~m}, 3 \mathrm{H}), 1.77-1.65(\mathrm{~m}, 3 \mathrm{H}) \mathrm{ppm} .{ }^{13} \mathbf{C} \mathbf{N M R}\left(101 \mathrm{MHz}, \mathrm{CDCl}_{3}\right): \delta 165.1,134.2$, $132.3,131.6,130.1,128.4,128.2,126.2\left(\mathrm{q}, J_{C-F}=278.5 \mathrm{~Hz}\right), 123.2,118.1,116.4,89.6,83.0,65.5,39.3$ $\left(\mathrm{q}, J_{C-F}=27.8 \mathrm{~Hz}\right), 34.3,28.3,26.6,23.6 \mathrm{ppm} .{ }^{19} \mathbf{F} \mathbf{N M R}\left(376 \mathrm{MHz}, \mathrm{CDCl}_{3}\right): \delta-65.15(\mathrm{t}, J=10.7 \mathrm{~Hz}$, $3 F)$. HRMS m/z (ESI): calcd. for $\mathrm{C}_{23} \mathrm{H}_{21} \mathrm{~F}_{3} \mathrm{NO}_{2}[\mathrm{M}+\mathrm{H}]^{+}: 400.1519$; found: 400.1516 .<smiles>O=C(OCCCCC(C#Cc1ccccc1)CC(F)(F)F)c1ccc(F)cc1</smiles>

3h: 7-phenyl-5-(2,2,2-trifluoroethyl)hept-6-yn-1-yl 4-fluorobenzoate. Prepared according to the general procedure. Reaction was run using $\mathbf{1 h}(44.4 \mathrm{mg}, 0.2 \mathrm{mmol}), \mathrm{NaOAc}(3.3 \mathrm{mg}, 0.04 \mathrm{mmol})$, $\mathrm{AgOCOCF}_{3}$ (4.4 mg, $0.02 \mathrm{mmol}$ ), BIOMe (22.2 mg, $\left.0.08 \mathrm{mmol}\right), \mathrm{Me}_{3} \mathrm{SiCF}_{3}(120 \mu \mathrm{L}, 0.8 \mathrm{mmol}), 2 \mathrm{a}$ (208.8 mg, $0.6 \mathrm{mmol})$ and DMSO $(1.0 \mathrm{~mL})$. The product was purified by flash column chromatography on silica gel (hexane/EtOAc) and obtained a colorless oil ( $0.14 \mathrm{mmol}, 56.4 \mathrm{mg}, 72 \%), \mathrm{R}_{\mathrm{f}}=0.50$ (hexane : EtOAc $=8: 1) .{ }^{1} \mathbf{H}$ NMR $\left(400 \mathrm{MHz}, \mathrm{CDCl}_{3}\right): \delta 8.07(\mathrm{~d}, J=8.8 \mathrm{~Hz}, 1 \mathrm{H}), 8.05(\mathrm{~d}, J=8.8 \mathrm{~Hz}, 1 \mathrm{H}), 7.39$ $(\mathrm{dd}, J=9.6 \mathrm{~Hz}, J=2.0 \mathrm{~Hz}, 2 \mathrm{H}), 7.32-7.27(\mathrm{~m}, 3 \mathrm{H}), 7.06(\mathrm{t}, J=8.8 \mathrm{~Hz}, 2 \mathrm{H}), 4.38(\mathrm{t}, J=5.6 \mathrm{~Hz}, 2 \mathrm{H})$, 3.01-2.98 (m, 1H), 2.56-2.42 (m, 1H), 2.38-2.28 (m, 1H), 1.92-1.78 (m, 3H), 1.75-1.64 (m, 3H) ppm. ${ }^{13}$ C NMR $\left(101 \mathrm{MHz}, \mathrm{CDCl}_{3}\right): \delta 165.8\left(\mathrm{~d}, J_{C-F}=254.6 \mathrm{~Hz}\right), 166.7,132.2\left(\mathrm{~d}, J_{C-F}=9.3 \mathrm{~Hz}\right), 131.7,128.4$, $128.1,126.7\left(\mathrm{~d}, J_{C-F}=3.0 \mathrm{~Hz}\right), 126.3\left(\mathrm{q}, J_{C-F}=278.5 \mathrm{~Hz}\right), 123.3,115.6\left(\mathrm{q}, J_{C-F}=22.0 \mathrm{~Hz}\right), 89.7,83.0$, 64.9, $39.3\left(\mathrm{q}, J_{C-F}=27.8 \mathrm{~Hz}\right), 34.4,28.4,26.6,23.7$ ppm. ${ }^{19} \mathbf{F} \mathbf{N M R}\left(376 \mathrm{MHz}, \mathrm{CDCl}_{3}\right): \delta-65.12(\mathrm{t}, J=$ 
$10.7 \mathrm{~Hz}, 3 \mathrm{~F}),-106.9(\mathrm{~m}, 1 \mathrm{~F})$. HRMS m/z (ESI): calcd. for $\mathrm{C}_{22} \mathrm{H}_{20} \mathrm{~F}_{4} \mathrm{O}_{2} \mathrm{Na}[\mathrm{M}+\mathrm{Na}]^{+}$: 415.1292 ; found: 415.1290 .<smiles>O=C(OCCCCC(C#Cc1ccccc1)CC(F)(F)F)c1ccc(Cl)cc1</smiles>

3i: 7-phenyl-5-(2,2,2-trifluoroethyl)hept-6-yn-1-yl 4-chlorobenzoate. Prepared according to the general procedure. Reaction was run using $1 \mathbf{i}(47.6 \mathrm{mg}, 0.2 \mathrm{mmol}), \mathrm{NaOAc}(3.3 \mathrm{mg}, 0.04 \mathrm{mmol})$, $\mathrm{AgOCOCF}_{3}$ (4.4 mg, $\left.0.02 \mathrm{mmol}\right), \mathrm{BIOMe}(22.2 \mathrm{mg}, 0.08 \mathrm{mmol}), \mathrm{Me}_{3} \mathrm{SiCF}_{3}(120 \mu \mathrm{L}, 0.8 \mathrm{mmol}), 2 \mathrm{a}$ $(208.8 \mathrm{mg}, 0.6 \mathrm{mmol})$ and DMSO $(1.0 \mathrm{~mL})$. The product was purified by flash column chromatography on silica gel (hexane/EtOAc) and obtained a colorless oil ( $0.14 \mathrm{mmol}, 57.1 \mathrm{mg}, 70 \%), \mathrm{R}_{\mathrm{f}}=0.50$ (hexane : EtOAc = $8:$ 1). ${ }^{1} \mathbf{H}$ NMR $\left(400 \mathrm{MHz}, \mathrm{CDCl}_{3}\right): \delta 7.97(\mathrm{~d}, J=8.4 \mathrm{~Hz}, 2 \mathrm{H}), 7.38-7.34(\mathrm{~m}, 4 \mathrm{H}), 7.31-7.27$ $(\mathrm{m}, 3 \mathrm{H}), 4.37$ (t, $J=5.6 \mathrm{~Hz}, 2 \mathrm{H}), 3.01-2.97(\mathrm{~m}, 1 \mathrm{H}), 2.54-2.41(\mathrm{~m}, 1 \mathrm{H}), 2.38-2.25(\mathrm{~m}, 1 \mathrm{H}), 1.91-1.78$ $(\mathrm{m}, 3 \mathrm{H}), 1.76-1.64(\mathrm{~m}, 3 \mathrm{H}) \mathrm{ppm} .{ }^{13} \mathbf{C}$ NMR (101 MHz, $\left.\mathrm{CDCl}_{3}\right): \delta 165.9,139.4,131.7,131.0,128.9$, $128.8,128.4,128.2,126.3\left(\mathrm{q}, J_{C-F}=278.6 \mathrm{~Hz}\right), 123.2,89.7,83.0,65.0,39.3\left(\mathrm{q}, J_{C-F}=27.9 \mathrm{~Hz}\right), 34.4$, 28.4, 26.6, 23.7 ppm. ${ }^{19} \mathbf{F}$ NMR (376 MHz, $\left.\mathrm{CDCl}_{3}\right): \delta-65.14(\mathrm{t}, J=10.7 \mathrm{~Hz}, 3 \mathrm{~F}) . \mathbf{H R M S ~ m} / \mathrm{z}$ (ESI): calcd. for $\mathrm{C}_{22} \mathrm{H}_{20} \mathrm{ClF}_{3} \mathrm{O}_{2} \mathrm{Na}[\mathrm{M}+\mathrm{Na}]^{+}$: 431.0996; found: 431.0997 .<smiles>O=C(OCCCCC(C#CPc1ccccc1)CC(F)(F)F)c1ccc(Br)cc1</smiles>

3j: 7-phenyl-5-(2,2,2-trifluoroethyl)hept-6-yn-1-yl 4-bromobenzoate. Prepared according to the general procedure. Reaction was run using 1j (56.4 mg, $0.2 \mathrm{mmol}), \mathrm{NaOAc}(3.3 \mathrm{mg}, 0.04 \mathrm{mmol})$, $\mathrm{AgOCOCF}_{3}$ (4.4 mg, $0.02 \mathrm{mmol}$ ), BIOMe (22.2 mg, $\left.0.08 \mathrm{mmol}\right), \mathrm{Me}_{3} \mathrm{SiCF}_{3}(120 \mu \mathrm{L}, 0.8 \mathrm{mmol}), 2 \mathrm{a}$ (208.8 $\mathrm{mg}, 0.6 \mathrm{mmol})$ and DMSO $(1.0 \mathrm{~mL})$. The product was purified by flash column chromatography on silica gel (hexane/EtOAc) and obtained a colorless oil ( $0.16 \mathrm{mmol}, 72.3 \mathrm{mg}, 80 \%), \mathrm{R}_{\mathrm{f}}=0.50$ (hexane : EtOAc $=8: 1) .{ }^{1} \mathbf{H}$ NMR $\left(400 \mathrm{MHz}, \mathrm{CDCl}_{3}\right): \delta 7.90(\mathrm{~d}, J=8.4 \mathrm{~Hz}, 2 \mathrm{H}), 7.52(\mathrm{~d}, J=8.8 \mathrm{~Hz}, 2 \mathrm{H}), 7.40$ $7.36(\mathrm{~m}, 2 \mathrm{H}), 7.32-7.27(\mathrm{~m}, 3 \mathrm{H}), 4.38(\mathrm{t}, J=5.6 \mathrm{~Hz}, 2 \mathrm{H}), 3.01-2.97(\mathrm{~m}, 1 \mathrm{H}), 2.55-2.42(\mathrm{~m}, 1 \mathrm{H}), 2.39-$ $2.26(\mathrm{~m}, 1 \mathrm{H}), 1.91-1.78(\mathrm{~m}, 3 \mathrm{H}), 1.76-1.64(\mathrm{~m}, 3 \mathrm{H}) \mathrm{ppm} .{ }^{13} \mathbf{C} \mathbf{N M R}\left(101 \mathrm{MHz}, \mathrm{CDCl}_{3}\right): \delta 166.0,131.8$, $131.7,131.2,129.3,128.4,128.2,128.1,126.3\left(\mathrm{q}, J_{C-F}=278.6 \mathrm{~Hz}\right), 123.2,89.6,83.0,65.0,39.3\left(\mathrm{q}, J_{C-}\right.$ $\left.{ }_{F}=27.8 \mathrm{~Hz}\right), 34.3,28.4,26.6,23.6$ ppm. ${ }^{19} \mathbf{F}$ NMR $\left(376 \mathrm{MHz}, \mathrm{CDCl}_{3}\right): \delta-65.13(\mathrm{t}, J=10.5 \mathrm{~Hz}, 3 \mathrm{~F})$. HRMS m/z (ESI): calcd. for $\mathrm{C}_{22} \mathrm{H}_{20} \mathrm{BrF}_{3} \mathrm{O}_{2} \mathrm{Na}[\mathrm{M}+\mathrm{Na}]^{+}: 475.0491$; found: 475.0494 .<smiles>O=C(OCCCCC(C#CPc1ccccc1)CC(F)(F)F)c1ccc(I)cc1</smiles>

3k: 7-phenyl-5-(2,2,2-trifluoroethyl)hept-6-yn-1-yl 4-Iodobenzoate. Prepared according to the general procedure. Reaction was run using $1 \mathbf{k}(66.0 \mathrm{mg}, 0.2 \mathrm{mmol}), \mathrm{NaOAc}(3.3 \mathrm{mg}, 0.04 \mathrm{mmol})$, $\mathrm{AgOCOCF}_{3}$ (4.4 mg, $\left.0.02 \mathrm{mmol}\right), \mathrm{BIOMe}(22.2 \mathrm{mg}, 0.08 \mathrm{mmol}), \mathrm{Me}_{3} \mathrm{SiCF}_{3}(120 \mu \mathrm{L}, 0.8 \mathrm{mmol}), 2 \mathrm{a}$ (208.8 $\mathrm{mg}, 0.6 \mathrm{mmol})$ and DMSO $(1.0 \mathrm{~mL})$. The product was purified by flash column chromatography on silica gel (hexane/EtOAc) and obtained a colorless oil ( $0.13 \mathrm{mmol}, 67.0 \mathrm{mg}, 67 \%), \mathrm{R}_{\mathrm{f}}=0.50$ (hexane : 
EtOAc $=8: 1) .{ }^{1} \mathbf{H} \mathbf{N M R}\left(400 \mathrm{MHz}, \mathrm{CDCl}_{3}\right): \delta$ 7.78-7.70 (m, 4H), 7.38-7.36 (m, 2H), 7.32-7.27 (m, 3H), $4.37(\mathrm{t}, J=5.6 \mathrm{~Hz}, 2 \mathrm{H}), 3.01-2.97(\mathrm{~m}, 1 \mathrm{H}), 2.55-2.41(\mathrm{~m}, 1 \mathrm{H}), 2.38-2.26(\mathrm{~m}, 1 \mathrm{H}), 1.91-1.77(\mathrm{~m}, 3 \mathrm{H})$, 1.75-1.63 (m, 3H) ppm. ${ }^{13} \mathbf{C}$ NMR (101 MHz, $\left.\mathrm{CDCl}_{3}\right): \delta 166.2,137.8,131.7,131.1,129.9,128.4,128.2$, 126.3 (q, $\left.J_{C-F}=278.6 \mathrm{~Hz}\right), 123.2,100.8,89.6,83.0,64.9,39.3$ (q, $\left.J_{C-F}=27.8 \mathrm{~Hz}\right), 34.3,28.4,26.5,23.6$ ppm. ${ }^{19} \mathbf{F}$ NMR $\left(376 \mathrm{MHz}, \mathrm{CDCl}_{3}\right): \delta-65.14$ (t, $\left.J=10.5 \mathrm{~Hz}, 3 \mathrm{~F}\right)$. HRMS m/z (ESI): calcd. for $\mathrm{C}_{22} \mathrm{H}_{20} \mathrm{~F}_{3} \mathrm{IO}_{2} \mathrm{Na}[\mathrm{M}+\mathrm{Na}]^{+}$: 523.0352; found: 523.0354.<smiles>O=C(OCCCCC(C#CCC(F)(F)F)Cc1ccco1)c1ccccc1</smiles>

31: 7-phenyl-5-(2,2,2-trifluoroethyl)hept-6-yn-1-yl furan-2-carboxylate. Prepared according to the general procedure. Reaction was run using 11 (38.8 mg, $0.2 \mathrm{mmol})$, NaOAc $(3.3 \mathrm{mg}, 0.04 \mathrm{mmol})$, $\mathrm{AgOCOCF}_{3}(4.4 \mathrm{mg}, 0.02 \mathrm{mmol}), \mathrm{BIOMe}(22.2 \mathrm{mg}, 0.08 \mathrm{mmol}), \mathrm{Me}_{3} \mathrm{SiCF}_{3}(120 \mu \mathrm{L}, 0.8 \mathrm{mmol}), \mathbf{2 a}$ $(208.8 \mathrm{mg}, 0.6 \mathrm{mmol})$ and DMSO $(1.0 \mathrm{~mL})$. The product was purified by flash column chromatography on silica gel (hexane/EtOAc) and obtained a colorless oil ( $0.10 \mathrm{mmol}, 37.9 \mathrm{mg}, 52 \%), \mathrm{R}_{\mathrm{f}}=0.60$ (hexane : EtOAc $=8: 1) .{ }^{1} \mathbf{H}$ NMR $\left(400 \mathrm{MHz}, \mathrm{CDCl}_{3}\right): \delta 7.56(\mathrm{~s}, 1 \mathrm{H}), 7.40-7.38(\mathrm{~m}, 2 \mathrm{H}), 7.30-7.27(\mathrm{~m}, 3 \mathrm{H}), 7.16$ $(\mathrm{d}, J=3.2 \mathrm{~Hz}, 1 \mathrm{H}), 6.48(\mathrm{dd}, J=1.6 \mathrm{~Hz}, 1 \mathrm{H}), 4.36(\mathrm{t}, J=6.2 \mathrm{~Hz}, 2 \mathrm{H}), 3.00-2.94(\mathrm{~m}, 1 \mathrm{H}), 2.53-2.39(\mathrm{~m}$, $1 \mathrm{H}), 2.37-2.25(\mathrm{~m}, 1 \mathrm{H}), 1.88-1.74(\mathrm{~m}, 3 \mathrm{H}), 1.74-1.62(\mathrm{~m}, 3 \mathrm{H}) \mathrm{ppm} .{ }^{13} \mathbf{C}$ NMR $\left(101 \mathrm{MHz}, \mathrm{CDCl}_{3}\right): \delta$ 158.9, 146.4, 144.8, 131.7, 128.3, 128.1, 126.3 (q, $J_{C-F}=278.4 \mathrm{~Hz}$ ), 123.3, 117.9, 111.9, 89.7, 83.0, 64.7, $39.3\left(\mathrm{q}, J_{C-F}=27.9 \mathrm{~Hz}\right), 34.4,28.4,26.5,23.6 \mathrm{ppm} .{ }^{19} \mathbf{F} \mathbf{~ N M R}\left(376 \mathrm{MHz}, \mathrm{CDCl}_{3}\right): \delta-65.15(\mathrm{t}, J=10.7$ $\mathrm{Hz}, 3 \mathrm{~F})$. HRMS m/z (ESI): calcd. for $\mathrm{C}_{20} \mathrm{H}_{19} \mathrm{~F}_{3} \mathrm{O}_{3} \mathrm{Na}[\mathrm{M}+\mathrm{Na}]^{+}: 387.1179$; found: 387.1176 .

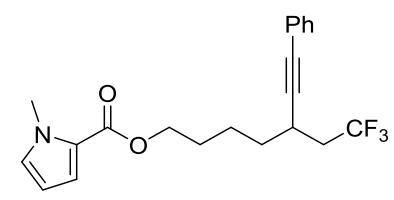

3m: 7-phenyl-5-(2,2,2-trifluoroethyl)hept-6-yn-1-yl 1-methyl-1H-pyrrole-2-carboxylate. Prepared according to the general procedure. Reaction was run using $1 \mathbf{m}$ ( $41.4 \mathrm{mg}, 0.2 \mathrm{mmol}), \mathrm{NaOAc}$ (3.3 $\mathrm{mg}$, $0.04 \mathrm{mmol}$ ), $\mathrm{AgOCOCF}_{3}(4.4 \mathrm{mg}, 0.02 \mathrm{mmol}), \mathrm{BIOMe}(22.2 \mathrm{mg}, 0.08 \mathrm{mmol}), \mathrm{Me}_{3} \mathrm{SiCF}_{3}(120 \mu \mathrm{L}, 0.8$ $\mathrm{mmol}), 2 \mathrm{a}(208.8 \mathrm{mg}, 0.6 \mathrm{mmol})$ and DMSO $(1.0 \mathrm{~mL})$. The product was purified by flash column chromatography on silica gel (hexane/EtOAc) and obtained a colorless oil ( $0.10 \mathrm{mmol}, 37.7 \mathrm{mg}, 50 \%)$, $\mathrm{R}_{\mathrm{f}}=0.40$ (hexane : EtOAc $=8: 1$ ). ${ }^{1} \mathbf{H}$ NMR $\left(400 \mathrm{MHz}, \mathrm{CDCl}_{3}\right): \delta 7.40-7.38(\mathrm{~m}, 2 \mathrm{H}), 7.30-7.26(\mathrm{~m}$, $3 \mathrm{H}), 6.95(\mathrm{dd}, J=2.0 \mathrm{~Hz}, 1 \mathrm{H}), 6.77(\mathrm{t}, J=2.0 \mathrm{~Hz}, 1 \mathrm{H}), 6.09(\mathrm{~m}, 1 \mathrm{H}), 4.27(\mathrm{t}, J=6.0 \mathrm{~Hz}, 2 \mathrm{H}), 3.91(\mathrm{~s}$, $3 \mathrm{H}), 3.00-2.94(\mathrm{~m}, 1 \mathrm{H}), 2.53-2.39(\mathrm{~m}, 1 \mathrm{H}), 2.37-2.24(\mathrm{~m}, 1 \mathrm{H}), 1.86-1.74(\mathrm{~m}, 3 \mathrm{H}), 1.74-1.63(\mathrm{~m}, 3 \mathrm{H})$ ppm. ${ }^{13} \mathrm{C}$ NMR (101 MHz, $\left.\mathrm{CDCl}_{3}\right): \delta 161.5,131.7,130.4,129.6,128.4,128.1,126.3$ (q, $J_{C-F}=278.6$ $\mathrm{Hz}), 123.3,117.9,107.9,89.8,83.0,63.5,39.3$ (q, $\left.J_{C-F}=27.8 \mathrm{~Hz}\right), 36.9,34.5,28.6,26.6,23.7$ ppm. ${ }^{19} \mathbf{F}$ NMR ( $\left.376 \mathrm{MHz}, \mathrm{CDCl}_{3}\right): \delta-65.15(\mathrm{t}, J=10.5 \mathrm{~Hz}, 3 \mathrm{~F})$. HRMS m/z (ESI): calcd. for $\mathrm{C}_{21} \mathrm{H}_{22} \mathrm{~F}_{3} \mathrm{NO}_{2} \mathrm{Na}$ $[\mathrm{M}+\mathrm{Na}]^{+}:$400.1495; found: 400.1492 .<smiles>C#CC(CCCCOC(=O)c1cccs1)CC(F)(F)F</smiles> 
3n: 7-phenyl-5-(2,2,2-trifluoroethyl)hept-6-yn-1-yl thiophene-2-carboxylate. Prepared according to the general procedure. Reaction was run using $1 \mathrm{n}$ (42.0 $\mathrm{mg}, 0.2 \mathrm{mmol}), \mathrm{NaOAc}(3.3 \mathrm{mg}, 0.04 \mathrm{mmol})$, $\mathrm{AgOCOCF}_{3}$ (4.4 mg, $0.02 \mathrm{mmol}$ ), BIOMe (22.2 mg, $\left.0.08 \mathrm{mmol}\right), \mathrm{Me}_{3} \mathrm{SiCF}_{3}(120 \mu \mathrm{L}, 0.8 \mathrm{mmol}), 2 \mathrm{a}$ (208.8 mg, $0.6 \mathrm{mmol})$ and DMSO $(1.0 \mathrm{~mL})$. The product was purified by flash column chromatography on silica gel (hexane/EtOAc) and obtained a colorless oil $(0.14 \mathrm{mmol}, 54.7 \mathrm{mg}, 72 \%), \mathrm{R}_{\mathrm{f}}=0.50$ (hexane : EtOAc $=8: 1) .{ }^{1} \mathbf{H}$ NMR $\left(500 \mathrm{MHz}, \mathrm{CDCl}_{3}\right): \delta 7.81(\mathrm{dd}, J=3.5 \mathrm{~Hz}, J=1.0 \mathrm{~Hz}, 1 \mathrm{H}), 7.23(\mathrm{dd}, J=5.0$ $\mathrm{Hz}, J=1.0 \mathrm{~Hz}, 1 \mathrm{H}), 7.41-7.39(\mathrm{~m}, 2 \mathrm{H}), 7.31-7.27$ (m, 3H), $7.08(\mathrm{~m}, 1 \mathrm{H}), 4.36(\mathrm{t}, J=6.3 \mathrm{~Hz}, 2 \mathrm{H}), 3.02-$ $2.96(\mathrm{~m}, 1 \mathrm{H}), 2.54-2.42(\mathrm{~m}, 1 \mathrm{H}), 2.38-2.27(\mathrm{~m}, 1 \mathrm{H}), 1.89-1.78(\mathrm{~m}, 3 \mathrm{H}), 1.75-1.65(\mathrm{~m}, 3 \mathrm{H}) \mathrm{ppm} .{ }^{13} \mathrm{C}$ NMR $\left(126 \mathrm{MHz}, \mathrm{CDCl}_{3}\right.$ ): $\delta$ 162.4, 134.0, 133.4, 132.4, 131.7, 128.3, 128.1, 127.8, 126.3 (q, $J_{C-F}=278.1$ $\mathrm{Hz}), 123.3,89.7,83.0,64.9,39.3\left(\mathrm{q}, J_{C-F}=27.7 \mathrm{~Hz}\right), 34.4,28.4,26.6,23.6$ ppm. ${ }^{19} \mathbf{F} \mathbf{N M R}(470 \mathrm{MHz}$, $\left.\mathrm{CDCl}_{3}\right): \delta-65.13(\mathrm{t}, J=10.6 \mathrm{~Hz}, 3 \mathrm{~F})$. HRMS m/z (ESI): calcd. for $\mathrm{C}_{20} \mathrm{H}_{19} \mathrm{~F}_{3} \mathrm{O}_{2} \mathrm{SNa}[\mathrm{M}+\mathrm{Na}]^{+}: 403.0950$; found: 403.0947 .<smiles>O=C(OCCCCC(C#CC(F)(F)F)CC(F)(F)F)c1ccccn1</smiles>

3o: 7-phenyl-5-(2,2,2-trifluoroethyl)hept-6-yn-1-yl 4-cyanobenzoate. Prepared according to the general procedure. Reaction was run using 10 (41 mg, $0.2 \mathrm{mmol})$, NaOAc (3.3 $\mathrm{mg}, 0.04 \mathrm{mmol})$, $\mathrm{AgOCOCF}_{3}$ (4.4 mg, $0.02 \mathrm{mmol}$ ), BIOMe (22.2 mg, $\left.0.08 \mathrm{mmol}\right), \mathrm{Me}_{3} \mathrm{SiCF}_{3}(120 \mu \mathrm{L}, 0.8 \mathrm{mmol}), 2 \mathrm{a}$ $(208.8 \mathrm{mg}, 0.6 \mathrm{mmol})$ and DMSO $(1.0 \mathrm{~mL})$. The product was purified by flash column chromatography on silica gel (hexane/EtOAc) and obtained a colorless oil $(0.16 \mathrm{mmol}, 60.0 \mathrm{mg}, 80 \%), \mathrm{R}_{\mathrm{f}}=0.30$ (hexane : EtOAc $=2: 1) .{ }^{1} \mathbf{H}$ NMR $\left(400 \mathrm{MHz}, \mathrm{CDCl}_{3}\right): \delta 8.77(\mathrm{~d}, J=4.8 \mathrm{~Hz}, 1 \mathrm{H}), 8.12(\mathrm{~d}, J=8.0 \mathrm{~Hz}, 1 \mathrm{H}), 7.79$ $(\mathrm{dt}, J=8.0 \mathrm{~Hz}, J=1.6 \mathrm{~Hz}, 1 \mathrm{H}), 7.48-7.45(\mathrm{~m}, 1 \mathrm{H}), 7.38-7.36(\mathrm{~m}, 2 \mathrm{H}), 7.30-7.26(\mathrm{~m}, 3 \mathrm{H}), 4.48(\mathrm{t}, J=$ $6.6 \mathrm{~Hz}, 2 \mathrm{H}), 3.00-2.94(\mathrm{~m}, 1 \mathrm{H}), 2.52-2.39(\mathrm{~m}, 1 \mathrm{H}), 2.37-2.26(\mathrm{~m}, 1 \mathrm{H}), 1.97-1.84(\mathrm{~m}, 2 \mathrm{H}), 1.82-1.64(\mathrm{~m}$, 4H) ppm. ${ }^{13} \mathbf{C}$ NMR (101 MHz, $\left.\mathrm{CDCl}_{3}\right): \delta 165.3,150.0,148.2,137.1,131.6,128.3,128.0,126.9,126.2$ $\left(\mathrm{q}, J_{C-F}=278.5 \mathrm{~Hz}\right), 125.2,123.2,89.7,82.9,65.7,39.2\left(\mathrm{q}, J_{C-F}=27.9 \mathrm{~Hz}\right), 34.4,28.3,26.5,23.5 \mathrm{ppm}$. ${ }^{19}$ F NMR $\left(470 \mathrm{MHz}, \mathrm{CDCl}_{3}\right): \delta-65.13(\mathrm{t}, J=10.6 \mathrm{~Hz}, 3 \mathrm{~F})$. HRMS m/z (ESI): calcd. for $\mathrm{C}_{21} \mathrm{H}_{20} \mathrm{~F}_{3} \mathrm{NO}_{2} \mathrm{Na}$ $[\mathrm{M}+\mathrm{Na}]^{+}:$398.1338; found: 398.1337 .<smiles>FC(F)(F)CC(C#CPc1ccccc1)CCCCOc1ccc(I)cc1</smiles>

3p: 1-iodo-4-((7-phenyl-5-(2,2,2-trifluoroethyl)hept-6-yn-1-yl)oxy)benzene. Prepared according to the general procedure. Reaction was run using 1p (60.4 mg, $0.2 \mathrm{mmol})$, NaOAc (3.3 $\mathrm{mg}, 0.04 \mathrm{mmol})$, $\mathrm{AgOCOCF}_{3}$ (4.4 mg, $0.02 \mathrm{mmol}$ ), BIOMe (22.2 mg, $\left.0.08 \mathrm{mmol}\right), \mathrm{Me}_{3} \mathrm{SiCF}_{3}(120 \mu \mathrm{L}, 0.8 \mathrm{mmol}), 2 \mathrm{a}$ (208.8 mg, $0.6 \mathrm{mmol})$ and DMSO $(1.0 \mathrm{~mL})$. The product was purified by flash column chromatography on silica gel (hexane/EtOAc) and obtained a colorless oil ( $0.12 \mathrm{mmol}, 56.6 \mathrm{mg}, 60 \%), \mathrm{R}_{\mathrm{f}}=0.30$ (hexane). ${ }^{1}$ H NMR (400 MHz, $\mathrm{CDCl}_{3}$ ): $\delta 7.55(\mathrm{~d}, J=8.8 \mathrm{~Hz}, 2 \mathrm{H}), 7.42-7.39(\mathrm{~m}, 2 \mathrm{H}), 7.32-7.30$ (m, 3H), $6.68(\mathrm{~d}$, $J=8.8 \mathrm{~Hz}, 2 \mathrm{H}), 3.96(\mathrm{t}, J=6.2 \mathrm{~Hz}, 2 \mathrm{H}), 3.02-2.95(\mathrm{~m}, 1 \mathrm{H}), 2.55-2.41(\mathrm{~m}, 1 \mathrm{H}), 2.38-2.25$ (m, 1H), $1.91-$ $1.78(\mathrm{~m}, 3 \mathrm{H}), 1.74-1.65$ (m, 3H) ppm. ${ }^{13} \mathbf{C}$ NMR (101 MHz, $\left.\mathrm{CDCl}_{3}\right): \delta 159.0,138.3,131.7,128.4,128.1$, $126.3\left(\mathrm{q}, J_{C-F}=278.6 \mathrm{~Hz}\right), 123.1,117.0,89.8,83.0,82.7,67.8,39.3\left(\mathrm{q}, J_{C-F}=27.8 \mathrm{~Hz}\right), 34.5,28.9,26.6$, 23.7 ppm. ${ }^{19}$ F NMR (470 MHz, $\left.\mathrm{CDCl}_{3}\right): \delta-65.17$ (q, $\left.J=10.7 \mathrm{~Hz}, 3 \mathrm{~F}\right)$. HRMS m/z (APCI): calcd. for $\mathrm{C}_{21} \mathrm{H}_{21} \mathrm{~F}_{3} \mathrm{IO}[\mathrm{M}+\mathrm{H}]^{+}$: 473.0584 ; found: 473.0581 . 


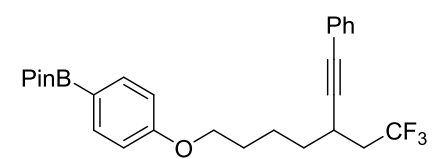

3q: 4,4,5,5-tetramethyl-2-(4-((7-phenyl-5-(2,2,2-trifluoroethyl)hept-6-yn-1-yl)oxy)phenyl)-1,3,2dioxaborolane. Prepared according to the general procedure. Reaction was run using $\mathbf{1 q}(60.4 \mathrm{mg}, 0.2$ mmol), NaOAc (3.3 mg, $0.04 \mathrm{mmol}), \mathrm{AgOCOCF}_{3}(4.4 \mathrm{mg}, 0.02 \mathrm{mmol}), \mathrm{BIOMe}(22.2 \mathrm{mg}, 0.08 \mathrm{mmol})$, $\mathrm{Me}_{3} \mathrm{SiCF}_{3}(120 \mu \mathrm{L}, 0.8 \mathrm{mmol}), \mathbf{2 a}(208.8 \mathrm{mg}, 0.6 \mathrm{mmol})$ and DMSO $(1.0 \mathrm{~mL})$. The product was purified by flash column chromatography on silica gel (hexane/EtOAc) and obtained a colorless oil (0.09 mmol, $42.5 \mathrm{mg}, 45 \%), \mathrm{R}_{\mathrm{f}}=0.5$ (hexane : EtOAc $\left.=8: 1\right) .{ }^{1} \mathbf{H} \mathbf{N M R}\left(500 \mathrm{MHz}, \mathrm{CDCl}_{3}\right): \delta 7.76(\mathrm{~d}, J=8.5 \mathrm{~Hz}$, 2H), 7.42-7.39 (m, 2H), 7.32-7.28 (m, 3H), $6.90(\mathrm{~d}, J=7.5 \mathrm{~Hz}, 2 \mathrm{H}), 4.03(\mathrm{t}, J=6.0 \mathrm{~Hz}, 2 \mathrm{H}), 3.01-2.96$ $(\mathrm{m}, 1 \mathrm{H}), 2.52-2.41(\mathrm{~m}, 1 \mathrm{H}), 2.36-2.26(\mathrm{~m}, 1 \mathrm{H}), 1.92-1.77(\mathrm{~m}, 3 \mathrm{H}), 1.75-1.64(\mathrm{~m}, 3 \mathrm{H}), 1.35(\mathrm{~s}, 12 \mathrm{H}) \mathrm{ppm}$. ${ }^{13} \mathrm{C}$ NMR (126 MHz, $\left.\mathrm{CDCl}_{3}\right): \delta 161.7,136.6,131.7,128.4,128.1,126.3\left(\mathrm{q}, J_{C-F}=278.2 \mathrm{~Hz}\right), 123.4$, 114.0, 89.9, 83.7, 83.0, 67.5, 39.3 (q, $\left.J_{C-F}=27.7 \mathrm{~Hz}\right), 34.6,28.9,26.6,25.0,23.7$ ppm. ${ }^{19} \mathbf{F}$ NMR $(470$ $\left.\mathrm{MHz}, \mathrm{CDCl}_{3}\right): \delta-65.08(\mathrm{t}, J=10.6 \mathrm{~Hz}, 3 \mathrm{~F})$. HRMS m/z (ESI): calcd. for $\mathrm{C}_{27} \mathrm{H}_{32} \mathrm{BF}_{3} \mathrm{O}_{3} \mathrm{Na}[\mathrm{M}+\mathrm{Na}]^{+}$: 495.2294; found: 495.2292.<smiles>CC(=O)c1ccc(OCCCCC(C#Cc2ccccc2)CC(F)(F)F)cc1</smiles>

3r: 1-(4-((7-phenyl-5-(2,2,2-trifluoroethyl)hept-6-yn-1-yl)oxy)phenyl)ethan-1-one. Prepared according to the general procedure. Reaction was run using $1 \mathbf{r}(43.6 \mathrm{mg}, 0.2 \mathrm{mmol}), \mathrm{NaOAc}(3.3 \mathrm{mg}$, $0.04 \mathrm{mmol}$ ), $\mathrm{AgOCOCF}_{3}(4.4 \mathrm{mg}, 0.02 \mathrm{mmol}), \mathrm{BIOMe}(22.2 \mathrm{mg}, 0.08 \mathrm{mmol}), \mathrm{Me}_{3} \mathrm{SiCF}_{3}(120 \mu \mathrm{L}, 0.8$ $\mathrm{mmol})$, $2 \mathrm{a}(208.8 \mathrm{mg}, 0.6 \mathrm{mmol})$ and DMSO $(1.0 \mathrm{~mL})$. The product was purified by flash column chromatography on silica gel (hexane/EtOAc) and obtained a colorless oil (0.16 mmol, $63.6 \mathrm{mg}, 82 \%)$, $\mathrm{R}_{\mathrm{f}}=0.4$ (hexane : EtOAc $\left.=5: 1\right) .{ }^{1} \mathbf{H} \mathbf{N M R}\left(500 \mathrm{MHz}, \mathrm{CDCl}_{3}\right): \delta 7.92(\mathrm{~d}, J=9.0 \mathrm{~Hz}, 2 \mathrm{H}), 7.40-7.38(\mathrm{~m}$, 2H), 7.30-7.28 (m, 3H), $6.92(\mathrm{~d}, J=9.0 \mathrm{~Hz}, 2 \mathrm{H}), 4.06(\mathrm{t}, J=6.3 \mathrm{~Hz}, 2 \mathrm{H}), 3.01-2.95(\mathrm{~m}, 1 \mathrm{H}), 2.55$ (s, $3 \mathrm{H}), 2.53-2.42(\mathrm{~m}, 1 \mathrm{H}), 2.37-2.26(\mathrm{~m}, 1 \mathrm{H}), 1.95-1.79(\mathrm{~m}, 3 \mathrm{H}), 1.76-1.65(\mathrm{~m}, 3 \mathrm{H}) \mathrm{ppm} .{ }^{13} \mathbf{C} \mathbf{N M R}(126$ $\left.\mathrm{MHz}_{1} \mathrm{CDCl}_{3}\right): \delta 196.9,163.1,131.7,130.7,130.3,128.4,128.1,126.3\left(\mathrm{q}, J_{C-F}=278.1 \mathrm{~Hz}\right), 123.3,114.2$, 89.8, 83.0, 68.0, 39.2 (q, $\left.J_{C-F}=27.7 \mathrm{~Hz}\right), 34.5,28.8,26.6,26.4,23.7$ ppm. ${ }^{19} \mathbf{F} \mathbf{N M R}\left(470 \mathrm{MHz}, \mathrm{CDCl}_{3}\right)$ : $\delta-65.13(\mathrm{t}, J=10.6 \mathrm{~Hz}, 3 \mathrm{~F})$. HRMS m/z (ESI): calcd. for $\mathrm{C}_{23} \mathrm{H}_{23} \mathrm{~F}_{3} \mathrm{O}_{2} \mathrm{Na}[\mathrm{M}+\mathrm{Na}]^{+}: 411.1542$; found: 411.1542 .<smiles>COC(c1ccc(OCCCCC(C#CC(CF)CF)CC(F)(F)F)cc1)C(F)(F)F</smiles>

3s: 4-((7-phenyl-5-(2,2,2-trifluoroethyl)hept-6-yn-1-yl)oxy)benzaldehyde and 3s': trimethyl(2,2,2trifluoro-1-(4-((7-phenyl-5-(2,2,2-trifluoroethyl)hept-6-yn-1-yl)oxy)phenyl)ethoxy)silane. Prepared according to the general procedure. Reaction was run using $1 \mathrm{~s}$ (40.8 $\mathrm{mg}, 0.2 \mathrm{mmol}), \mathrm{NaOAc}(3.3 \mathrm{mg}$, $0.04 \mathrm{mmol}), \mathrm{AgOCOCF}_{3}(4.4 \mathrm{mg}, 0.02 \mathrm{mmol}), \mathrm{BIOMe}(22.2 \mathrm{mg}, 0.08 \mathrm{mmol}), \mathrm{Me}_{3} \mathrm{SiCF}_{3}(120 \mu \mathrm{L}, 0.8$ $\mathrm{mmol})$, $2 \mathrm{a}(208.8 \mathrm{mg}, 0.6 \mathrm{mmol})$ and DMSO $(1.0 \mathrm{~mL})$. The product was purified by flash column chromatography on silica gel (hexane/EtOAc) and obtained $3 \mathrm{~s}$ [0.07 mmol, $26.1 \mathrm{mg}, 35 \%, \mathrm{R}_{\mathrm{f}}=0.8$ (hexane : EtOAc $=5: 1$ ) $]$ and 3s' $\left[0.07 \mathrm{mmol}, 38.2 \mathrm{mg}, 37 \%, \mathrm{R}_{\mathrm{f}}=0.4\right.$ (hexane : EtOAc $=5: 1$ ) $]$ as a colorless oil. 
3s: ${ }^{1} \mathrm{H}$ NMR (500 MHz, $\left.\mathrm{CDCl}_{3}\right): \delta 9.88(\mathrm{~s}, 1 \mathrm{H}), 7.82(\mathrm{~d}, J=8.5 \mathrm{~Hz}, 2 \mathrm{H}), 7.40-7.38(\mathrm{~m}, 2 \mathrm{H}), 7.30-7.28$ (m, 3H), $6.99(\mathrm{~d}, J=8.5 \mathrm{~Hz}, 2 \mathrm{H}), 4.08(\mathrm{t}, J=6.3 \mathrm{~Hz}, 2 \mathrm{H}), 3.00-2.94(\mathrm{~m}, 1 \mathrm{H}), 2.51-2.40(\mathrm{~m}, 1 \mathrm{H}), 2.36-$ $2.28(\mathrm{~m}, 1 \mathrm{H}), 1.96-1.80(\mathrm{~m}, 3 \mathrm{H}), 1.77-1.65(\mathrm{~m}, 3 \mathrm{H}) \mathrm{ppm} .{ }^{13} \mathbf{C} \mathbf{N M R}\left(126 \mathrm{MHz}, \mathrm{CDCl}_{3}\right): \delta 190.9,164.2$, $132.1,131.7,130.3,128.4,128.2,126.3\left(\mathrm{q}, J_{C-F}=278.1 \mathrm{~Hz}\right), 123.3,114.9,89.8,83.0,68.1,39.3\left(\mathrm{q}, J_{C-}\right.$ $\left.{ }_{F}=27.7 \mathrm{~Hz}\right), 34.5,28.8,26.6,23.7 \mathrm{ppm} .{ }^{19} \mathbf{F}$ NMR $\left(470 \mathrm{MHz}, \mathrm{CDCl}_{3}\right): \delta-65.14(\mathrm{t}, J=10.6 \mathrm{~Hz}, 3 \mathrm{~F})$. HRMS m/z (ESI): calcd. for $\mathrm{C}_{22} \mathrm{H}_{21} \mathrm{~F}_{3} \mathrm{O}_{2} \mathrm{Na}[\mathrm{M}+\mathrm{Na}]^{+}: 397.1386$; found: 397.1387 .

3s': ' ${ }^{1} \mathbf{H}$ NMR (500 MHz, $\left.\mathrm{CDCl}_{3}\right):$ 87.41-7.39 (m, 2H), 7.35 (d, $\left.J=8.5 \mathrm{~Hz}, 2 \mathrm{H}\right), 7.31-7.29$ (m, 3H), 6. 89 (d, $J=9.0 \mathrm{~Hz}, 2 \mathrm{H}), 4.86(\mathrm{q}, J=6.5 \mathrm{~Hz}, 1 \mathrm{H}), 4.01(\mathrm{t}, J=6.0 \mathrm{~Hz}, 2 \mathrm{H}), 3.00-2.95(\mathrm{~m}, 1 \mathrm{H}), 2.52-2.41$ $(\mathrm{m}, 1 \mathrm{H}), 2.37-2.26(\mathrm{~m}, 1 \mathrm{H}), 1.92-1.78(\mathrm{~m}, 3 \mathrm{H}), 1.77-1.65(\mathrm{~m}, 3 \mathrm{H}), 0.11(\mathrm{~s}, 9 \mathrm{H}) \mathrm{ppm} .{ }^{13} \mathbf{C}$ NMR $(126$ $\left.\mathrm{MHz}, \mathrm{CDCl}_{3}\right): \delta 159.7,131.8,128.9,128.4,128.1,127.6,126.3\left(\mathrm{q}, J_{C-F}=278.0 \mathrm{~Hz}\right), 124.4\left(\mathrm{q}, J_{C-F}=\right.$ $282.7 \mathrm{~Hz}), 123.4,114.3,89.9,83.0,73.0\left(\mathrm{q}, J_{C-F}=32.3 \mathrm{~Hz}\right), 67.8,39.3$ (q, $\left.J_{C-F}=27.7 \mathrm{~Hz}\right), 34.6,29.0$, 26.6, 23.8, -0.12 ppm. ${ }^{19} \mathbf{F}$ NMR (470 MHz, $\left.\mathrm{CDCl}_{3}\right): \delta-65.14(\mathrm{t}, J=10.8 \mathrm{~Hz}, 3 \mathrm{~F}),-79.73(\mathrm{~d}, J=6.6 \mathrm{~Hz}$, 3F). HRMS m/z (APCI): calcd. for $\mathrm{C}_{26} \mathrm{H}_{31} \mathrm{~F}_{6} \mathrm{O}_{2} \mathrm{Si}[\mathrm{M}+\mathrm{H}]^{+}$: 517.1992; found: 517.1992.

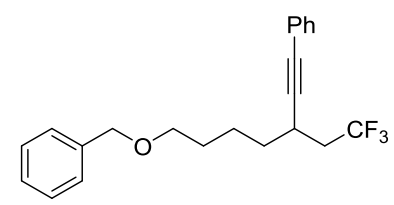

3t: (7-(benzyloxy)-3-(2,2,2-trifluoroethyl)hept-1-yn-1-yl)benzene. Prepared according to the general procedure. Reaction was run using $1 \mathbf{t}$ (38.0 mg, $0.2 \mathrm{mmol}$ ), $\mathrm{NaOAc}(3.3 \mathrm{mg}, 0.04 \mathrm{mmol}), \mathrm{AgOCOCF}_{3}$ (4.4 mg, $0.02 \mathrm{mmol}), \operatorname{BIOMe}(22.2 \mathrm{mg}, 0.08 \mathrm{mmol}), \mathrm{Me}_{3} \mathrm{SiCF}_{3}(120 \mu \mathrm{L}, 0.8 \mathrm{mmol}), 2 \mathrm{a}(208.8 \mathrm{mg}, 0.6$ $\mathrm{mmol})$ and DMSO $(1.0 \mathrm{~mL})$. The product was purified by flash column chromatography on silica gel (hexane/EtOAc) and obtained a colorless oil $\left(0.15 \mathrm{mmol}, 54.7 \mathrm{mg}, 76 \%\right.$ ), $\mathrm{R}_{\mathrm{f}}=0.4$ (hexane : EtOAc $=5$ : 1). ${ }^{1} \mathbf{H}$ NMR (400 MHz, $\left.\mathrm{CDCl}_{3}\right): \delta$ 7.43-7.40 (m, 2H), 7.38-7.34 (m, 4H), 7.33-7.28 (m, 4H), $4.53(\mathrm{~s}$, 2H), $3.53(\mathrm{t}, J=6.0 \mathrm{~Hz}, 2 \mathrm{H}), 2.99-2.92(\mathrm{~m}, 1 \mathrm{H}), 2.52-2.39(\mathrm{~m}, 1 \mathrm{H}), 2.36-2.24(\mathrm{~m}, 1 \mathrm{H}), 1.77-1.59(\mathrm{~m}$, 6H) ppm. ${ }^{13} \mathbf{C}$ NMR $\left(101 \mathrm{MHz}, \mathrm{CDCl}_{3}\right): \delta 138.7,131.7,128.5,128.3,128.1,127.8,127.6,126.4\left(\mathrm{q}, J_{C-}\right.$ $\left.{ }_{F}=278.5 \mathrm{~Hz}\right), 123.4,90.1,82.8,73.0,70.2,39.3\left(\mathrm{q}, J_{C-F}=27.8 \mathrm{~Hz}\right), 34.7,29.5,26.6,23.8 \mathrm{ppm} .{ }^{19} \mathbf{F}$ NMR (470 MHz, $\left.\mathrm{CDCl}_{3}\right): \delta-65.12(\mathrm{t}, J=10.6 \mathrm{~Hz}, 3 \mathrm{~F})$. HRMS m/z (ESI): calcd. for $\mathrm{C}_{22} \mathrm{H}_{23} \mathrm{~F}_{3} \mathrm{ONa}$ $[\mathrm{M}+\mathrm{Na}]^{+}: 383.1593$; found: 383.1591 .<smiles>CCCCC(C#Cc1ccccc1)CCCOC</smiles>

3u: tert-butyldimethyl((7-phenyl-5-(2,2,2-trifluoroethyl)hept-6-yn-1-yl)oxy)silane. Prepared according to the general procedure. Reaction was run using $1 \mathbf{u}(42.8 \mathrm{mg}, 0.2 \mathrm{mmol}), \mathrm{NaOAc}(3.3 \mathrm{mg}$, $0.04 \mathrm{mmol}$ ), $\mathrm{AgOCOCF}_{3}(4.4 \mathrm{mg}, 0.02 \mathrm{mmol}$ ), BIOMe (22.2 mg, $0.08 \mathrm{mmol}), \mathrm{Me}_{3} \mathrm{SiCF}_{3}(120 \mu \mathrm{L}, 0.8$ $\mathrm{mmol}), 2 \mathrm{a}(208.8 \mathrm{mg}, 0.6 \mathrm{mmol})$ and DMSO $(1.0 \mathrm{~mL})$. The product was purified by flash column chromatography on silica gel (hexane/EtOAc) and obtained a colorless oil ( $0.16 \mathrm{mmol}, 60.0 \mathrm{mg}, 78 \%$ ), $\mathrm{R}_{\mathrm{f}}=0.2$ (hexane). ${ }^{1} \mathbf{H}$ NMR $\left(500 \mathrm{MHz}, \mathrm{CDCl}_{3}\right): \delta$ 7.42-7.39 (m, 2H), 7.31-7.28 (m, 3H), $3.66(\mathrm{t}, J=6.0$ $\mathrm{Hz}, 2 \mathrm{H}), 2.98-2.92(\mathrm{~m}, 1 \mathrm{H}), 2.49-2.39(\mathrm{~m}, 1 \mathrm{H}), 2.35-2.25(\mathrm{~m}, 1 \mathrm{H}), 1.70-1.53(\mathrm{~m}, 6 \mathrm{H}), 0.91(\mathrm{~s}, 9 \mathrm{H}), 0.07$ (s, 6H) ppm. ${ }^{13} \mathbf{C}$ NMR (126 MHz, $\mathrm{CDCl}_{3}$ ): $\delta 131.7,128.3,128.1,126.4$ (q, $\left.J_{C-F}=278.0 \mathrm{~Hz}\right), 123.5,90.1$, $82.8,63.1,39.3\left(\mathrm{q}, J_{C-F}=27.7 \mathrm{~Hz}\right), 34.8,32.5,26.6,26.1,23.5,18.5,-5.2 \mathrm{ppm} .{ }^{19} \mathbf{F}$ NMR $(470 \mathrm{MHz}$, $\mathrm{CDCl}_{3}$ ): $\delta-65.15$ (t, $J=10.6 \mathrm{~Hz}, 3 \mathrm{~F}$ ). HRMS m/z (APCI): calcd. for $\mathrm{C}_{21} \mathrm{H}_{32} \mathrm{~F}_{3} \mathrm{OSi}[\mathrm{M}+\mathrm{H}]^{+}: 385.2169$; found: 385.2166 . 


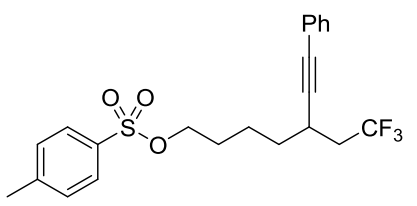

3v: 7-phenyl-5-(2,2,2-trifluoroethyl)hept-6-yn-1-yl 4-methylbenzenesulfonate. Prepared according to the general procedure. Reaction was run using $1 \mathbf{v}(50.8 \mathrm{mg}, 0.2 \mathrm{mmol}), \mathrm{NaOAc}(3.3 \mathrm{mg}, 0.04 \mathrm{mmol})$, $\mathrm{AgOCOCF}_{3}$ (4.4 mg, $0.02 \mathrm{mmol}$ ), BIOMe (22.2 mg, $\left.0.08 \mathrm{mmol}\right), \mathrm{Me}_{3} \mathrm{SiCF}_{3}(120 \mu \mathrm{L}, 0.8 \mathrm{mmol}), 2 \mathrm{a}$ $(208.8 \mathrm{mg}, 0.6 \mathrm{mmol})$ and DMSO $(1.0 \mathrm{~mL})$. The product was purified by flash column chromatography on silica gel (hexane/EtOAc) and obtained a colorless oil $(0.08 \mathrm{mmol}, 33.9 \mathrm{mg}, 40 \%), \mathrm{R}_{\mathrm{f}}=0.2$ (hexane : EtOAc = $8: 1)$. ${ }^{1} \mathbf{H}$ NMR $\left(400 \mathrm{MHz}, \mathrm{CDCl}_{3}\right): \delta 7.79(\mathrm{~d}, J=8.4 \mathrm{~Hz}, 2 \mathrm{H}), 7.40-7.37(\mathrm{~m}, 2 \mathrm{H}), 7.32-7.26$ $(\mathrm{m}, 5 \mathrm{H}), 4.06(\mathrm{t}, J=6.2 \mathrm{~Hz}, 2 \mathrm{H}), 2.90-2.84(\mathrm{~m}, 1 \mathrm{H}), 2.47-2.35(\mathrm{~m}, 1 \mathrm{H}), 2.42(\mathrm{~s}, 3 \mathrm{H}), 2.30-2.17(\mathrm{~m}, 1 \mathrm{H})$, 1.76-1.46 (m, 6H) ppm. ${ }^{13} \mathbf{C}$ NMR (101 MHz, $\left.\mathrm{CDCl}_{3}\right): \delta 144.9,133.2,131.7,130.0,128.4,128.2,128.0$, $126.2\left(\mathrm{q}, J_{C-F}=278.6 \mathrm{~Hz}\right), 123.2,89.5,83.0,70.3,39.2\left(\mathrm{q}, J_{C-F}=27.9 \mathrm{~Hz}\right), 34.1,28.5,26.5,23.1,21.7$ ppm. ${ }^{19} \mathbf{F}$ NMR $\left(470 \mathrm{MHz}, \mathrm{CDCl}_{3}\right): \delta-65.12$ (t, $\left.J=10.3 \mathrm{~Hz}, 3 \mathrm{~F}\right)$. HRMS m/z (ESI): calcd. for $\mathrm{C}_{22} \mathrm{H}_{23} \mathrm{~F}_{3} \mathrm{O}_{3} \mathrm{SNa}[\mathrm{M}+\mathrm{Na}]^{+}: 447.1212$; found: 447.1210.<smiles>O=C(CCCCCBr)OCCCCC(C#Cc1ccccc1)CC(F)(F)F</smiles>

3w: 7-phenyl-5-(2,2,2-trifluoroethyl)hept-6-yn-1-yl 6-bromohexanoate. Prepared according to the general procedure. Reaction was run using $1 \mathbf{w}(55.2 \mathrm{mg}, 0.2 \mathrm{mmol}), \mathrm{NaOAc}(3.3 \mathrm{mg}, 0.04 \mathrm{mmol})$, $\mathrm{AgOCOCF}_{3}(4.4 \mathrm{mg}, 0.02 \mathrm{mmol}), \mathrm{BIOMe}(22.2 \mathrm{mg}, 0.08 \mathrm{mmol}), \mathrm{Me}_{3} \mathrm{SiCF}_{3}(120 \mu \mathrm{L}, 0.8 \mathrm{mmol}), 2 \mathrm{a}$ $(208.8 \mathrm{mg}, 0.6 \mathrm{mmol})$ and DMSO $(1.0 \mathrm{~mL})$. The product was purified by flash column chromatography on silica gel (hexane/EtOAc) and obtained a colorless oil $(0.12 \mathrm{mmol}, 54.4 \mathrm{mg}, 61 \%), \mathrm{R}_{\mathrm{f}}=0.4$ (hexane : EtOAc $=8: 1) .{ }^{1} \mathbf{H}$ NMR $\left(500 \mathrm{MHz}, \mathrm{CDCl}_{3}\right): \delta 7.40-7.38(\mathrm{~m}, 2 \mathrm{H}), 7.30-7.26(\mathrm{~m}, 3 \mathrm{H}), 4.11(\mathrm{t}, J=6.0$ $\mathrm{Hz}, 2 \mathrm{H}), 3.38(\mathrm{t}, J=6.8 \mathrm{~Hz}, 2 \mathrm{H}), 2.96-2.91(\mathrm{~m}, 1 \mathrm{H}), 2.51-2.39(\mathrm{~m}, 1 \mathrm{H}), 2.34-2.24(\mathrm{~m}, 1 \mathrm{H}), 2.31(\mathrm{t}, J=$ $7.5 \mathrm{~Hz}, 2 \mathrm{H}), 1.87-1.81(\mathrm{~m}, 2 \mathrm{H}), 1.75-1.54(\mathrm{~m}, 8 \mathrm{H}), 1.47-1.41(\mathrm{~m}, 2 \mathrm{H}) \mathrm{ppm} .{ }^{13} \mathbf{C} \mathbf{N M R}\left(126 \mathrm{MHz}, \mathrm{CDCl}_{3}\right)$ $\delta 173.6,131.7,128.3,128.1,126.3\left(\mathrm{q}, J_{C-F}=278.0 \mathrm{~Hz}\right), 123.3,89.7,82.9,64.2,39.2\left(\mathrm{q}, J_{C-F}=27.7 \mathrm{~Hz}\right)$, 34.4, 34.1, 33.6, 32.4, 28.3, 27.7, 26.5, 24.3, 23.6 ppm. ${ }^{19} \mathbf{F}$ NMR $\left(470 \mathrm{MHz}, \mathrm{CDCl}_{3}\right): \delta-65.12(\mathrm{t}, J=$ $10.8 \mathrm{~Hz}, 3 \mathrm{~F})$. HRMS m/z (ESI): calcd. for $\mathrm{C}_{21} \mathrm{H}_{26} \mathrm{BrF}_{3} \mathrm{O}_{2} \mathrm{Na}[\mathrm{M}+\mathrm{Na}]^{+}$: 469.0961 ; found: 469.0962 .<smiles>COC(=O)CCCCCCCCC(C#CCC(F)(F)F)CC(F)(F)F</smiles>

3x: methyl 12-phenyl-10-(2,2,2-trifluoroethyl)dodec-11-ynoate. Prepared according to the general procedure. Reaction was run using $1 \times 2$ (39.6 mg, $0.2 \mathrm{mmol})$, NaOAc (3.3 mg, $0.04 \mathrm{mmol}), \mathrm{AgOCOCF}_{3}$ (4.4 mg, $0.02 \mathrm{mmol})$, BIOMe (22.2 mg, $0.08 \mathrm{mmol}), \mathrm{Me}_{3} \mathrm{SiCF}_{3}(120 \mu \mathrm{L}, 0.8 \mathrm{mmol}), 2 \mathrm{a}(208.8 \mathrm{mg}, 0.6$ $\mathrm{mmol})$ and DMSO $(1.0 \mathrm{~mL})$. The product was purified by flash column chromatography on silica gel (hexane/EtOAc) and obtained a colorless oil $(0.15 \mathrm{mmol}, 55.9 \mathrm{mg}, 76 \%), \mathrm{R}_{\mathrm{f}}=0.6$ (hexane : EtOAc $=8$ : 1). ${ }^{1} \mathbf{H}$ NMR $\left(500 \mathrm{MHz}, \mathrm{CDCl}_{3}\right): \delta$ 7.41-7.39 (m, 2H), 7.30-7.26 (m, 3H), $3.66(\mathrm{~s}, 3 \mathrm{H}), 2.95-2.90(\mathrm{~m}$, $1 \mathrm{H}), 2.48-2.37(\mathrm{~m}, 1 \mathrm{H}), 2.34-2.24(\mathrm{~m}, 1 \mathrm{H}), 2.30(\mathrm{t}, J=7.5 \mathrm{~Hz}, 2 \mathrm{H}), 1.65-1.55(\mathrm{~m}, 5 \mathrm{H}), 1.51-1.44(\mathrm{~m}$, $1 \mathrm{H}), 1.37-1.28(\mathrm{~m}, 8 \mathrm{H})$ ppm. ${ }^{13} \mathbf{C}$ NMR (101 MHz, $\left.\mathrm{CDCl}_{3}\right): \delta 174.4,131.7,128.3,128.0,126.3\left(\mathrm{q}, J_{C-F}\right.$ $=278.5 \mathrm{~Hz}), 123.5,90.3,82.6,51.6,39.2$ (q, $\left.J_{C-F}=27.8 \mathrm{~Hz}\right), 34.9,34.2,29.4,29.3,29.3,29.2,27.0$, 
26.6, 25.0 ppm. ${ }^{19}$ F NMR (470 MHz, $\left.\mathrm{CDCl}_{3}\right): \delta-65.16$ (t, $\left.J=10.8 \mathrm{~Hz}, 3 \mathrm{~F}\right) . \mathbf{H R M S ~ m} / \mathrm{z}$ (ESI): calcd. for $\mathrm{C}_{21} \mathrm{H}_{27} \mathrm{~F}_{3} \mathrm{O}_{2} \mathrm{Na}[\mathrm{M}+\mathrm{Na}]^{+}$: 391.1855; found: 391.1854 .<smiles>O=C(CCCCCCCCCC(C#CCC(F)(F)F)Cc1ccccc1)N1CCOCC1</smiles>

3y: 1-morpholino-12-phenyl-10-(2,2,2-trifluoroethyl)dodec-11-yn-1-one. Prepared according to the general procedure. Reaction was run using 1 y $(50.6 \mathrm{mg}, 0.2 \mathrm{mmol}), \mathrm{NaOAc}(3.3 \mathrm{mg}, 0.04 \mathrm{mmol})$, $\mathrm{AgOCOCF}_{3}$ (4.4 mg, $0.02 \mathrm{mmol}$ ), BIOMe (22.2 mg, $\left.0.08 \mathrm{mmol}\right), \mathrm{Me}_{3} \mathrm{SiCF}_{3}(120 \mu \mathrm{L}, 0.8 \mathrm{mmol}), 2 \mathrm{a}$ $(208.8 \mathrm{mg}, 0.6 \mathrm{mmol})$ and DMSO $(1.0 \mathrm{~mL})$. The product was purified by flash column chromatography on silica gel (hexane/EtOAc) and obtained a colorless oil $(0.17 \mathrm{mmol}, 70.2 \mathrm{mg}, 83 \%), \mathrm{R}_{\mathrm{f}}=0.3$ (hexane : EtOAc $=2: 1) .{ }^{1} \mathbf{H}$ NMR $\left(400 \mathrm{MHz}, \mathrm{CDCl}_{3}\right): \delta$ 7.42-7.40 (m, 2H), 7.31-7.28 (m, 3H), $3.66(\mathrm{~m}, 4 \mathrm{H})$, $3.63(\mathrm{~m}, 2 \mathrm{H}), 3.45(\mathrm{~m}, 2 \mathrm{H}), 2.96-2.89(\mathrm{~m}, 1 \mathrm{H}), 2.50-2.38(\mathrm{~m}, 1 \mathrm{H}), 2.35-2.23(\mathrm{~m}, 1 \mathrm{H}), 2.30(\mathrm{t}, J=7.6 \mathrm{~Hz}$, $2 \mathrm{H}), 1.67-1.55(\mathrm{~m}, 5 \mathrm{H}), 1.52-1.45(\mathrm{~m}, 1 \mathrm{H}), 1.40-1.29(\mathrm{~m}, 8 \mathrm{H}) \mathrm{ppm} .{ }^{13} \mathbf{C} \mathbf{N M R}\left(126 \mathrm{MHz}, \mathrm{CDCl}_{3}\right): \delta$ $171.9,131.6,128.2,128.0,126.3\left(\mathrm{q}, J_{C-F}=278.1 \mathrm{~Hz}\right), 123.4,90.2,82.5,67.0,66.7,46.0,41.9,39.2(\mathrm{q}$, $\left.J_{C-F}=27.7 \mathrm{~Hz}\right), 34.8,33.1,29.4,29.4,29.3,29.2,26.9,26.5$ (q, $\left.J_{C-F}=2.9 \mathrm{~Hz}\right), 25.2$ ppm. ${ }^{19} \mathbf{F}$ NMR $(470$ $\left.\mathrm{MHz}_{\mathrm{CDCl}}\right): \delta-65.15(\mathrm{t}, J=10.8 \mathrm{~Hz}, 3 \mathrm{~F})$. HRMS m/z (ESI): calcd. for $\mathrm{C}_{24} \mathrm{H}_{32} \mathrm{~F}_{3} \mathrm{NO}_{2} \mathrm{Na}[\mathrm{M}+\mathrm{Na}]^{+}$: 446.2277; found: 446.2277 .

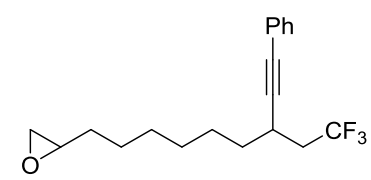

3z: 2-(9-phenyl-7-(2,2,2-trifluoroethyl)non-8-yn-1-yl)oxirane. Prepared according to the general procedure. Reaction was run using $\mathbf{1 z}(30.8 \mathrm{mg}, 0.2 \mathrm{mmol}), \mathrm{NaOAc}(3.3 \mathrm{mg}, 0.04 \mathrm{mmol}), \mathrm{AgOCOCF}_{3}$ (4.4 mg, $0.02 \mathrm{mmol}), \mathrm{BIOMe}(22.2 \mathrm{mg}, 0.08 \mathrm{mmol}), \mathrm{Me}_{3} \mathrm{SiCF}_{3}(120 \mu \mathrm{L}, 0.8 \mathrm{mmol}), 2 \mathrm{a}(208.8 \mathrm{mg}, 0.6$ mmol) and DMSO (1.0 mL). The product was purified by flash column chromatography on silica gel (hexane/EtOAc) and obtained a colorless oil $(0.13 \mathrm{mmol}, 42.1 \mathrm{mg}, 65 \%), \mathrm{R}_{\mathrm{f}}=0.4$ (hexane : EtOAc $=$ 20 : 1). ${ }^{1} \mathbf{H}$ NMR (500 MHz, $\left.\mathrm{CDCl}_{3}\right): \delta$ 7.41-7.39 (m, 2H), 7.30-7.28 (m, 3H), 2.95-2.86 (m, 2H), 2.74 $(\mathrm{t}, J=4.5 \mathrm{~Hz}, 1 \mathrm{H}), 2.49-2.38(\mathrm{~m}, 1 \mathrm{H}), 2.47-2.45(\mathrm{~m}, 1 \mathrm{H}), 2.34-2.23(\mathrm{~m}, 1 \mathrm{H}), 1.68-1.57(\mathrm{~m}, 3 \mathrm{H}), 1.57-$ $1.43(\mathrm{~m}, 5 \mathrm{H}), 1.43-1.32(\mathrm{~m}, 4 \mathrm{H}) \mathrm{ppm} .{ }^{13} \mathbf{C} \mathbf{N M R}\left(101 \mathrm{MHz}, \mathrm{CDCl}_{3}\right): \delta 131.7,128.3,128.0,126.4\left(\mathrm{q}, J_{C-}\right.$ $\left.{ }_{F}=278.6 \mathrm{~Hz}\right), 123.5,90.2,82.7,52.4,47.2,39.2\left(\mathrm{q}, J_{C-F}=27.7 \mathrm{~Hz}\right), 34.8,32.5,29.4,29.2,26.9,26.5$, 26.0 ppm. ${ }^{19} \mathbf{F}$ NMR $\left(470 \mathrm{MHz}, \mathrm{CDCl}_{3}\right): \delta-65.16(\mathrm{t}, J=10.6 \mathrm{~Hz}, 3 \mathrm{~F})$. HRMS m/z (ESI): calcd. for $\mathrm{C}_{19} \mathrm{H}_{23} \mathrm{~F}_{3} \mathrm{ONa}[\mathrm{M}+\mathrm{Na}]^{+}: 347.1593$; found: 347.1593 .<smiles></smiles>

3aa: 12-phenyl-10-(2,2,2-trifluoroethyl)dodec-11-ynoic acid. Prepared according to the general procedure. Reaction was run using 1 aa $(36.8 \mathrm{mg}, 0.2 \mathrm{mmol}), \mathrm{NaOAc}(3.3 \mathrm{mg}, 0.04 \mathrm{mmol}), \mathrm{AgOCOCF}_{3}$ (4.4 mg, $0.02 \mathrm{mmol}), \mathrm{BIOMe}(22.2 \mathrm{mg}, 0.08 \mathrm{mmol}), \mathrm{Me}_{3} \mathrm{SiCF}_{3}(120 \mu \mathrm{L}, 0.8 \mathrm{mmol}), 2 \mathrm{a}(208.8 \mathrm{mg}, 0.6$ $\mathrm{mmol})$ and DMSO $(1.0 \mathrm{~mL})$. The product was purified by flash column chromatography on silica gel 
(hexane/EtOAc) and obtained a colorless oil $(0.12 \mathrm{mmol}, 43.9 \mathrm{mg}, 62 \%), \mathrm{R}_{\mathrm{f}}=0.3$ (hexane : EtOAc $=3$ : 2). ${ }^{1} \mathbf{H}$ NMR (500 MHz, $\mathrm{CDCl}_{3}$ ): $\delta$ 7.41-7.39 (m, 2H), 7.30-7.27 (m, 3H), 2.95-2.90 (m, 1H), 2.48-2.38 (m, $1 \mathrm{H}), 2.35(\mathrm{t}, J=7.5 \mathrm{~Hz}, 2 \mathrm{H}), 2.35-2.24(\mathrm{~m}, 1 \mathrm{H}), 1.67-1.56(\mathrm{~m}, 5 \mathrm{H}), 1.52-1.44(\mathrm{~m}, 1 \mathrm{H}), 1.40-1.29$ (m, 8H) ppm. ${ }^{13} \mathbf{C}$ NMR (101 MHz, $\left.\mathrm{CDCl}_{3}\right): \delta 180.4,131.7,128.4,128.0,126.4$ (q, $\left.J_{C-F}=278.5 \mathrm{~Hz}\right)$, 123.5, 90.3, 82.7, 39.3 (q, $J_{C-F}=27.7 \mathrm{~Hz}$ ), 34.9, 34.2, 29.4, 29.3, 29.3, 29.1, 27.0, 26.6, 24.8 ppm. ${ }^{19} \mathbf{F}$ NMR (470 MHz, $\left.\mathrm{CDCl}_{3}\right): \delta-65.16(\mathrm{t}, J=10.6 \mathrm{~Hz}, 3 \mathrm{~F})$. HRMS m/z (ESI): calcd. for $\mathrm{C}_{20} \mathrm{H}_{25} \mathrm{~F}_{3} \mathrm{O}_{2} \mathrm{Na}$ $[\mathrm{M}+\mathrm{Na}]^{+}:$377.1699; found: 377.1698 .

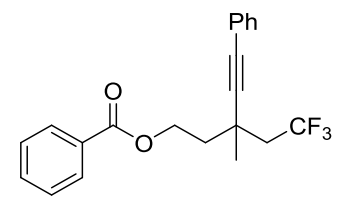

3ab: 3-methyl-5-phenyl-3-(2,2,2-trifluoroethyl)pent-4-yn-1-yl benzoate. Prepared according to the general procedure. Reaction was run using $1 \mathrm{ab}(38.0 \mathrm{mg}, 0.2 \mathrm{mmol}), \mathrm{NaOAc}(3.3 \mathrm{mg}, 0.04 \mathrm{mmol})$, $\mathrm{AgOCOCF}_{3}(4.4 \mathrm{mg}, 0.02 \mathrm{mmol})$, BIOMe (22.2 mg, $\left.0.08 \mathrm{mmol}\right), \mathrm{Me}_{3} \mathrm{SiCF}_{3}(120 \mu \mathrm{L}, 0.8 \mathrm{mmol}), 2 \mathrm{a}$ $(208.8 \mathrm{mg}, 0.6 \mathrm{mmol})$ and DMSO $(1.0 \mathrm{~mL})$. The product was purified by flash column chromatography on silica gel (hexane/EtOAc) and obtained a colorless oil ( $0.06 \mathrm{mmol}, 22.3 \mathrm{mg}, 31 \%), \mathrm{R}_{\mathrm{f}}=0.50$ (hexane : EtOAc = 8: 1). ${ }^{1} \mathbf{H}$ NMR $\left(400 \mathrm{MHz}, \mathrm{CDCl}_{3}\right): \delta 8.04(\mathrm{~d}, J=7.2 \mathrm{~Hz}, 2 \mathrm{H}), 7.55(\mathrm{t}, J=7.4 \mathrm{~Hz}, 1 \mathrm{H}), 7.43$ $(\mathrm{t}, J=7.6 \mathrm{~Hz}, 2 \mathrm{H}), 7.40-7.37(\mathrm{~m}, 2 \mathrm{H}), 7.30-7.27(\mathrm{~m}, 3 \mathrm{H}), 4.64(\mathrm{t}, J=6.8 \mathrm{~Hz}, 2 \mathrm{H}), 2.61-2.38(\mathrm{~m}, 2 \mathrm{H})$, 2.26-2.18 (m, 1H), 2.13-2.06 (m, 1H), $1.54(\mathrm{~s}, 3 \mathrm{H}) \mathrm{ppm} .{ }^{13} \mathbf{C}$ NMR (101 MHz, $\left.\mathrm{CDCl}_{3}\right): \delta 166.7,133.2$, $131.8,130.2,129.7,128.5,128.4,128.2,126.1\left(\mathrm{q}, J_{C-F}=279.8 \mathrm{~Hz}\right), 123.0,91.9,83.3,62.2,44.6$ (q, $J_{C-}$ $\left.{ }_{F}=26.9 \mathrm{~Hz}\right), 40.0,31.8,27.2 \mathrm{ppm} .{ }^{19} \mathbf{F}$ NMR $\left(470 \mathrm{MHz}, \mathrm{CDCl}_{3}\right): \delta-61.15(\mathrm{t}, J=11.0 \mathrm{~Hz}, 3 \mathrm{~F}) . \mathbf{H R M S}$ $\mathrm{m} / \mathrm{z}$ (ESI): calcd. for $\mathrm{C}_{21} \mathrm{H}_{19} \mathrm{~F}_{3} \mathrm{O}_{2} \mathrm{Na}[\mathrm{M}+\mathrm{Na}]^{+}: 383.1229$; found: 383.1229 .<smiles>COc1ccc(CC(C#CC(F)(F)c2ccccc2)Cc2ccccc2)cc1</smiles>

3ac: 1-methoxy-4-(4-phenyl-2-(2,2,2-trifluoroethyl)but-3-yn-1-yl)benzene. Prepared according to the general procedure. Reaction was run using $1 \mathrm{ac}(29.6 \mathrm{mg}, 0.2 \mathrm{mmol}), \mathrm{NaOAc}(3.3 \mathrm{mg}, 0.04 \mathrm{mmol})$, $\mathrm{AgOCOCF}_{3}(4.4 \mathrm{mg}, 0.02 \mathrm{mmol}), \mathrm{BIOMe}(22.2 \mathrm{mg}, 0.08 \mathrm{mmol}), \mathrm{Me}_{3} \mathrm{SiCF}_{3}(120 \mu \mathrm{L}, 0.8 \mathrm{mmol}), \mathbf{2 a}$ (208.8 $\mathrm{mg}, 0.6 \mathrm{mmol})$ and DMSO $(1.0 \mathrm{~mL})$. The product was purified by flash column chromatography on silica gel (hexane/EtOAc) and obtained a colorless oil ( $0.14 \mathrm{mmol}, 45.8 \mathrm{mg}, 72 \%), \mathrm{R}_{\mathrm{f}}=0.50$ (hexane : EtOAc $=8: 1) .{ }^{1} \mathbf{H}$ NMR $\left(500 \mathrm{MHz}, \mathrm{CDCl}_{3}\right): \delta 7.41-7.39(\mathrm{~m}, 2 \mathrm{H}), 7.32-7.30(\mathrm{~m}, 3 \mathrm{H}), 7.23(\mathrm{~d}, J=8.5$ $\mathrm{Hz}, 2 \mathrm{H}), 6.90(\mathrm{~d}, J=8.5 \mathrm{~Hz}, 2 \mathrm{H}), 3.82(\mathrm{~s}, 3 \mathrm{H}), 3.21-3.15(\mathrm{~m}, 1 \mathrm{H}), 2.93-2.91(\mathrm{~m}, 2 \mathrm{H}), 2.43-2.31(\mathrm{~m}, 2 \mathrm{H})$ ppm. ${ }^{13} \mathrm{C}$ NMR (101 MHz, $\left.\mathrm{CDCl}_{3}\right): \delta 158.7,131.7,130.6,130.0,128.4,128.1,126.4$ (q, $J_{C-F}=278.6$ $\mathrm{Hz}), 122.3,113.9,89.9,83.5,55.4,40.1,38.2\left(\mathrm{q}, J_{C-F}=27.9 \mathrm{~Hz}\right), 28.6$ ppm. ${ }^{19} \mathbf{F} \mathbf{N M R}\left(470 \mathrm{MHz}, \mathrm{CDCl}_{3}\right)$ : $\delta-64.93(\mathrm{t}, J=10.6 \mathrm{~Hz}, 3 \mathrm{~F})$. HRMS m/z (APCI): calcd. for $\mathrm{C}_{19} \mathrm{H}_{18} \mathrm{~F}_{3} \mathrm{O}[\mathrm{M}+\mathrm{H}]^{+}: 319.1304$; found: 319.1302 .<smiles>FC(F)(F)CC(F)(C#Cc1ccccc1)OC1CCCCC1</smiles>

3ad: (3-(cyclohexyloxy)-5,5,5-trifluoropent-1-yn-1-yl)benzene. Prepared according to the general procedure. Reaction was run using 1ad (25.2 $\mathrm{mg}, 0.2 \mathrm{mmol}$ ), $\mathrm{NaOAc}(3.3 \mathrm{mg}, 0.04 \mathrm{mmol}), \mathrm{AgOCOCF}_{3}$ 
(4.4 mg, $0.02 \mathrm{mmol})$, BIOMe (22.2 mg, $0.08 \mathrm{mmol}), \mathrm{Me}_{3} \mathrm{SiCF}_{3}(120 \mu \mathrm{L}, 0.8 \mathrm{mmol}), 2 \mathrm{a}(208.8 \mathrm{mg}, 0.6$ $\mathrm{mmol}$ ) and DMSO $(1.0 \mathrm{~mL})$. The product was purified by flash column chromatography on silica gel (hexane/EtOAc) and obtained a colorless oil $(0.19 \mathrm{mmol}, 56.2 \mathrm{mg}, 95 \%), \mathrm{R}_{\mathrm{f}}=0.80$ (hexane $:$ EtOAc $=$ 8 : 1). ${ }^{1} \mathbf{H}$ NMR $\left(500 \mathrm{MHz}, \mathrm{CDCl}_{3}\right): \delta$ 7.46-7.44 (m, $\left.2 \mathrm{H}\right), 7.36-7.32(\mathrm{~m}, 3 \mathrm{H}), 4.73-4.70(\mathrm{~m}, 1 \mathrm{H}), 3.73-$ $3.69(\mathrm{~m}, 1 \mathrm{H}), 2.75-2.57(\mathrm{~m}, 2 \mathrm{H}), 1.94(\mathrm{~m}, 1 \mathrm{H}), 1.80-1.75(\mathrm{~m}, 2 \mathrm{H}), 1.55-1.44(\mathrm{~m}, 2 \mathrm{H}), 1.40-1.26(\mathrm{~m}, 5 \mathrm{H})$ ppm. ${ }^{13} \mathrm{C}$ NMR (101 MHz, $\left.\mathrm{CDCl}_{3}\right): \delta 131.9,128.8,128.4,125.5$ (q, $\left.J_{C-F}=278.4 \mathrm{~Hz}\right), 122.4,86.9,85.6$, $76.2,61.3,40.9$ (q, $\left.J_{C-F}=27.9 \mathrm{~Hz}\right), 33.2,31.0,25.8,24.1,23.8 \mathrm{ppm} .{ }^{19} \mathbf{F} \mathbf{N M R}\left(470 \mathrm{MHz}, \mathrm{CDCl}_{3}\right): \delta-$ 64.61 (t, $J=10.3 \mathrm{~Hz}, 3 \mathrm{~F}$ ). HRMS m/z (APCI): calcd. for $\mathrm{C}_{17} \mathrm{H}_{20} \mathrm{~F}_{3} \mathrm{O}[\mathrm{M}+\mathrm{H}]^{+}: 297.1461$; found: 297.1459 .<smiles>CC1CCCCC1C#Cc1ccccc1</smiles>

3ae: (2-(trifluoromethyl)cyclohexyl)ethynyl)benzene. Prepared according to the general procedure. Reaction was run using 1ae (16.4 mg, $0.2 \mathrm{mmol}$ ), NaOAc (3.3 mg, $0.04 \mathrm{mmol}), \mathrm{AgOCOCF}_{3}(4.4 \mathrm{mg}$, $0.02 \mathrm{mmol}$ ), BIOMe (22.2 mg, $0.08 \mathrm{mmol}), \mathrm{Me}_{3} \mathrm{SiCF}_{3}(120 \mu \mathrm{L}, 0.8 \mathrm{mmol}), 2 \mathrm{a}(208.8 \mathrm{mg}, 0.6 \mathrm{mmol})$ and DMSO $(1.0 \mathrm{~mL})$. The product was purified by flash column chromatography on silica gel (hexane/EtOAc) and obtained a colorless oil $(0.11 \mathrm{mmol}, 27.2 \mathrm{mg}, 54 \%, \mathrm{dr}>20: 1), \mathrm{R}_{\mathrm{f}}=0.80$ (hexane : EtOAc $=40: 1)$. Major isomer: ${ }^{1} \mathbf{H}$ NMR $\left(500 \mathrm{MHz}, \mathrm{CDCl}_{3}\right): \delta 7.41-7.39(\mathrm{~m}, 2 \mathrm{H}), 7.30-7.27(\mathrm{~m}, 3 \mathrm{H})$, $2.65(\mathrm{dt}, J=10.5 \mathrm{~Hz}, J=4.0 \mathrm{~Hz}, 1 \mathrm{H}), 2.28-2.20(\mathrm{~m}, 1 \mathrm{H}), 2.20-2.14(\mathrm{~m}, 1 \mathrm{H}), 2.08-2.02(\mathrm{~m}, 1 \mathrm{H}), 1.84-$ $1.76(\mathrm{~m}, 2 \mathrm{H}), 1.58-1.51(\mathrm{~m}, 1 \mathrm{H}), 1.40-1.26(\mathrm{~m}, 3 \mathrm{H}) \mathrm{ppm} .{ }^{13} \mathbf{C} \mathbf{~ N M R}\left(101 \mathrm{MHz}, \mathrm{CDCl}_{3}\right): \delta 131.7,128.3$, 127.9, $127.4\left(\mathrm{q}, J_{C-F}=281.8 \mathrm{~Hz}\right), 123.7,90.8,82.1,46.2\left(\mathrm{q}, J_{C-F}=24.6 \mathrm{~Hz}\right), 32.9,29.5,24.8,24.3 \mathrm{ppm}$. ${ }^{19}$ F NMR (470 MHz, $\mathrm{CDCl}_{3}$ ): $\delta-67.75(\mathrm{~d}, J=8.0 \mathrm{~Hz}, 3 \mathrm{~F})$. HRMS m/z (APCI): calcd. for $\mathrm{C}_{15} \mathrm{H}_{16} \mathrm{~F}_{3}$ $[\mathrm{M}+\mathrm{H}]^{+}:$253.1199; found: 253.1196 .<smiles>CC1CCCOC1=CP</smiles>

3af: (2-(trifluoromethyl)cyclohexyl)ethynyl)benzene. Prepared according to the general procedure. Reaction was run using 1af (16.8 mg, $0.2 \mathrm{mmol}), \mathrm{NaOAc}(3.3 \mathrm{mg}, 0.04 \mathrm{mmol}), \mathrm{AgOCOCF}_{3}(4.4 \mathrm{mg}$, $0.02 \mathrm{mmol}$ ), BIOMe (22.2 mg, $0.08 \mathrm{mmol}), \mathrm{Me}_{3} \mathrm{SiCF}_{3}(120 \mu \mathrm{L}, 0.8 \mathrm{mmol}), \mathbf{2 a}(208.8 \mathrm{mg}, 0.6 \mathrm{mmol})$ and DMSO $(1.0 \mathrm{~mL})$. The product was purified by flash column chromatography on silica gel (hexane/EtOAc) and obtained a colorless oil ( $0.14 \mathrm{mmol}, 36.1 \mathrm{mg}, 71 \%, \mathrm{dr}=9: 1), \mathrm{R}_{\mathrm{f}}=0.60$ (hexane : EtOAc $=40: 1)$. Major isomer: ${ }^{1} \mathbf{H}$ NMR $\left(400 \mathrm{MHz}, \mathrm{CDCl}_{3}\right): \delta 7.47-7.45(\mathrm{~m}, 2 \mathrm{H}), 7.36-7.28(\mathrm{~m}, 3 \mathrm{H})$, $4.57(\mathrm{~d}, J=7.6 \mathrm{~Hz}, 1 \mathrm{H}), 4.08$ (dt, $J=11.6 \mathrm{~Hz}, J=4.4 \mathrm{~Hz}, 1 \mathrm{H}), 3.57$ (dt, $J=10.4 \mathrm{~Hz}, J=2.8 \mathrm{~Hz}, 1 \mathrm{H})$, 2.55-2.43 (m, 1H), 2.22-2.14 (m, 1H), 1.84-1.63 (m, 3H) ppm. ${ }^{13} \mathbf{C}$ NMR (126 MHz, $\left.\mathrm{CDCl}_{3}\right): \delta 131.9$, 128.8, 128.4, $127.6\left(\mathrm{q}, J_{C-F}=280.9 \mathrm{~Hz}\right), 122.3,87.0,85.2,66.5,66.3,44.7\left(\mathrm{q}, J_{C-F}=25.1 \mathrm{~Hz}\right), 23.3,21.7$ ppm. ${ }^{19} \mathbf{F}$ NMR (470 MHz, $\left.\mathrm{CDCl}_{3}\right): \delta-67.75$ (d, $\left.J=8.9 \mathrm{~Hz}, 3 \mathrm{~F}\right)$. HRMS m/z (APCI): calcd. for $\mathrm{C}_{14} \mathrm{H}_{14} \mathrm{~F}_{3} \mathrm{O}[\mathrm{M}+\mathrm{H}]^{+}: 255.0991$; found: 255.0989 .<smiles>CCC(C#CCC(C)(F)F)C(F)(F)F</smiles>

3ag: (4-methyl-3-(2,2,2-trifluoroethyl)hex-1-yn-1-yl)benzene. Prepared according to the general 
procedure. Reaction was run using $1 \mathrm{ag}$ (16.8 $\mathrm{mg}, 0.2 \mathrm{mmol}$ ), $\mathrm{NaOAc}(3.3 \mathrm{mg}, 0.04 \mathrm{mmol}), \mathrm{AgOCOCF}_{3}$ (4.4 mg, $0.02 \mathrm{mmol}$ ), BIOMe (22.2 mg, $0.08 \mathrm{mmol}), \mathrm{Me}_{3} \mathrm{SiCF}_{3}(120 \mu \mathrm{L}, 0.8 \mathrm{mmol}), 2 \mathrm{a}(208.8 \mathrm{mg}, 0.6$ $\mathrm{mmol}$ ) and DMSO $(1.0 \mathrm{~mL})$. The product was purified by flash column chromatography on silica gel (hexane/EtOAc) and obtained a colorless oil $(0.06 \mathrm{mmol}, 15.3 \mathrm{mg}, 30 \%, \mathrm{dr}=1.4: 1), \mathrm{R}_{\mathrm{f}}=0.40$ (hexane).

Major isomer: ${ }^{1} \mathbf{H}$ NMR $\left(400 \mathrm{MHz}, \mathrm{CDCl}_{3}\right): \delta$ 7.42-7.40 (m, $\left.2 \mathrm{H}\right), 7.31-7.28(\mathrm{~m}, 3 \mathrm{H}), 2.93-2.89(\mathrm{~m}, 1 \mathrm{H})$, 2.50-2.22 (m, 2H), 1.61-1.54 (m, 1H), 1.45-1.34 (m, 2H), 1.07 (d, $J=6.8 \mathrm{~Hz}, 3 \mathrm{H}), 0.96(\mathrm{t}, J=7.4 \mathrm{~Hz}$ $3 \mathrm{H})$ ppm. ${ }^{13} \mathbf{C}$ NMR $\left(101 \mathrm{MHz}, \mathrm{CDCl}_{3}\right): \delta 131.7,128.3,128.0,126.8$ (q, $\left.J_{C-F}=278.4 \mathrm{~Hz}\right), 123.7,89.4$, 83.3, 38.7, 36.4 (q, $\left.J_{C-F}=27.7 \mathrm{~Hz}\right), 32.0,25.4,17.1,11.9$ ppm. ${ }^{19} \mathbf{F}$ NMR $\left(470 \mathrm{MHz}, \mathrm{CDCl}_{3}\right): \delta-65.37$ (t, $J=10.6 \mathrm{~Hz}, 3 \mathrm{~F})$. Minor isomer: ${ }^{1} \mathbf{H}$ NMR $\left(400 \mathrm{MHz}, \mathrm{CDCl}_{3}\right): \delta 7.42-7.40(\mathrm{~m}, 2 \mathrm{H}), 7.31-7.28(\mathrm{~m}$, $3 \mathrm{H}), 3.05-3.00(\mathrm{~m}, 1 \mathrm{H}), 2.50-2.22(\mathrm{~m}, 2 \mathrm{H}), 1.70-1.64(\mathrm{~m}, 1 \mathrm{H}), 1.56-1.50(\mathrm{~m}, 2 \mathrm{H}), 1.02(\mathrm{~d}, J=6.4 \mathrm{~Hz}$, $3 \mathrm{H}), 0.97$ (t, $J=7.2 \mathrm{~Hz} 3 \mathrm{H})$ ppm. ${ }^{13} \mathbf{C}$ NMR $\left(101 \mathrm{MHz}, \mathrm{CDCl}_{3}\right): \delta 131.8,128.3,128.0,126.7$ (q, $J_{C-F}=$ $278.5 \mathrm{~Hz}), 123.6,88.3,83.6,38.5,37.7$ (q, $\left.J_{C-F}=27.6 \mathrm{~Hz}\right), 31.0,28.2,14.9,11.9$ ppm. ${ }^{19} \mathbf{F}$ NMR $(470$ $\left.\mathrm{MHz}, \mathrm{CDCl}_{3}\right): \delta-65.40(\mathrm{t}, J=10.8 \mathrm{~Hz}, 3 \mathrm{~F})$. HRMS m/z (APCI): calcd. for $\mathrm{C}_{15} \mathrm{H}_{18} \mathrm{~F}_{3}[\mathrm{M}+\mathrm{H}]^{+}: 255.1355$; found: 255.1355 .<smiles>O=C1CCCN1C(=C=Cc1ccccc1)C(F)(F)F</smiles>

3ah: 1-(5,5,5-trifluoro-1-phenylpent-1-yn-3-yl)pyrrolidin-2-one. Prepared according to the general procedure. Reaction was run using 1ah (22.2 mg, $0.2 \mathrm{mmol}$ ), $\mathrm{NaOAc}$ (3.3 mg, $0.04 \mathrm{mmol}), \mathrm{AgOCOCF}_{3}$ (4.4 mg, $0.02 \mathrm{mmol}$ ), BIOMe (22.2 mg, $0.08 \mathrm{mmol}), \mathrm{Me}_{3} \mathrm{SiCF}_{3}(120 \mu \mathrm{L}, 0.8 \mathrm{mmol}), 2 \mathrm{a}(208.8 \mathrm{mg}, 0.6$ $\mathrm{mmol})$ and DMSO $(1.0 \mathrm{~mL})$. The product was purified by flash column chromatography on silica gel (hexane/EtOAc) and obtained a colorless oil $(0.19 \mathrm{mmol}, 53.4 \mathrm{mg}, 30 \%), \mathrm{R}_{\mathrm{f}}=0.30$ (hexane $:$ EtOAc $=$ 2 : 1). ${ }^{1} \mathbf{H}$ NMR $\left(500 \mathrm{MHz}, \mathrm{CDCl}_{3}\right): \delta 7.41-7.39(\mathrm{~m}, 2 \mathrm{H}), 7.32-7.27(\mathrm{~m}, 3 \mathrm{H}), 5.55(\mathrm{t}, J=7.0 \mathrm{~Hz}, 1 \mathrm{H})$, 3.62 (q, $J=8.0 \mathrm{~Hz}, 1 \mathrm{H}), 3.42$ (q, $J=8.0 \mathrm{~Hz}, 1 \mathrm{H}), 2.63-2.57(\mathrm{~m}, 2 \mathrm{H}), 2.41$ (t, $J=8.0 \mathrm{~Hz}, 2 \mathrm{H}), 2.11-2.00$ (m, 2H). ${ }^{13}$ C NMR (126 MHz, $\left.\mathrm{CDCl}_{3}\right): \delta 174.4,131.2,128.9,128.4,125.0$ (q, $\left.J_{C-F}=278.0 \mathrm{~Hz}\right), 121.8$, 85.4, 83.5, 43.2, 38.8, 37.1 (q, $\left.J_{C-F}=28.2 \mathrm{~Hz}\right), 30.9,17.9$ ppm. ${ }^{19} \mathbf{F}$ NMR $\left(470 \mathrm{MHz}, \mathrm{CDCl}_{3}\right): \delta-65.61$ (t, $J=10.1 \mathrm{~Hz}, 3 \mathrm{~F}$ ). HRMS m/z (APCI): calcd. for $\mathrm{C}_{15} \mathrm{H}_{15} \mathrm{~F}_{3} \mathrm{NO}[\mathrm{M}+\mathrm{H}]^{+}: 282.1100$; found: 282.1099 .<smiles></smiles>

3ai: $\quad$ (8R,9S,13S,14S)-13-methyl-3-((7-phenyl-5-(2,2,2-trifluoroethyl)hept-6-yn-1-yl)oxy)$\mathbf{6 , 7 , 8 , 9 , 1 1 , 1 2 , 1 3 , 1 4 , 1 5 , 1 6 - d e c a h y d r o - 1 7 H - c y c l o p e n t a [ a ] p h e n a n t h r e n - 1 7 - o n e . ~ P r e p a r e d ~ a c c o r d i n g ~ t o ~}$ the general procedure. Reaction was run using 1 ai $(70.4 \mathrm{mg}, 0.2 \mathrm{mmol}), \mathrm{NaOAc}(3.3 \mathrm{mg}, 0.04 \mathrm{mmol})$, $\mathrm{AgOCOCF}_{3}(4.4 \mathrm{mg}, 0.02 \mathrm{mmol}), \mathrm{BIOMe}(22.2 \mathrm{mg}, 0.08 \mathrm{mmol}), \mathrm{Me}_{3} \mathrm{SiCF}_{3}(120 \mu \mathrm{L}, 0.8 \mathrm{mmol}), 2 \mathrm{a}$ $(208.8 \mathrm{mg}, 0.6 \mathrm{mmol})$ and DMSO $(1.0 \mathrm{~mL})$. The product was purified by flash column chromatography on silica gel (hexane/EtOAc) and obtained a colorless oil $(0.11 \mathrm{mmol}, 55.3 \mathrm{mg}, 53 \%), \mathrm{R}_{\mathrm{f}}=0.40$ (hexane : EtOAc $=5: 1) .{ }^{1} \mathbf{H}$ NMR $\left(500 \mathrm{MHz}, \mathrm{CDCl}_{3}\right): \delta 4.43-4.41(\mathrm{~m}, 2 \mathrm{H}), 4.32-4.29(\mathrm{~m}, 3 \mathrm{H}), 7.21(\mathrm{~d}, J=9.0$ $\mathrm{Hz}, 1 \mathrm{H}), 6.73(\mathrm{~d}, J=9.0 \mathrm{~Hz}, 1 \mathrm{H}), 6.67(\mathrm{~s}, 1 \mathrm{H}), 3.99(\mathrm{t}, J=6.0 \mathrm{~Hz}, 2 \mathrm{H}), 3.01-2.95(\mathrm{~m}, 1 \mathrm{H}), 2.93-2.87(\mathrm{~m}$, $2 \mathrm{H}), 2.55-2.40(\mathrm{~m}, 3 \mathrm{H}), 2.37-2.23(\mathrm{~m}, 2 \mathrm{H}), 2.20-1.93(\mathrm{~m}, 4 \mathrm{H}), 1.92-1.78(\mathrm{~m}, 3 \mathrm{H}), 1.76-1.41(\mathrm{~m}, 9 \mathrm{H})$, 0.92 (s, 3H) ppm. ${ }^{13} \mathbf{C}$ NMR $\left(101 \mathrm{MHz}, \mathrm{CDCl}_{3}\right): \delta 221.1,157.1,137.8,132.1,131.7,128.3,128.1,126.4$, 
$126.3\left(\mathrm{q}, J_{C-F}=278.6 \mathrm{~Hz}\right), 123.4,114.6,112.2,89.9,82.9,67.6,50.5,48.1,44.1,39.2\left(\mathrm{q}, J_{C-F}=27.8 \mathrm{~Hz}\right)$, $38.4,36.0,34.6,31.7,31.2,29.7,29.0,26.6,26.5,26.0,23.8,21.7,13.9$ ppm. ${ }^{19} \mathbf{F}$ NMR (470 MHz, $\left.\mathrm{CDCl}_{3}\right): \delta-65.13(\mathrm{t}, J=10.6,3 \mathrm{~F}) \mathrm{ppm}$. HRMS m/z (ESI): calcd. for $\mathrm{C}_{33} \mathrm{H}_{37} \mathrm{~F}_{3} \mathrm{O}_{2} \mathrm{Na}[\mathrm{M}+\mathrm{Na}]^{+}: 545.2638$; found: 545.2638 .<smiles>Cc1cc(=O)oc2cc(OCCCCC(C#Cc3ccccc3)CC(F)(F)F)ccc12</smiles>

3aj: $\quad$ 4-methyl-7-((7-phenyl-5-(2,2,2-trifluoroethyl)hept-6-yn-1-yl)oxy)-2H-chromen-2-one. Prepared according to the general procedure. Reaction was run using 1aj (51.6 mg, $0.2 \mathrm{mmol}$ ), NaOAc (3.3 mg, $0.04 \mathrm{mmol}), \mathrm{AgOCOCF}_{3}(4.4 \mathrm{mg}, 0.02 \mathrm{mmol}), \mathrm{BIOMe}(22.2 \mathrm{mg}, 0.08 \mathrm{mmol}), \mathrm{Me}_{3} \mathrm{SiCF}_{3}(120$ $\mu \mathrm{L}, 0.8 \mathrm{mmol}), 2 \mathrm{a}(208.8 \mathrm{mg}, 0.6 \mathrm{mmol})$ and DMSO $(1.0 \mathrm{~mL})$. The product was purified by flash column chromatography on silica gel (hexane/EtOAc) and obtained a colorless oil (0.11 mmol, $47.9 \mathrm{mg}, 56 \%)$, $\mathrm{R}_{\mathrm{f}}=0.20$ (hexane : EtOAc $\left.=5: 1\right) .{ }^{1} \mathbf{H}$ NMR $\left(400 \mathrm{MHz}, \mathrm{CDCl}_{3}\right): \delta 7.46(\mathrm{~d}, J=8.8 \mathrm{~Hz}, 1 \mathrm{H}), 7.40-7.37$ $(\mathrm{m}, 2 \mathrm{H}), 7.30-7.26(\mathrm{~m}, 3 \mathrm{H}), 6.84(\mathrm{dd}, J=8.8 \mathrm{~Hz}, J=2.4 \mathrm{~Hz}, 1 \mathrm{H}), 6.79(\mathrm{~d}, J=2.4 \mathrm{~Hz}, 1 \mathrm{H}), 6.12(\mathrm{~s}, 1 \mathrm{H})$, $4.04(\mathrm{t}, J=6.0 \mathrm{~Hz}, 2 \mathrm{H}), 3.00-2.94(\mathrm{~m}, 1 \mathrm{H}), 2.54-2.40(\mathrm{~m}, 1 \mathrm{H}), 2.38(\mathrm{~s}, 3 \mathrm{H}), 2.38-2.26(\mathrm{~m}, 1 \mathrm{H}), 1.94-$ $1.78(\mathrm{~m}, 3 \mathrm{H}), 1.77-1.65$ (m, 3H) ppm. ${ }^{13} \mathbf{C}$ NMR (101 MHz, $\left.\mathrm{CDCl}_{3}\right): \delta 162.1,161.5,155.3,152.7,131.7$, $128.3,128.1,126.3\left(\mathrm{q}, J_{C-F}=278.6 \mathrm{~Hz}\right), 125.6,123.2,113.6,112.7,111.9,101.4,89.7,83.0,68.3,39.2$ $\left(\mathrm{q}, J_{C-F}=27.8 \mathrm{~Hz}\right), 34.5,28.7,26.5,23.7,18.8 \mathrm{ppm} .{ }^{19} \mathbf{F} \mathbf{N M R}\left(470 \mathrm{MHz}, \mathrm{CDCl}_{3}\right): \delta-65.11(\mathrm{t}, J=10.6$, $3 \mathrm{~F})$ ppm. HRMS m/z (ESI): calcd. for $\mathrm{C}_{25} \mathrm{H}_{23} \mathrm{~F}_{3} \mathrm{O}_{3} \mathrm{Na}[\mathrm{M}+\mathrm{Na}]^{+}: 451.1492$; found: 451.1489 .<smiles>O=c1c(OCCCCC(C#Cc2ccccc2)CC(F)(F)F)c(-c2ccccc2)oc2ccccc12</smiles>

3ak: 2-phenyl-3-((7-phenyl-5-(2,2,2-trifluoroethyl)hept-6-yn-1-yl)oxy)-4H-chromen-4-one. Prepared according to the general procedure. Reaction was run using 1ak (51.6 mg, $0.2 \mathrm{mmol}$ ), NaOAc (3.3 mg, $0.04 \mathrm{mmol}), \mathrm{AgOCOCF}_{3}$ (4.4 mg, $0.02 \mathrm{mmol}$ ), BIOMe (22.2 mg, $\left.0.08 \mathrm{mmol}\right), \mathrm{Me}_{3} \mathrm{SiCF}_{3}(120$ $\mu \mathrm{L}, 0.8 \mathrm{mmol}), 2 \mathrm{a}(208.8 \mathrm{mg}, 0.6 \mathrm{mmol})$ and DMSO $(1.0 \mathrm{~mL})$. The product was purified by flash column chromatography on silica gel (hexane/EtOAc) and obtained a colorless oil (0.13 mmol, $63.7 \mathrm{mg}, 65 \%)$, $\mathrm{R}_{\mathrm{f}}=0.40$ (hexane : EtOAc $\left.=5: 1\right) .{ }^{1} \mathbf{H} \mathbf{N M R}\left(500 \mathrm{MHz}, \mathrm{CDCl}_{3}\right): \delta 8.29(\mathrm{dd}, J=8.0 \mathrm{~Hz}, J=1.5 \mathrm{~Hz}, 1 \mathrm{H})$, $8.12(\mathrm{dd}, J=8.0 \mathrm{~Hz}, J=1.5 \mathrm{~Hz}, 2 \mathrm{H}), 7.69$ (t, $J=7.8 \mathrm{~Hz}, 1 \mathrm{H}), 7.56-7.49$ (m, 4H), 7.43-7.40 (m, 3H), 7.31-7.28 (m, 3H), $4.12(\mathrm{t}, J=6.5 \mathrm{~Hz}, 2 \mathrm{H}), 2.92-2.87(\mathrm{~m}, 1 \mathrm{H}), 2.47-2.35(\mathrm{~m}, 1 \mathrm{H}), 2.31-2.21(\mathrm{~m}, 1 \mathrm{H})$, 1.84-1.77 (m, 2H), 1.74-1.68 (m, 1H), 1.65-1.58 (m, 2H) ppm. ${ }^{13} \mathbf{C}$ NMR (126 MHz, CDCl $): \delta 175.3$, 156.0, 155.4, 140.7, 133.5, 131.7, 131.1, 130.8, 128.8, 128.5, 128.3, 128.0, 126.3 (q, $J_{C-F}=278.0 \mathrm{~Hz}$ ), $125.9,124.8,124.3,123.4,118.1,90.0,82.8,72.5,39.1$ (q, $J_{C-F}=27.6 \mathrm{~Hz}$ ), 34.6, 29.8, 26.5, $23.6 \mathrm{ppm}$. ${ }^{19}$ F NMR $\left(470 \mathrm{MHz}, \mathrm{CDCl}_{3}\right): \delta-65.13(\mathrm{t}, J=10.6,3 \mathrm{~F}) \mathrm{ppm}$. HRMS m/z (ESI): calcd. for $\mathrm{C}_{30} \mathrm{H}_{25} \mathrm{~F}_{3} \mathrm{O}_{2} \mathrm{Na}$ $[\mathrm{M}+\mathrm{Na}]^{+}:$513.1648; found: 513.1650 .

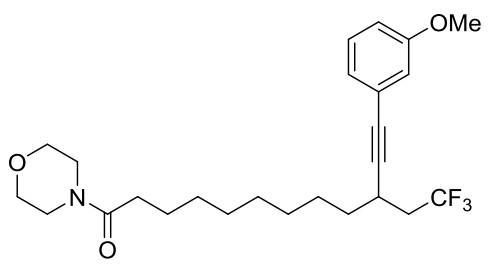


3al: 12-(3-methoxyphenyl)-1-morpholino-10-(2,2,2-trifluoroethyl)dodec-11-yn-1-one. Prepared according to the general procedure. Reaction was run using $1 \mathbf{y}(50.6 \mathrm{mg}, 0.2 \mathrm{mmol}), \mathrm{NaOAc}(3.3 \mathrm{mg}$, $0.04 \mathrm{mmol}), \mathrm{AgOCOCF}_{3}(4.4 \mathrm{mg}, 0.02 \mathrm{mmol}), \mathrm{BIOMe}(22.2 \mathrm{mg}, 0.08 \mathrm{mmol}), \mathrm{Me}_{3} \mathrm{SiCF}_{3}(120 \mu \mathrm{L}, 0.8$ $\mathrm{mmol}), 2 \mathbf{b}(226.8 \mathrm{mg}, 0.6 \mathrm{mmol})$ and DMSO $(1.0 \mathrm{~mL})$. The product was purified by flash column chromatography on silica gel (hexane/EtOAc) and obtained a colorless oil (0.12 mmol, $56.2 \mathrm{mg}, 62 \%)$, $\mathrm{R}_{\mathrm{f}}=0.2$ (hexane : EtOAc = 2 : 1). ${ }^{1} \mathbf{H}$ NMR $\left(400 \mathrm{MHz}, \mathrm{CDCl}_{3}\right): \delta 7.17(\mathrm{t}, J=7.6 \mathrm{~Hz}, 1 \mathrm{H}), 6.97(\mathrm{~d}, J=$ $7.6 \mathrm{~Hz}, 1 \mathrm{H}), 6.90(\mathrm{~s}, 1 \mathrm{H}), 6.82(\mathrm{~d}, J=7.6 \mathrm{~Hz}, 1 \mathrm{H}), 3.77(\mathrm{~s}, 3 \mathrm{H}), 3.62(\mathrm{~m}, 4 \mathrm{H}), 3.59(\mathrm{~m}, 2 \mathrm{H}), 3.42(\mathrm{~m}$, $2 \mathrm{H}), 2.92-2.85(\mathrm{~m}, 1 \mathrm{H}), 2.47-2.33(\mathrm{~m}, 1 \mathrm{H}), 2.33-2.21(\mathrm{~m}, 1 \mathrm{H}), 2.27(\mathrm{t}, J=7.2 \mathrm{~Hz}, 2 \mathrm{H}), 1.63-1.52(\mathrm{~m}$, 5H), 1.48-1.40 (m, 1H), 1.36-1.25 (m, 8H) ppm. ${ }^{13} \mathbf{C}$ NMR (101 MHz, $\left.\mathrm{CDCl}_{3}\right): \delta 171.9,159.3,129.3$, $126.3\left(\mathrm{q}, J_{C-F}=278.6 \mathrm{~Hz}\right), 124.4,124.2,116.5,114.4,90.1,82.5,67.0,66.7,55.3,46.1,41.9,39.1\left(\mathrm{q}, J_{C-}\right.$ $\left.{ }_{F}=27.6 \mathrm{~Hz}\right), 34.8,33.1,29.5,29.4,29.4,29.2,26.9,26.5$ (q, $\left.J_{C-F}=2.9 \mathrm{~Hz}\right), 25.3$ ppm. ${ }^{19} \mathbf{F}$ NMR $(470$ $\left.\mathrm{MHz}, \mathrm{CDCl}_{3}\right): \delta-65.15(\mathrm{t}, J=10.6 \mathrm{~Hz}, 3 \mathrm{~F})$. HRMS m/z (ESI): calcd. for $\mathrm{C}_{25} \mathrm{H}_{34} \mathrm{~F}_{3} \mathrm{NO}_{3} \mathrm{Na}[\mathrm{M}+\mathrm{Na}]^{+}$: 476.2383; found: 476.2383 .

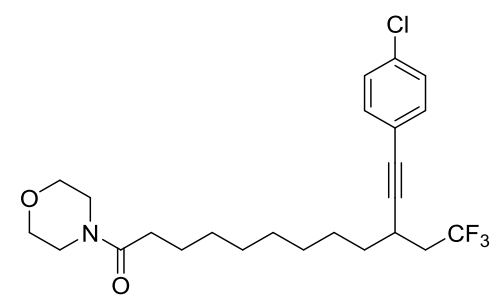

3am: 12-(4-chlorophenyl)-1-morpholino-10-(2,2,2-trifluoroethyl)dodec-11-yn-1-one. Prepared according to the general procedure. Reaction was run using $1 \mathbf{y}(50.6 \mathrm{mg}, 0.2 \mathrm{mmol}), \mathrm{NaOAc}(3.3 \mathrm{mg}$, $0.04 \mathrm{mmol}$ ), $\mathrm{AgOCOCF}_{3}$ (4.4 mg, $0.02 \mathrm{mmol}$ ), BIOMe (22.2 mg, $\left.0.08 \mathrm{mmol}\right), \mathrm{Me}_{3} \mathrm{SiCF}_{3}(120 \mu \mathrm{L}, 0.8$ $\mathrm{mmol}), 2 \mathrm{c}(229.2 \mathrm{mg}, 0.6 \mathrm{mmol})$ and DMSO $(1.0 \mathrm{~mL})$. The product was purified by flash column chromatography on silica gel (hexane/EtOAc) and obtained a colorless oil (0.17 mmol, $77.7 \mathrm{mg}, 85 \%)$, $\mathrm{R}_{\mathrm{f}}=0.3$ (hexane : Acetone $\left.=3: 1\right) .{ }^{1} \mathbf{H}$ NMR $\left(500 \mathrm{MHz}, \mathrm{CDCl}_{3}\right): \delta 7.32(\mathrm{~d}, J=8.0 \mathrm{~Hz}, 2 \mathrm{H}), 7.26(\mathrm{~d}, J=$ $8.5 \mathrm{~Hz}, 2 \mathrm{H}), 3.66(\mathrm{~m}, 4 \mathrm{H}), 3.62(\mathrm{~m}, 2 \mathrm{H}), 3.46(\mathrm{~m}, 2 \mathrm{H}), 2.93-2.88(\mathrm{~m}, 1 \mathrm{H}), 2.47-2.36(\mathrm{~m}, 1 \mathrm{H}), 2.34-2.25$ $(\mathrm{m}, 1 \mathrm{H}), 2.30(\mathrm{t}, J=7.8 \mathrm{~Hz}, 2 \mathrm{H}), 1.64-1.55(\mathrm{~m}, 5 \mathrm{H}), 1.50-1.43(\mathrm{~m}, 1 \mathrm{H}), 1.39-1.29(\mathrm{~m}, 8 \mathrm{H}) \mathrm{ppm} .{ }^{13} \mathrm{C}$ NMR (126 MHz, $\left.\mathrm{CDCl}_{3}\right): \delta 171.9,133.9,132.9,128.6,126.3\left(\mathrm{q}, J_{C-F}=280.0 \mathrm{~Hz}\right), 121.9,91.3,81.5$, 67.0, 66.7, 46.1, 41.9, 39.1 (q, $\left.J_{C-F}=27.7 \mathrm{~Hz}\right), 34.8,33.1,29.5,29.4,29.4,29.2,27.0,26.4\left(\mathrm{q}, J_{C-F}=2.9\right.$ $\mathrm{Hz}), 25.2$ ppm. ${ }^{19} \mathbf{F}$ NMR (470 MHz, $\left.\mathrm{CDCl}_{3}\right): \delta-65.18$ (t, $\left.J=10.6 \mathrm{~Hz}, 3 \mathrm{~F}\right)$. HRMS m/z (ESI): calcd. for $\mathrm{C}_{24} \mathrm{H}_{31} \mathrm{ClF}_{3} \mathrm{NO}_{2} \mathrm{Na}[\mathrm{M}+\mathrm{Na}]^{+}: 480.1888$; found: 480.1886 .

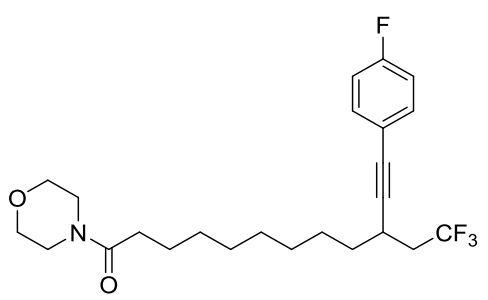

3an: 12-(4-fluorophenyl)-1-morpholino-10-(2,2,2-trifluoroethyl)dodec-11-yn-1-one. Prepared according to the general procedure. Reaction was run using $1 \mathbf{y}(50.6 \mathrm{mg}, 0.2 \mathrm{mmol}), \mathrm{NaOAc}(3.3 \mathrm{mg}$, $0.04 \mathrm{mmol}$ ), $\mathrm{AgOCOCF}_{3}(4.4 \mathrm{mg}, 0.02 \mathrm{mmol}), \mathrm{BIOMe}(22.2 \mathrm{mg}, 0.08 \mathrm{mmol}), \mathrm{Me}_{3} \mathrm{SiCF}_{3}(120 \mu \mathrm{L}, 0.8$ $\mathrm{mmol}), 2 \mathrm{~d}(219.6 \mathrm{mg}, 0.6 \mathrm{mmol})$ and DMSO $(1.0 \mathrm{~mL})$. The product was purified by flash column chromatography on silica gel (hexane/EtOAc) and obtained a colorless oil ( $0.18 \mathrm{mmol}, 77.6 \mathrm{mg}, 88 \%)$, 
$\mathrm{R}_{\mathrm{f}}=0.3$ (hexane : Acetone $\left.=2.5: 1\right) .{ }^{1} \mathbf{H}$ NMR $\left(400 \mathrm{MHz}, \mathrm{CDCl}_{3}\right): \delta 7.36-7.32(\mathrm{~m}, 2 \mathrm{H}), 6.95(\mathrm{t}, J=8.8$ $\mathrm{Hz}, 2 \mathrm{H}), 3.63(\mathrm{~m}, 4 \mathrm{H}), 3.59(\mathrm{~m}, 2 \mathrm{H}), 3.42(\mathrm{~m}, 2 \mathrm{H}), 2.91-2.83(\mathrm{~m}, 1 \mathrm{H}), 2.44-2.32(\mathrm{~m}, 1 \mathrm{H}), 2.31-2.19(\mathrm{~m}$, $1 \mathrm{H}), 2.27(\mathrm{t}, J=7.8 \mathrm{~Hz}, 2 \mathrm{H}), 1.62-1.50(\mathrm{~m}, 5 \mathrm{H}), 1.47-1.40(\mathrm{~m}, 1 \mathrm{H}), 1.34-1.25(\mathrm{~m}, 8 \mathrm{H}) \mathrm{ppm} .{ }^{13} \mathbf{C}$ NMR $\left(126 \mathrm{MHz}, \mathrm{CDCl}_{3}\right): \delta 171.9,162.3\left(\mathrm{~d}, J_{C-F}=249.1 \mathrm{~Hz}\right), 133.5\left(\mathrm{~d}, J_{C-F}=8.3 \mathrm{~Hz}\right), 126.3\left(\mathrm{q}, J_{C-F}=278.0\right.$ $\mathrm{Hz}), 119.5\left(\mathrm{~d}, J_{C-F}=3.5 \mathrm{~Hz}\right), 115.5\left(\mathrm{~d}, J_{C-F}=22.1 \mathrm{~Hz}\right), 89.9,81.5,67.0,66.7,46.1,41.9,39.1\left(\mathrm{q}, J_{C-F}=\right.$ $27.6 \mathrm{~Hz}), 34.8,33.1,29.5,29.4,29.4,29.2,27.0,26.4$ (q, $\left.J_{C-F}=2.8 \mathrm{~Hz}\right), 25.2$ ppm. ${ }^{19} \mathbf{F}$ NMR (470 MHz, $\left.\mathrm{CDCl}_{3}\right): \delta-65.18(\mathrm{t}, J=10.6 \mathrm{~Hz}, 3 \mathrm{~F}),-112.7$ (m, $\left.1 \mathrm{~F}\right)$. HRMS m/z (ESI): calcd. for $\mathrm{C}_{24} \mathrm{H}_{31} \mathrm{~F}_{4} \mathrm{NO}_{2} \mathrm{Na}$ $[\mathrm{M}+\mathrm{Na}]^{+}:$: 464.2183; found: 464.2182 .

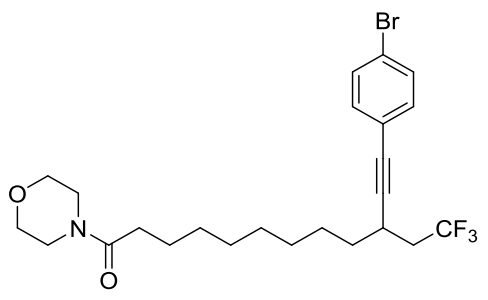

3ao: 12-(4-bromophenyl)-1-morpholino-10-(2,2,2-trifluoroethyl)dodec-11-yn-1-one. Prepared according to the general procedure. Reaction was run using $1 \mathbf{y}(50.6 \mathrm{mg}, 0.2 \mathrm{mmol}), \mathrm{NaOAc}(3.3 \mathrm{mg}$, $0.04 \mathrm{mmol}$ ), $\mathrm{AgOCOCF}_{3}(4.4 \mathrm{mg}, 0.02 \mathrm{mmol}$ ), BIOMe (22.2 mg, $0.08 \mathrm{mmol}), \mathrm{Me}_{3} \mathrm{SiCF}_{3}(120 \mu \mathrm{L}, 0.8$ $\mathrm{mmol}), 2 \mathrm{e}(255.6 \mathrm{mg}, 0.6 \mathrm{mmol})$ and DMSO $(1.0 \mathrm{~mL})$. The product was purified by flash column chromatography on silica gel (hexane/EtOAc) and obtained a colorless oil $(0.16 \mathrm{mmol}, 81.2 \mathrm{mg}, 81 \%)$, $\mathrm{R}_{\mathrm{f}}=0.3$ (hexane : Acetone = 3: 1). ${ }^{1} \mathbf{H}$ NMR $\left(500 \mathrm{MHz}, \mathrm{CDCl}_{3}\right): \delta 7.38(\mathrm{~d}, J=8.0 \mathrm{~Hz}, 2 \mathrm{H}), 7.21(\mathrm{~d}, J=$ $8.0 \mathrm{~Hz}, 2 \mathrm{H}), 3.61(\mathrm{~m}, 4 \mathrm{H}), 3.57(\mathrm{~m}, 2 \mathrm{H}), 3.41(\mathrm{~m}, 2 \mathrm{H}), 2.88-2.84(\mathrm{~m}, 1 \mathrm{H}), 2.43-2.32(\mathrm{~m}, 1 \mathrm{H}), 2.31-2.20$ $(\mathrm{m}, 1 \mathrm{H}), 2.26(\mathrm{t}, J=7.8 \mathrm{~Hz}, 2 \mathrm{H}), 1.61-1.51(\mathrm{~m}, 5 \mathrm{H}), 1.46-1.38(\mathrm{~m}, 1 \mathrm{H}), 1.35-1.24(\mathrm{~m}, 8 \mathrm{H}) \mathrm{ppm} .{ }^{13} \mathrm{C}$ NMR $\left(126 \mathrm{MHz}, \mathrm{CDCl}_{3}\right): \delta 171.9,133.1,131.5,126.2\left(\mathrm{q}, J_{C-F}=280.0 \mathrm{~Hz}\right), 122.4,122.1,91.4,81.6$, 67.0, 66.7, 46.0, 41.9, 39.0 (q, $\left.J_{C-F}=27.7 \mathrm{~Hz}\right), 34.7,33.1,29.4,29.4,29.3,29.2,26.9,26.5$ (q, $J_{C-F}=2.8$ $\mathrm{Hz}), 25.2$ ppm. ${ }^{19} \mathbf{F}$ NMR $\left(470 \mathrm{MHz}, \mathrm{CDCl}_{3}\right): \delta-65.19$ (t, $\left.J=10.8 \mathrm{~Hz}, 3 \mathrm{~F}\right)$. HRMS m/z (ESI): calcd. for $\mathrm{C}_{24} \mathrm{H}_{31} \mathrm{BrF}_{3} \mathrm{NO}_{2} \mathrm{Na}[\mathrm{M}+\mathrm{Na}]^{+}:$:524.1383; found: 524.1383 .

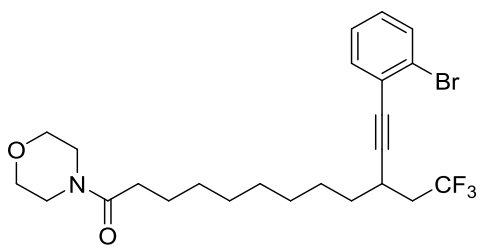

3ap: 12-(2-bromophenyl)-1-morpholino-10-(2,2,2-trifluoroethyl)dodec-11-yn-1-one. Prepared according to the general procedure. Reaction was run using $1 \mathbf{y}(50.6 \mathrm{mg}, 0.2 \mathrm{mmol}), \mathrm{NaOAc}(3.3 \mathrm{mg}$, $0.04 \mathrm{mmol}$ ), $\mathrm{AgOCOCF}_{3}(4.4 \mathrm{mg}, 0.02 \mathrm{mmol}$ ), BIOMe (22.2 mg, $0.08 \mathrm{mmol}), \mathrm{Me}_{3} \mathrm{SiCF}_{3}(120 \mu \mathrm{L}, 0.8$ $\mathrm{mmol}), 2 \mathrm{f}(255.6 \mathrm{mg}, 0.6 \mathrm{mmol})$ and DMSO $(1.0 \mathrm{~mL})$. The product was purified by flash column chromatography on silica gel (hexane/EtOAc) and obtained a colorless oil $(0.14 \mathrm{mmol}, 72.1 \mathrm{mg}, 72 \%)$, $\mathrm{R}_{\mathrm{f}}=0.3$ (hexane : EtOAc $\left.=3: 1\right) .{ }^{1} \mathbf{H}$ NMR $\left(500 \mathrm{MHz}, \mathrm{CDCl}_{3}\right): \delta 7.53(\mathrm{~d}, J=8.0 \mathrm{~Hz}, 1 \mathrm{H}), 7.40(\mathrm{~d}, J=$ $7.5 \mathrm{~Hz}, 1 \mathrm{H}), 7.21(\mathrm{t}, J=7.5 \mathrm{~Hz}, 1 \mathrm{H}), 7.11(\mathrm{t}, J=7.8 \mathrm{~Hz}, 1 \mathrm{H}), 3.62(\mathrm{~m}, 4 \mathrm{H}), 3.59(\mathrm{~m}, 2 \mathrm{H}), 3.42(\mathrm{~m}, 2 \mathrm{H})$, 2.97-2.92 (m, 1H), 2.51-2.39 (m, 1H), 2.34-2.23 (m, 1H), $2.27(\mathrm{t}, J=7.8 \mathrm{~Hz}, 2 \mathrm{H}), 1.66-1.56(\mathrm{~m}, 5 \mathrm{H})$, $1.55-1.48(\mathrm{~m}, 1 \mathrm{H}), 1.38-1.26(\mathrm{~m}, 8 \mathrm{H})$ ppm. ${ }^{13} \mathbf{C}$ NMR $\left(126 \mathrm{MHz}, \mathrm{CDCl}_{3}\right): \delta 171.9,133.5,132.4,129.2$, $127.0,126.3\left(\mathrm{q}, J_{C-F}=278.1 \mathrm{~Hz}\right), 125.5,125.4,95.2,81.2,67.0,66.7,46.1,41.9,39.1\left(\mathrm{q}, J_{C-F}=27.8 \mathrm{~Hz}\right)$, 34.8, 33.1, 29.5, 29.4, 29.4, 29.2, 26.9, 26.7 (q, $\left.J_{C-F}=3.0 \mathrm{~Hz}\right), 25.3$ ppm. ${ }^{19} \mathbf{F}$ NMR $\left(470 \mathrm{MHz}, \mathrm{CDCl}_{3}\right)$ : 
$\delta-65.15(\mathrm{t}, J=10.6 \mathrm{~Hz}, 3 \mathrm{~F}) . \mathbf{H R M S} \mathrm{m} / \mathrm{z}(\mathrm{ESI})$ : calcd. for $\mathrm{C}_{24} \mathrm{H}_{31} \mathrm{BrF}_{3} \mathrm{NO}_{2} \mathrm{Na}[\mathrm{M}+\mathrm{Na}]^{+}: 476.2383$; found: 476.2383.

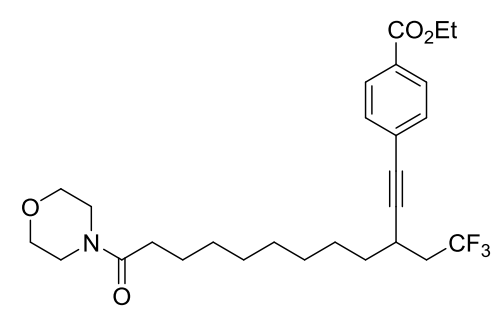

3aq: 12-(4-chlorophenyl)-1-morpholino-10-(2,2,2-trifluoroethyl)dodec-11-yn-1-one. Prepared according to the general procedure. Reaction was run using $1 \mathbf{y}(50.6 \mathrm{mg}, 0.2 \mathrm{mmol}), \mathrm{NaOAc}(3.3 \mathrm{mg}$, $0.04 \mathrm{mmol}), \mathrm{AgOCOCF}_{3}(4.4 \mathrm{mg}, 0.02 \mathrm{mmol}), \mathrm{BIOMe}(22.2 \mathrm{mg}, 0.08 \mathrm{mmol}), \mathrm{Me}_{3} \mathrm{SiCF}_{3}(120 \mu \mathrm{L}, 0.8$ $\mathrm{mmol}), 2 \mathrm{~g}(252.0 \mathrm{mg}, 0.6 \mathrm{mmol})$ and DMSO $(1.0 \mathrm{~mL})$. The product was purified by flash column chromatography on silica gel (hexane/EtOAc) and obtained a colorless oil (0.18 mmol, $91.1 \mathrm{mg}, 92 \%)$, $\mathrm{R}_{\mathrm{f}}=0.3$ (hexane : Acetone $\left.=2: 1\right) .{ }^{1} \mathbf{H}$ NMR $\left(400 \mathrm{MHz}, \mathrm{CDCl}_{3}\right): \delta 7.91(\mathrm{~d}, J=8.4 \mathrm{~Hz}, 2 \mathrm{H}), 7.39(\mathrm{~d}, J=$ $8.4 \mathrm{~Hz}, 2 \mathrm{H}), 4.30$ (q, J=7.2 Hz, 2H), $3.59(\mathrm{~m}, 4 \mathrm{H}), 3.56(\mathrm{~m}, 2 \mathrm{H}), 3.38(\mathrm{~m}, 2 \mathrm{H}), 2.92-2.84(\mathrm{~m}, 1 \mathrm{H}), 2.43-$ $2.31(\mathrm{~m}, 1 \mathrm{H}), 2.30-2.17(\mathrm{~m}, 1 \mathrm{H}), 2.24(\mathrm{t}, J=7.6 \mathrm{~Hz}, 2 \mathrm{H}), 1.61-1.49(\mathrm{~m}, 5 \mathrm{H}), 1.45-1.38(\mathrm{~m}, 1 \mathrm{H}), 1.33(\mathrm{t}$, $J=7.2 \mathrm{~Hz}, 3 \mathrm{H}), 1.30-1.23(\mathrm{~m}, 8 \mathrm{H}) \mathrm{ppm} .{ }^{13} \mathbf{C} \mathbf{N M R}\left(101 \mathrm{MHz}, \mathrm{CDCl}_{3}\right): \delta 171.8,166.0,131.4,129.6$, $129.3,127.9,126.2$ (q, $\left.J_{C-F}=278.6 \mathrm{~Hz}\right), 93.3,82.0,66.9,66.6,61.1,46.0,41.8,38.9$ (q, $J_{C-F}=27.8 \mathrm{~Hz}$ ), 34.6, 33.0, 29.4, 29.3, 29.3, 29.1, 26.9, 26.5 (q, $\left.J_{C-F}=2.7 \mathrm{~Hz}\right), 25.2,14.2$ ppm. ${ }^{19} \mathbf{F}$ NMR (470 MHz, $\left.\mathrm{CDCl}_{3}\right): \delta-65.19(\mathrm{t}, J=10.6 \mathrm{~Hz}, 3 \mathrm{~F})$. HRMS m/z (ESI): calcd. for $\mathrm{C}_{27} \mathrm{H}_{36} \mathrm{~F}_{3} \mathrm{NO}_{4} \mathrm{Na}[\mathrm{M}+\mathrm{Na}]^{+}: 518.2489$; found: 518.2488 .

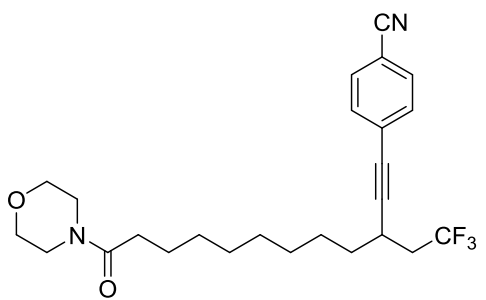

3ar: 4-(12-morpholino-12-oxo-3-(2,2,2-trifluoroethyl)dodec-1-yn-1-yl)benzonitrile. Prepared according to the general procedure. Reaction was run using $\mathbf{1 y}(50.6 \mathrm{mg}, 0.2 \mathrm{mmol}), \mathrm{NaOAc}(3.3 \mathrm{mg}$, $0.04 \mathrm{mmol}), \mathrm{AgOCOCF}_{3}(4.4 \mathrm{mg}, 0.02 \mathrm{mmol}), \mathrm{BIOMe}(22.2 \mathrm{mg}, 0.08 \mathrm{mmol}), \mathrm{Me}_{3} \mathrm{SiCF}_{3}(120 \mu \mathrm{L}, 0.8$ $\mathrm{mmol}), \mathbf{2 h}(252.0 \mathrm{mg}, 0.6 \mathrm{mmol})$ and DMSO $(1.0 \mathrm{~mL})$. The product was purified by flash column chromatography on silica gel (hexane/EtOAc) and obtained a colorless oil (0.16 mmol, $71.7 \mathrm{mg}, 80 \%)$, $\mathrm{R}_{\mathrm{f}}=0.3$ (hexane : Acetone $\left.=2: 1\right) .{ }^{1} \mathbf{H}$ NMR $\left(400 \mathrm{MHz}, \mathrm{CDCl}_{3}\right): \delta 7.54(\mathrm{~d}, J=8.0 \mathrm{~Hz}, 2 \mathrm{H}), 7.42(\mathrm{~d}, J=$ $8.0 \mathrm{~Hz}, 2 \mathrm{H}), 3.62(\mathrm{~m}, 4 \mathrm{H}), 3.56(\mathrm{~m}, 2 \mathrm{H}), 3.41(\mathrm{~m}, 2 \mathrm{H}), 2.93-2.86(\mathrm{~m}, 1 \mathrm{H}), 2.45-2.32(\mathrm{~m}, 1 \mathrm{H}), 2.30-2.21$ $(\mathrm{m}, 1 \mathrm{H}), 2.26(\mathrm{t}, J=7.6 \mathrm{~Hz}, 2 \mathrm{H}), 1.62-1.48(\mathrm{~m}, 5 \mathrm{H}), 1.46-1.37(\mathrm{~m}, 1 \mathrm{H}), 1.33-1.25(\mathrm{~m}, 8 \mathrm{H}) \mathrm{ppm} .{ }^{13} \mathrm{C}$ NMR (101 MHz, $\left.\mathrm{CDCl}_{3}\right): \delta 171.8,132.2,131.9,128.3,126.1$ (q, $\left.J_{C-F}=278.5 \mathrm{~Hz}\right), 118.5,111.2,95.0$, 81.3, 67.0, 66.6, 46.0, 41.8, 39.0 (q, $J_{C-F}=28.0 \mathrm{~Hz}$ ), 34.5, 33.0, 29.4, 29.3, 29.3, 29.1, 26.9, 26.5 (q, $J_{C-}$ $\left.{ }_{F}=2.9 \mathrm{~Hz}\right), 25.2 \mathrm{ppm} .{ }^{19} \mathbf{F}$ NMR $\left(470 \mathrm{MHz}, \mathrm{CDCl}_{3}\right): \delta-65.22$ (t, $\left.J=10.6 \mathrm{~Hz}, 3 \mathrm{~F}\right) . \mathbf{H R M S ~ m} / \mathrm{z}(\mathrm{ESI})$ : calcd. for $\mathrm{C}_{25} \mathrm{H}_{31} \mathrm{~F}_{3} \mathrm{~N}_{2} \mathrm{O}_{2} \mathrm{Na}[\mathrm{M}+\mathrm{Na}]^{+}$: 471.2230; found: 471.2230 . 


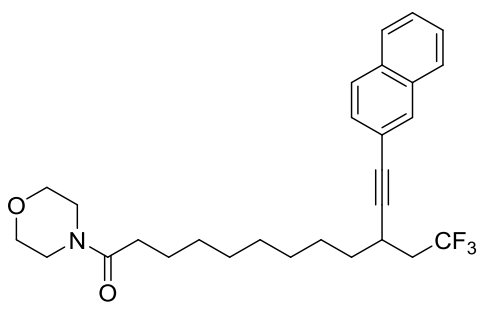

3as: 1-morpholino-12-(naphthalen-2-yl)-10-(2,2,2-trifluoroethyl)dodec-11-yn-1-one. Prepared according to the general procedure. Reaction was run using $1 \mathbf{y}(50.6 \mathrm{mg}, 0.2 \mathrm{mmol}), \mathrm{NaOAc}(3.3 \mathrm{mg}$, $0.04 \mathrm{mmol}), \mathrm{AgOCOCF}_{3}(4.4 \mathrm{mg}, 0.02 \mathrm{mmol}), \mathrm{BIOMe}(22.2 \mathrm{mg}, 0.08 \mathrm{mmol}), \mathrm{Me}_{3} \mathrm{SiCF}_{3}(120 \mu \mathrm{L}, 0.8$ $\mathrm{mmol}), 2 \mathbf{i}(238.8 \mathrm{mg}, 0.6 \mathrm{mmol})$ and DMSO $(1.0 \mathrm{~mL})$. The product was purified by flash column chromatography on silica gel (hexane/EtOAc) and obtained a colorless oil (0.08 mmol, $38.8 \mathrm{mg}, 41 \%)$, $\mathrm{R}_{\mathrm{f}}=0.3$ (hexane : Acetone $\left.=3: 1\right) .{ }^{1} \mathbf{H}$ NMR $\left(500 \mathrm{MHz}, \mathrm{CDCl}_{3}\right): \delta 7.91(\mathrm{~s}, 1 \mathrm{H}), 7.80-7.74(\mathrm{~m}, 3 \mathrm{H}), 7.48$ $7.43(\mathrm{~m}, 3 \mathrm{H}), 3.63(\mathrm{~m}, 4 \mathrm{H}), 3.60(\mathrm{~m}, 2 \mathrm{H}), 3.41$ (m, 2H), 3.00-2.94 (m, 1H), 2.52-2.41 (m, 1H), 2.37-2.27 $(\mathrm{m}, 1 \mathrm{H}), 2.28(\mathrm{t}, J=7.8 \mathrm{~Hz}, 2 \mathrm{H}), 1.67-1.59(\mathrm{~m}, 5 \mathrm{H}), 1.54-1.46(\mathrm{~m}, 1 \mathrm{H}), 1.39-1.30(\mathrm{~m}, 8 \mathrm{H}) \mathrm{ppm} .{ }^{13} \mathrm{C}$ NMR (126 MHz, $\left.\mathrm{CDCl}_{3}\right): \delta 171.9,133.1,132.7,131.3,128.7,128.0,127.8,127.7,126.6,126.4\left(\mathrm{q}, J_{C-F}\right.$ $=278.1 \mathrm{~Hz}), 120.8,90.6,83.0,67.0,66.7,46.1,41.9,39.3\left(\mathrm{q}, J_{C-F}=27.6 \mathrm{~Hz}\right), 34.9,33.2,29.5,29.4$, 29.4, 29.3, 27.0, 26.6 (q, $J_{C-F}=2.9 \mathrm{~Hz}$ ), 25.3 (one carbon missing due to overlap) ppm. ${ }^{19} \mathbf{F}$ NMR (470 $\mathrm{MHz}, \mathrm{CDCl}_{3}$ ): $\delta-65.07$ (t, $\left.J=10.8 \mathrm{~Hz}, 3 \mathrm{~F}\right) . \mathbf{H R M S} \mathrm{m} / \mathrm{z}(\mathrm{ESI})$ : calcd. for $\mathrm{C}_{28} \mathrm{H}_{34} \mathrm{~F}_{3} \mathrm{NO}_{2} \mathrm{Na}[\mathrm{M}+\mathrm{Na}]^{+}$: 496.2434; found: 496.2434 .<smiles>O=C(CCCCCCCCCC(C#C[In])CC(F)(F)F)N1CCOCC1</smiles>

3at: 1-morpholino-10-(2,2,2-trifluoroethyl)-12-(triisopropylsilyl)dodec-11-yn-1-one. Prepared according to the general procedure. Reaction was run using $1 \mathbf{y}(50.6 \mathrm{mg}, 0.2 \mathrm{mmol}), \mathrm{NaOAc}(3.3 \mathrm{mg}$, $0.04 \mathrm{mmol}), \mathrm{AgOCOCF}_{3}(4.4 \mathrm{mg}, 0.02 \mathrm{mmol}), \mathrm{BIOMe}(22.2 \mathrm{mg}, 0.08 \mathrm{mmol}), \mathrm{Me}_{3} \mathrm{SiCF}_{3}(120 \mu \mathrm{L}, 0.8$ $\mathrm{mmol}), 2 \mathbf{j}(257 \mathrm{mg}, 0.6 \mathrm{mmol})$ and DMSO $(1.0 \mathrm{~mL})$. The product was purified by flash column chromatography on silica gel (hexane/EtOAc) and obtained a colorless oil (0.07 mmol, $35.2 \mathrm{mg}, 35 \%)$, $\mathrm{R}_{\mathrm{f}}=0.4$ (hexane : EtOAc = $\left.1.5: 1\right) .{ }^{1} \mathbf{H}$ NMR $\left(400 \mathrm{MHz}, \mathrm{CDCl}_{3}\right): \delta 3.64(\mathrm{~m}, 4 \mathrm{H}), 3.60(\mathrm{~m}, 2 \mathrm{H}), 3.44(\mathrm{~m}$, $2 \mathrm{H}), 2.75-2.68(\mathrm{~m}, 1 \mathrm{H}), 2.38-2.25(\mathrm{~m}, 1 \mathrm{H}), 2.29(\mathrm{t}, J=7.6 \mathrm{~Hz}, 2 \mathrm{H}), 2.23-2.11(\mathrm{~m}, 1 \mathrm{H}), 1.65-1.39(\mathrm{~m}$, $6 \mathrm{H}), 1.34-1.25(\mathrm{~m}, 8 \mathrm{H}), 1.09-0.98(\mathrm{~m}, 21 \mathrm{H}) \mathrm{ppm} .{ }^{13} \mathbf{C} \mathbf{N M R}\left(101 \mathrm{MHz}, \mathrm{CDCl}_{3}\right): \delta 172.0,126.3\left(\mathrm{q}, J_{C-F}\right.$ $=278.6 \mathrm{~Hz}$ ), 108.8, 82.6, 67.1, 66.8, 46.1, 42.0, 39.4 (q, $\left.J_{C-F}=27.7 \mathrm{~Hz}\right), 34.9,33.2,29.6,29.5,29.4$, 29.2, 26.9, 26.8, 25.3, 18.6, 11.3 ppm. ${ }^{19} \mathbf{F ~ N M R}\left(470 \mathrm{MHz}, \mathrm{CDCl}_{3}\right): \delta-65.10(\mathrm{t}, J=10.6 \mathrm{~Hz}, 3 \mathrm{~F})$. HRMS $\mathrm{m} / \mathrm{z}$ (ESI): calcd. for $\mathrm{C}_{27} \mathrm{H}_{48} \mathrm{~F}_{3} \mathrm{NO}_{2} \mathrm{Si} \mathrm{Na}[\mathrm{M}+\mathrm{Na}]^{+}: 526.3299$; found: 526.3298 . 
Spectra.

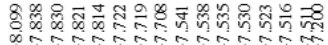 \\ ifis}

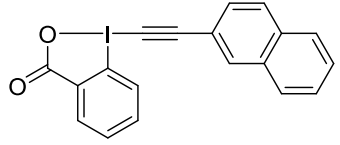

$2 \mathrm{i}\left(\mathrm{CDCl}_{3}, 500 \mathrm{MHz}\right)$

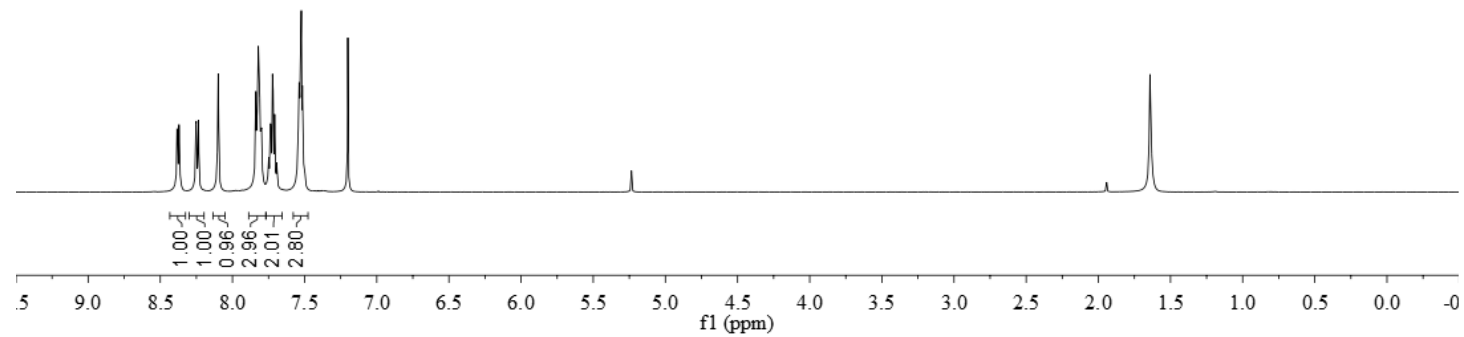

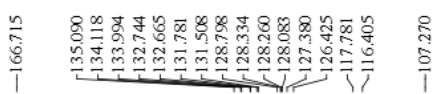

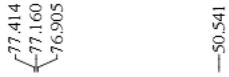

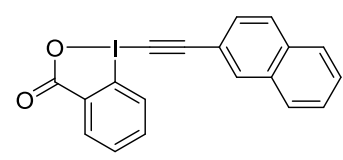

$2 \mathrm{i}\left(\mathrm{CDCl}_{3}, 126 \mathrm{MHz}\right)$

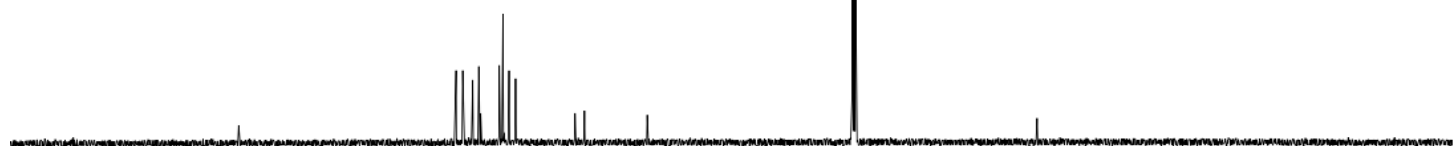

$\begin{array}{lllllllllll}10 & 190 & 180 & 170 & 160 & 150 & 140 & 130 & 120 & 110 & \begin{array}{c}100 \\ \text { f1 (ppm) }\end{array}\end{array}$ 


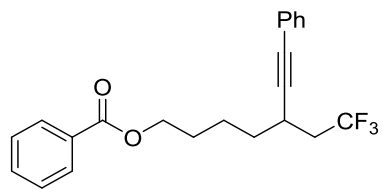

3a $\left(\mathrm{CDCl}_{3}, 400 \mathrm{MHz}\right)$
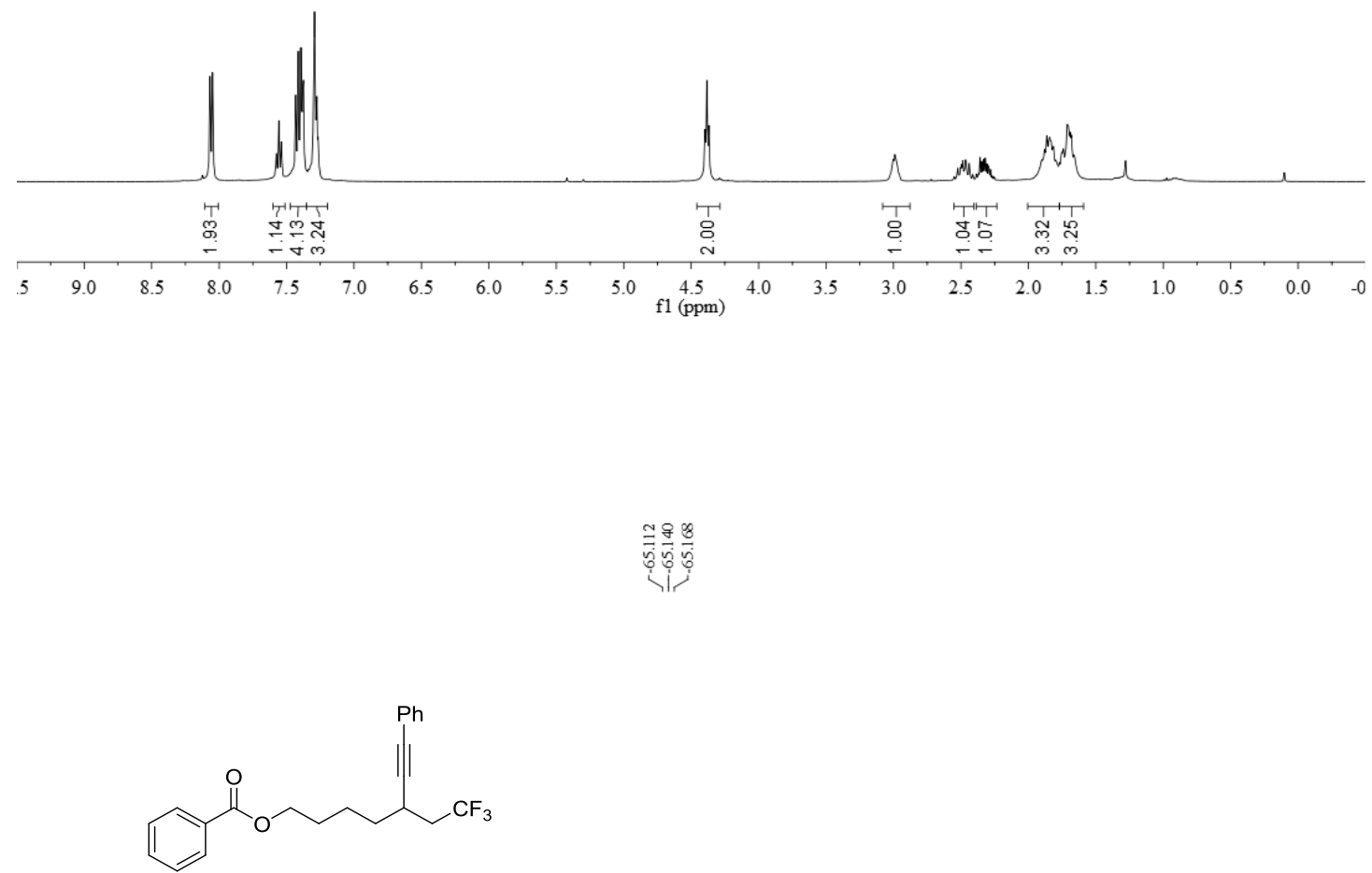

3a $\left(\mathrm{CDCl}_{3}, 376 \mathrm{MHz}\right)$

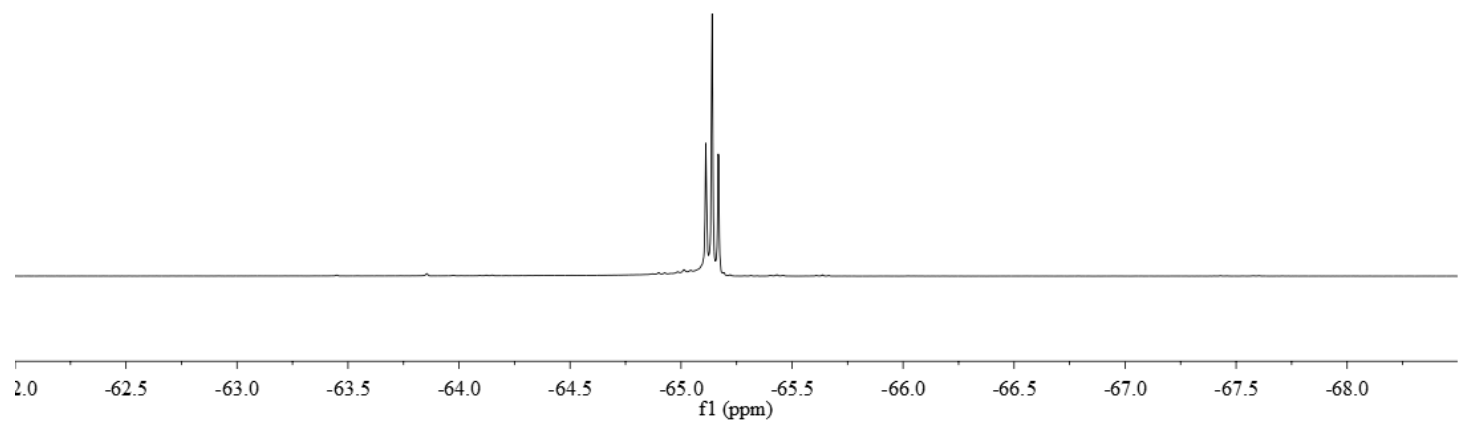




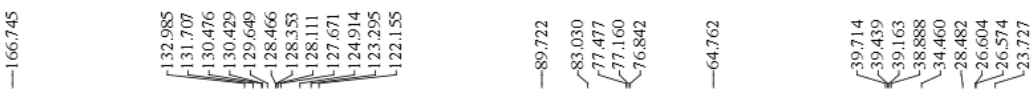

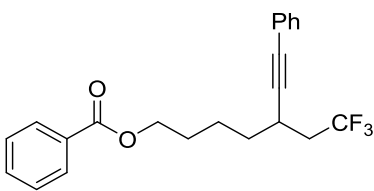

3a $\left(\mathrm{CDCl}_{3}, 101 \mathrm{MHz}\right)$
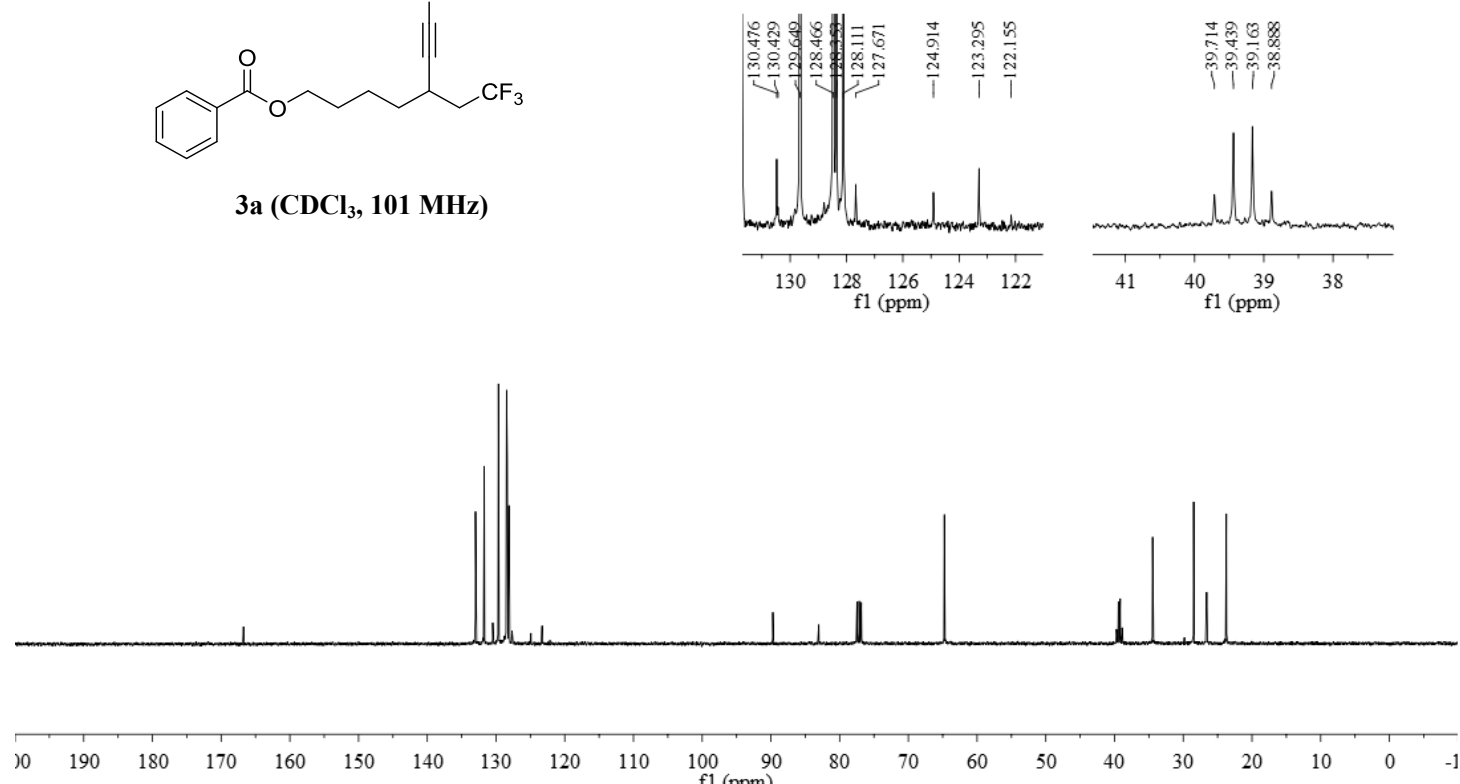

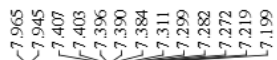

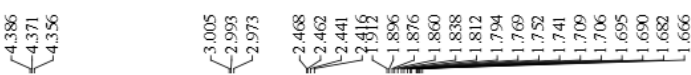<smiles>Cc1ccc(C(=O)OCCCCC(C#Cc2ccccc2)CC(F)(F)F)cc1</smiles>

$3 \mathrm{~b}\left(\mathrm{CDCl}_{3}, 400 \mathrm{MHz}\right)$

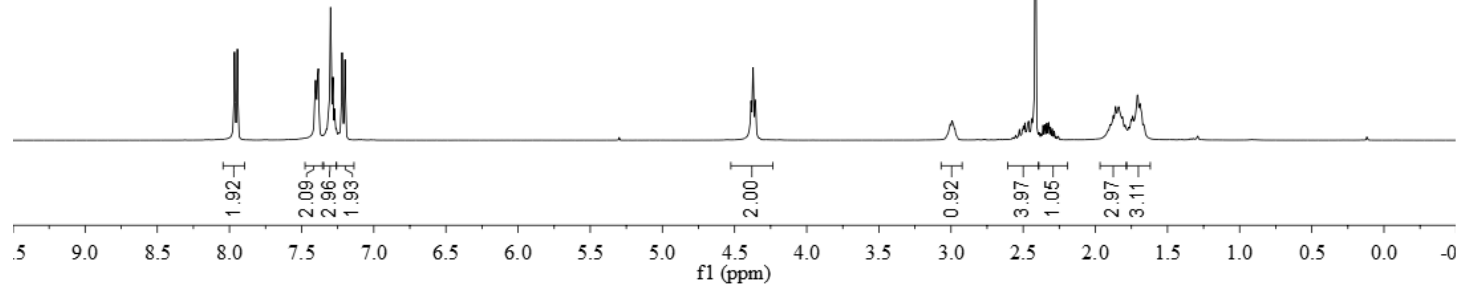




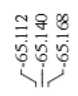
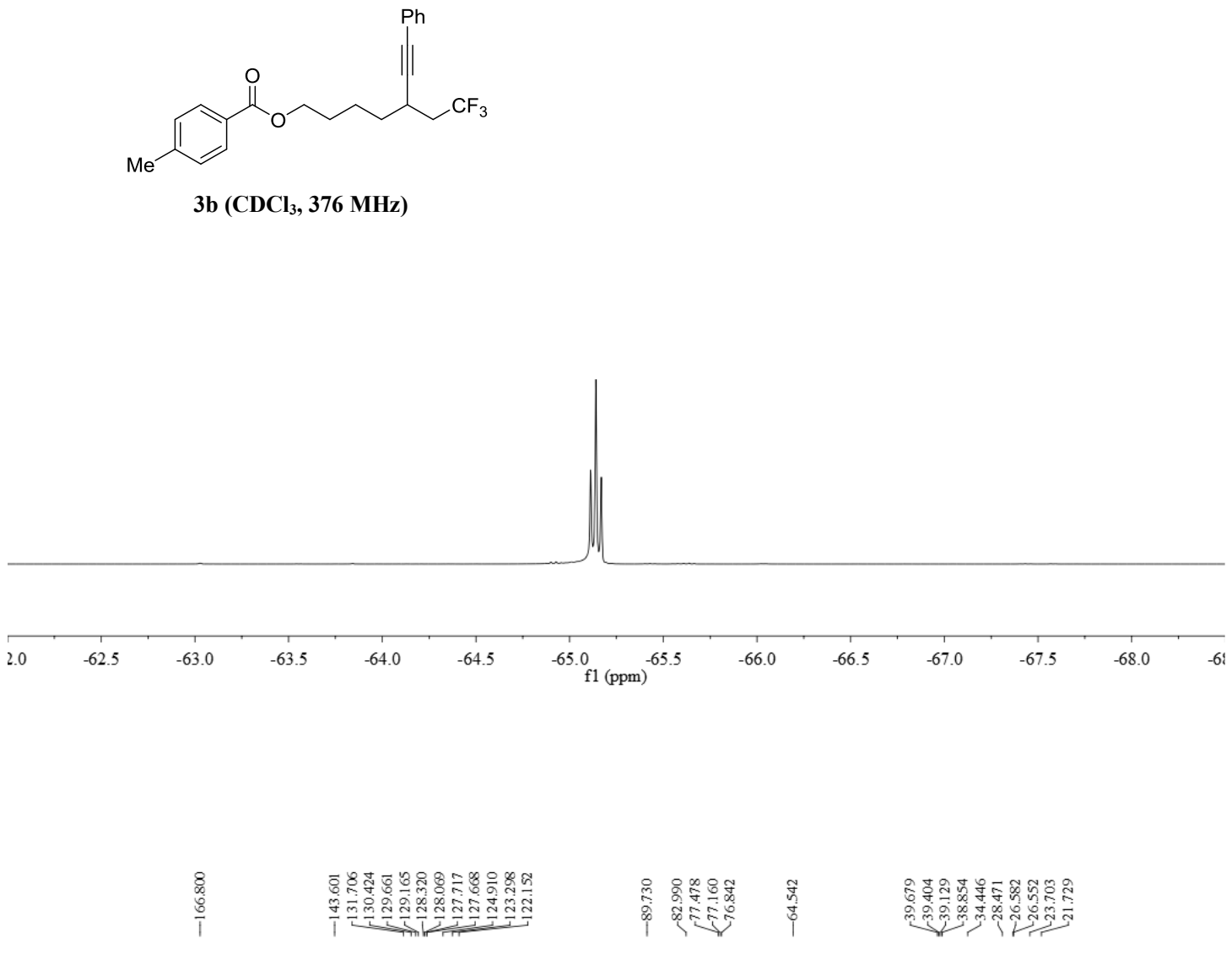

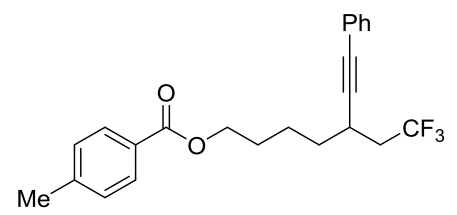

3b $\left(\mathrm{CDCl}_{3}, 101 \mathrm{MHz}\right)$
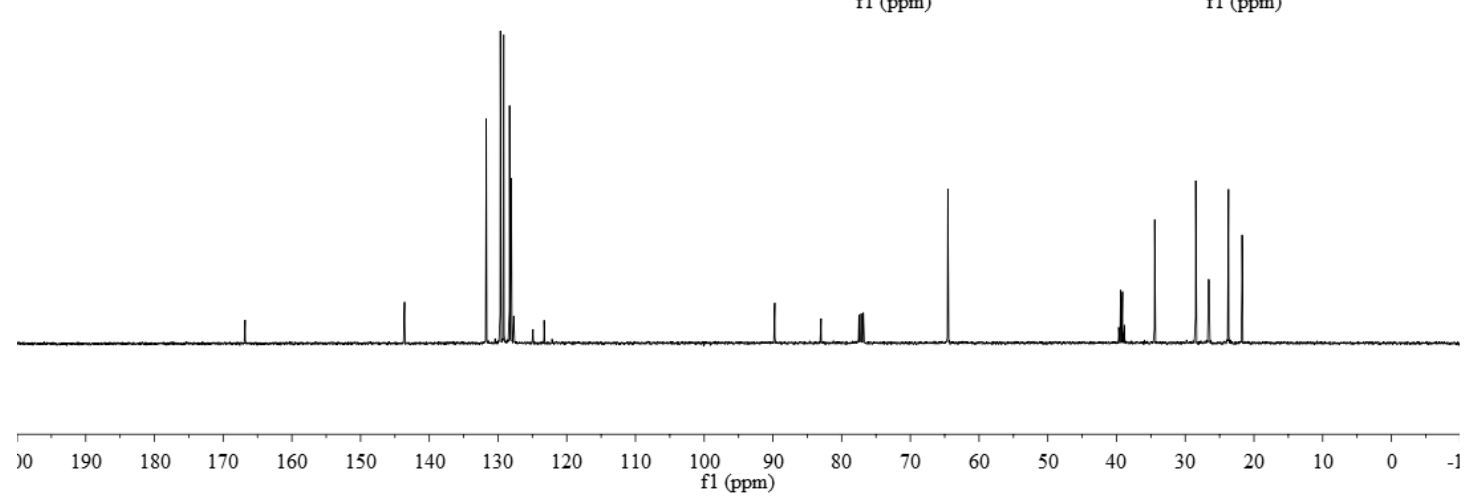

S38 

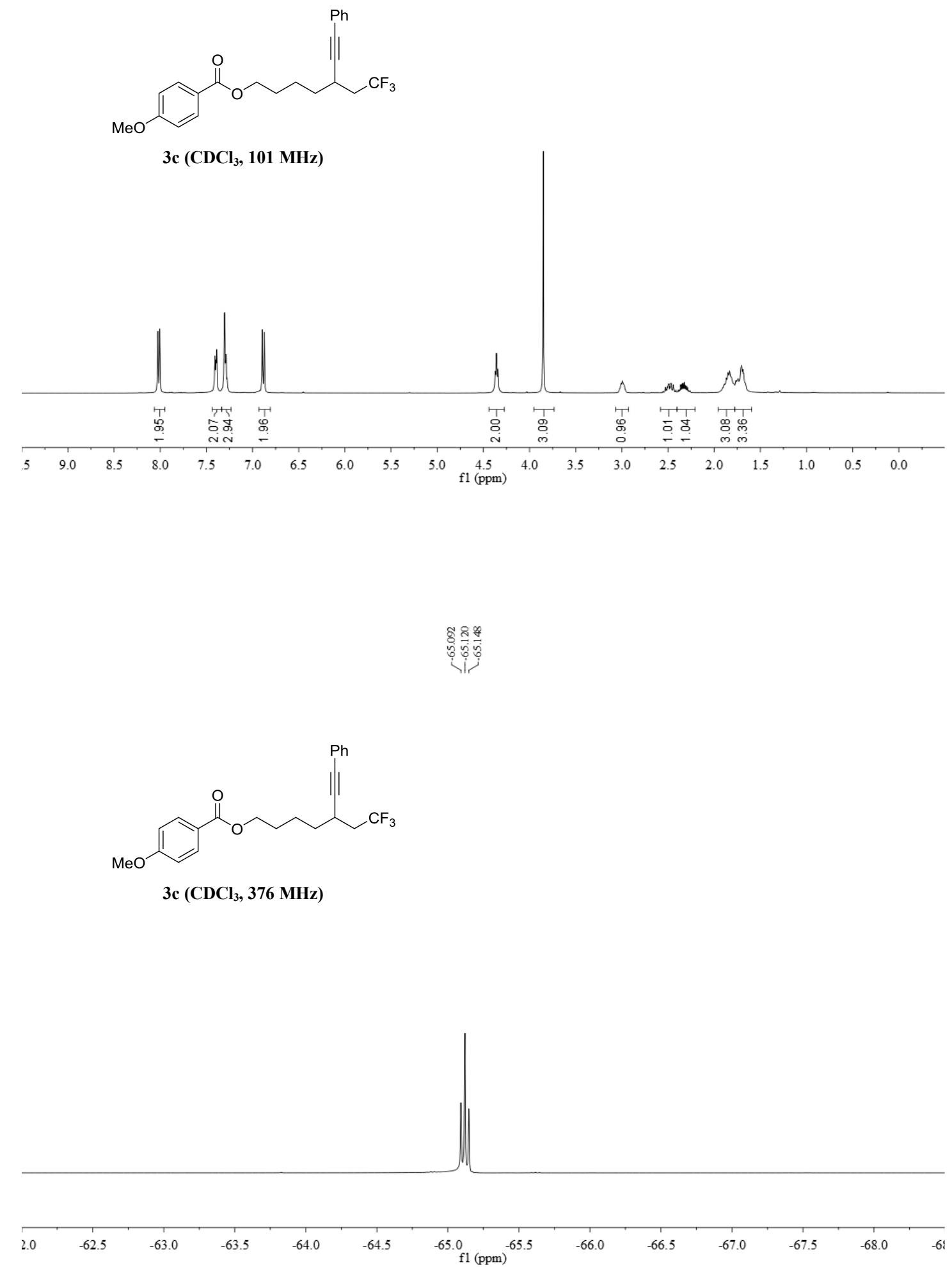
<smiles>COc1ccc(C(=O)OCCCCC(C#Cc2ccccc2)CC(F)(F)F)cc1</smiles>
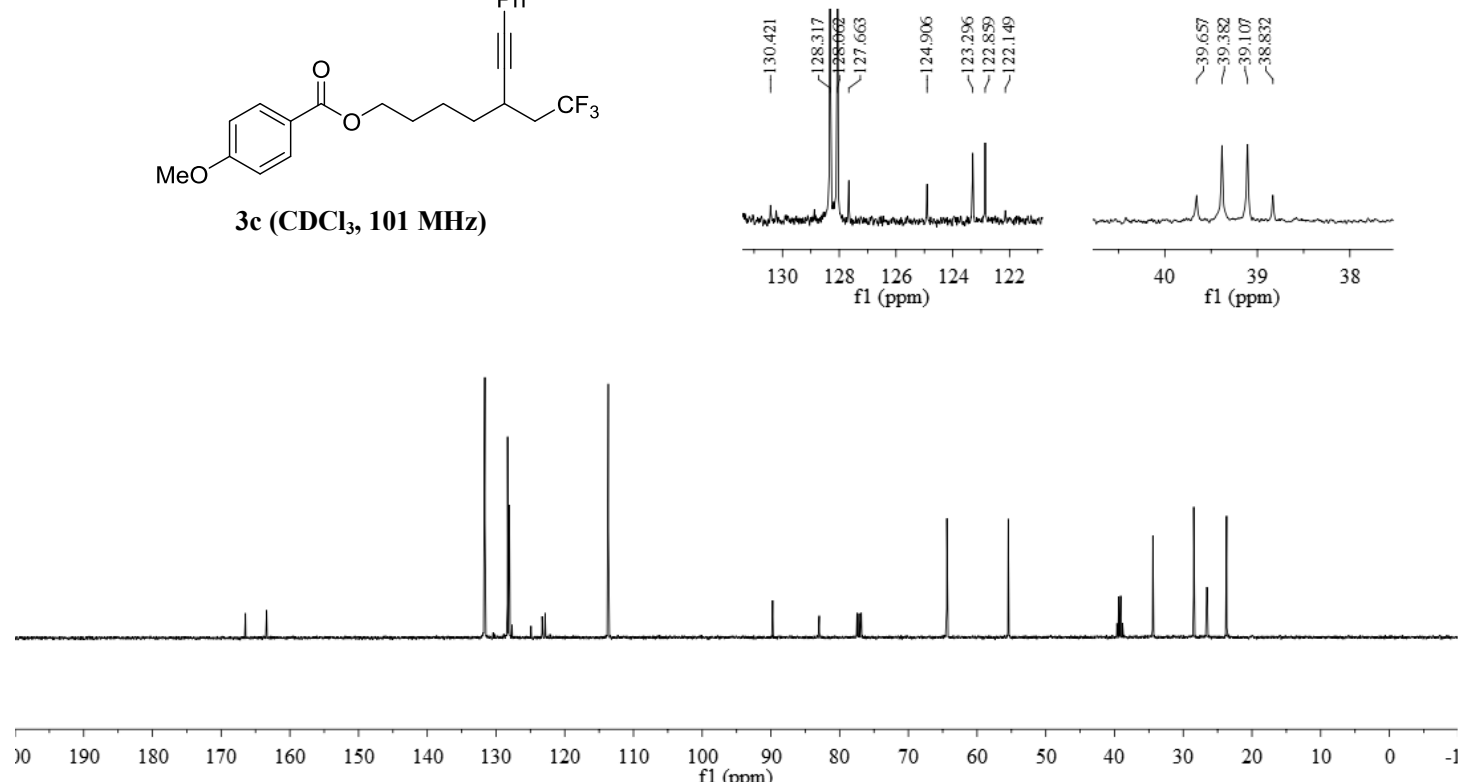

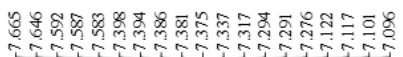

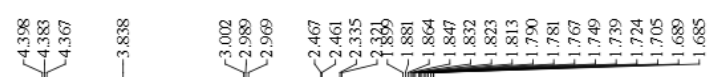

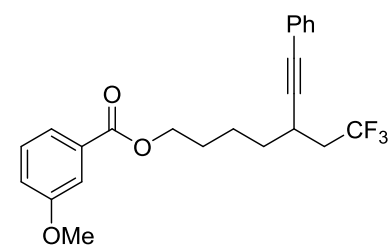

3d $\left(\mathrm{CDCl}_{3}, 400 \mathrm{MHz}\right)$

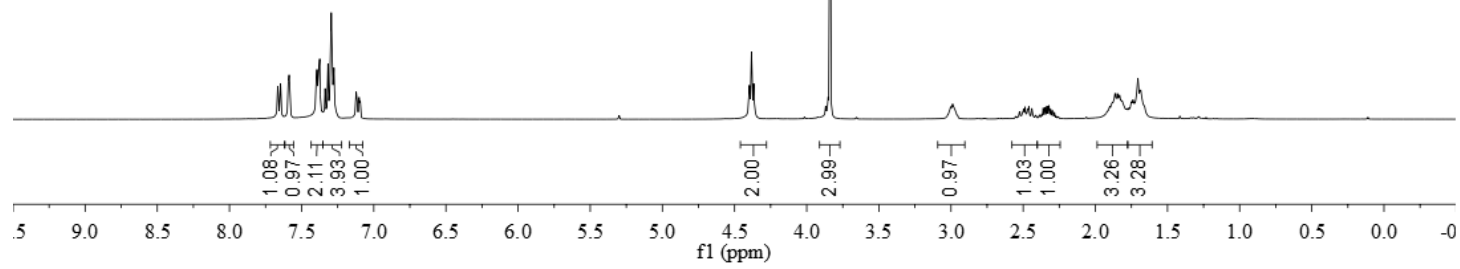




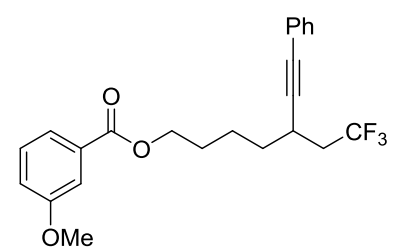

3d $\left(\mathrm{CDCl}_{3}, 376 \mathrm{MHz}\right)$

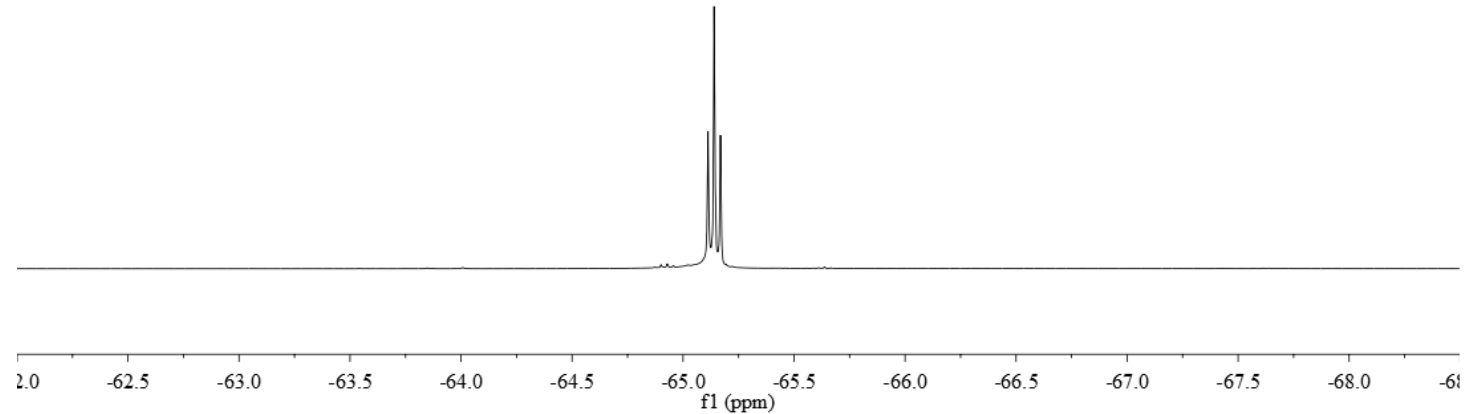

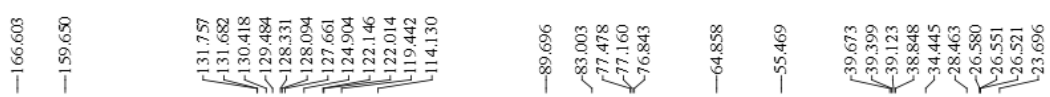<smiles>COc1cccc(C(=O)OCCCCC(C#CC(F)(F)F)CC(F)(F)F)c1</smiles>

3d $\left(\mathrm{CDCl}_{3}, 101 \mathrm{MHz}\right)$
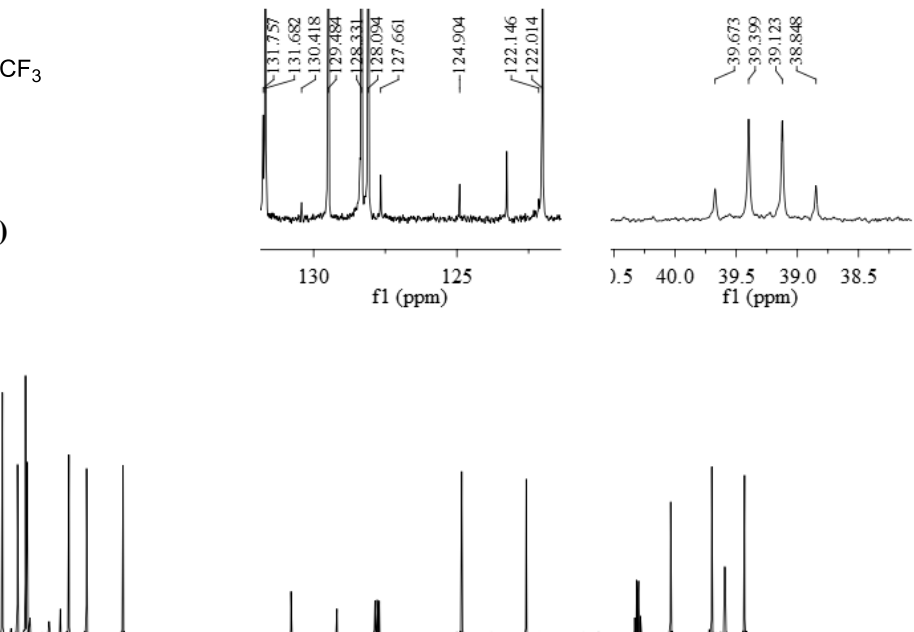

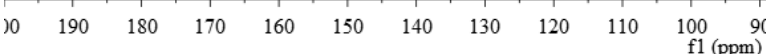




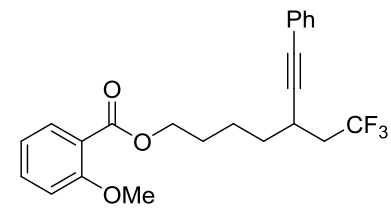

$3 \mathrm{e}\left(\mathrm{CDCl}_{3}, 400 \mathrm{MHz}\right)$

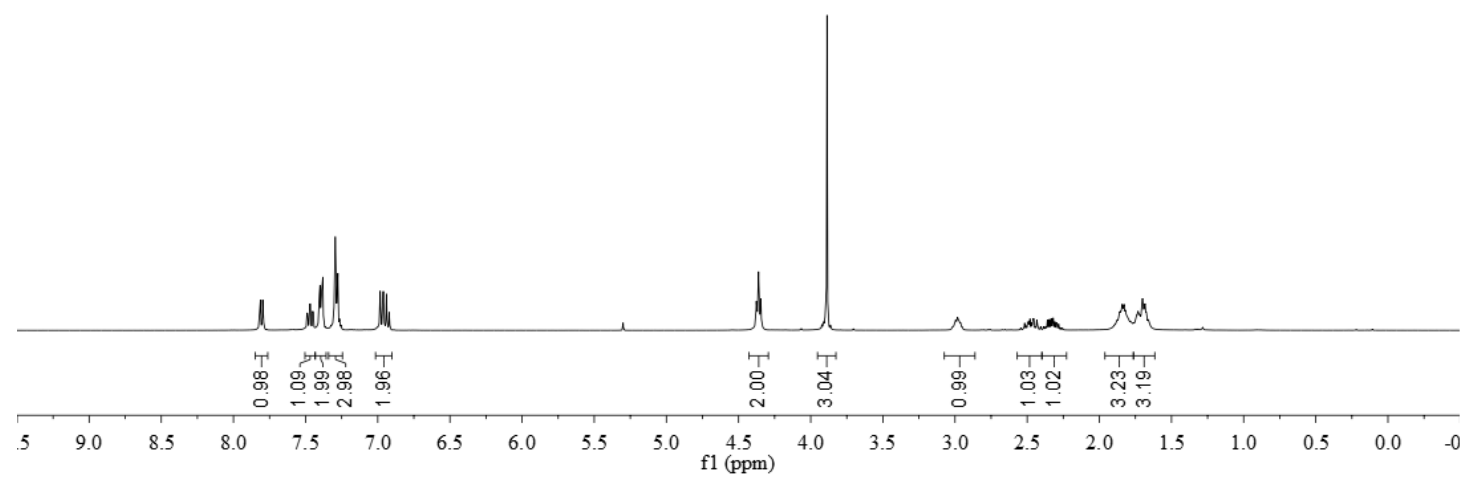

สำ<smiles>COc1ccccc1C(=O)OCCCCC(C#Cc1ccccc1)CC(F)(F)F</smiles>

$3 \mathrm{e}\left(\mathrm{CDCl}_{3}, 376 \mathrm{MHz}\right)$

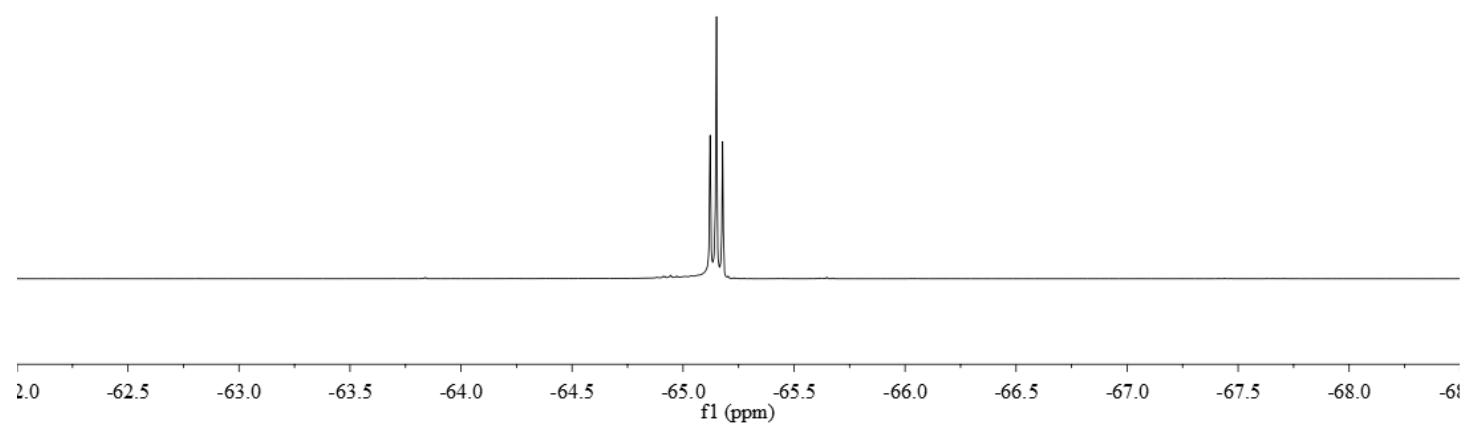




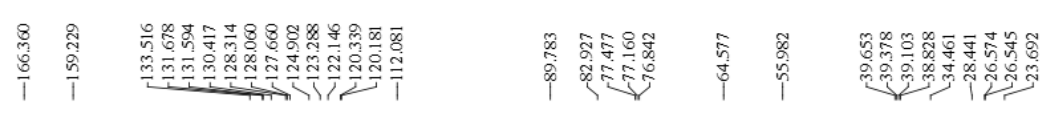
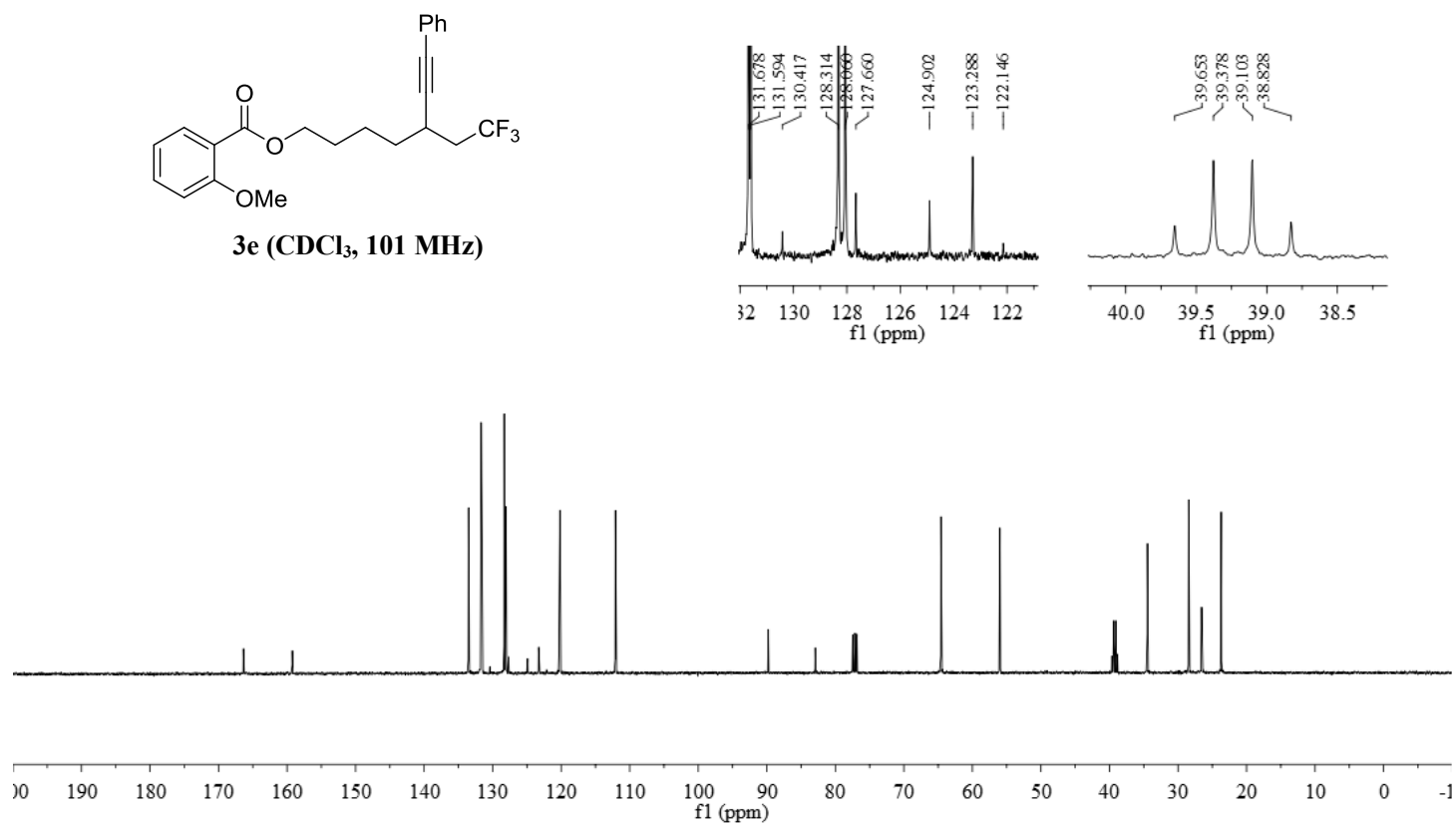

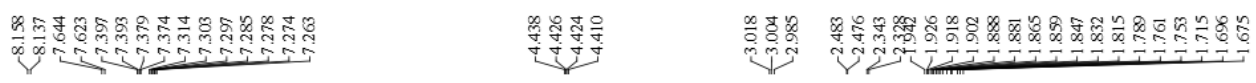<smiles>O=C(OCCCCC(C#Cc1ccccc1)CC(F)(F)F)c1ccc(C(F)(F)F)cc1</smiles>

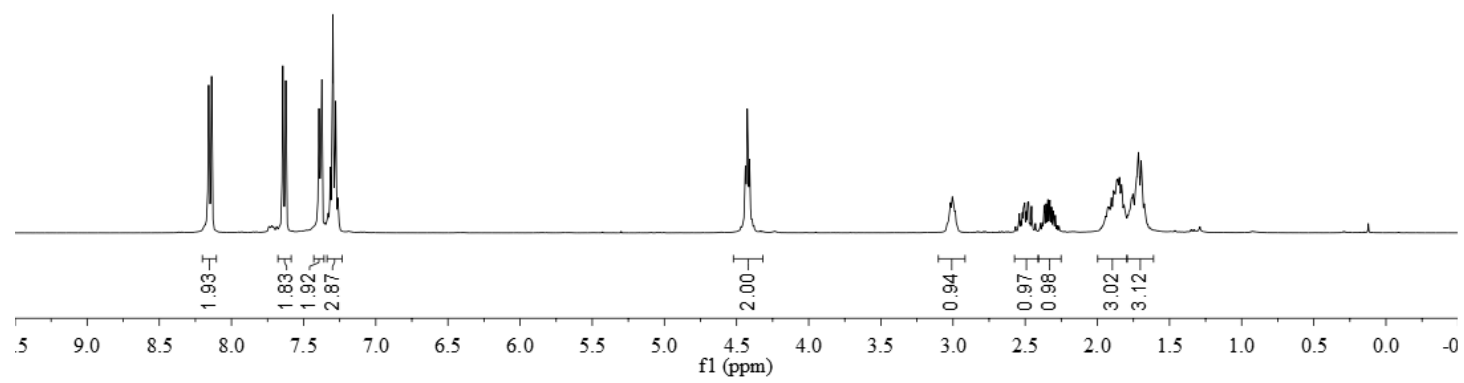


<smiles>O=C(OCCCCC(C#Cc1ccccc1)CC(F)(F)F)c1ccc(C(F)(F)F)cc1</smiles>

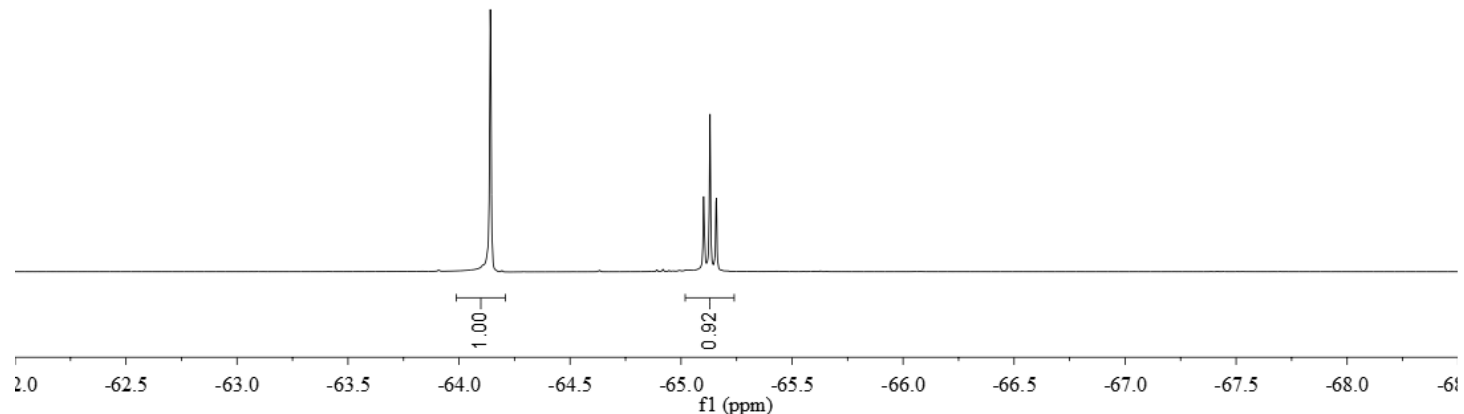

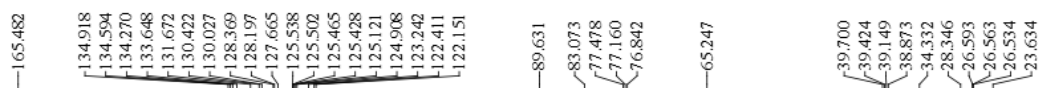

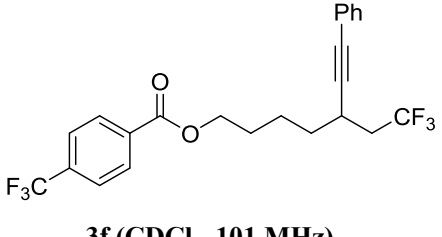
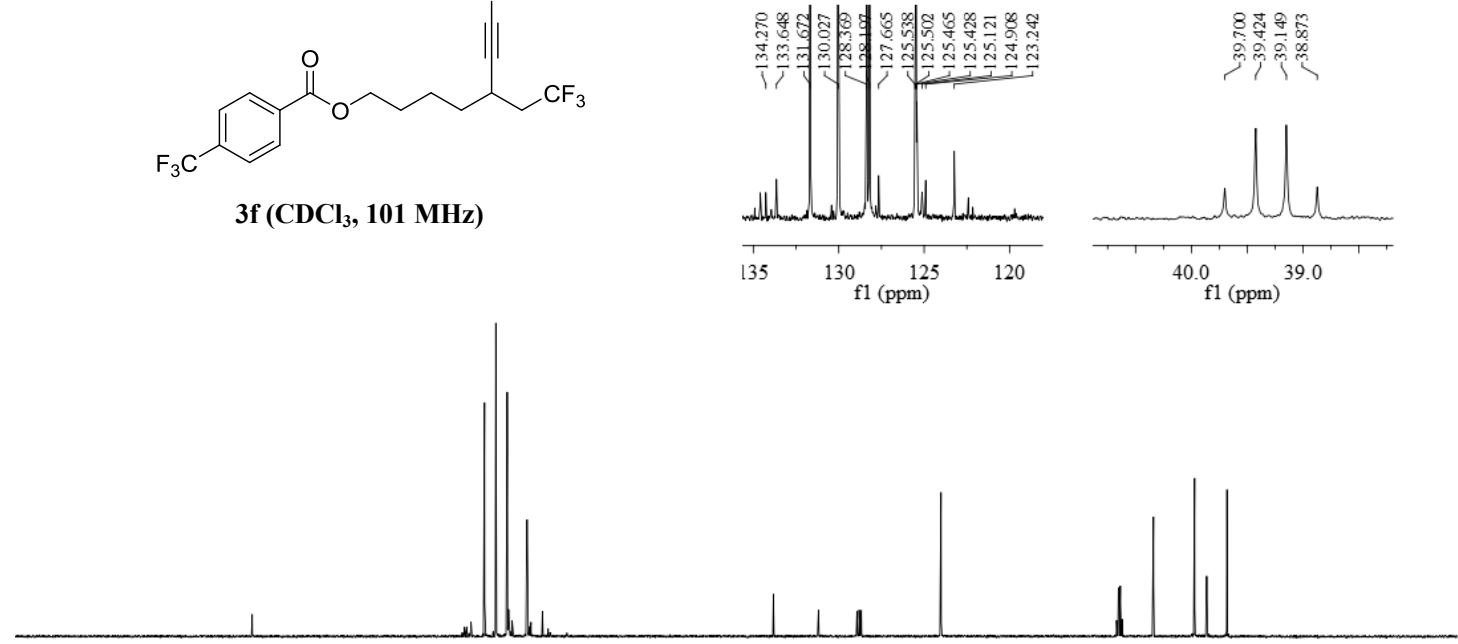

$\begin{array}{lllllllllll}10 & 190 & 180 & 170 & 160 & 150 & 140 & 130 & 120 & 110 & \begin{array}{c}100 \\ \mathrm{f} 1(\mathrm{ppm})\end{array}\end{array}$ 


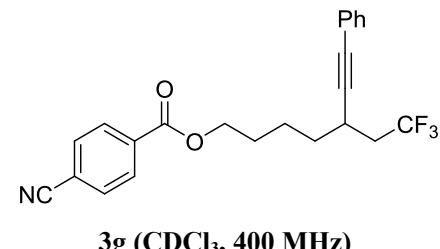

3g $\left(\mathrm{CDCl}_{3}, 400 \mathrm{MHz}\right)$
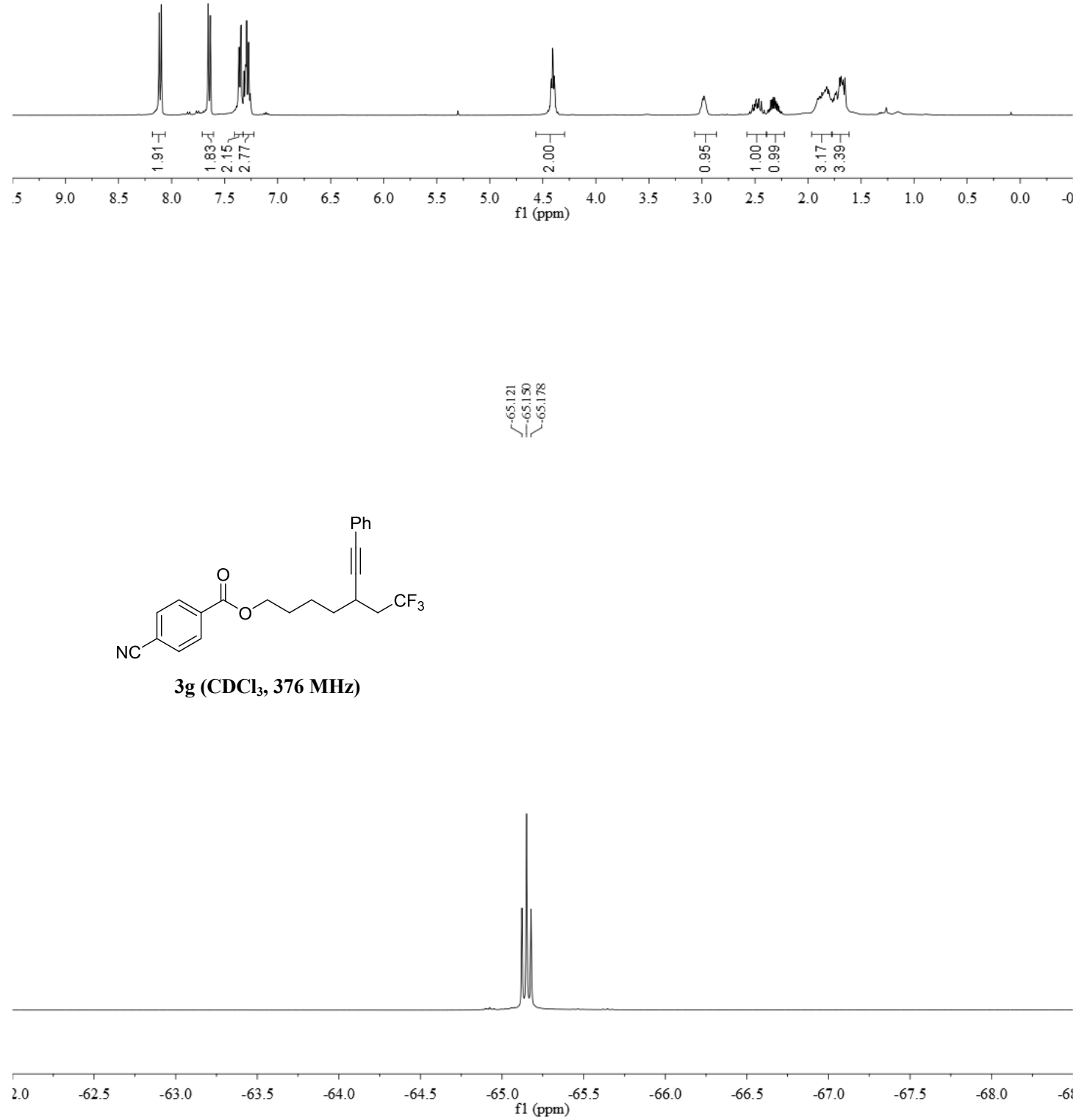


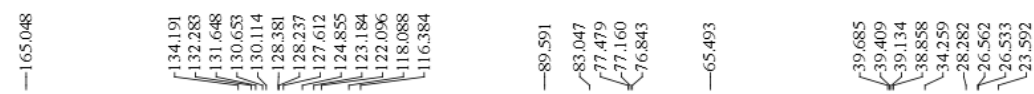
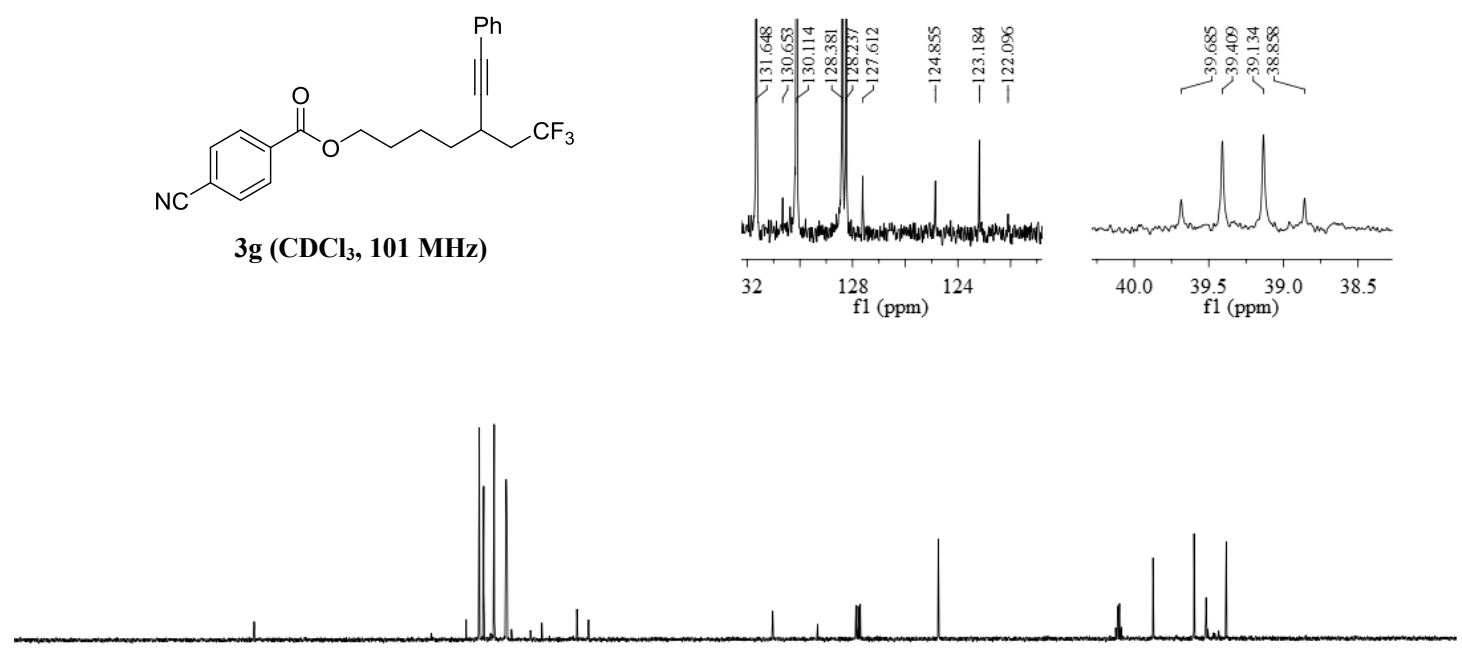

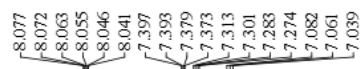

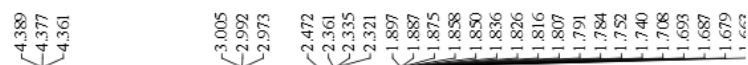<smiles>CC(C)CCCCOC(=O)c1ccc(F)cc1</smiles>

$3 \mathrm{~h}\left(\mathrm{CDCl}_{3}, 400 \mathrm{MHz}\right)$

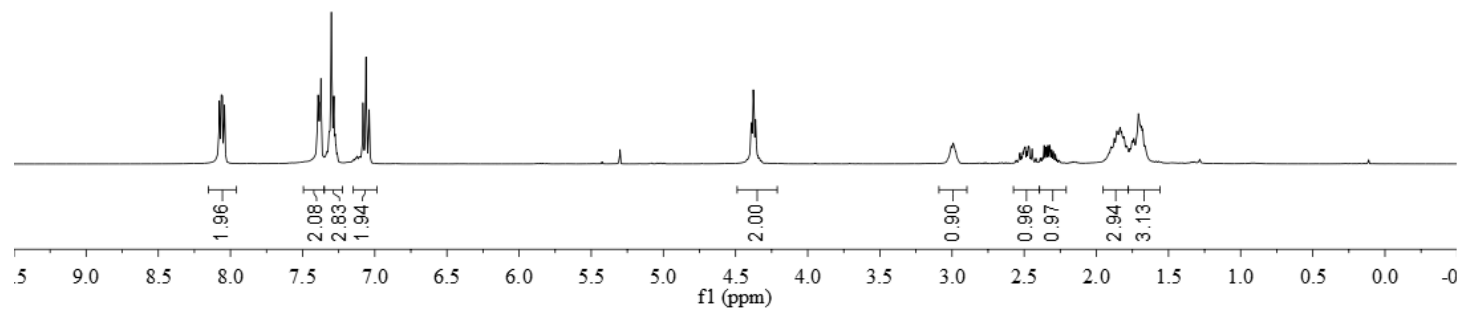


<smiles>O=C(OCCCCC(C#Cc1ccccc1)CC(F)(F)F)c1ccc(F)cc1</smiles>

$3 \mathrm{~h}\left(\mathrm{CDCl}_{3}, 376 \mathrm{MHz}\right)$

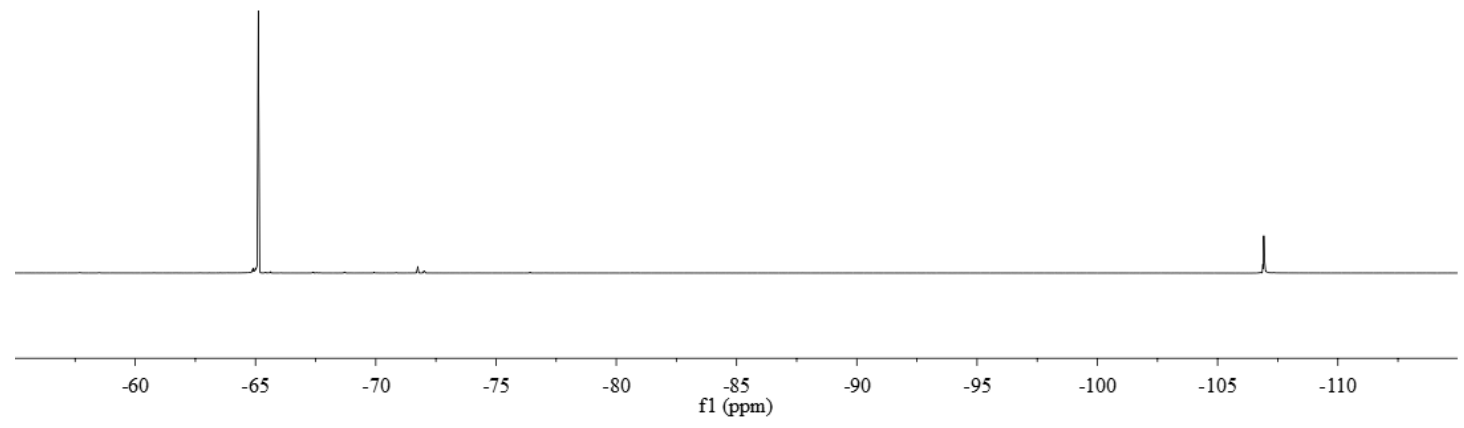

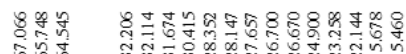

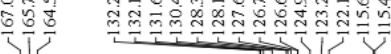

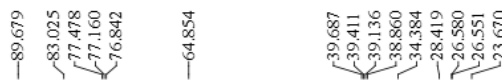<smiles>O=C(OCCCCC(C#Cc1ccccc1)CC(F)(F)F)c1ccc(F)cc1</smiles>

3h $\left(\mathrm{CDCl}_{3}, 101 \mathrm{MHz}\right)$
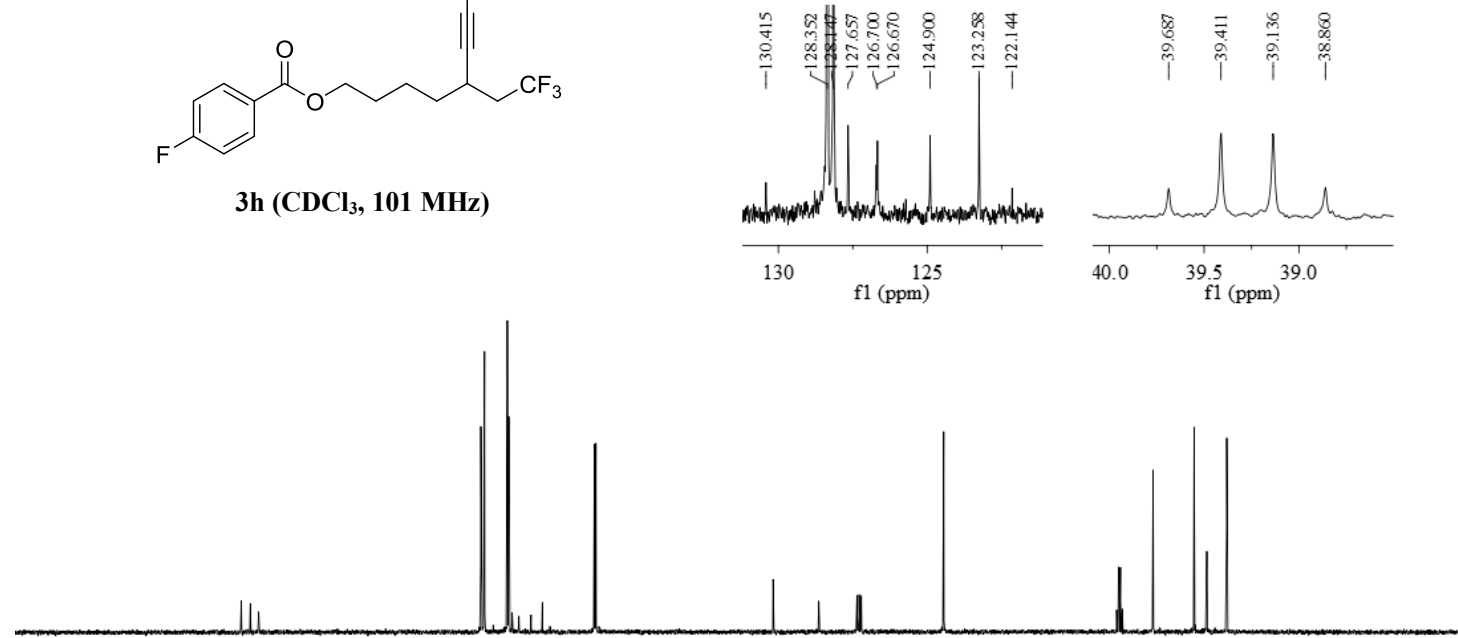

$\begin{array}{lllllllllll}10 & 190 & 180 & 170 & 160 & 150 & 140 & 130 & 120 & 110 & \begin{array}{c}100 \\ \mathrm{f} 1(\mathrm{ppm})\end{array}\end{array}$ 
<smiles>O=C(OCCCCC(C#Cc1ccccc1)CC(F)(F)F)c1ccc(Cl)cc1</smiles>

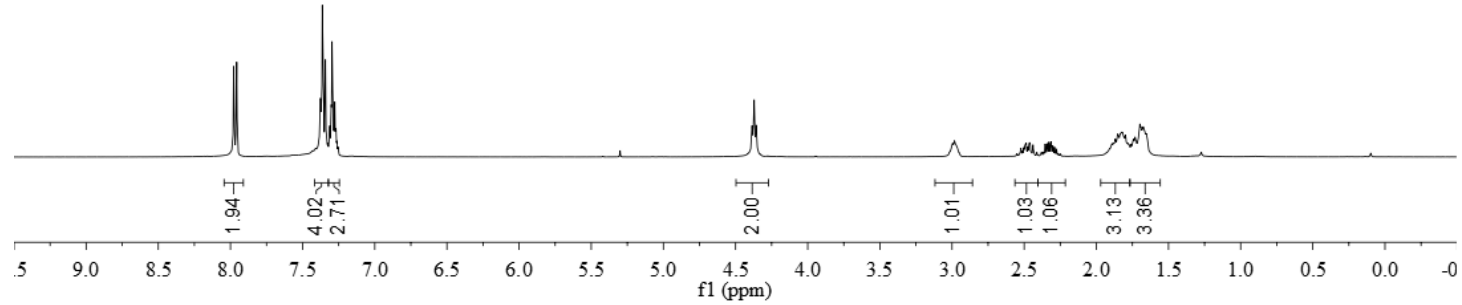<smiles>O=C(OCCCCC(C#CC(F)(F)F)Cc1ccccc1)c1ccc(Cl)cc1</smiles>

$3 \mathrm{i}\left(\mathrm{CDCl}_{3}, 376 \mathrm{MHz}\right)$

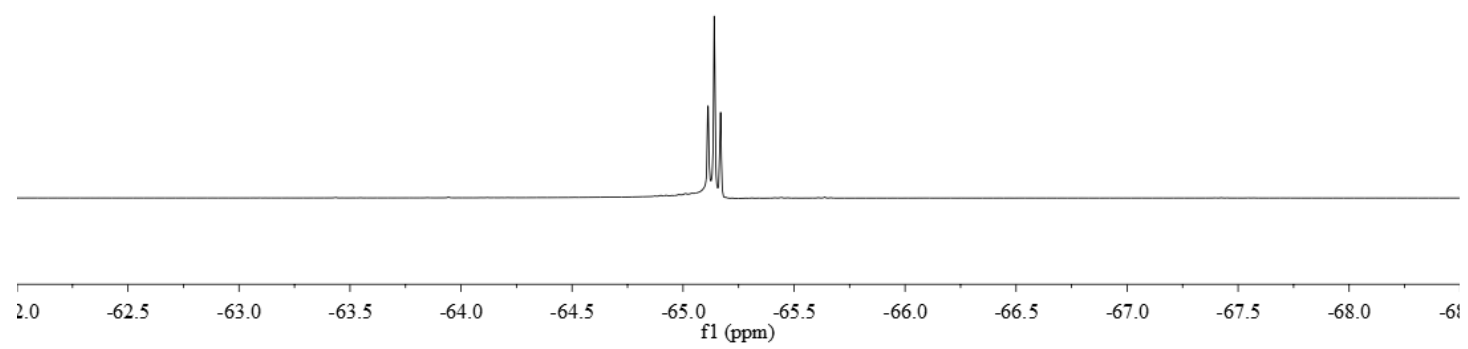



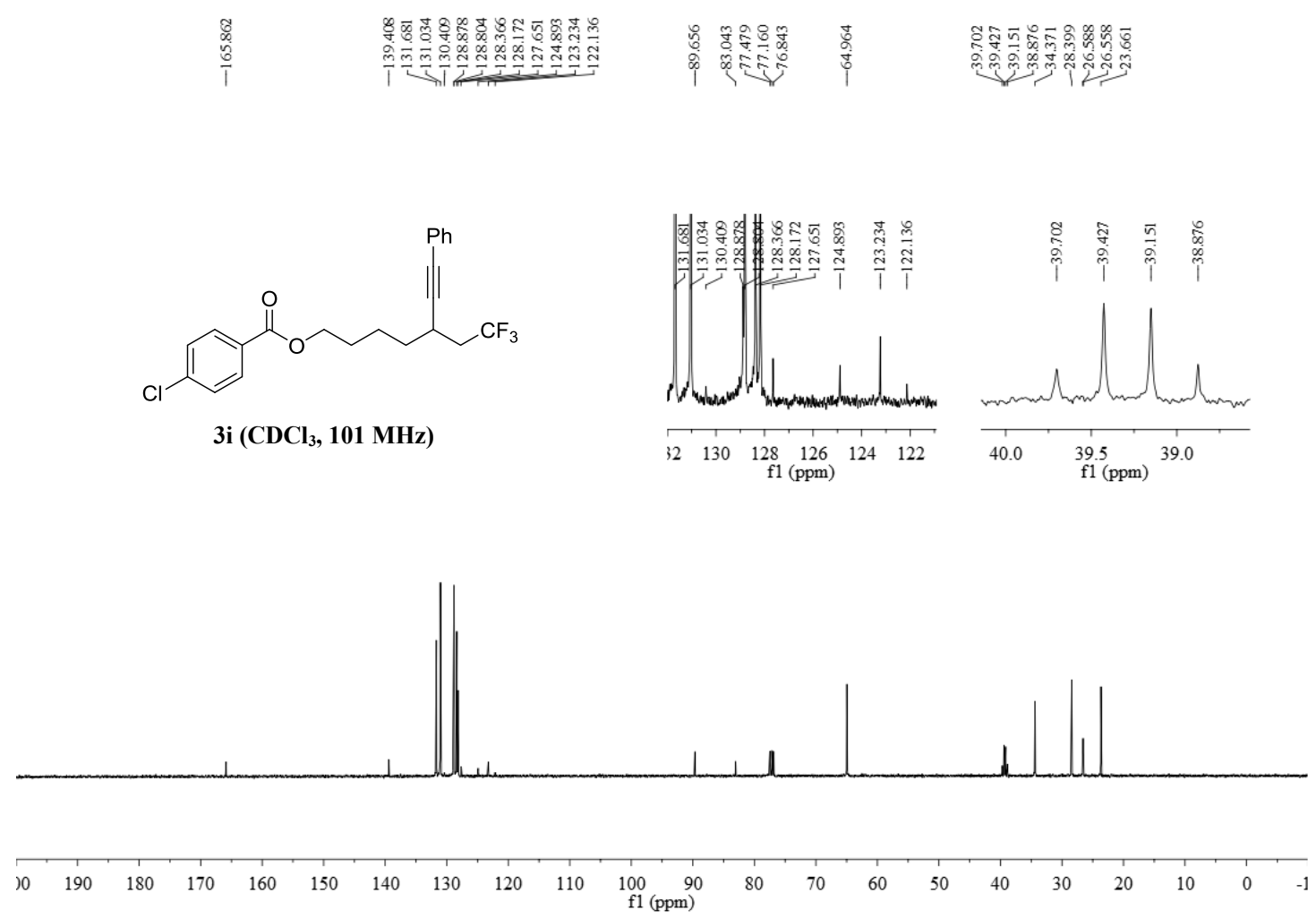

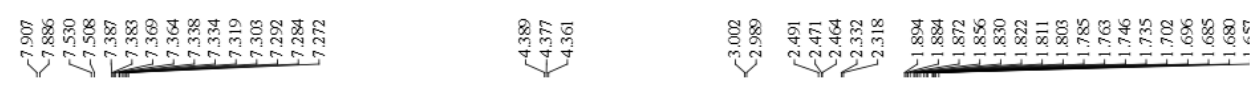

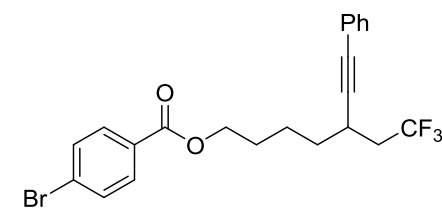

$3 \mathrm{j}\left(\mathrm{CDCl}_{3}, 400 \mathrm{MHz}\right)$

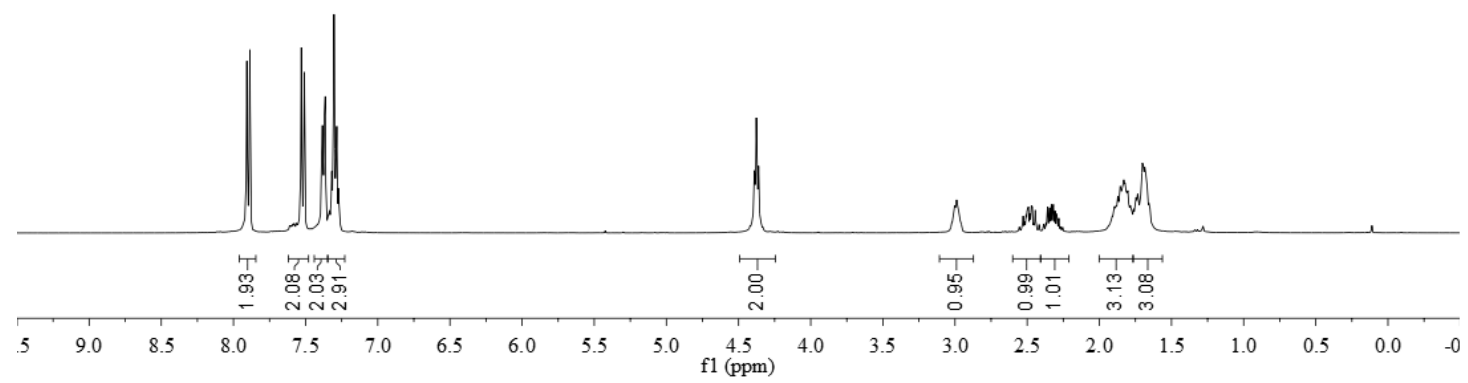




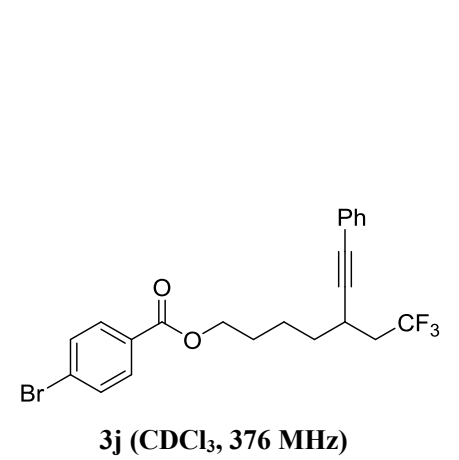

$3 \mathrm{j}\left(\mathrm{CDCl}_{3}, 376 \mathrm{MHz}\right)$

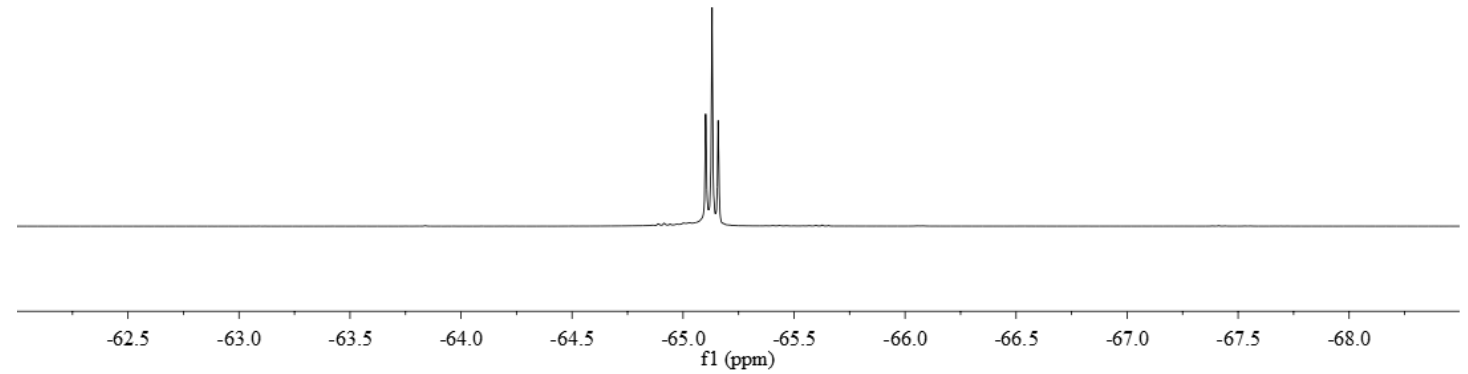

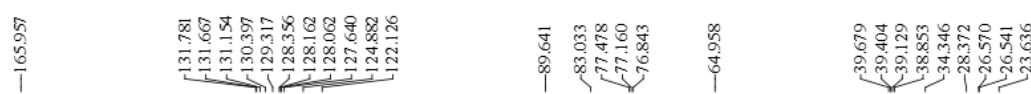
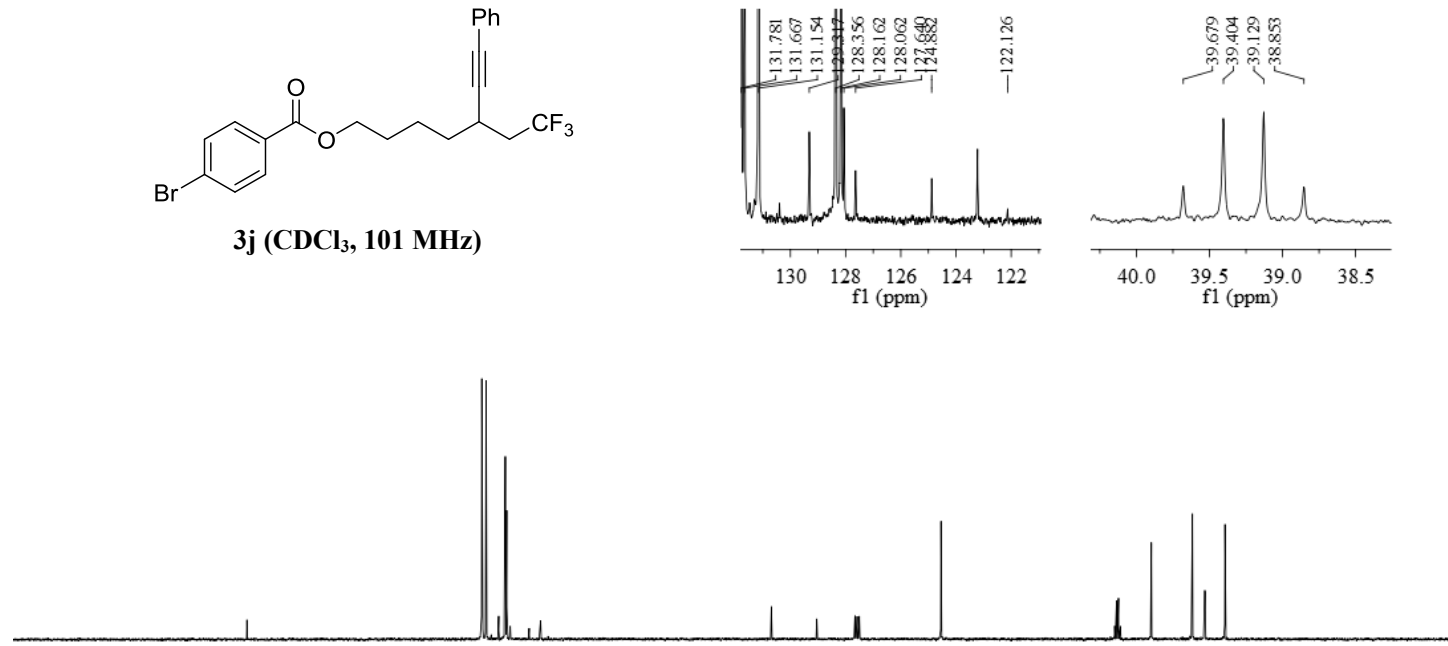

$\begin{array}{lllllllllll}10 & 190 & 180 & 170 & 160 & 150 & 140 & 130 & 120 & 110 & \begin{array}{c}100 \\ \mathrm{f} 1(\mathrm{ppm})\end{array}\end{array}$ 


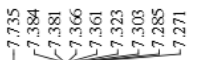

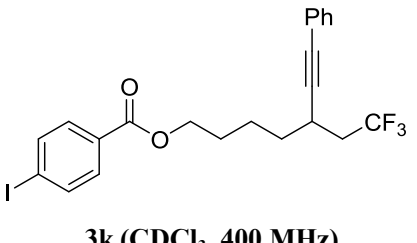

$3 \mathrm{k}\left(\mathrm{CDCl}_{3}, 400 \mathrm{MHz}\right)$
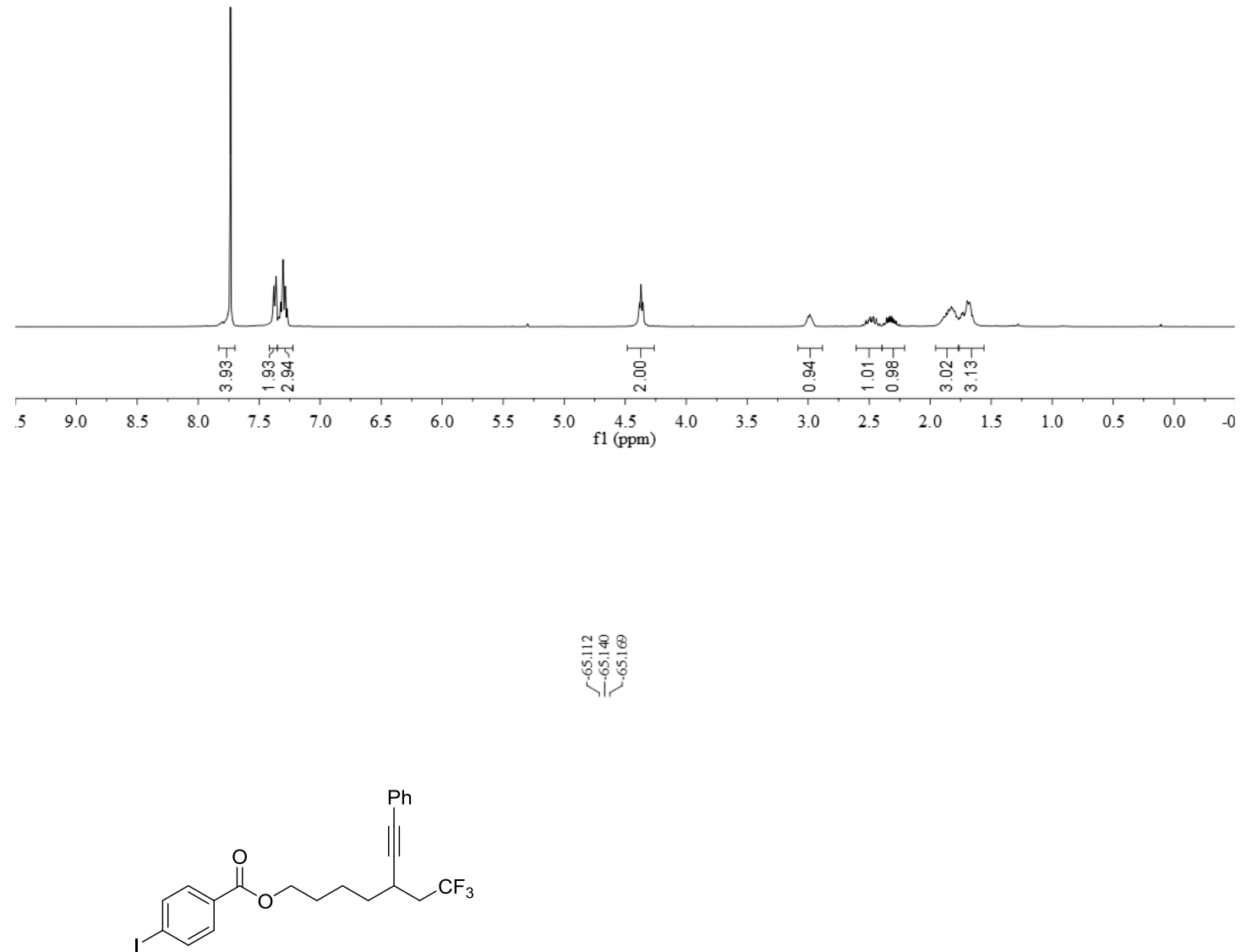

$3 \mathrm{k}\left(\mathrm{CDCl}_{3}, 376 \mathrm{MHz}\right)$

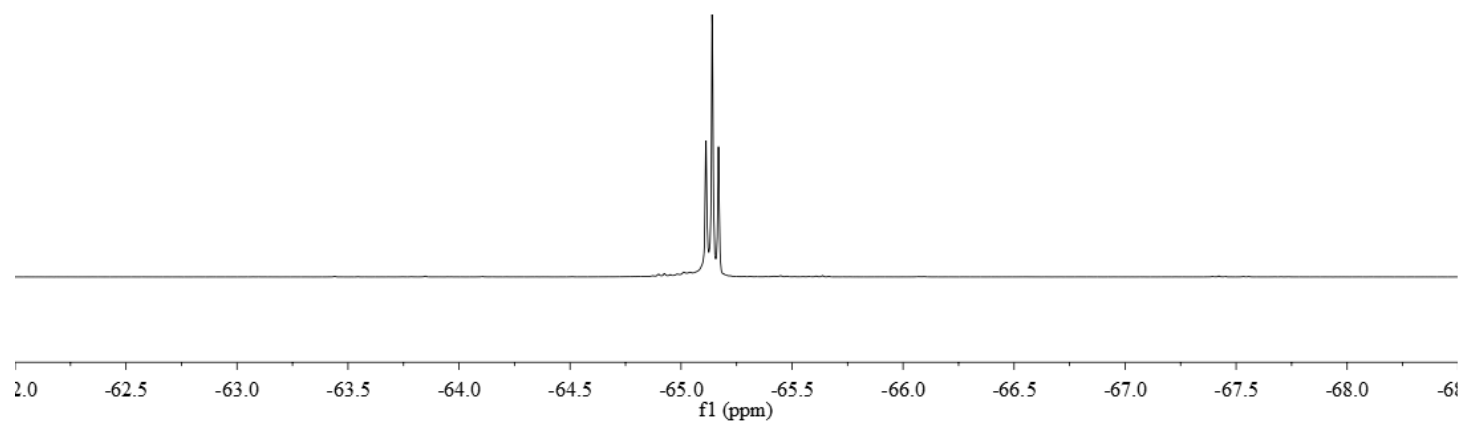




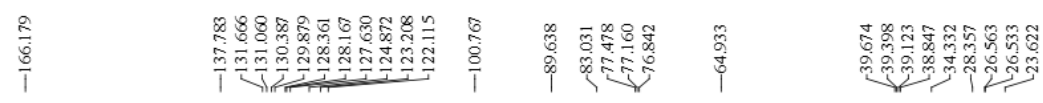
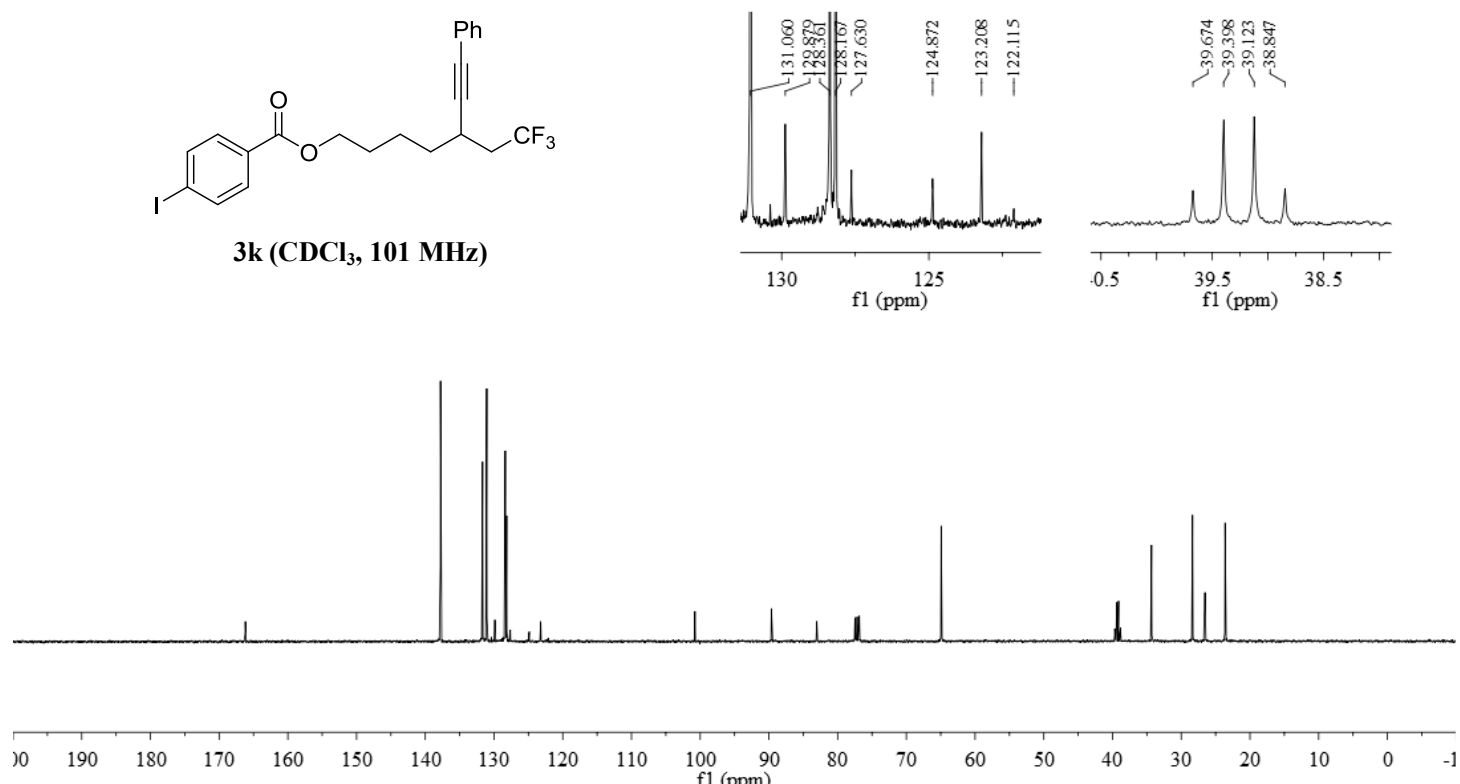

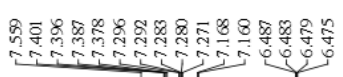

คํำ ले

ఇू.

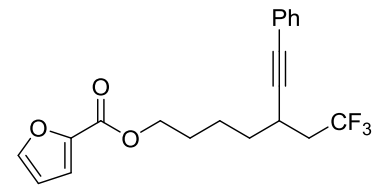

$3 \mathrm{l}\left(\mathrm{CDCl}_{3}, 400 \mathrm{MHz}\right)$

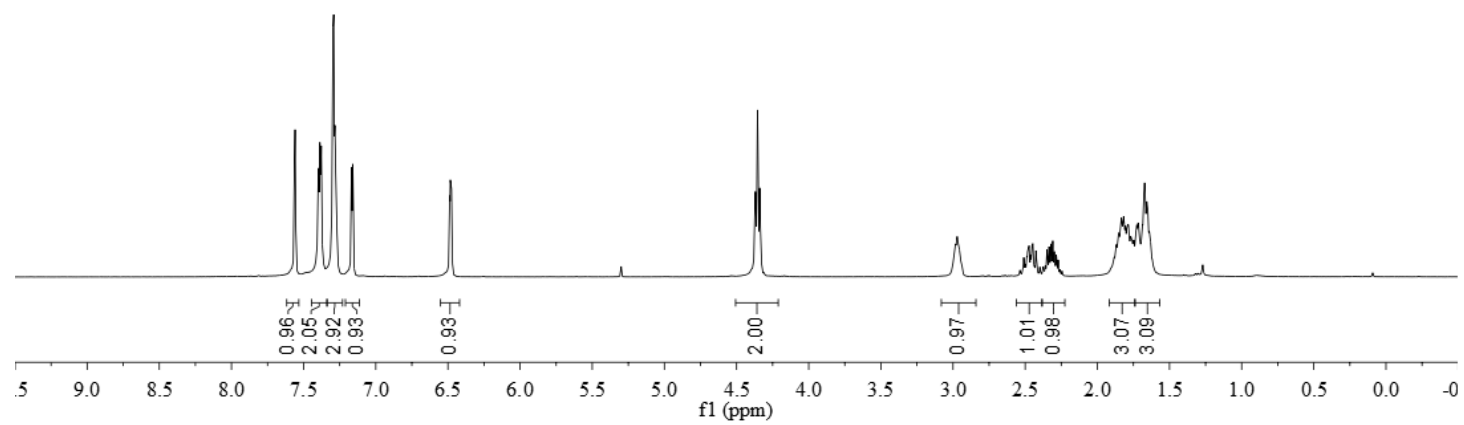


308
70
0

$\underbrace{2}$

$3 \mathrm{l}\left(\mathrm{CDCl}_{3}, 376 \mathrm{MHz}\right)$
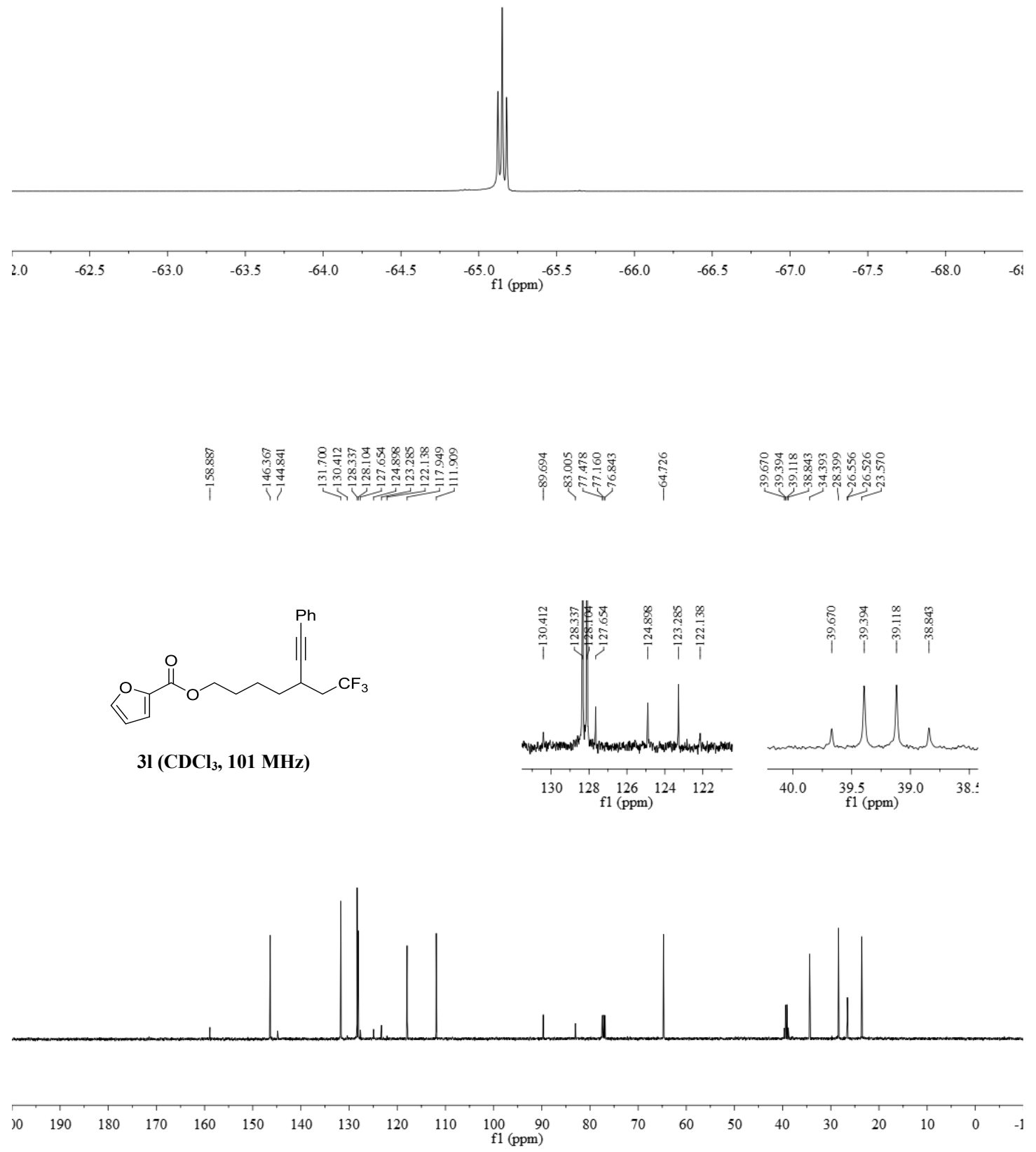

S53 


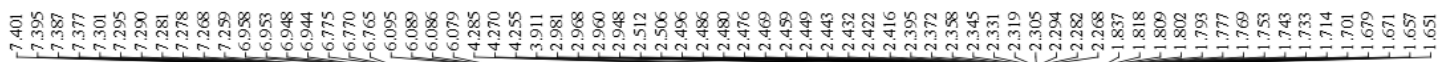
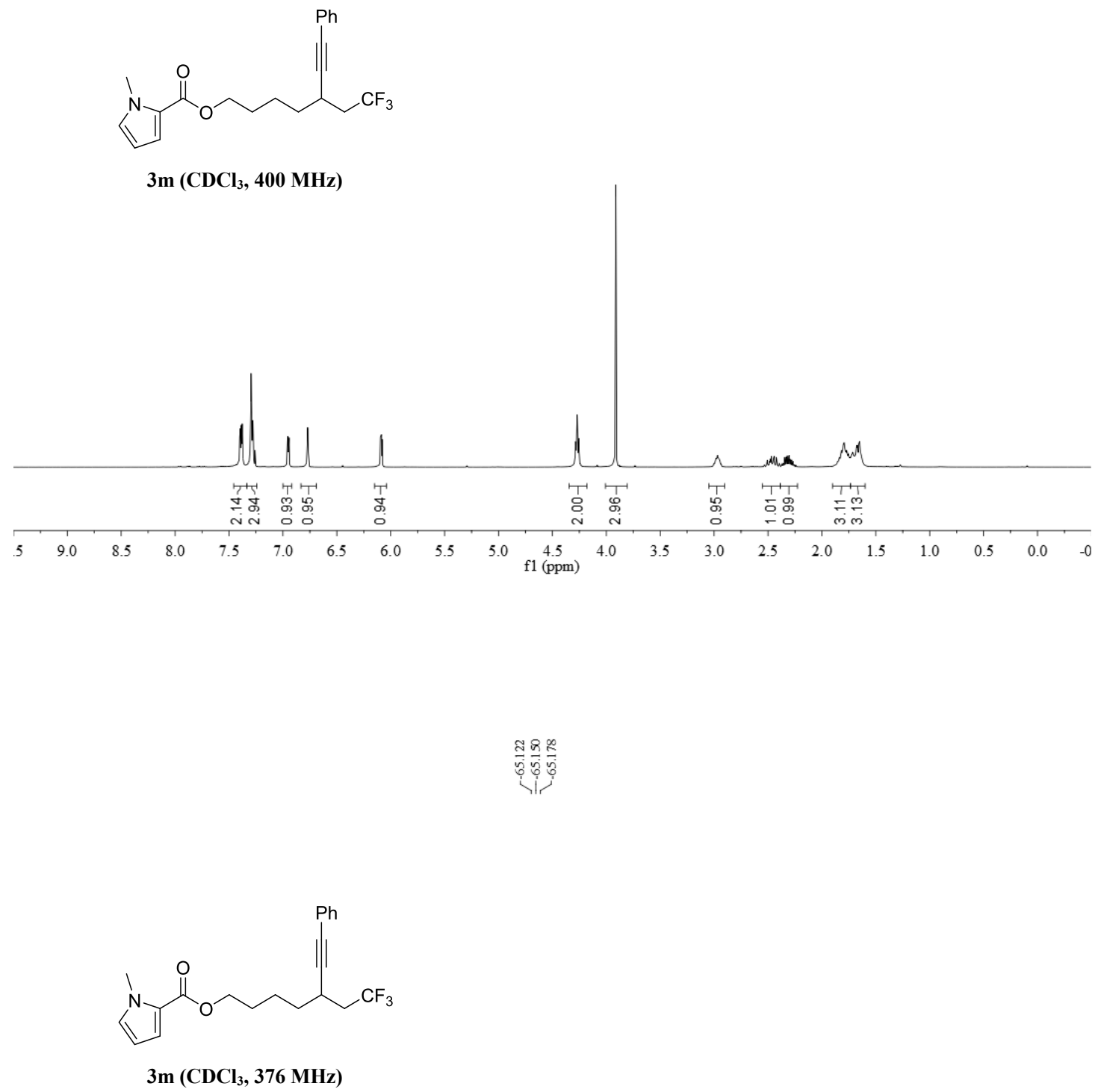

ํํำ
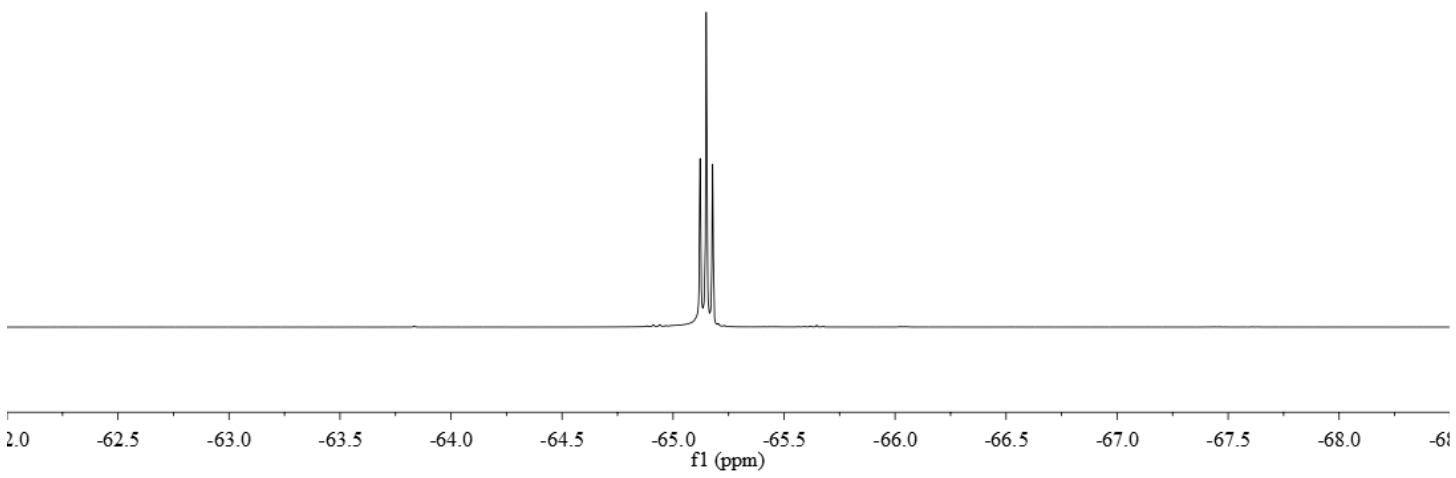

S54 
<smiles>Cn1cccc1C(=O)OCCCCC(C#CC(F)(F)F)CC(F)(F)F</smiles>

$3 \mathrm{~m}\left(\mathrm{CDCl}_{3}, 101 \mathrm{MHz}\right)$
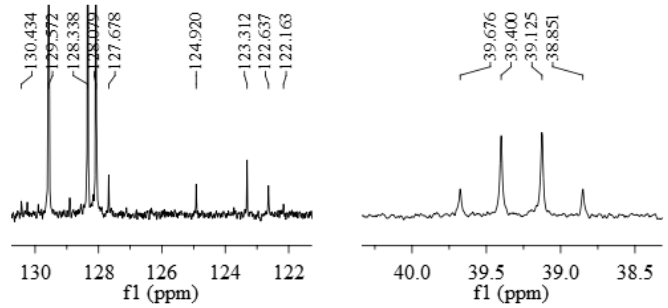

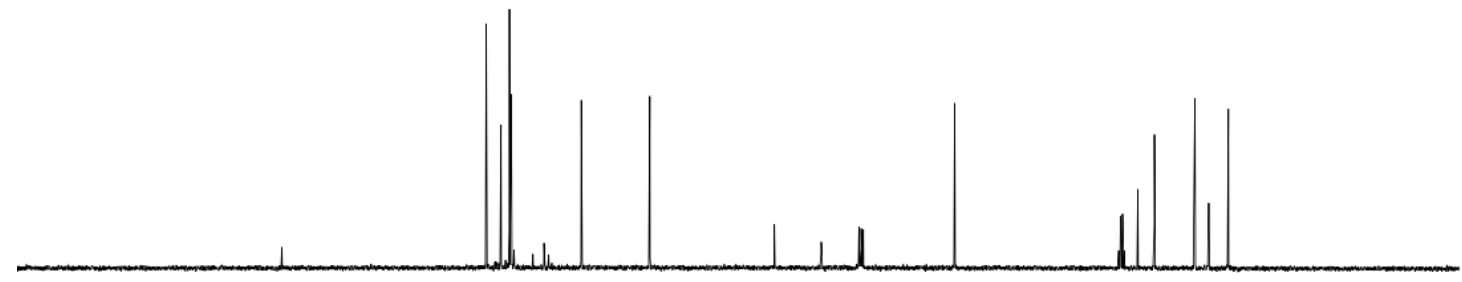

$\begin{array}{lllllllllllllllllllllll}0 & 190 & 180 & 170 & 160 & 150 & 140 & 130 & 120 & 110 & 100 & 90 & 80 & 70 & 60 & 50 & 40 & 30 & 20 & 10 & 0 & -1\end{array}$

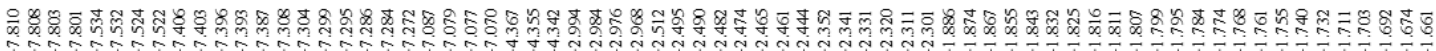<smiles>O=C(OCCCCC(C#CPc1ccccc1)CC(F)(F)F)c1cccs1</smiles>

3n $\left(\mathrm{CDCl}_{3}, \mathbf{5 0 0} \mathrm{MHz}\right)$

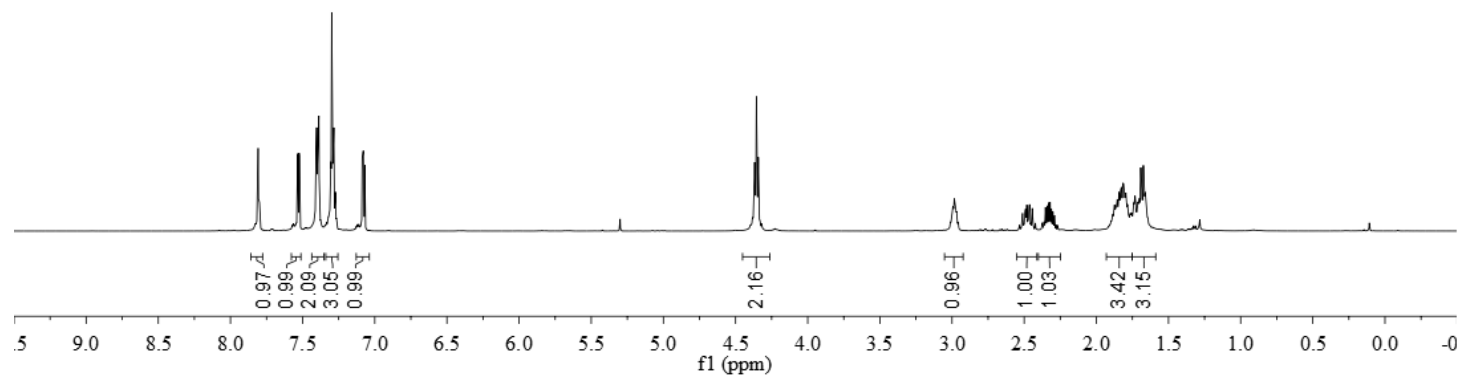




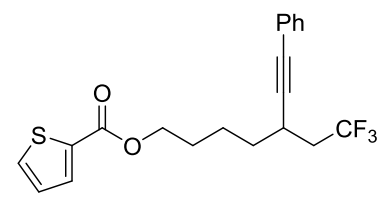

3n $\left(\mathrm{CDCl}_{3}, 470 \mathrm{MHz}\right)$

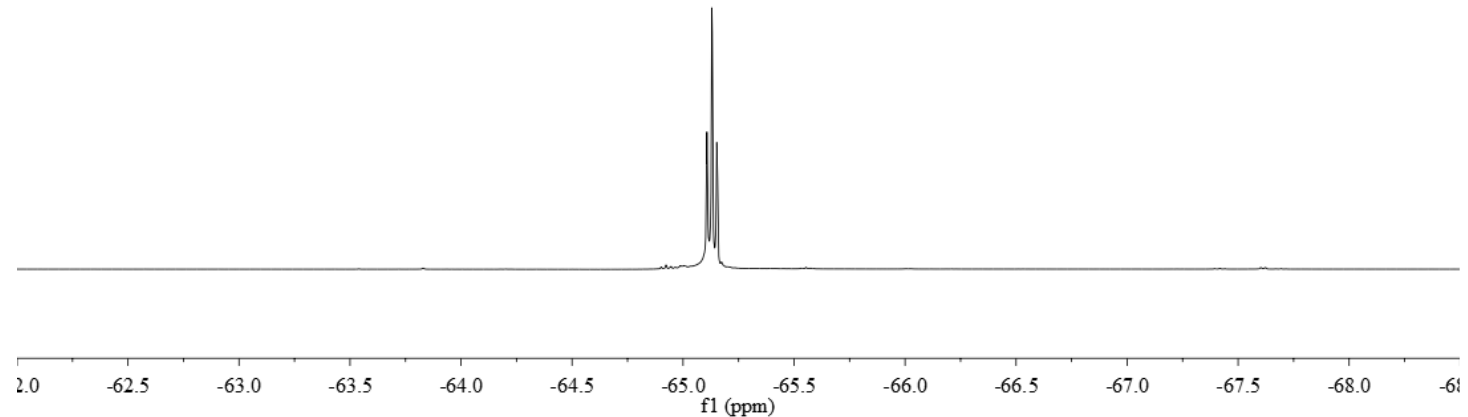

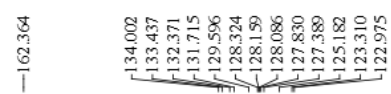

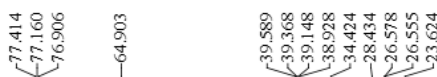

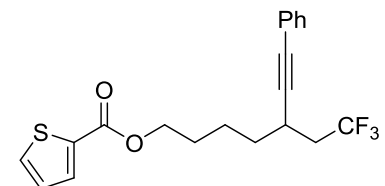

3n $\left(\mathrm{CDCl}_{3}, 126 \mathrm{MHz}\right)$
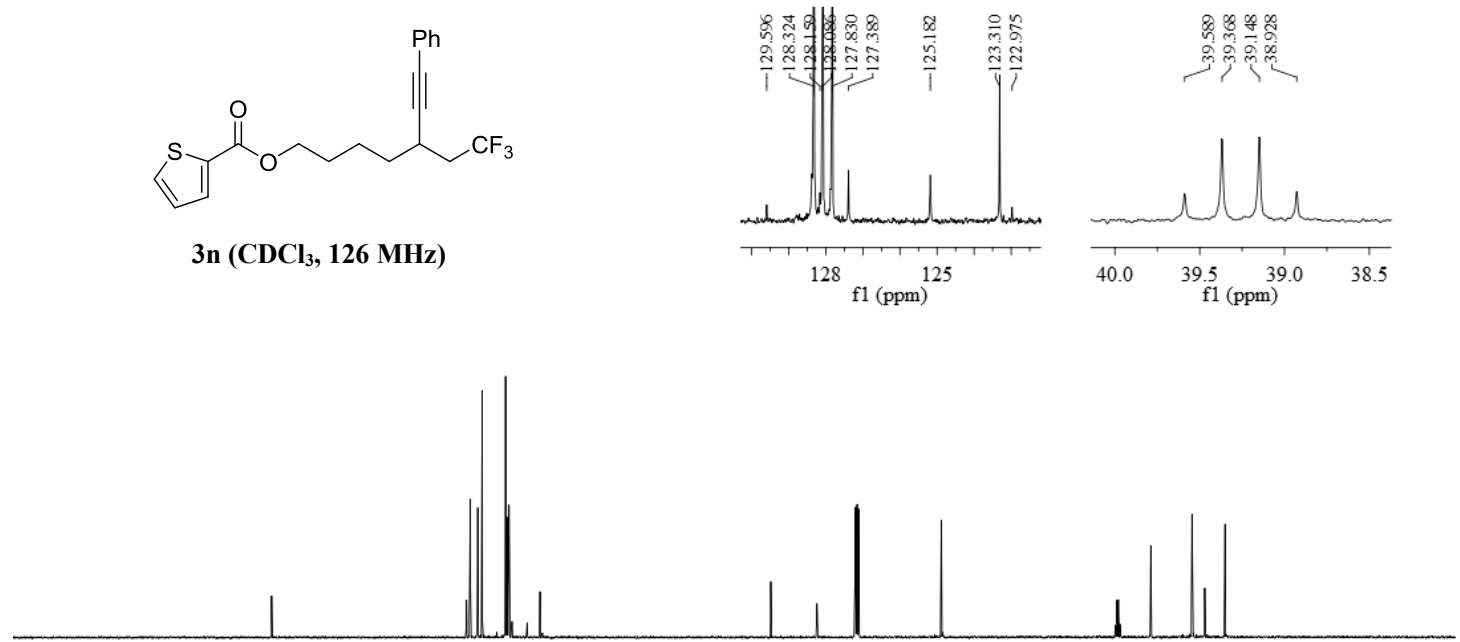

$\begin{array}{lllllllllll}0 & 190 & 180 & 170 & 160 & 150 & 140 & 130 & 120 & 110 & 100 \quad 90 \\ \mathrm{f} 1(\mathrm{ppm})\end{array}$ 
<smiles>O=C(OCCCCC(C=Cc1ccccc1)C(F)(F)F)c1ccccn1</smiles>

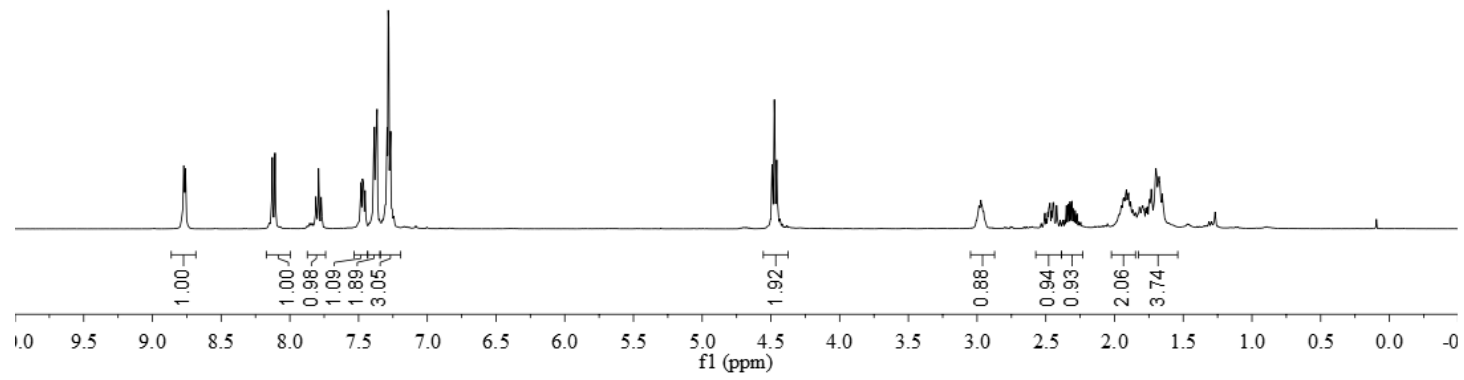<smiles>C=C=CC(CCCCOC(=O)c1ccccn1)CC(F)(F)F</smiles>

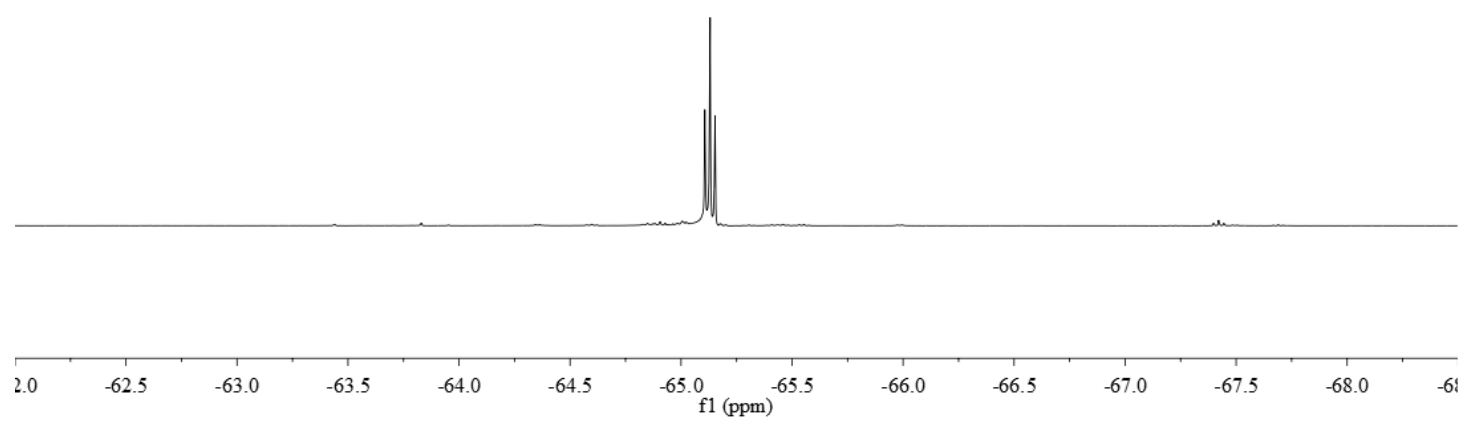



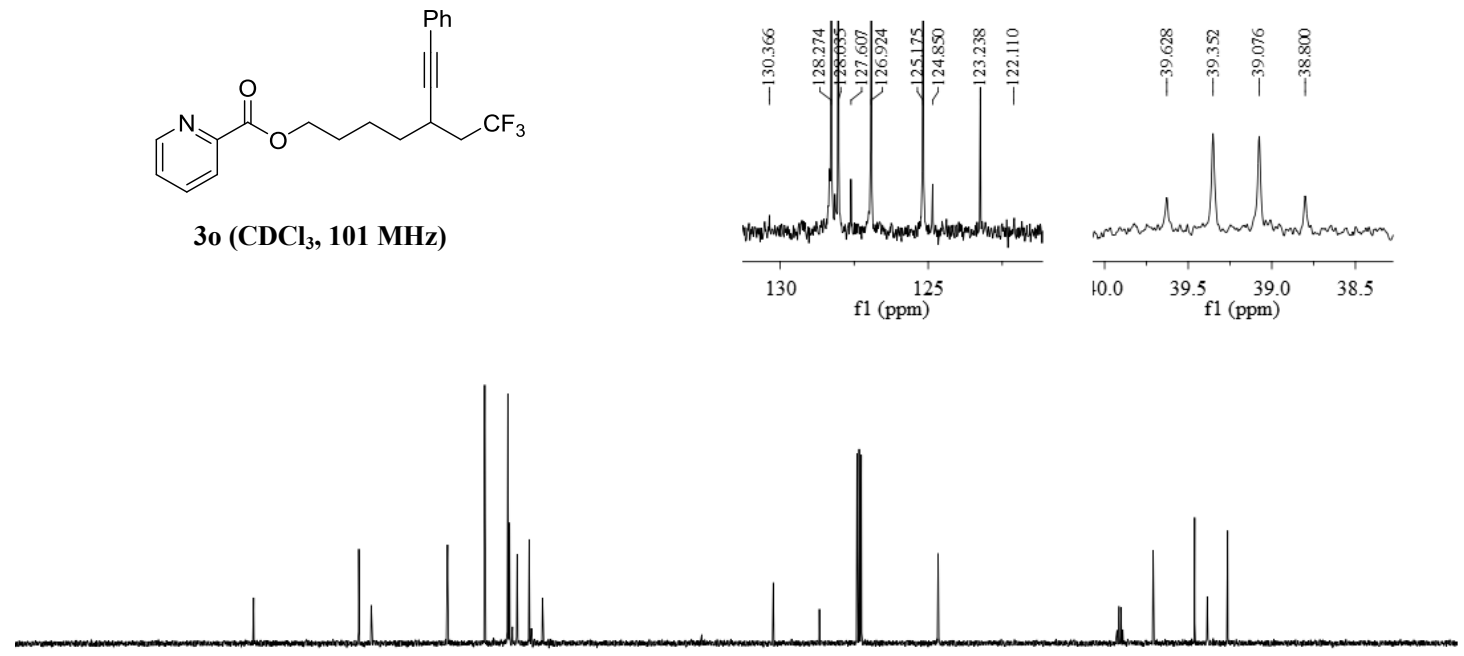

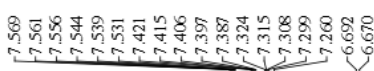

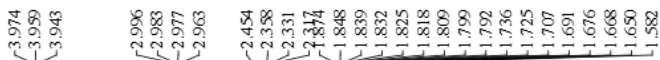<smiles>FC(F)(F)CC(C#Cc1ccccc1)CCCCOc1ccc(I)cc1</smiles>

3p $\left(\mathrm{CDCl}_{3}, 400 \mathrm{MHz}\right)$

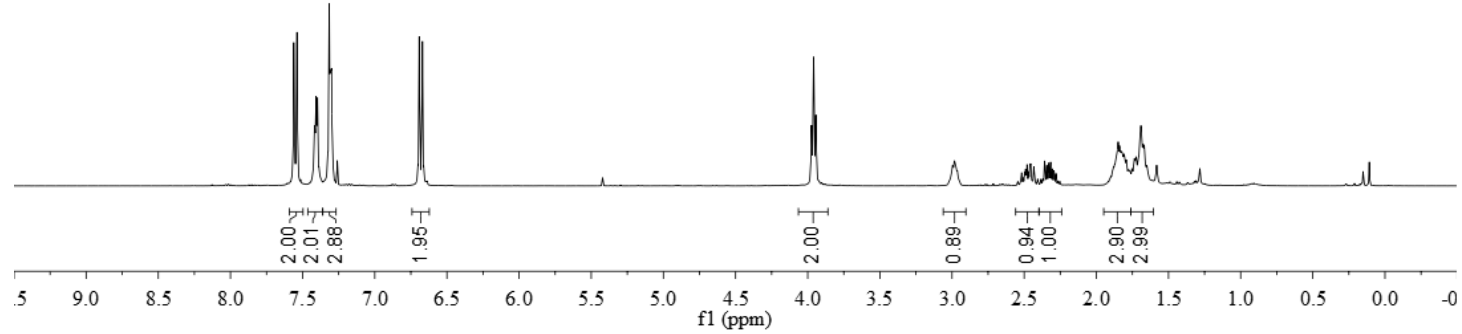




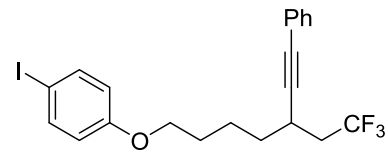

$3 p\left(\mathrm{CDCl}_{3}, 470 \mathrm{MHz}\right)$

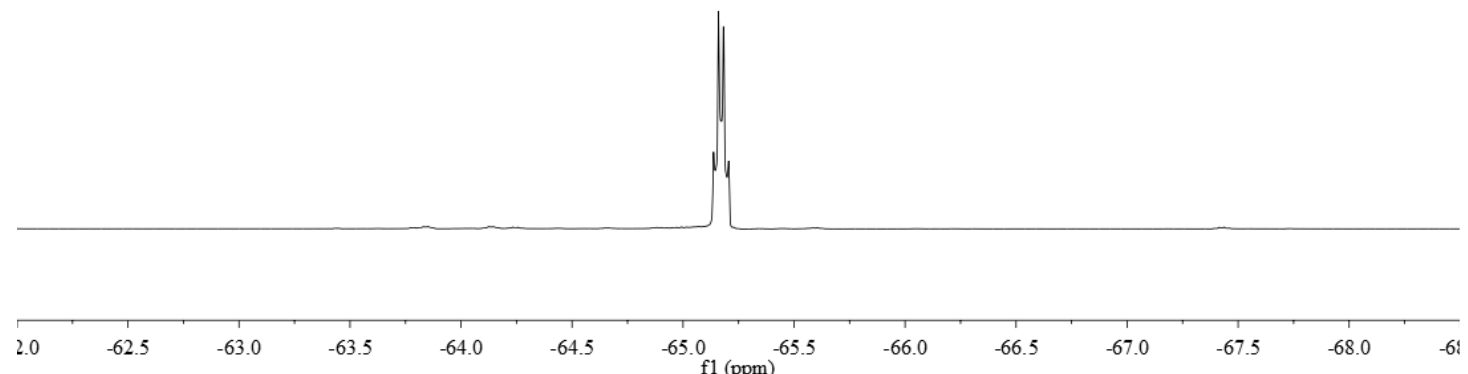

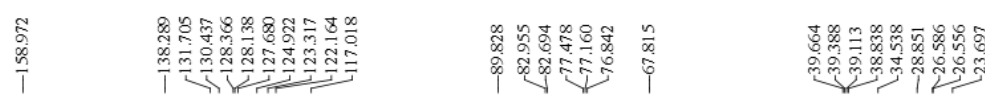

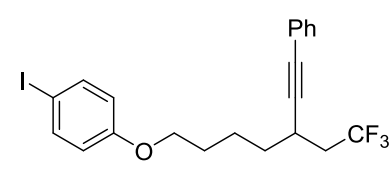

3p $\left(\mathrm{CDCl}_{3}, 101 \mathrm{MHz}\right)$
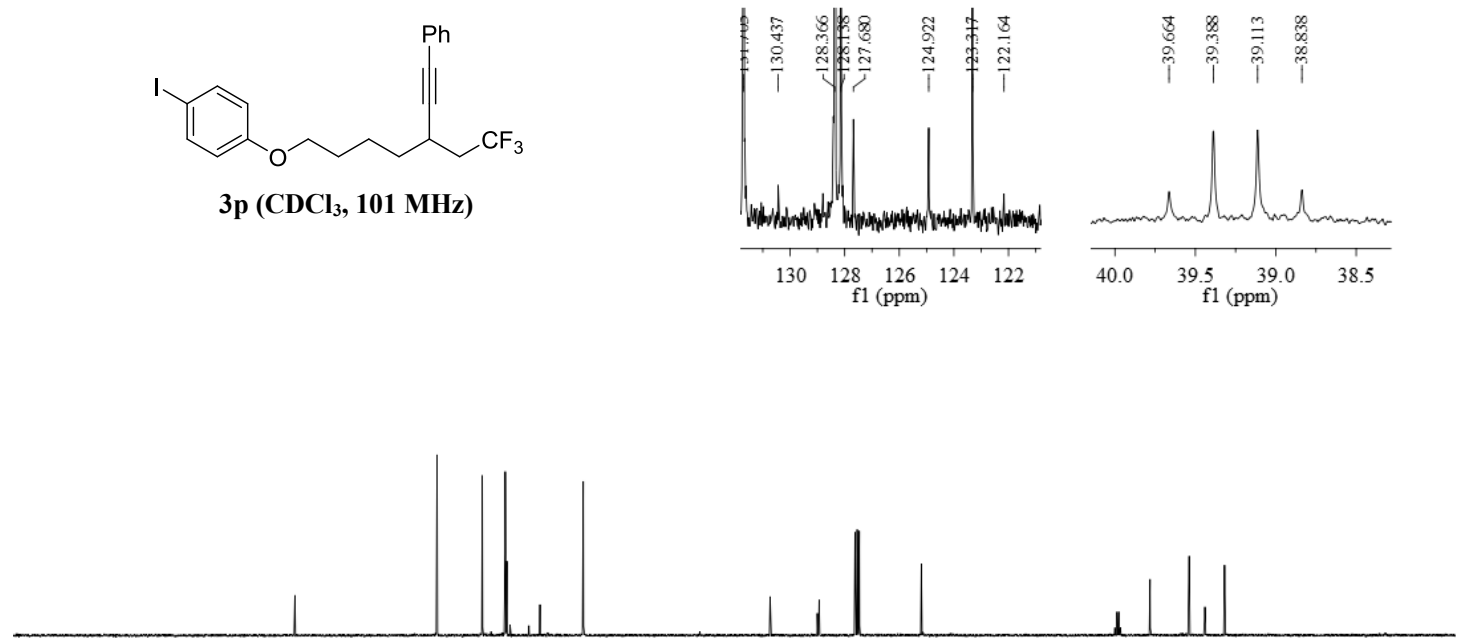

$\begin{array}{lllllllllll}10 & 190 & 180 & 170 & 160 & 150 & 140 & 130 & 120 & 110 & \begin{array}{c}100 \\ \mathrm{f} 1(\mathrm{ppm})\end{array}\end{array}$ 


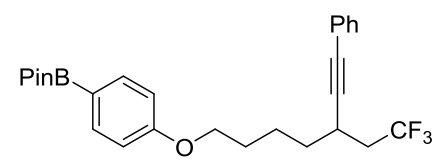

$3 q\left(\mathrm{CDCl}_{3}, 500 \mathrm{MHz}\right)$

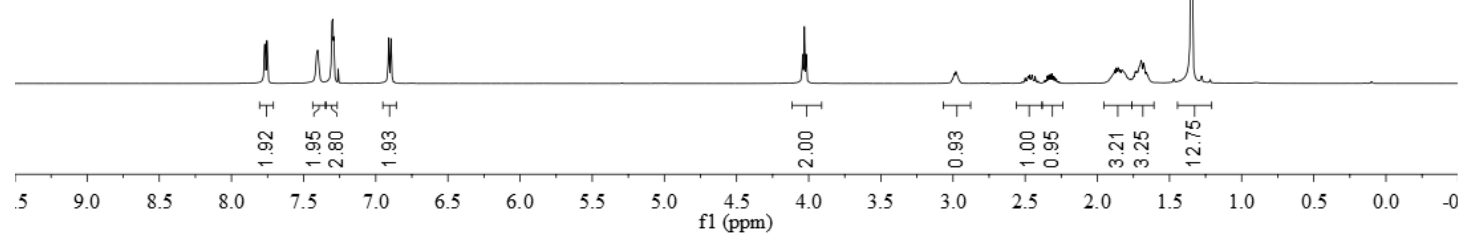

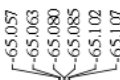

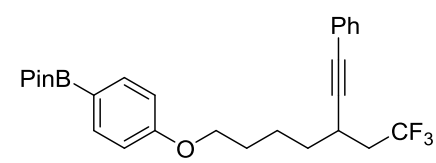

$3 q\left(\mathrm{CDCl}_{3}, 470 \mathrm{MHz}\right)$

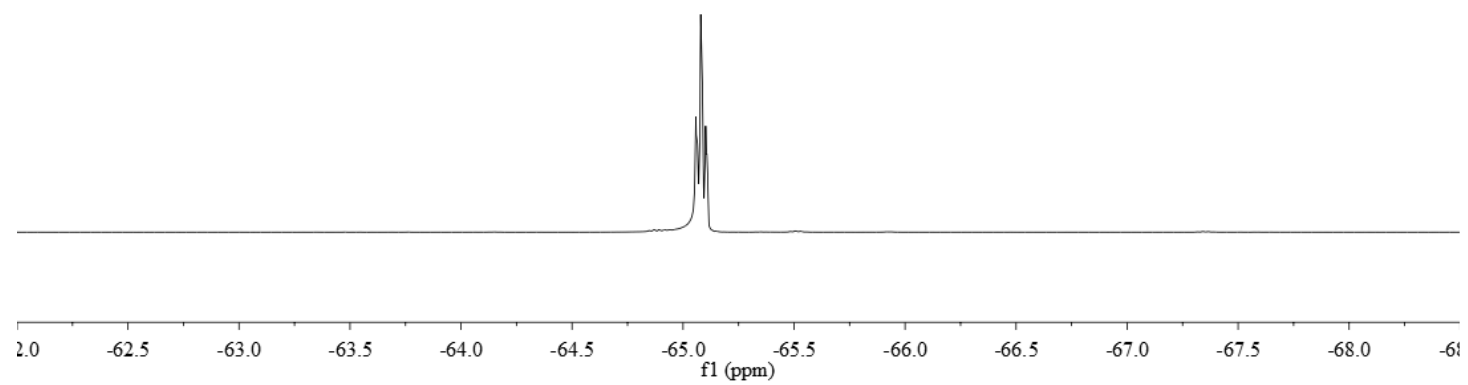



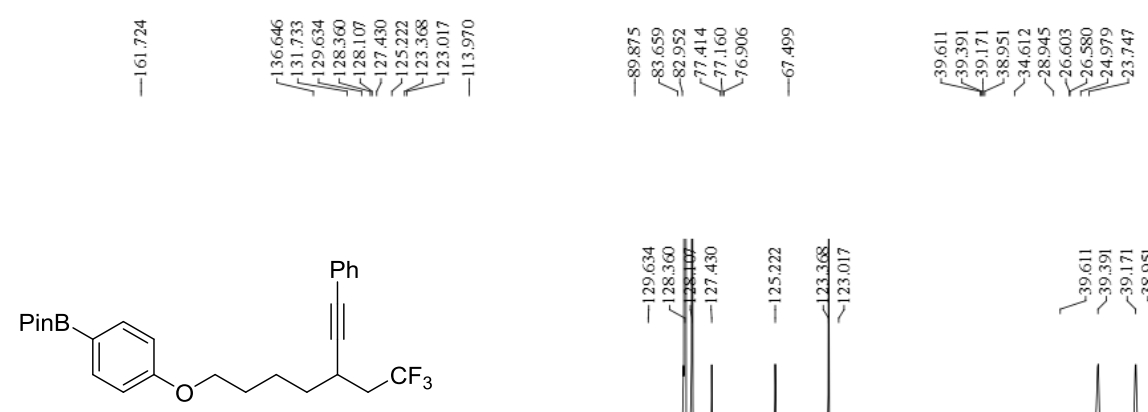

$3 q\left(\mathrm{CDCl}_{3}, 126 \mathrm{MHz}\right)$
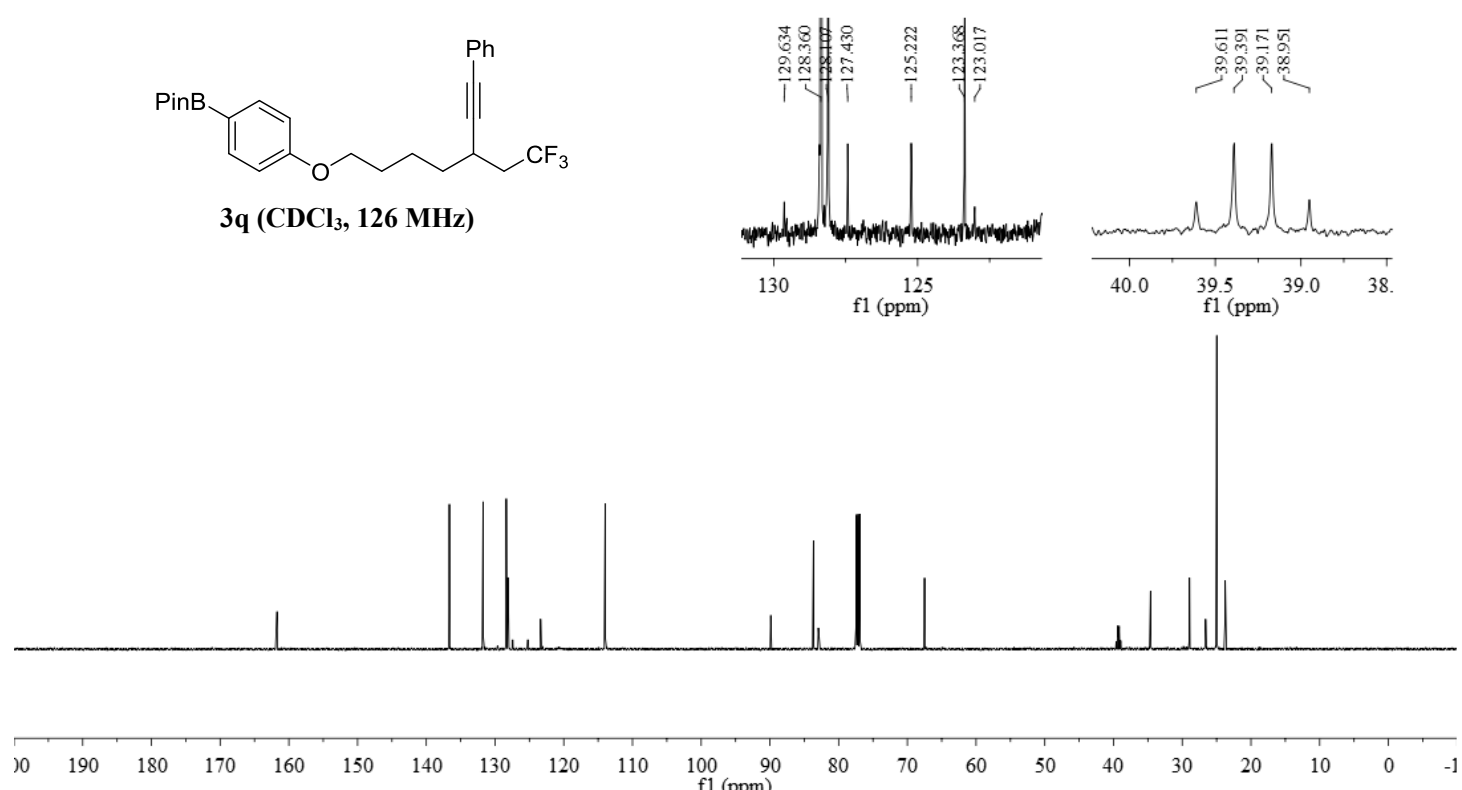

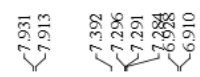

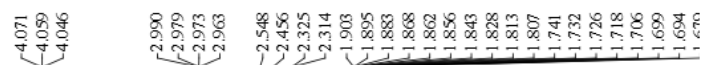<smiles>CC(=O)c1ccc(OCCCCC(C#Cc2ccccc2)CC(F)(F)F)cc1</smiles>

$3 \mathrm{r}\left(\mathrm{CDCl}_{3}, 500 \mathrm{MHz}\right)$

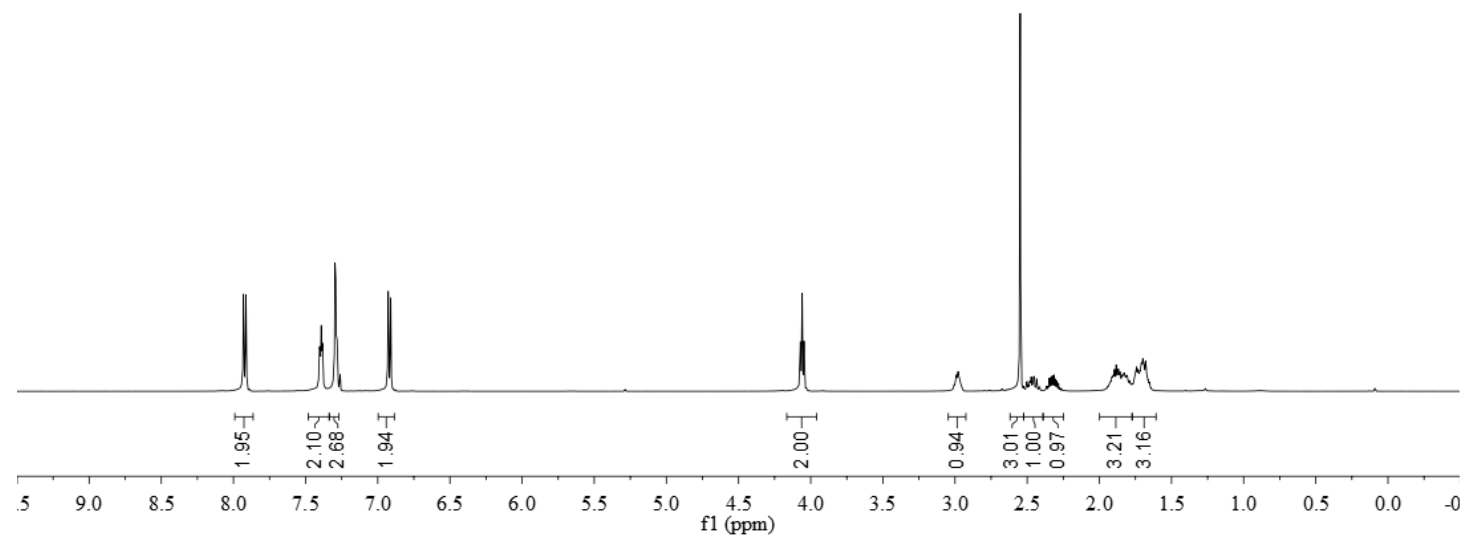




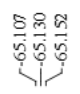
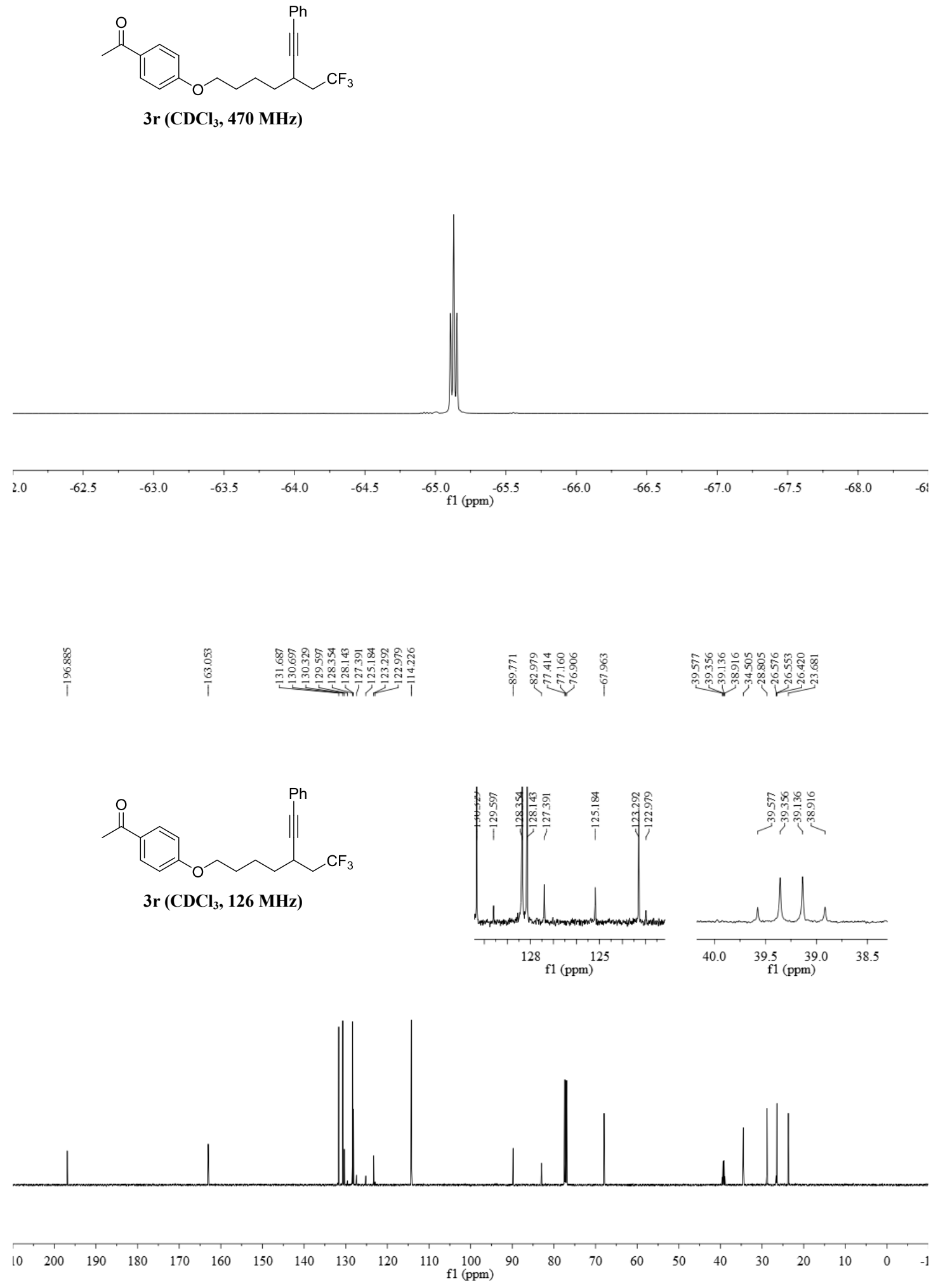

S62 


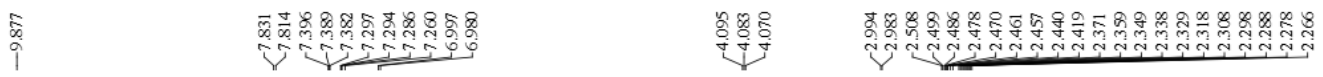<smiles>O=Cc1ccc(OCCCCC(C#Cc2ccccc2)CC(F)(F)F)cc1</smiles>

3s $\left(\mathrm{CDCl}_{3}, 500 \mathrm{MHz}\right)$

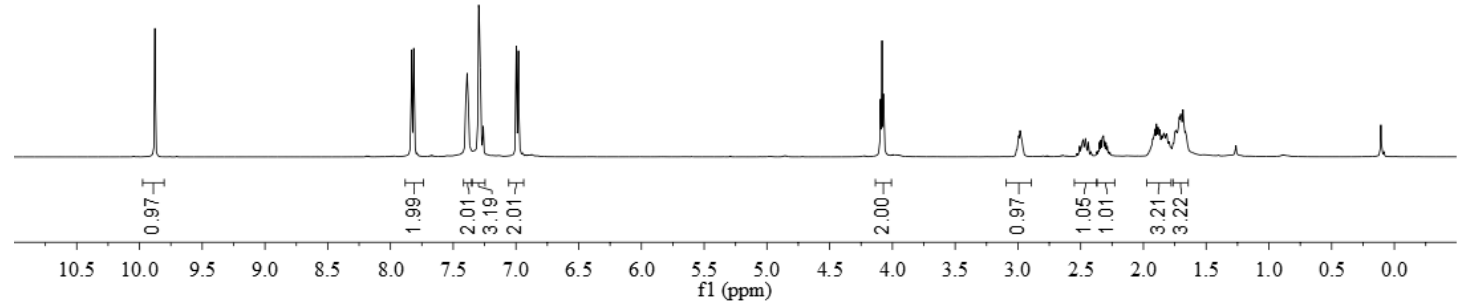<smiles>O=Cc1ccc(OCCCCC(C#CCC(F)(F)c2ccccc2)CC(F)(F)F)cc1</smiles>

3s ( $\left.\mathrm{CDCl}_{3}, 470 \mathrm{MHz}\right)$

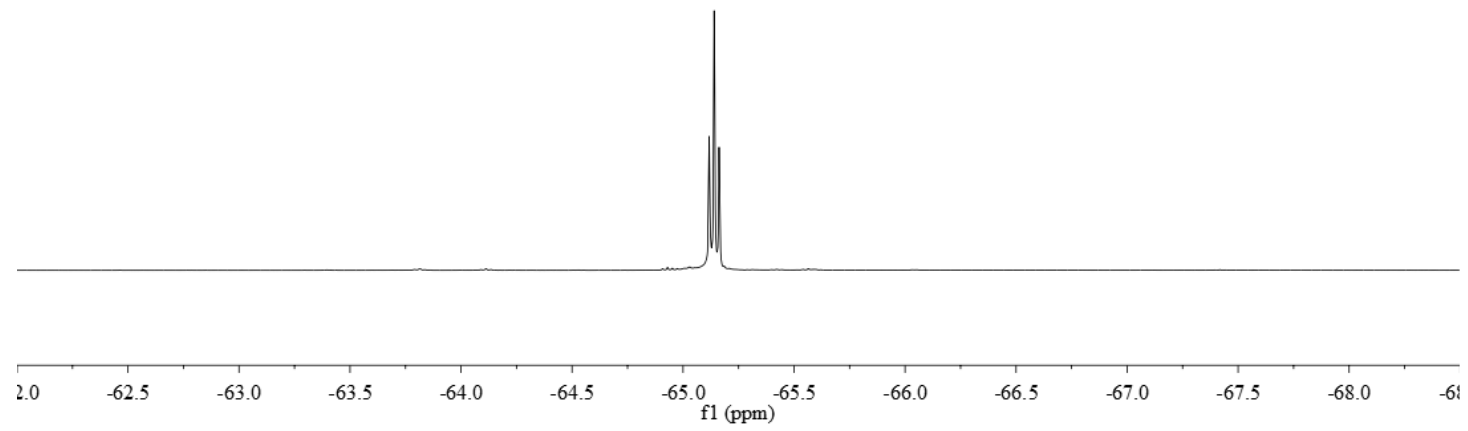



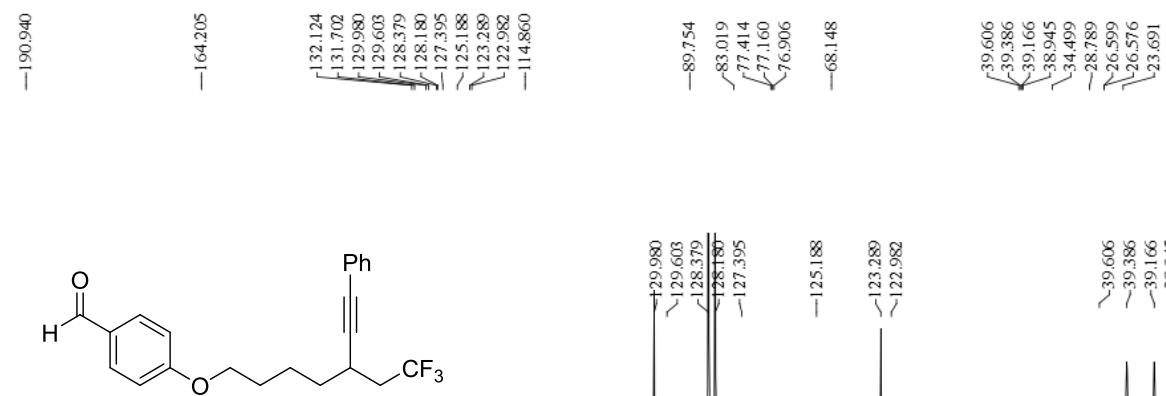

3s $\left(\mathrm{CDCl}_{3}, 126 \mathrm{MHz}\right)$
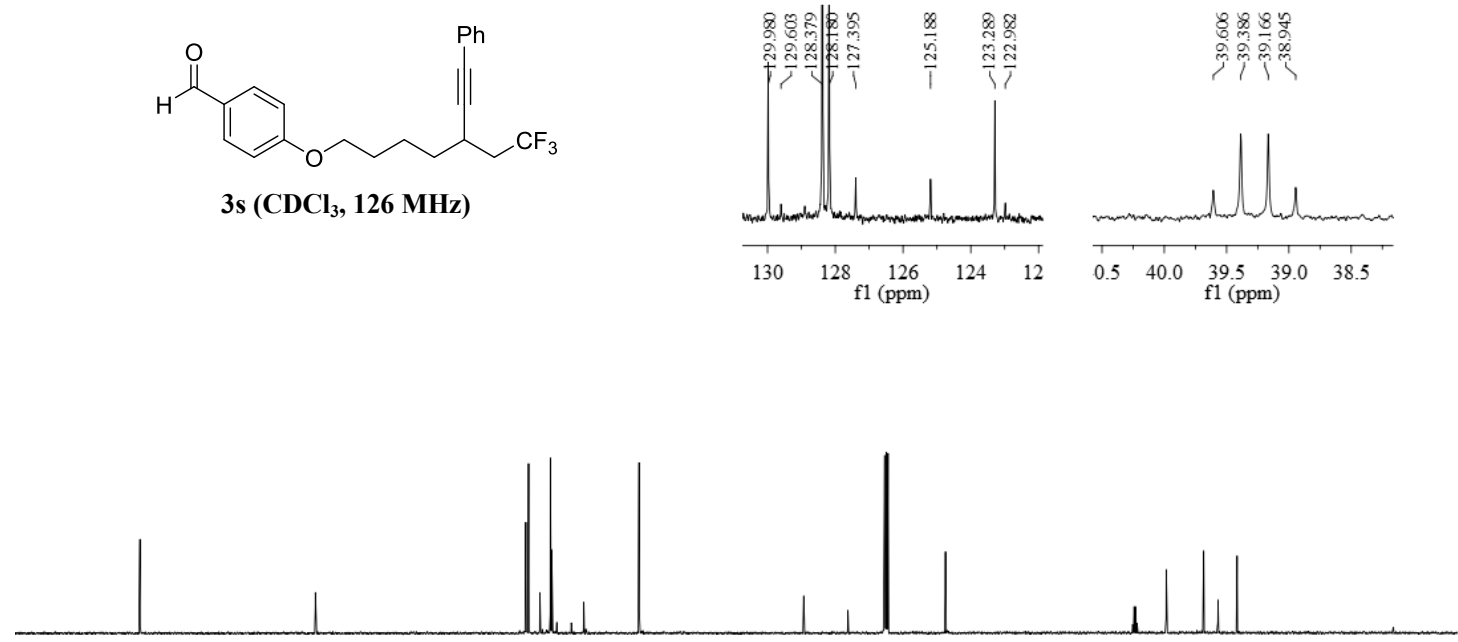

$\begin{array}{llllllllllllllllllllllllllllllll}10 & 200 & 190 & 180 & 170 & 160 & 150 & 140 & 130 & 120 & 110 & 100 & 90 & 80 & 70 & 60 & 50 & 40 & 30 & 20 & 10 & 0 & -1\end{array}$

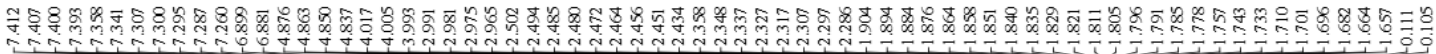<smiles>CS(=O)(=O)OC(c1ccc(OCCCCC(C#CPc2ccccc2)CC(F)(F)F)cc1)C(F)(F)F</smiles>

$3 s^{\prime}\left(\mathrm{CDCl}_{3}, 500 \mathrm{MHz}\right)$

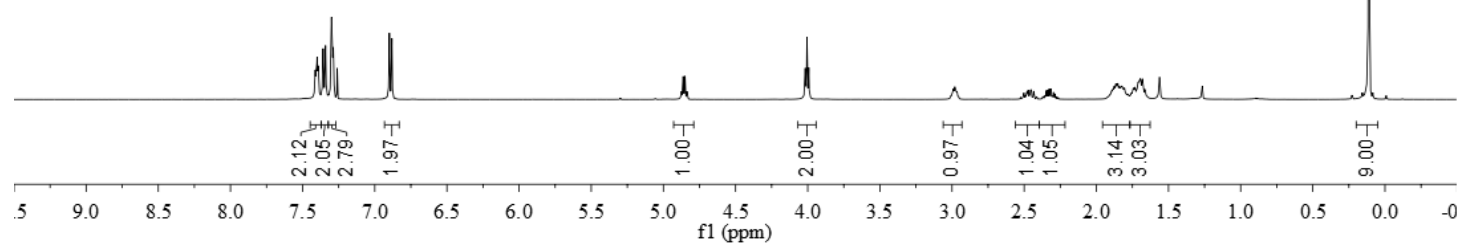




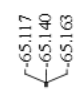<smiles>COC(c1ccccc1)c1ccc(OCCCCC(C#CC(F)(F)F)CC(F)(F)F)cc1</smiles>

$3 s^{\prime}\left(\mathrm{CDCl}_{3}, 470 \mathrm{MHz}\right)$

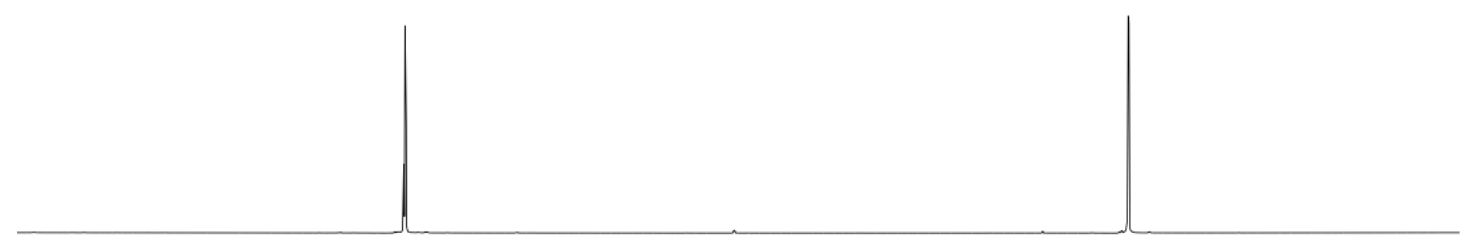

\begin{tabular}{|c|c|c|c|c|c|c|c|c|c|c|c|c|}
\hline-59 & -61 & -63 & -65 & -67 & -69 & ${ }^{-71} 1(\mathrm{ppm}){ }^{-73}$ & -75 & -77 & -79 & -81 & -83 & -85 \\
\hline
\end{tabular}

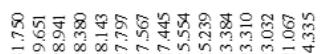

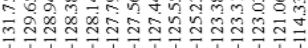

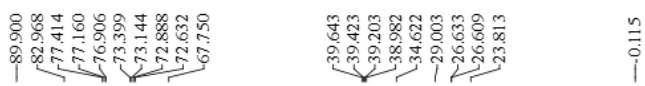<smiles>OC(c1ccccc1)c1ccc(OCCCCC(C#CC(F)(F)F)CC(F)(F)F)cc1</smiles>

3s' $\left(\mathrm{CDCl}_{3}, 126 \mathrm{MHz}\right)$
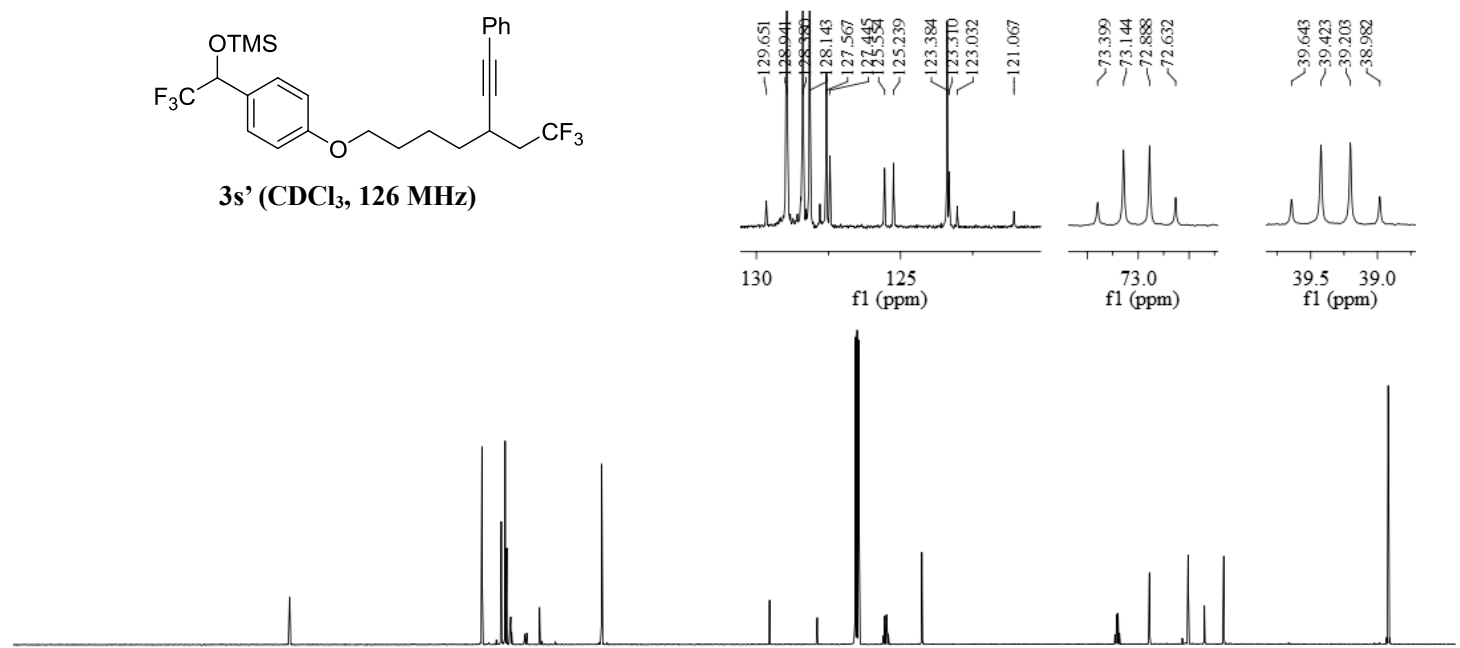

$\begin{array}{lllllllllllllllllllll}10 & 190 & 180 & 170 & 160 & 150 & 140 & 130 & 120 & 110 & \underset{\mathrm{f} 1(\mathrm{ppm})}{100} & 80 & 70 & 60 & 50 & 40 & 30 & 20 & 10 & 0 & -1\end{array}$ 


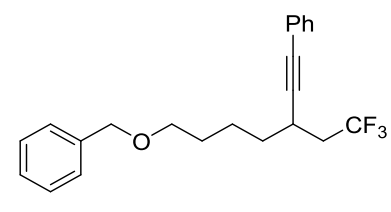

$3 t\left(\mathrm{CDCl}_{3}, 400 \mathrm{MHz}\right)$
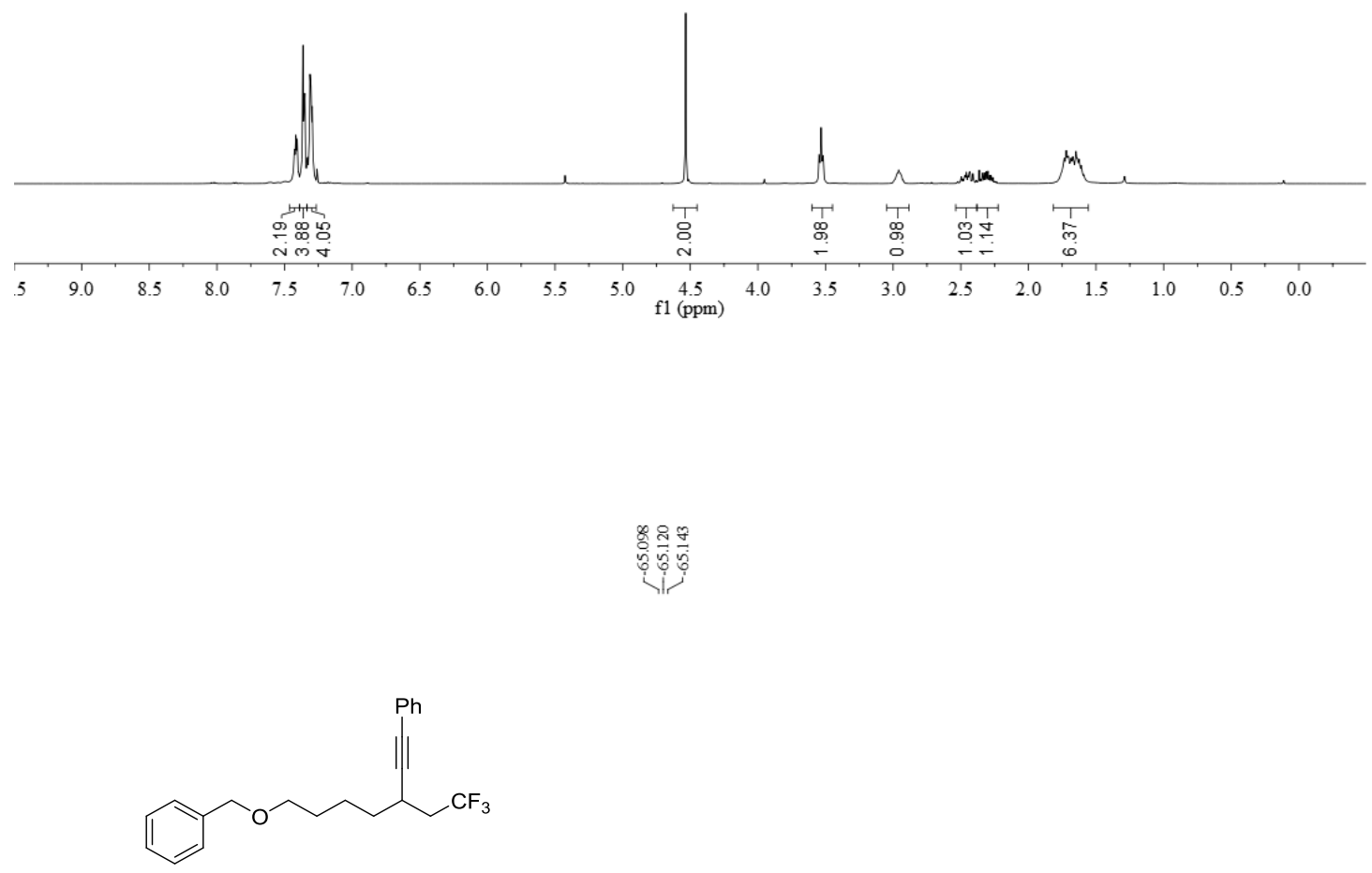

$3 t\left(\mathrm{CDCl}_{3}, 470 \mathrm{MHz}\right)$

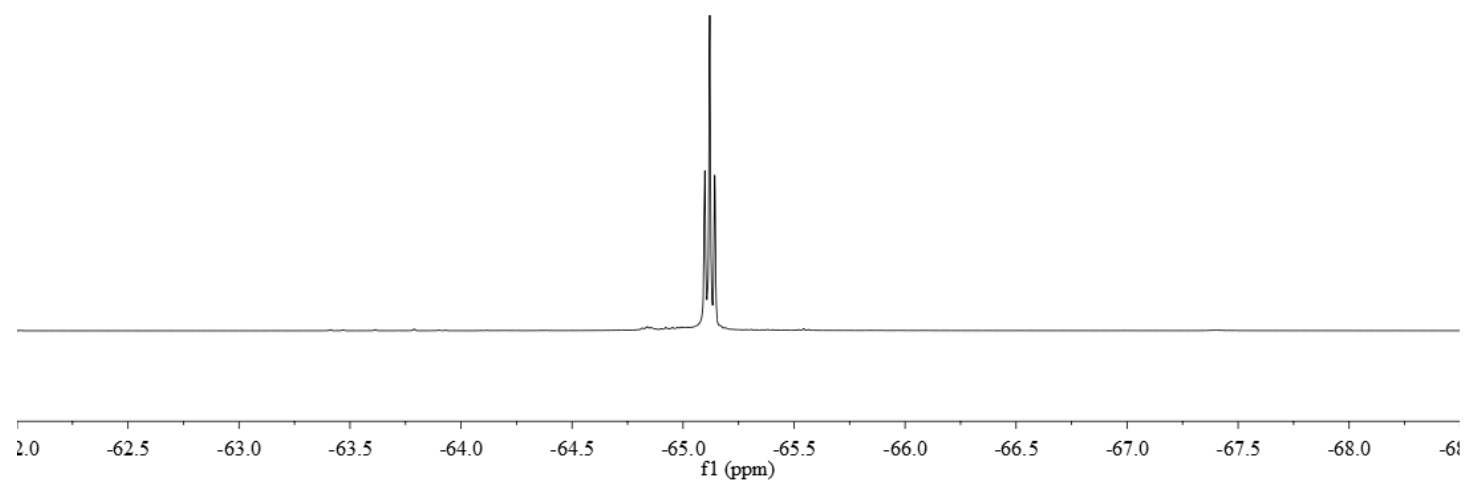




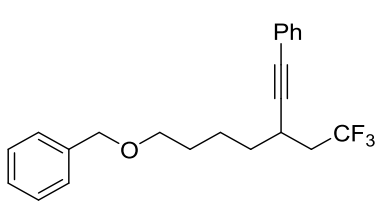

3t $\left(\mathrm{CDCl}_{3}, 101 \mathrm{MHz}\right)$

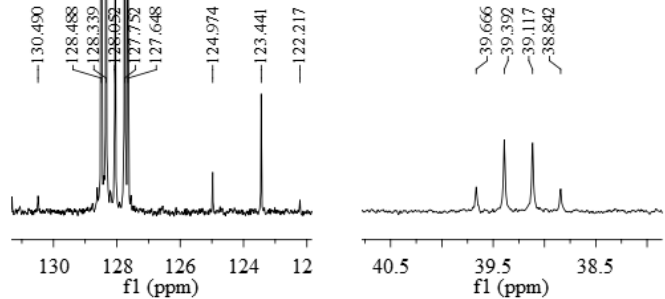

(ppm)
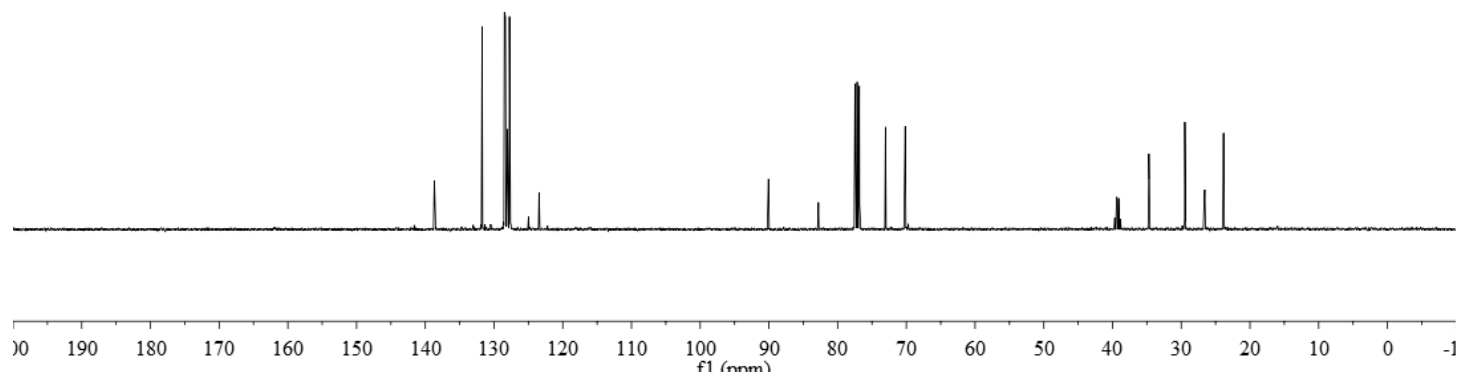

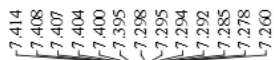

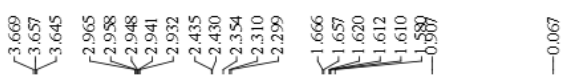

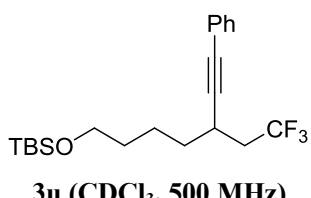

$3 \mathrm{u}\left(\mathrm{CDCl}_{3}, 500 \mathrm{MHz}\right)$

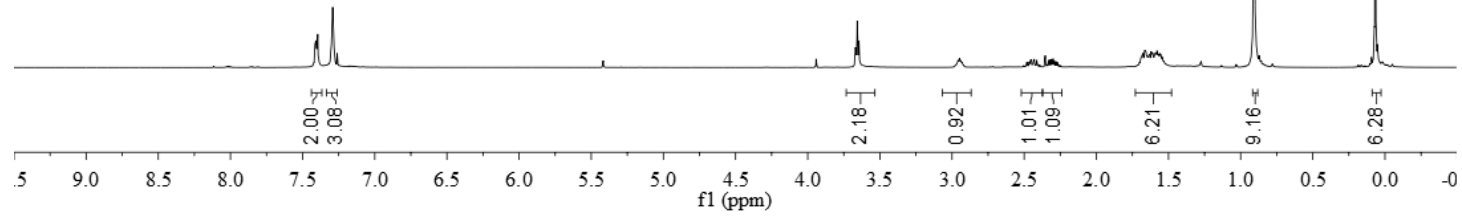




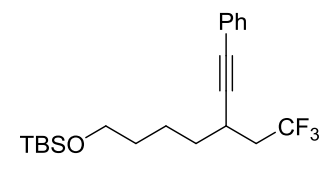

3u $\left(\mathrm{CDCl}_{3}, 470 \mathrm{MHz}\right)$

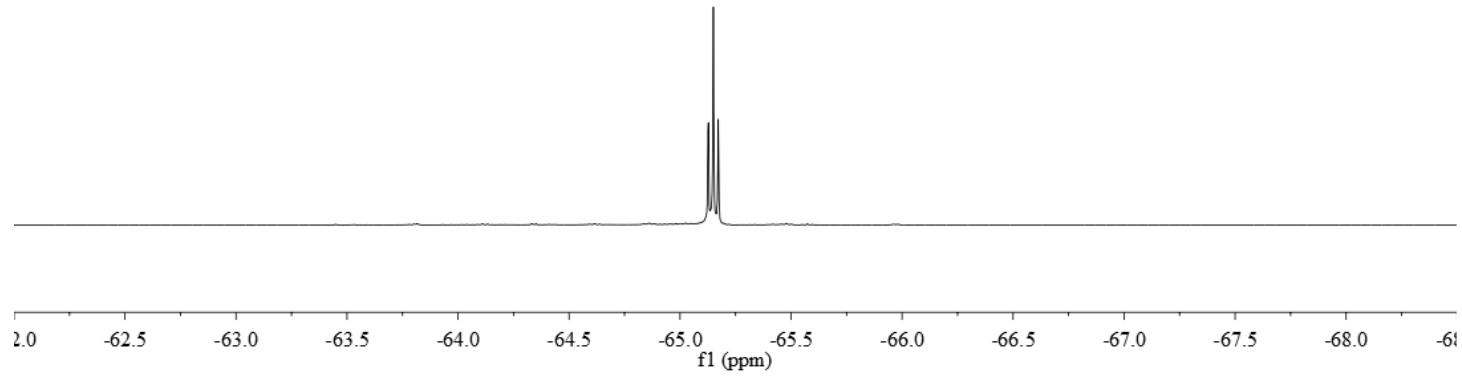

\section{항을 웡 \&}

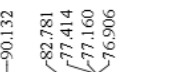

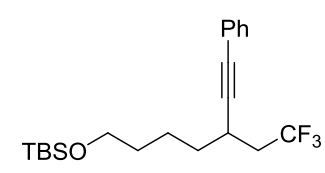

3u $\left(\mathrm{CDCl}_{3}, 101 \mathrm{MHz}\right)$
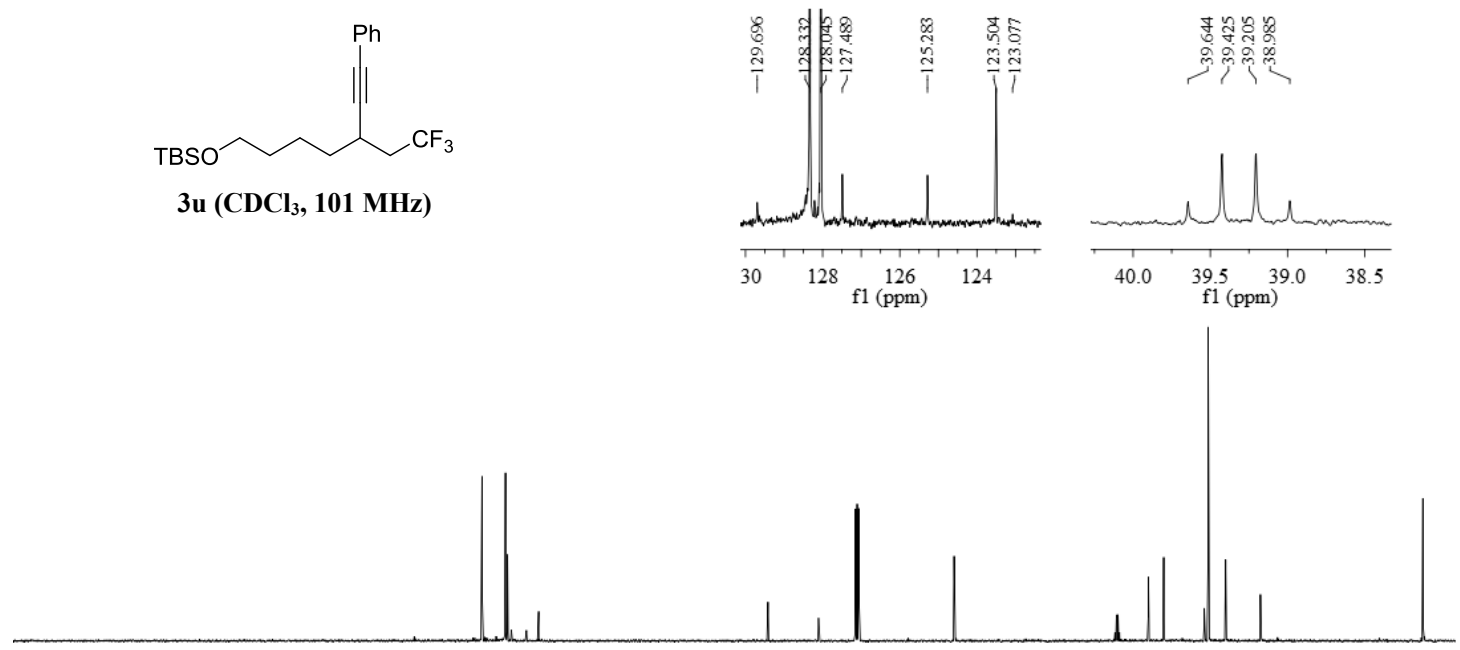

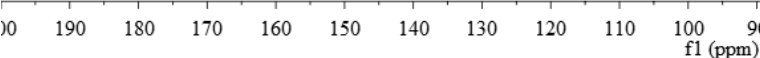




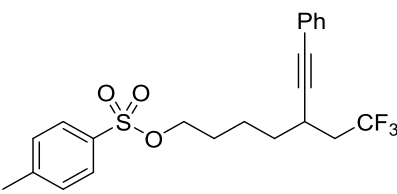

3v $\left(\mathrm{CDCl}_{3}, 400 \mathrm{MHz}\right)$

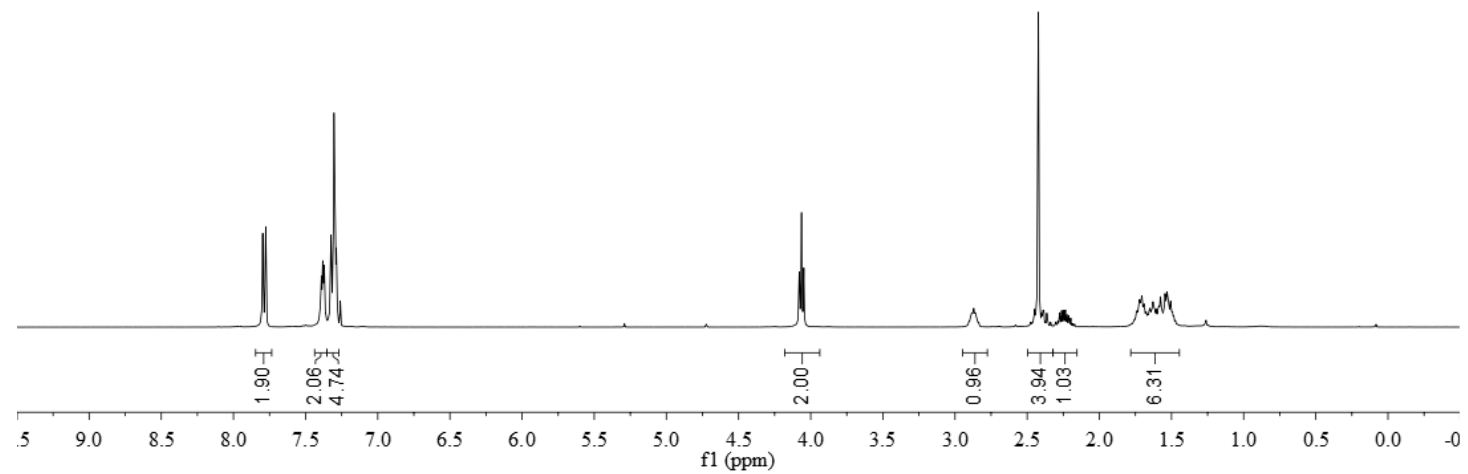

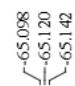

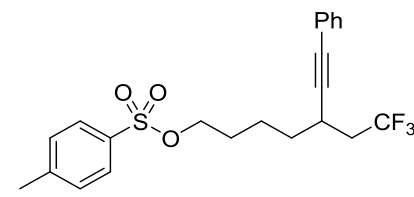

$3 \mathrm{v}\left(\mathrm{CDCl}_{3}, 470 \mathrm{MHz}\right)$

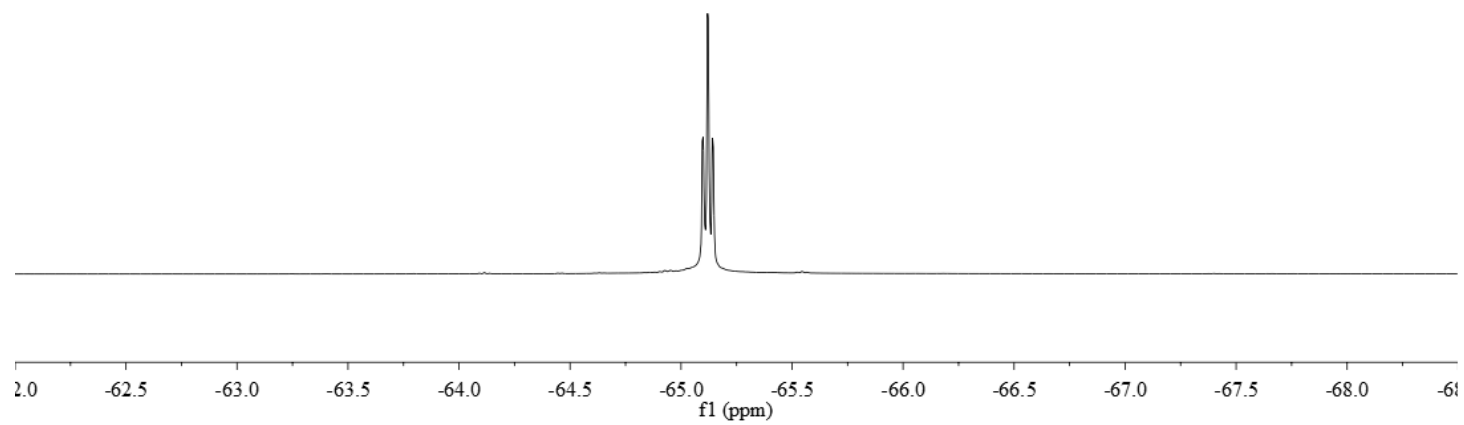




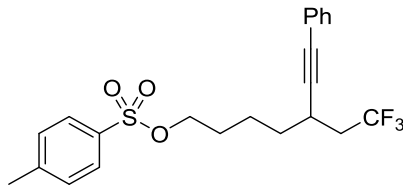

3v $\left(\mathrm{CDCl}_{3}, 101 \mathrm{MHz}\right)$
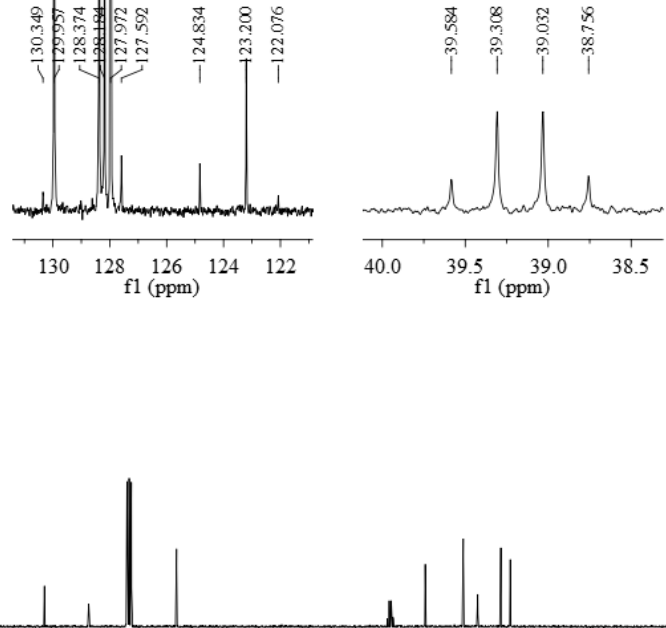

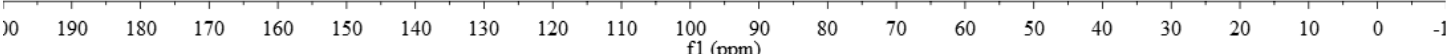

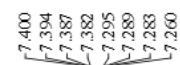

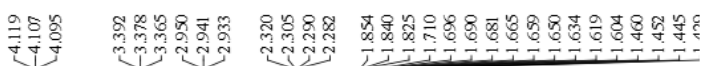<smiles>CC(F)(F)CC(C#CCCCC(C)(F)c1ccccc1)CCCCOC(=O)CCCCCBr</smiles>

3w $\left(\mathrm{CDCl}_{3}, 500 \mathrm{MHz}\right)$

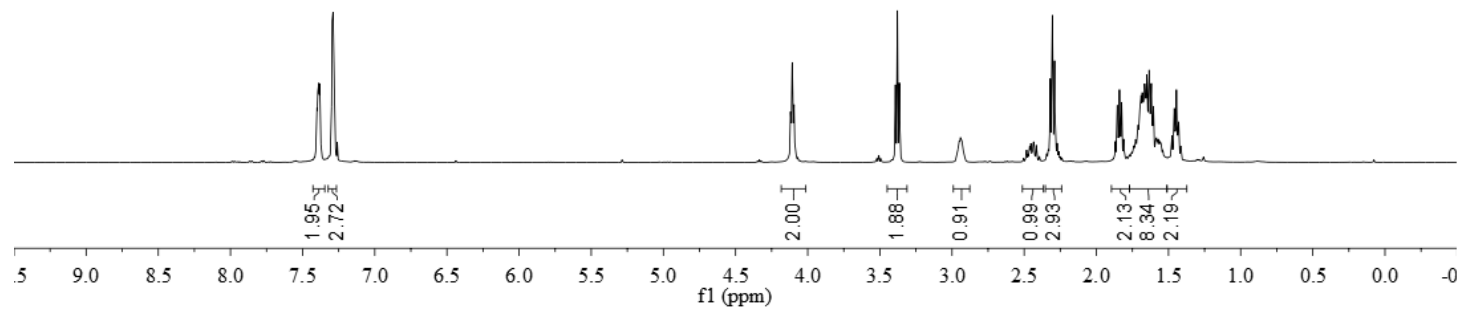




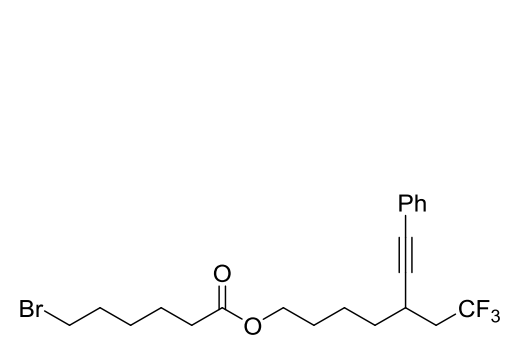

$3 \mathrm{w}\left(\mathrm{CDCl}_{3}, 470 \mathrm{MHz}\right)$

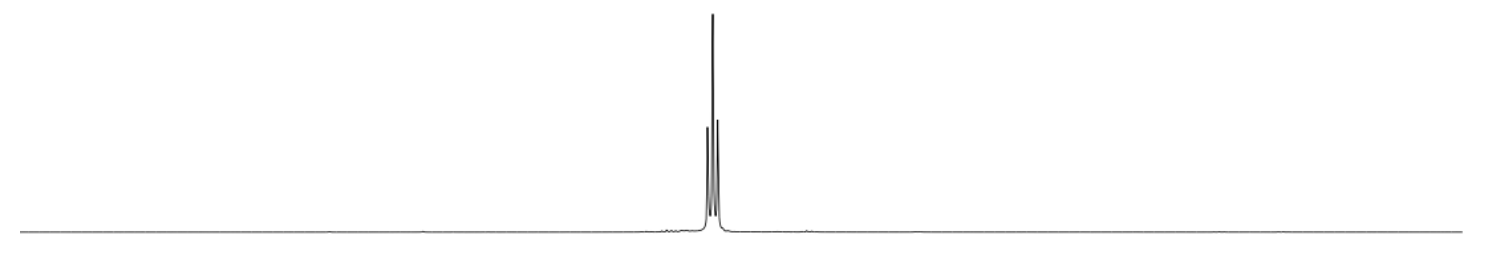

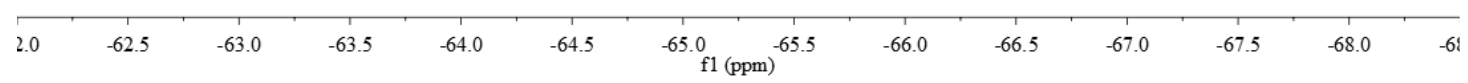
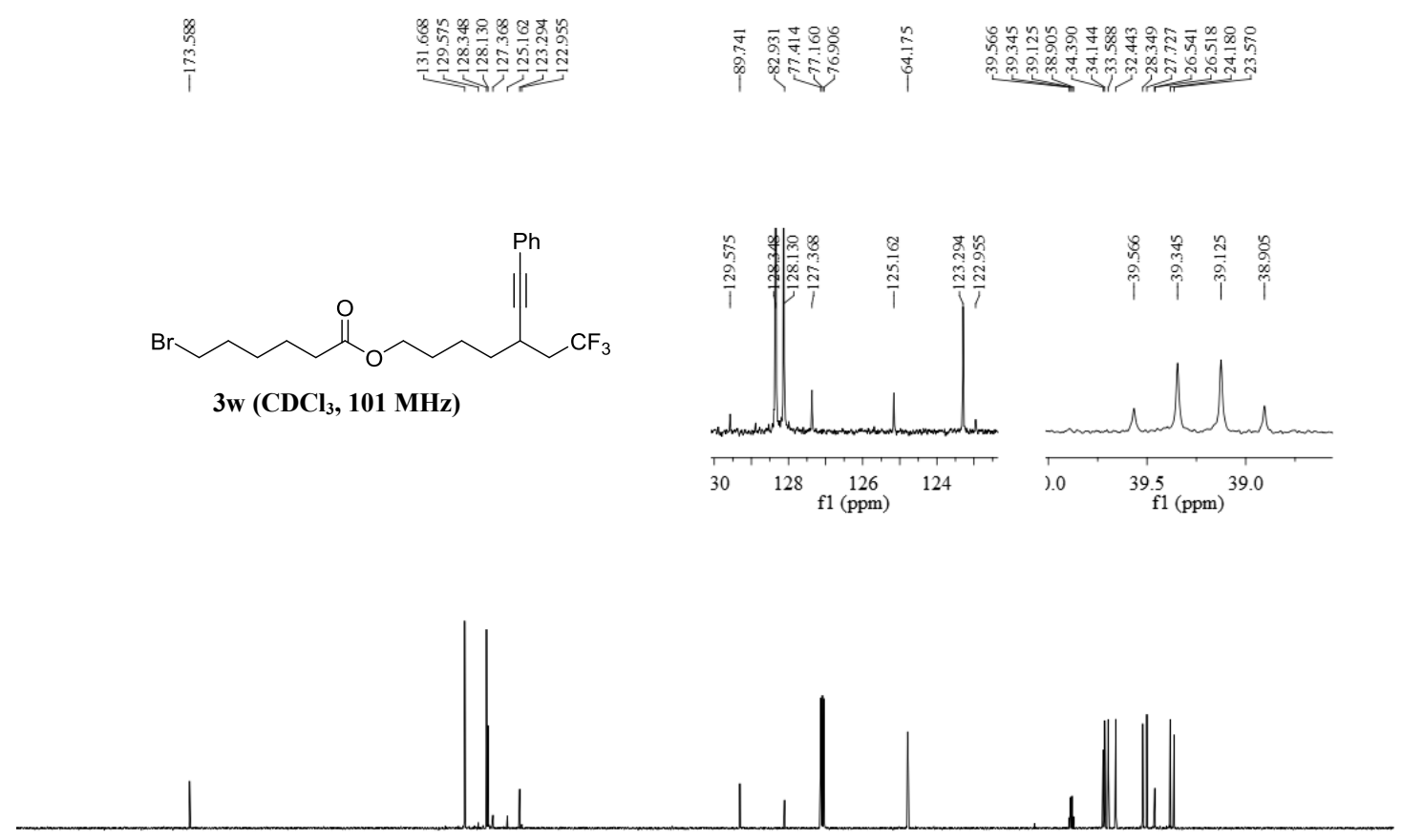

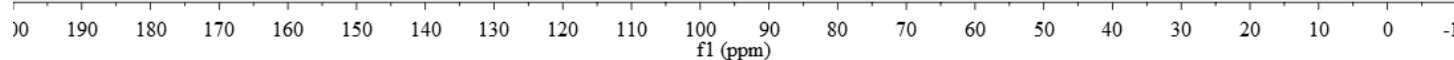




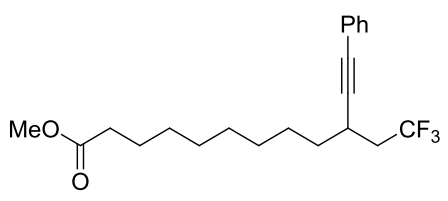

3x $\left(\mathrm{CDCl}_{3}, 500 \mathrm{MHz}\right)$

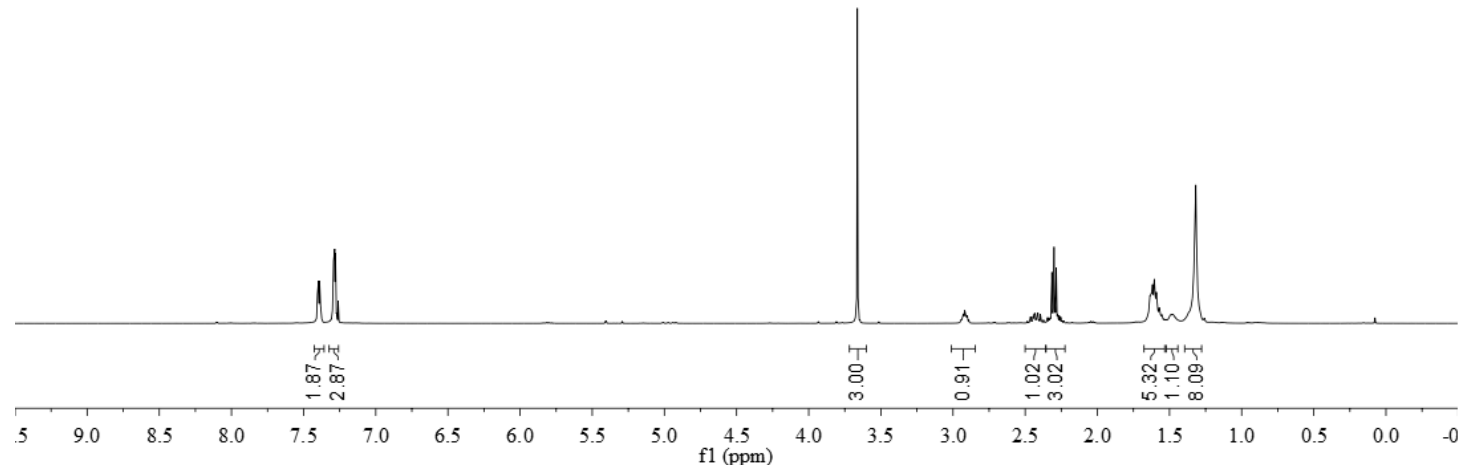

ले 80

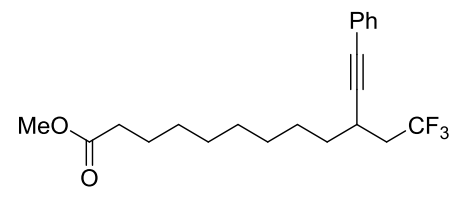

3x $\left(\mathrm{CDCl}_{3}, 470 \mathrm{MHz}\right)$

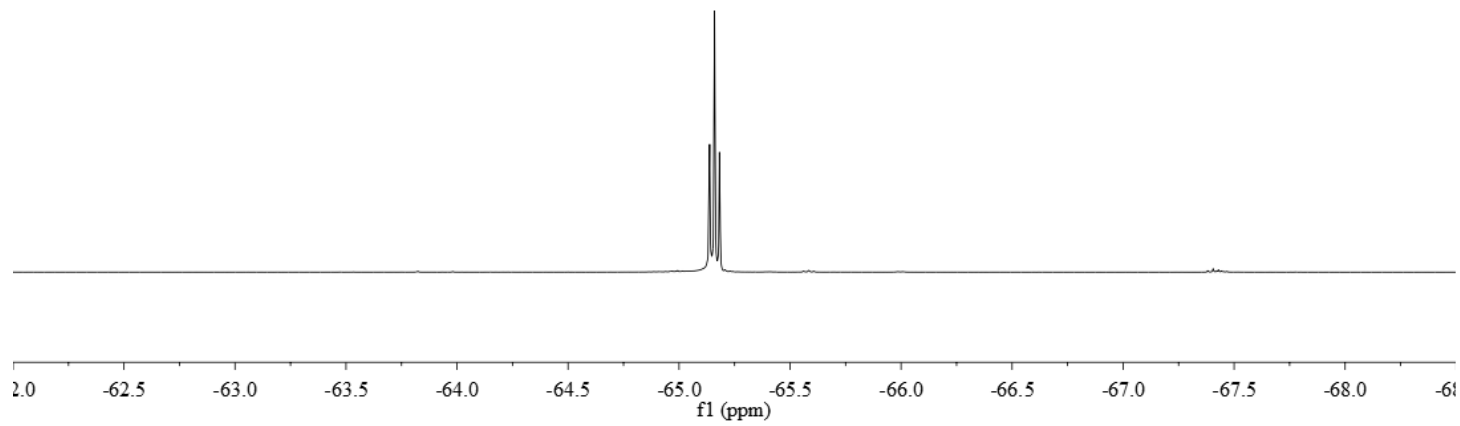




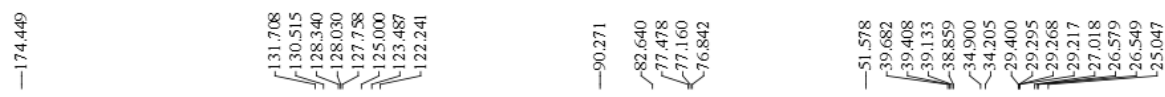

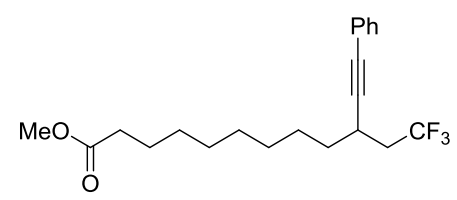

3x $\left(\mathrm{CDCl}_{3}, 101 \mathrm{MHz}\right)$

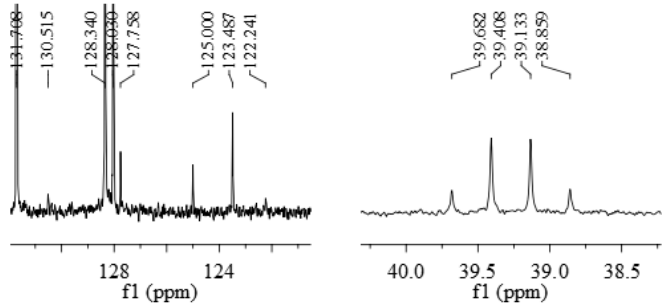

$1(\mathrm{ppm})$

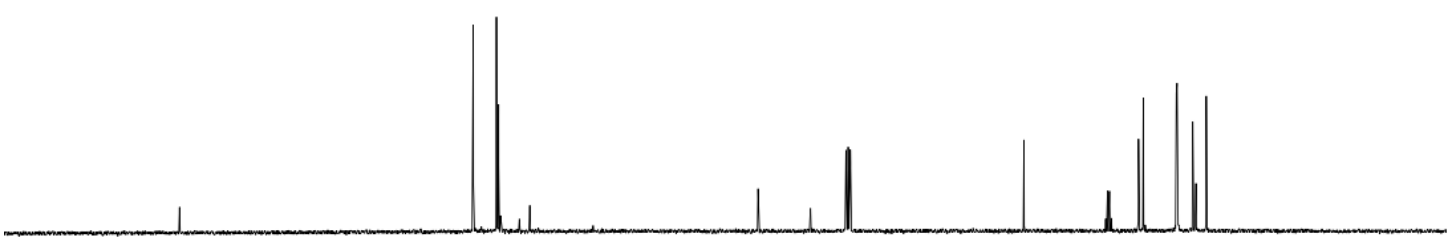

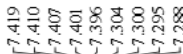

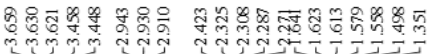
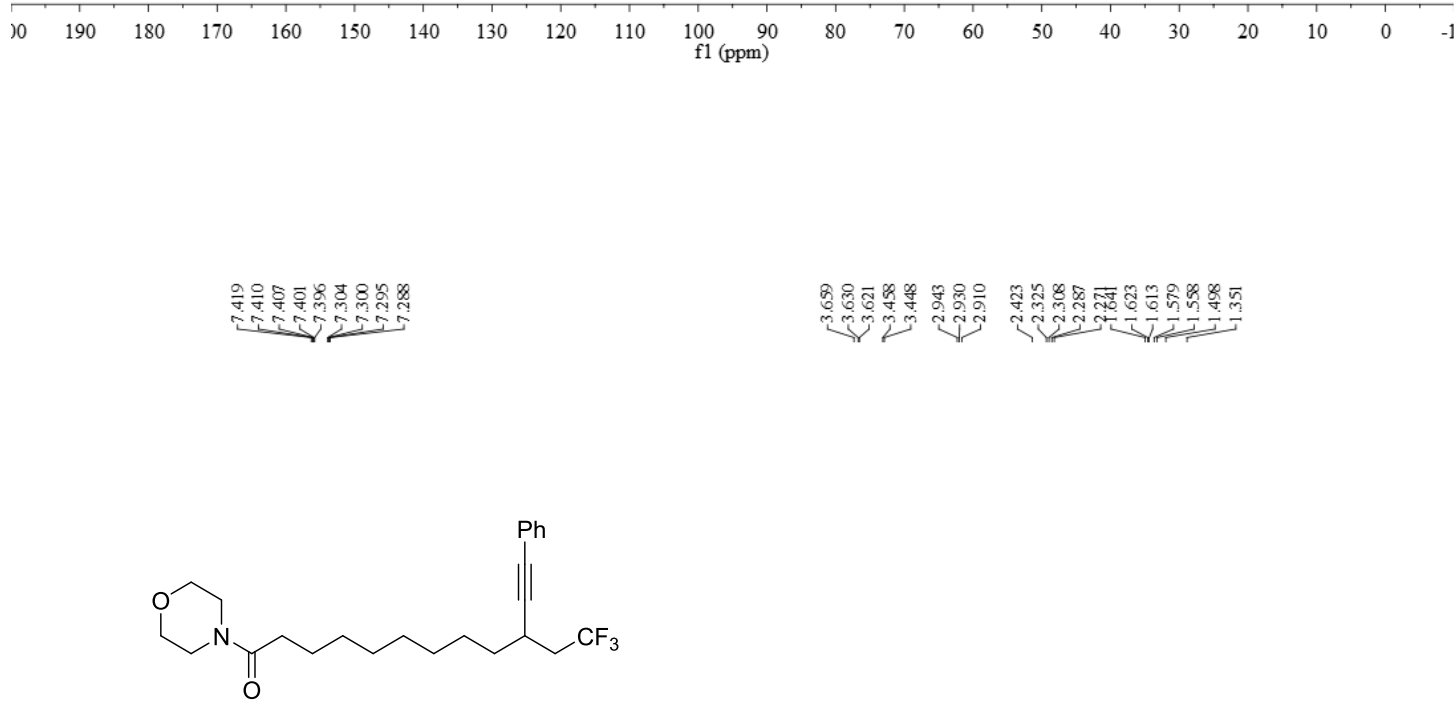

$3 y\left(\mathrm{CDCl}_{3}, 400 \mathrm{MHz}\right)$

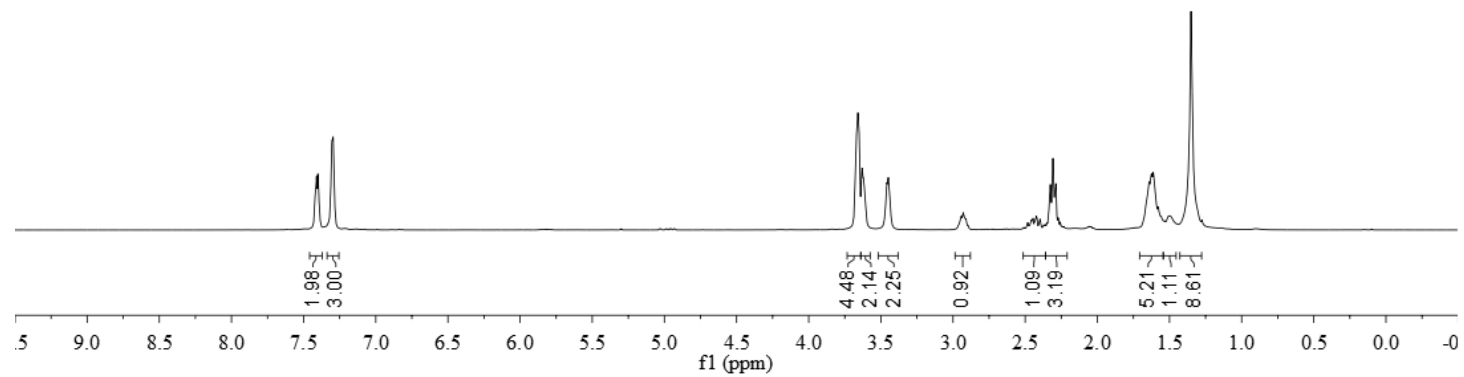




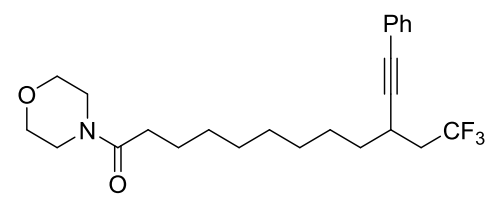

$3 y\left(\mathrm{CDCl}_{3}, 470 \mathrm{MHz}\right)$
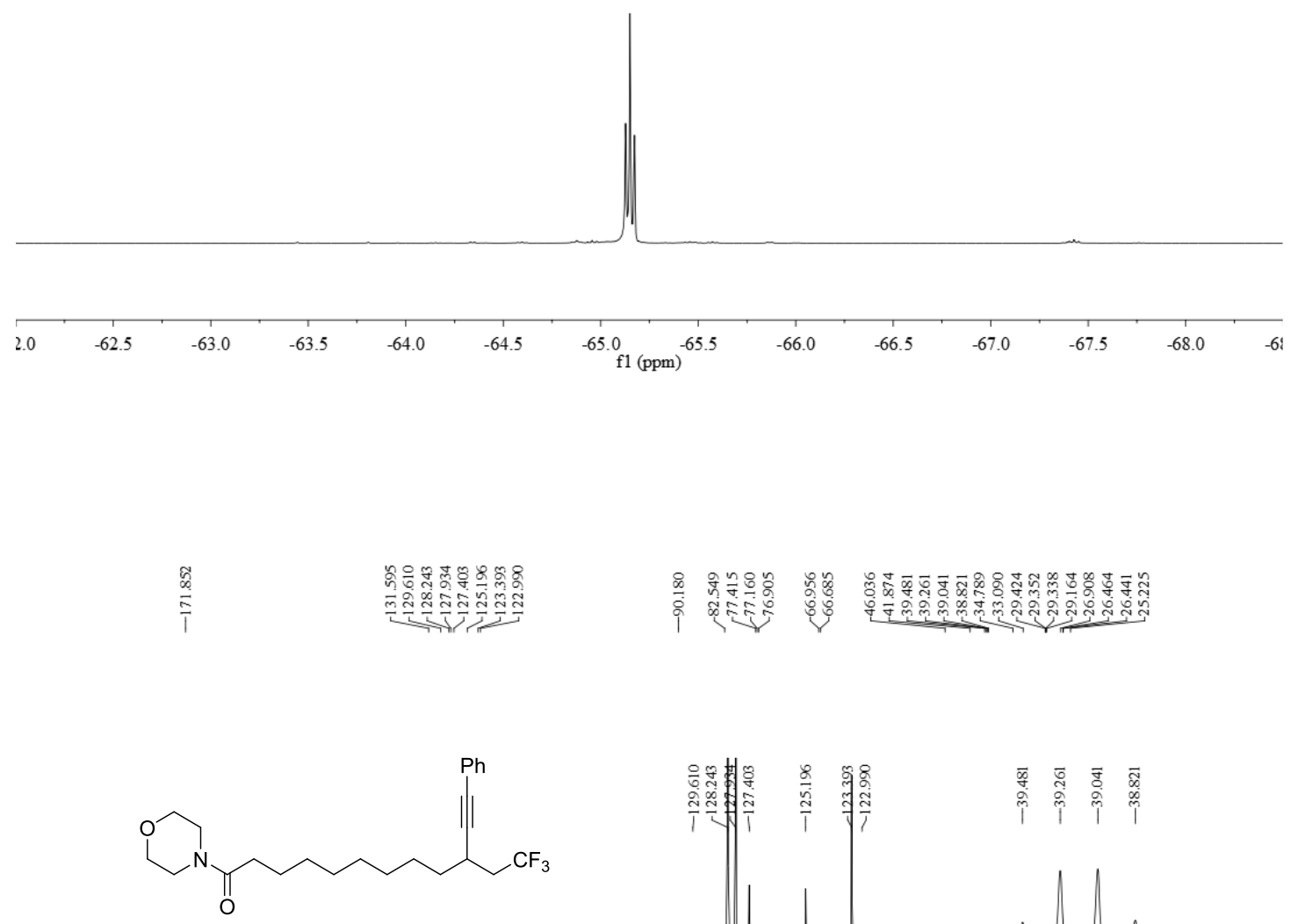

$3 y\left(\mathrm{CDCl}_{3}, 126 \mathrm{MHz}\right)$
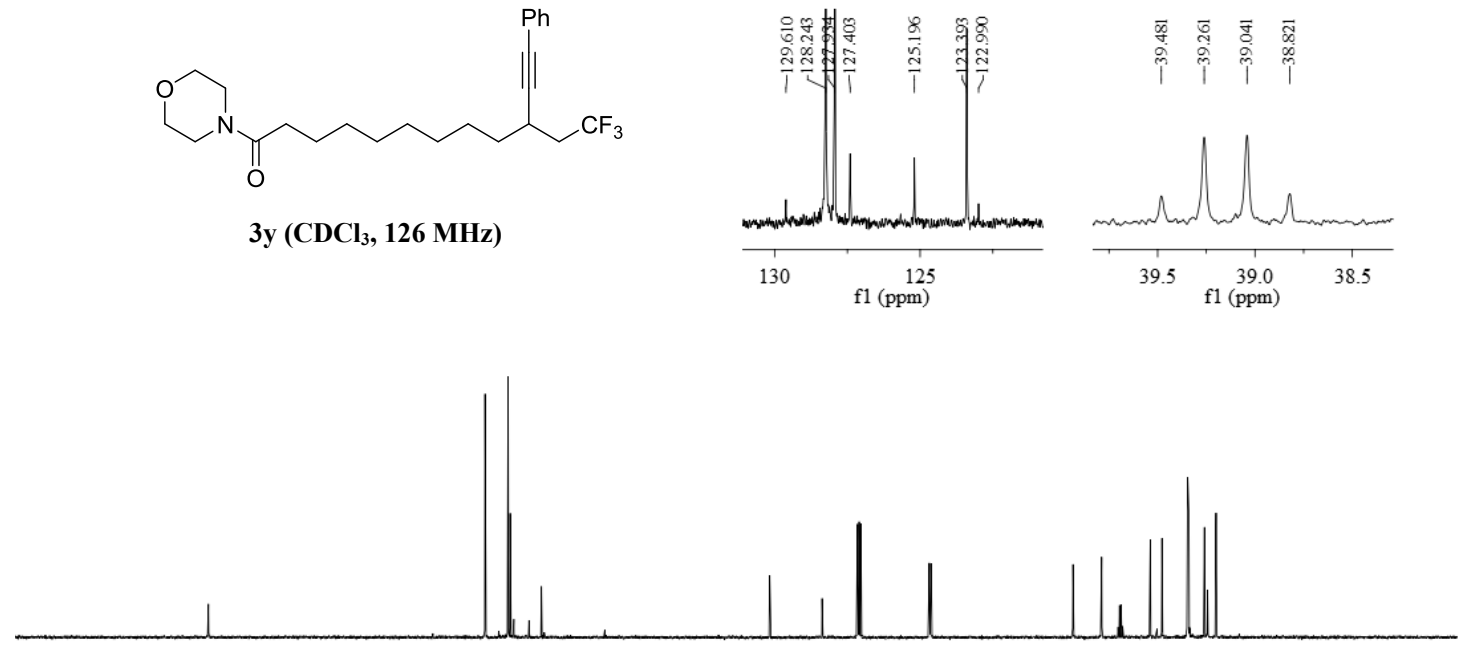

$\begin{array}{lllllllllll}10 & 190 & 180 & 170 & 160 & 150 & 140 & 130 & 120 & 110 & 100 \\ \mathrm{f} 1(\mathrm{ppm})\end{array}$ 


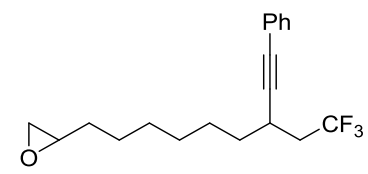

$3 \mathrm{z}\left(\mathrm{CDCl}_{3}, 500 \mathrm{MHz}\right)$
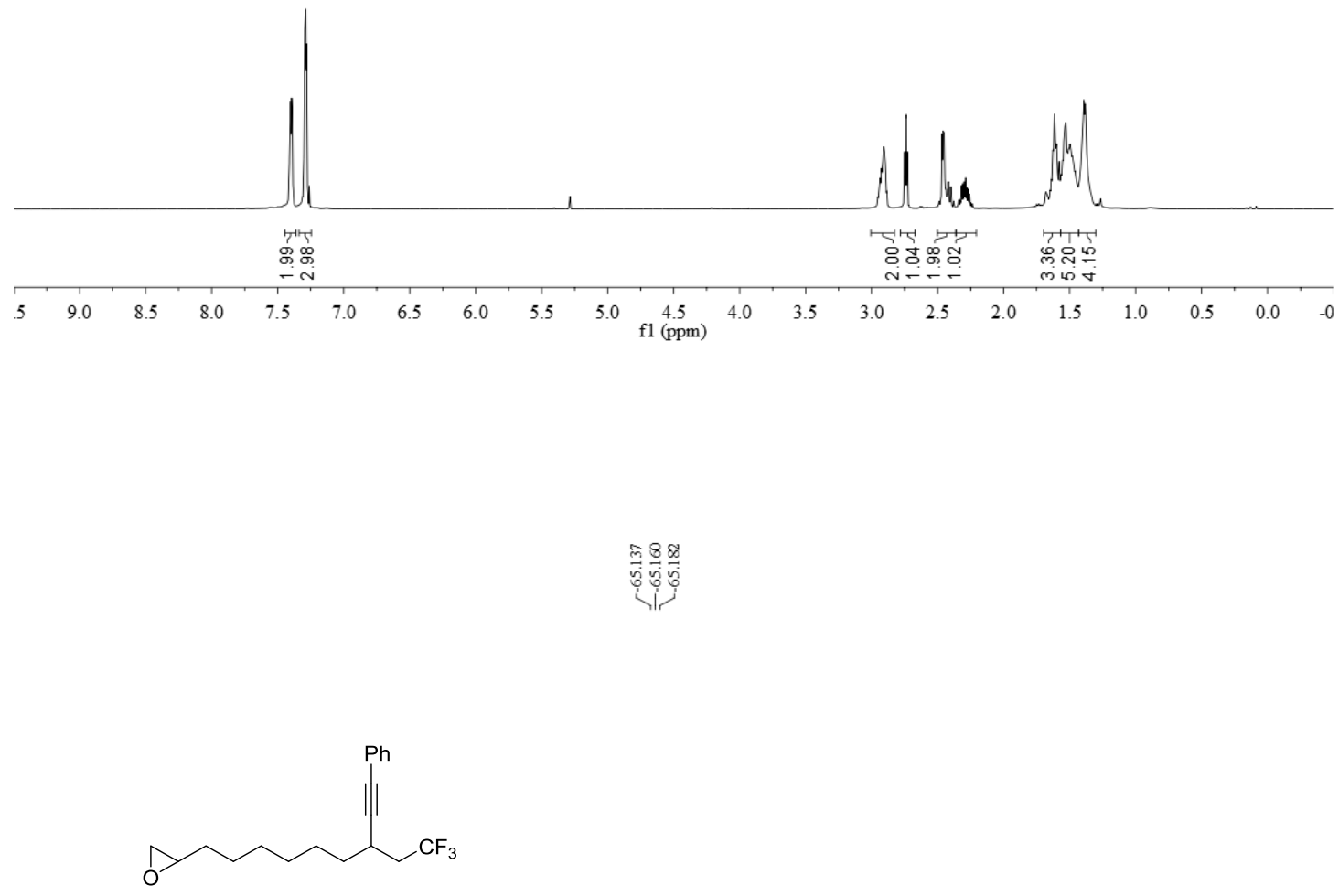

$3 \mathrm{z}\left(\mathrm{CDCl}_{3}, 470 \mathrm{MHz}\right)$

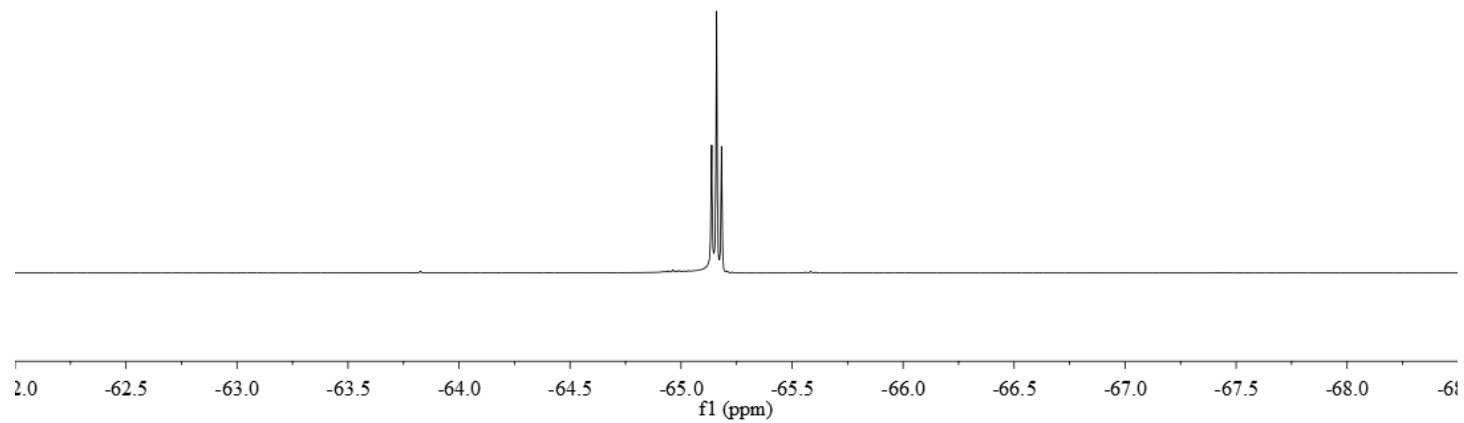




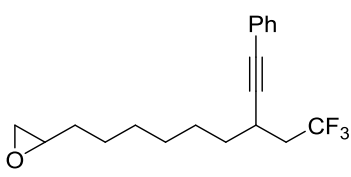

$3 \mathrm{z}\left(\mathrm{CDCl}_{3}, 101 \mathrm{MHz}\right)$

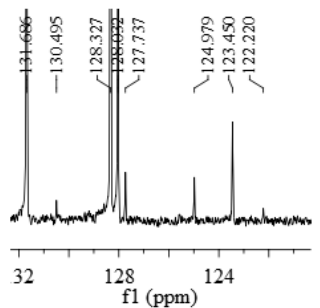

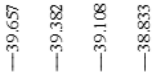

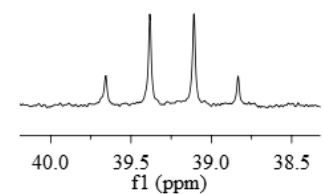

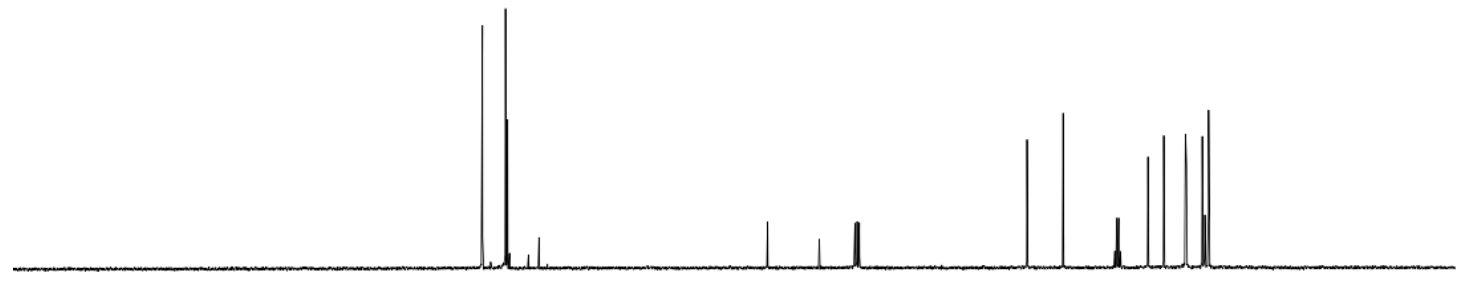

$\begin{array}{llllllllllllllllllllllllll}10 & 190 & 180 & 170 & 160 & 150 & 140 & 130 & 120 & 110 & 100 & 90 & 80 & 70 & 60 & 50 & 40 & 30 & 20 & 10 & 0 & -1\end{array}$

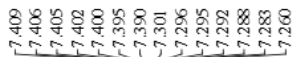

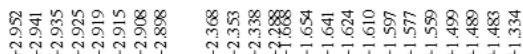

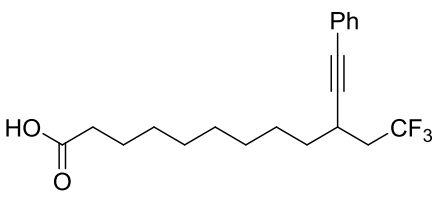

3aa $\left(\mathrm{CDCl}_{3}, 500 \mathrm{MHz}\right)$

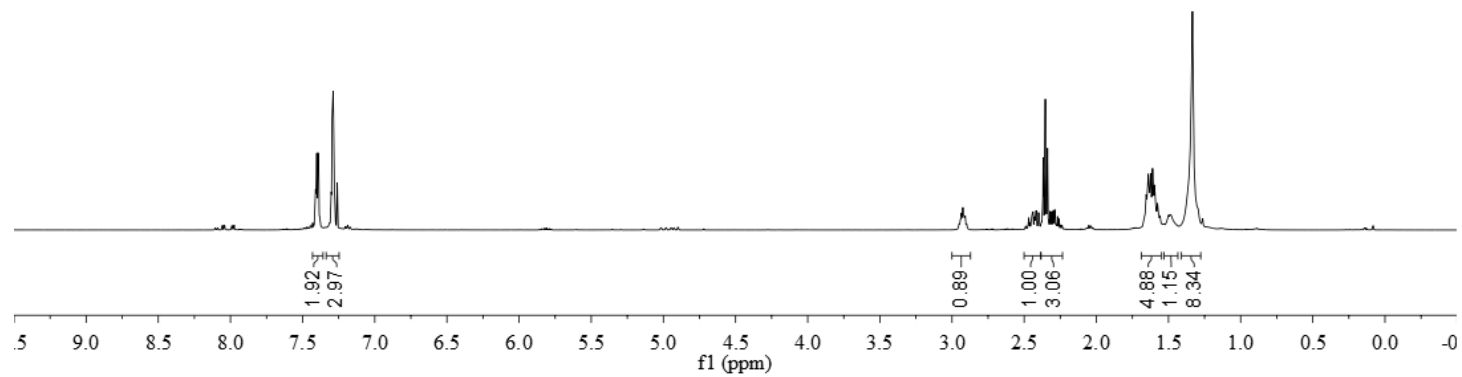



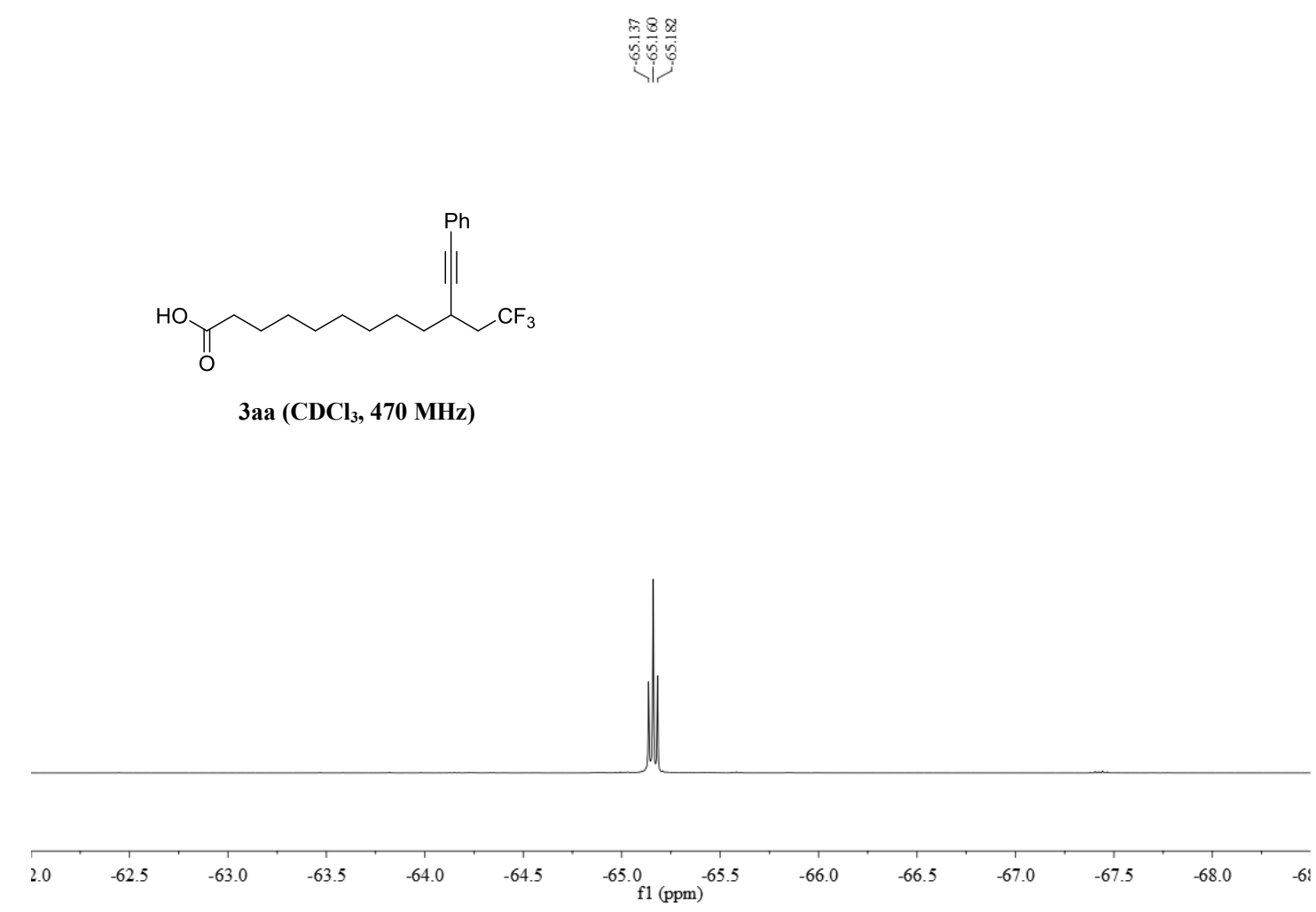

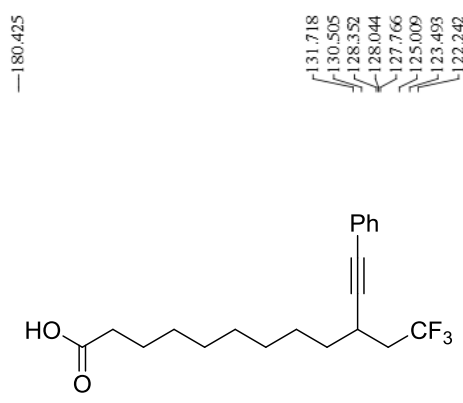

3aa $\left(\mathrm{CDCl}_{3}, 101 \mathrm{MHz}\right)$
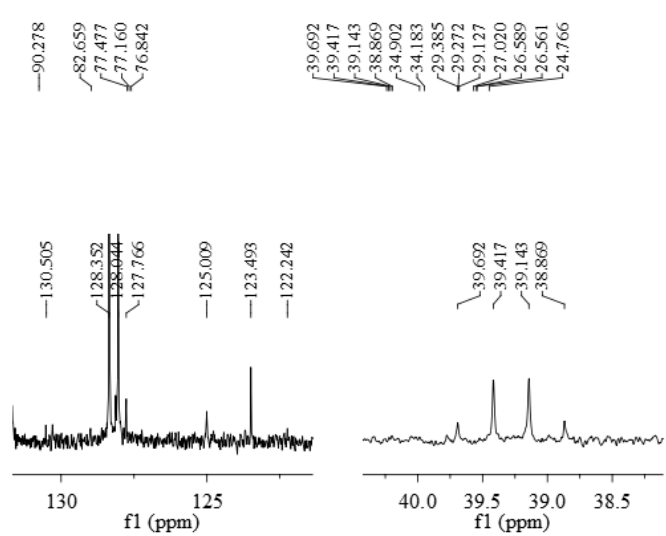

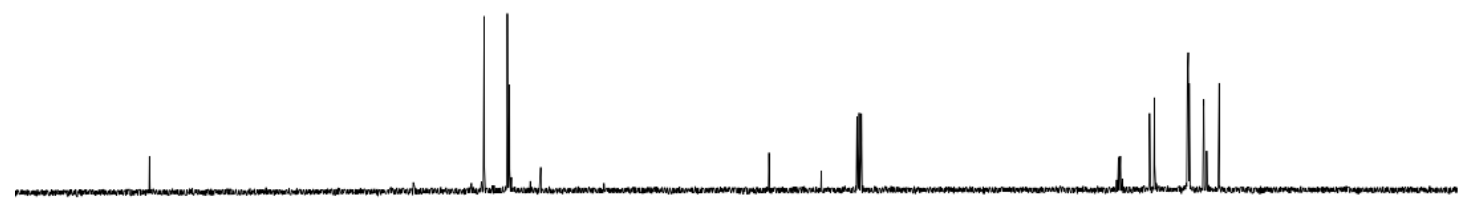

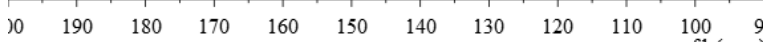




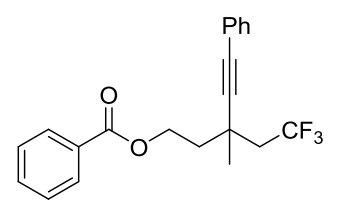

$3 \mathrm{ab}\left(\mathrm{CDCl}_{3}, 400 \mathrm{MHz}\right)$

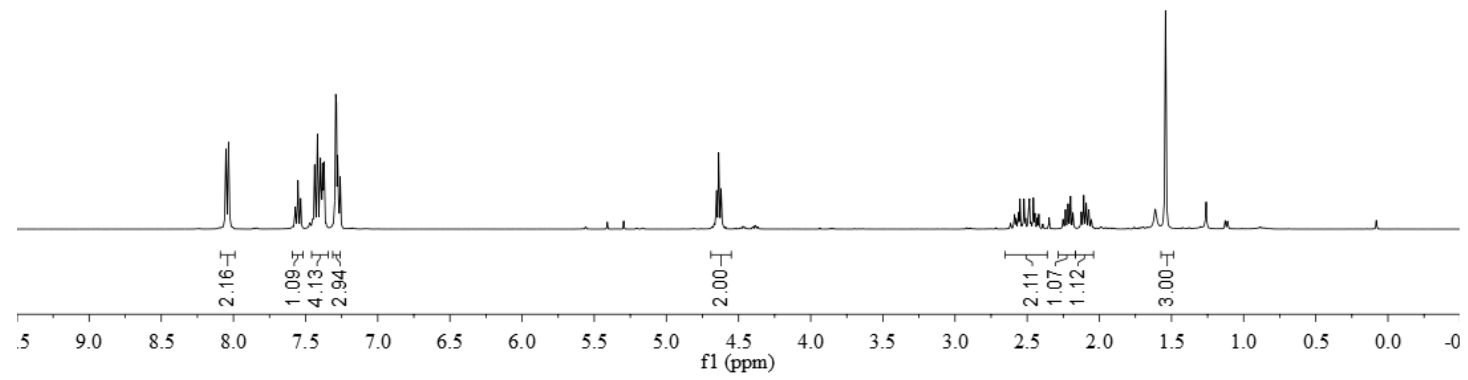

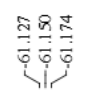

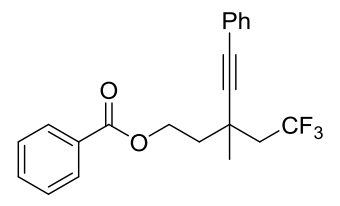

$3 \mathrm{ab}\left(\mathrm{CDCl}_{3}, 470 \mathrm{MHz}\right)$

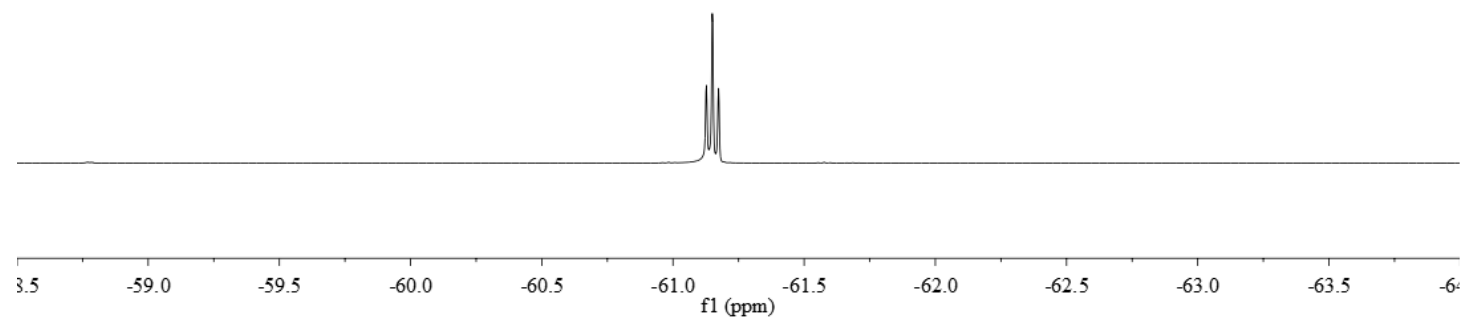


<smiles>CC(C#Cc1ccccc1)(CCOC(=O)c1ccccc1)CC(F)(F)F</smiles>

3ab $\left(\mathrm{CDCl}_{3}, 101 \mathrm{MHz}\right)$
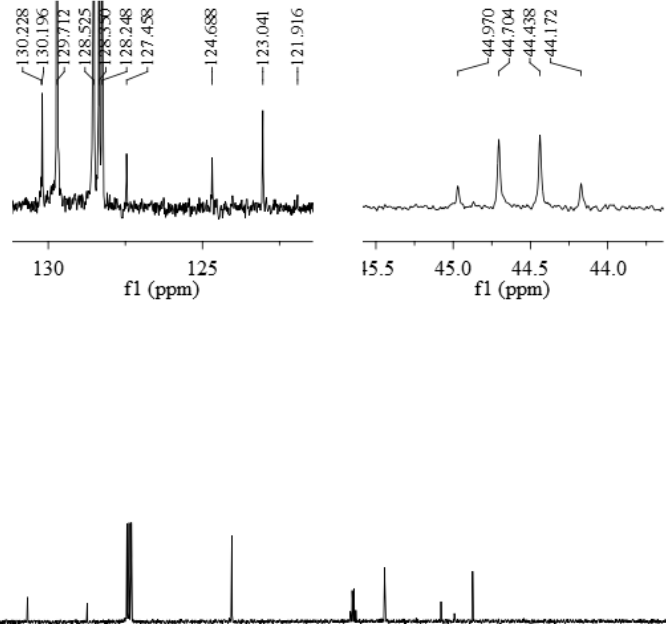

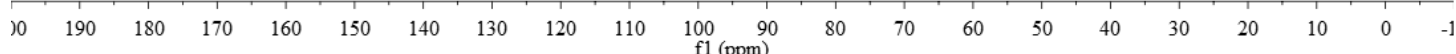

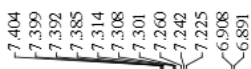

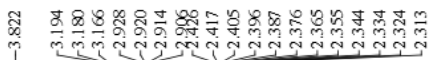

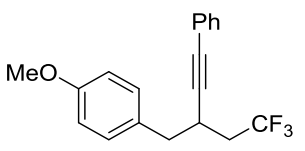

3ac $\left(\mathrm{CDCl}_{3}, 500 \mathrm{MHz}\right)$

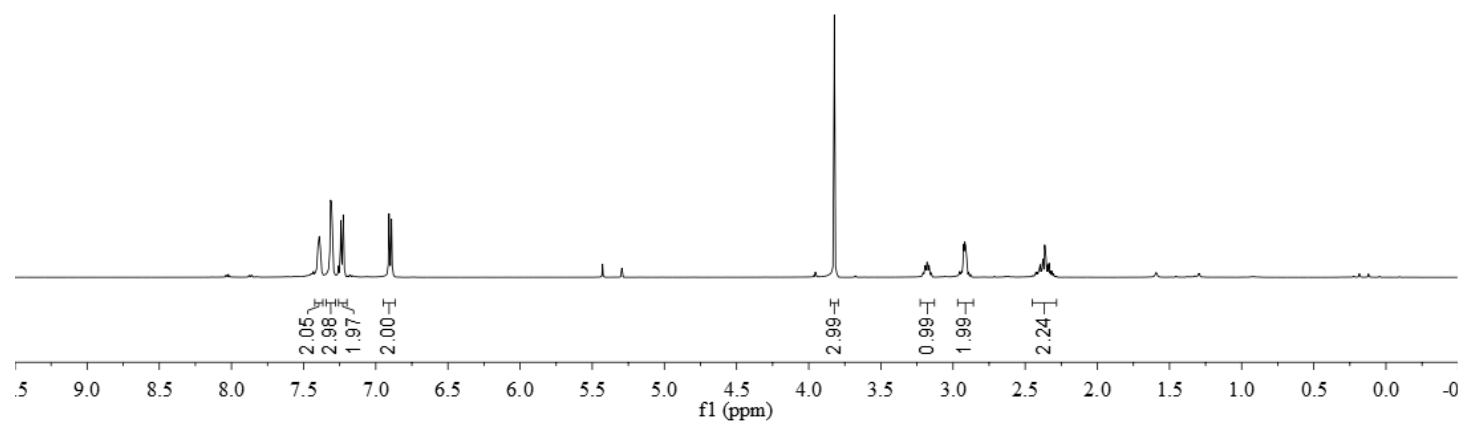




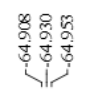
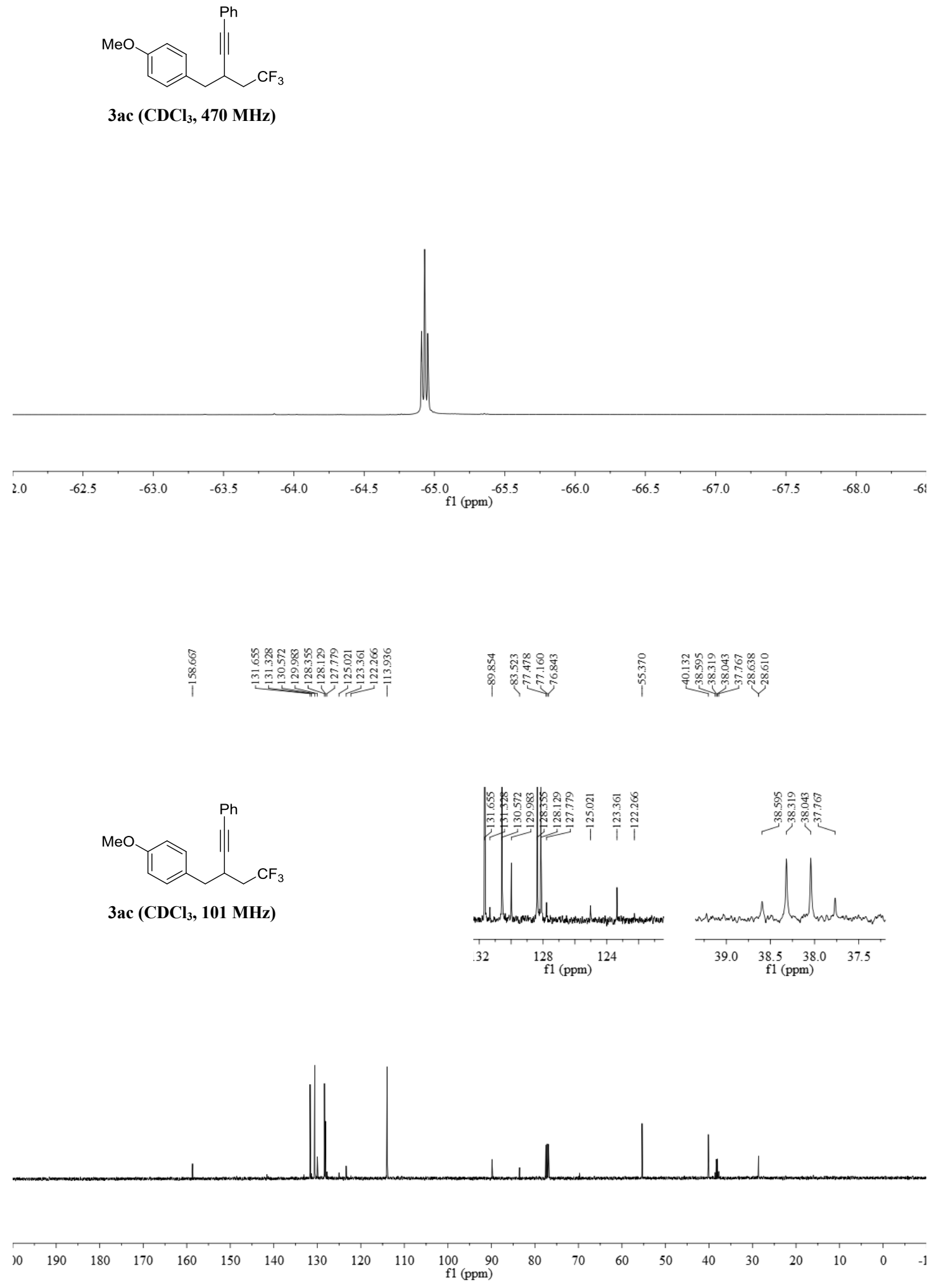

S80 


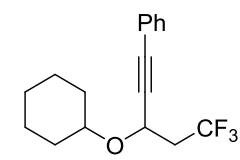

$\operatorname{3ad}\left(\mathrm{CDCl}_{3}, 500 \mathrm{MHz}\right)$

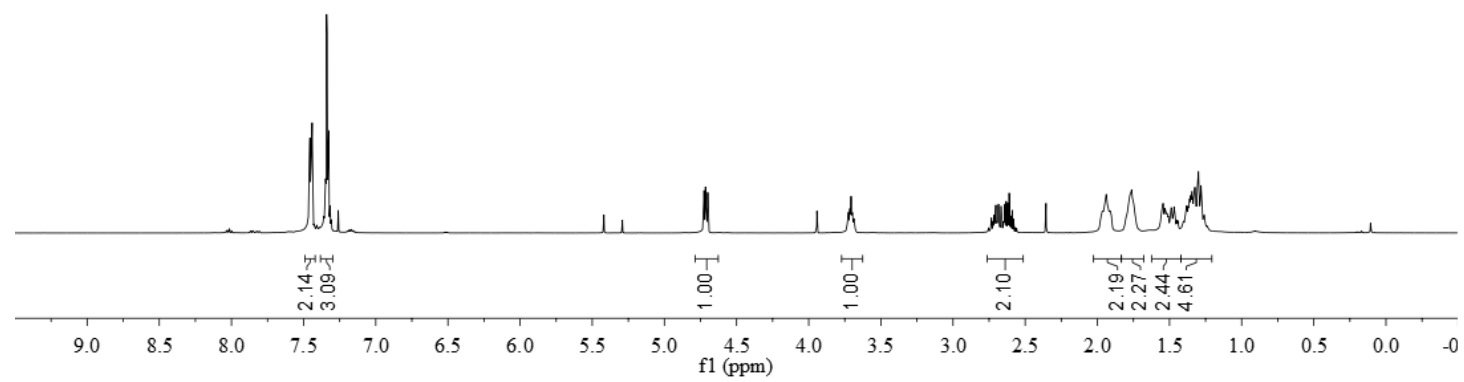

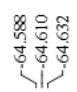<smiles>FC(F)(F)CC(C#Cc1ccccc1)OC1CCCCC1</smiles>

$\operatorname{3ad}\left(\mathrm{CDCl}_{3}, 470 \mathrm{MHz}\right)$

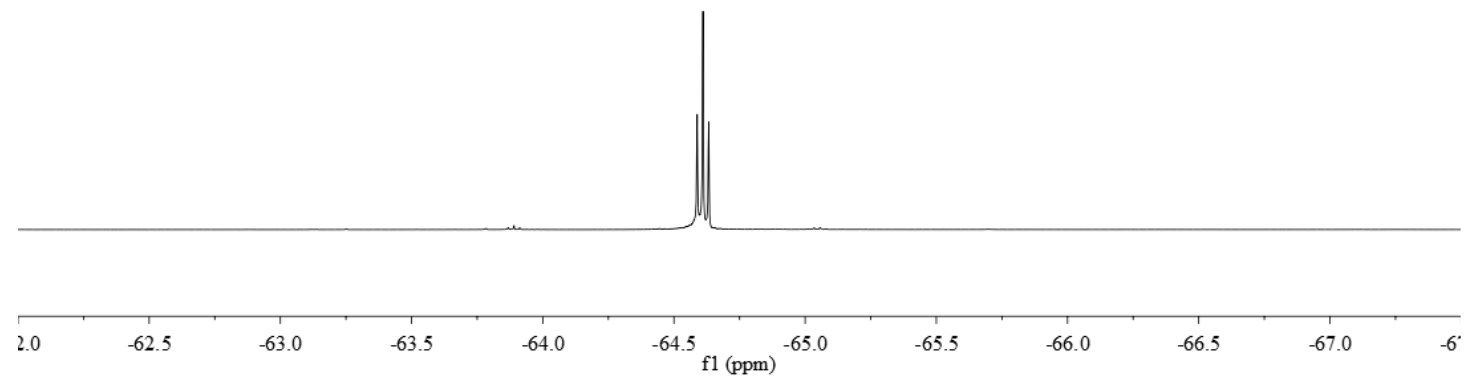




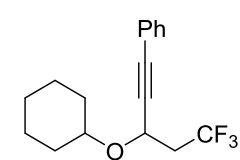

$\operatorname{3ad}\left(\mathrm{CDCl}_{3}, 101 \mathrm{MHz}\right)$

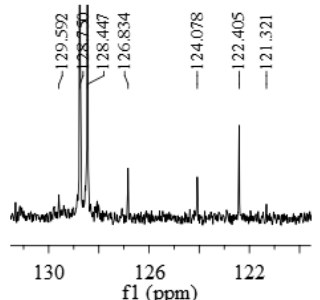

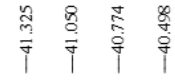

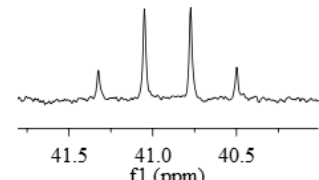

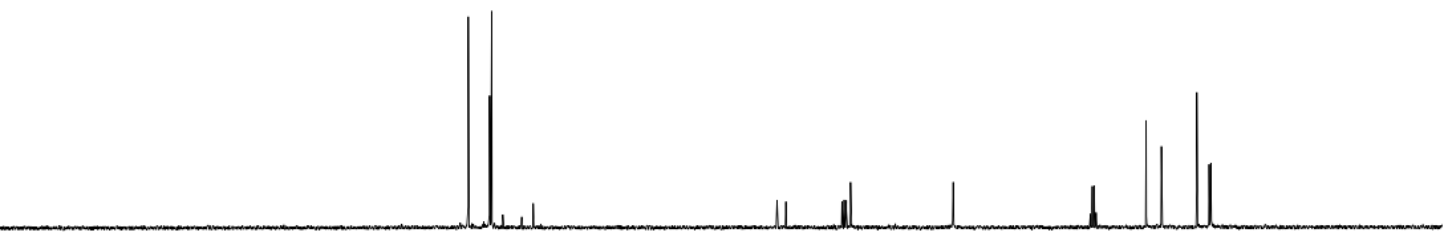

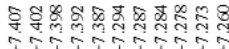

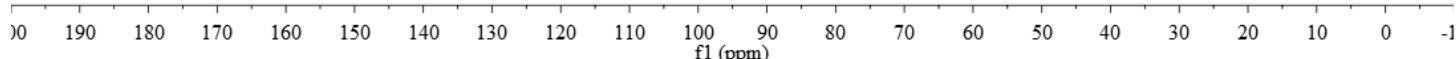<smiles>FC(F)(F)C1CCCC[C@@H]1C#Cc1ccccc1</smiles>

3 ae $\left(\mathrm{CDCl}_{3}, 500 \mathrm{MHz}\right)$

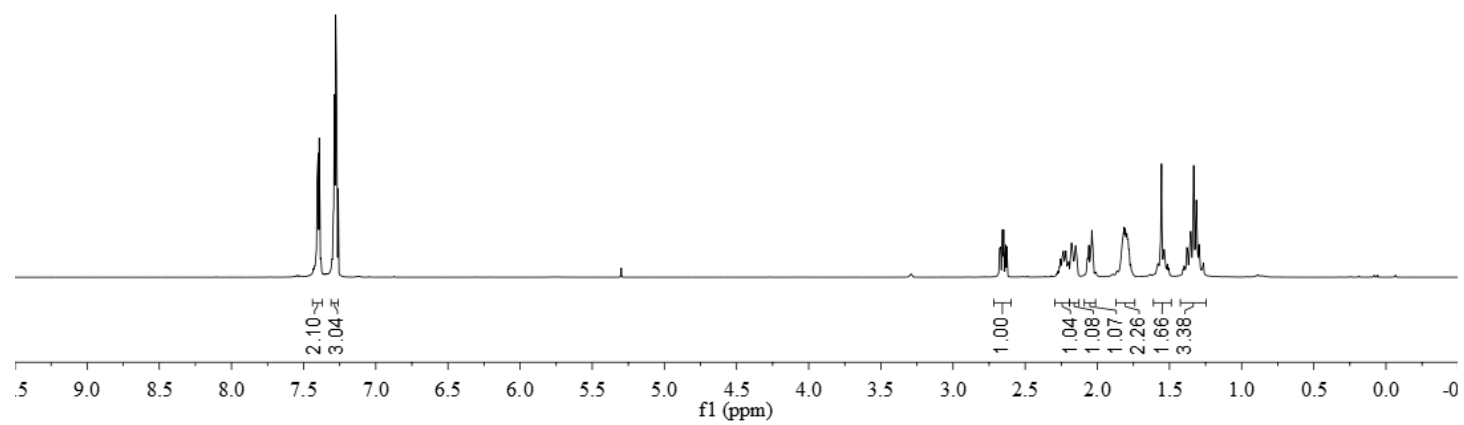




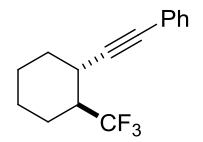

3ae (CDCl 3 , $470 \mathrm{MHz})$

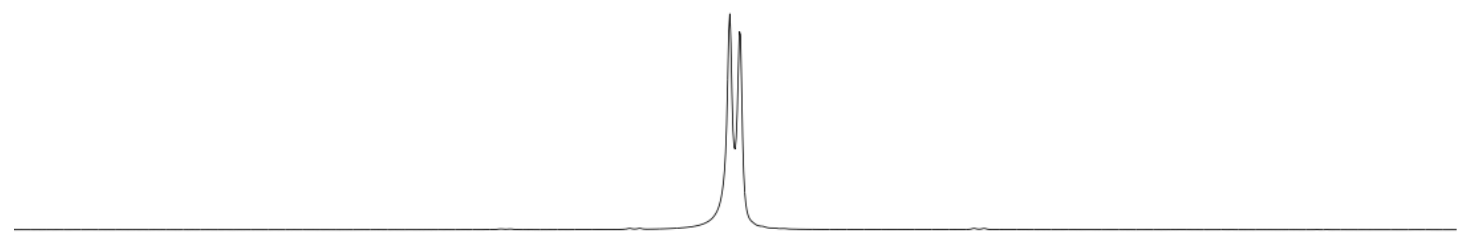

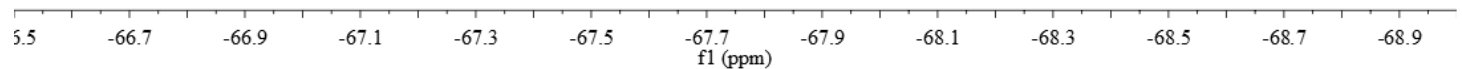

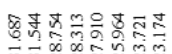

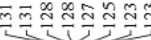

$\infty$
$\infty$
0
$\infty$

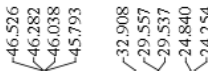
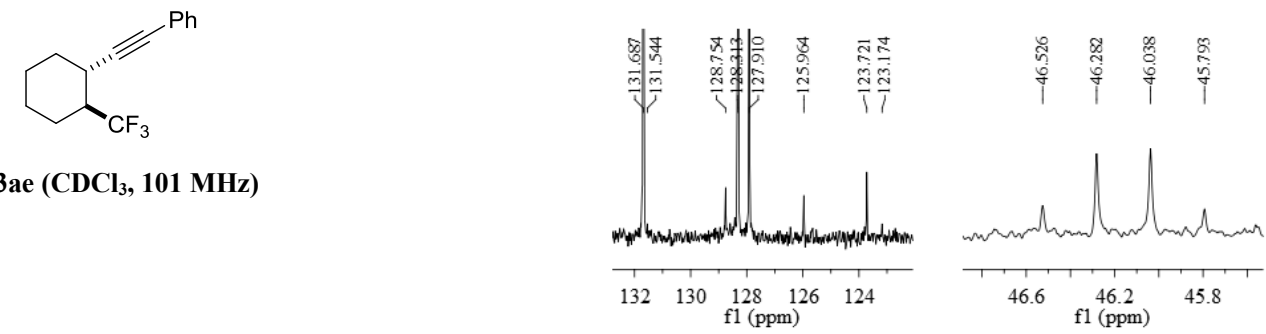

3ae $\left(\mathrm{CDCl}_{3}, 101 \mathrm{MHz}\right)$

$\begin{array}{llll}132 & 130 & 128 & 126 \\ \mathrm{f} 1(\mathrm{ppm}) & 12\end{array}$ $\begin{array}{lcc}46.6 & 46.2 & 45.8\end{array}$

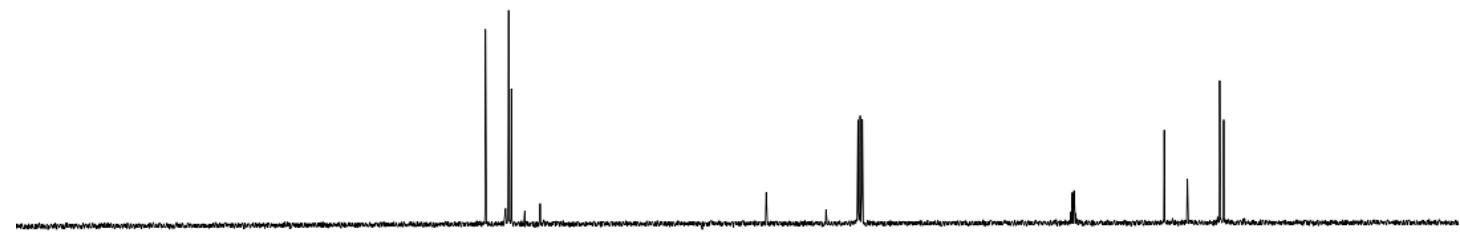

$\begin{array}{lllllllllll}10 & 190 & 180 & 170 & 160 & 150 & 140 & 130 & 120 & 110 & 100\end{array}$ 1 (ppm) 


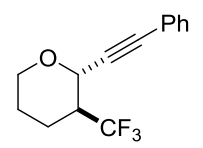

3af $\left(\mathrm{CDCl}_{3}, 400 \mathrm{MHz}\right)$

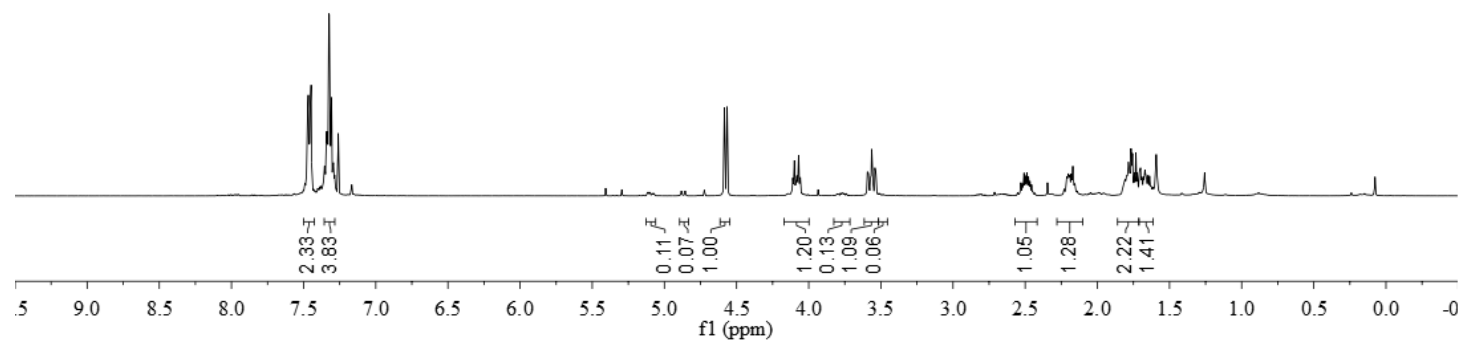

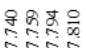

웅

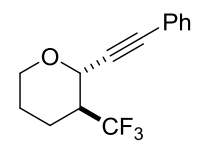

3af $\left(\mathrm{CDCl}_{3}, 470 \mathrm{MHz}\right)$

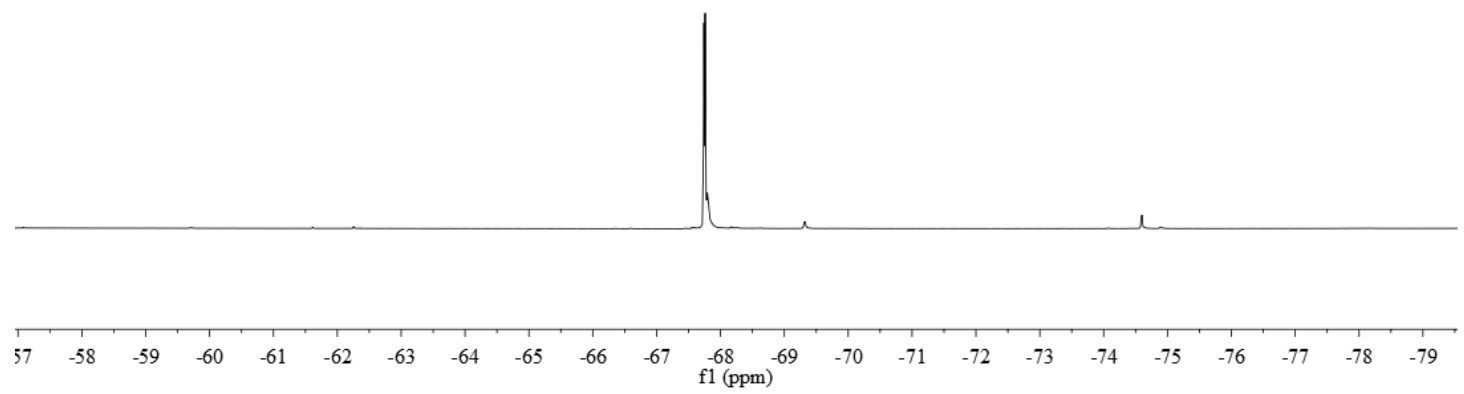




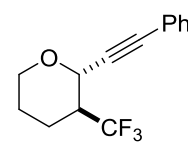

3af $\left(\mathrm{CDCl}_{3}, 126 \mathrm{MHz}\right)$
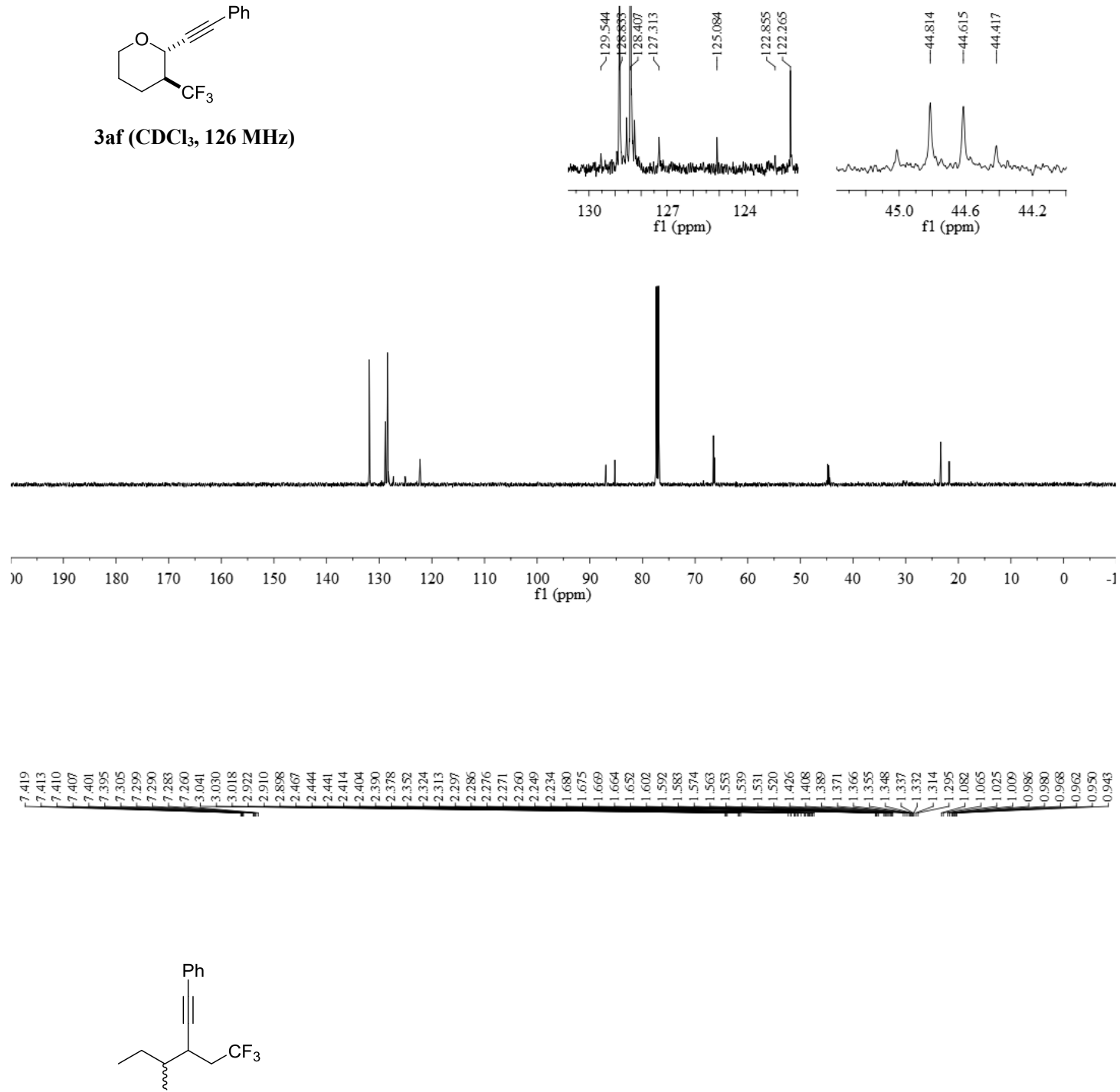

3ag $\left(\mathrm{CDCl}_{3}, 400 \mathrm{MHz}\right)$

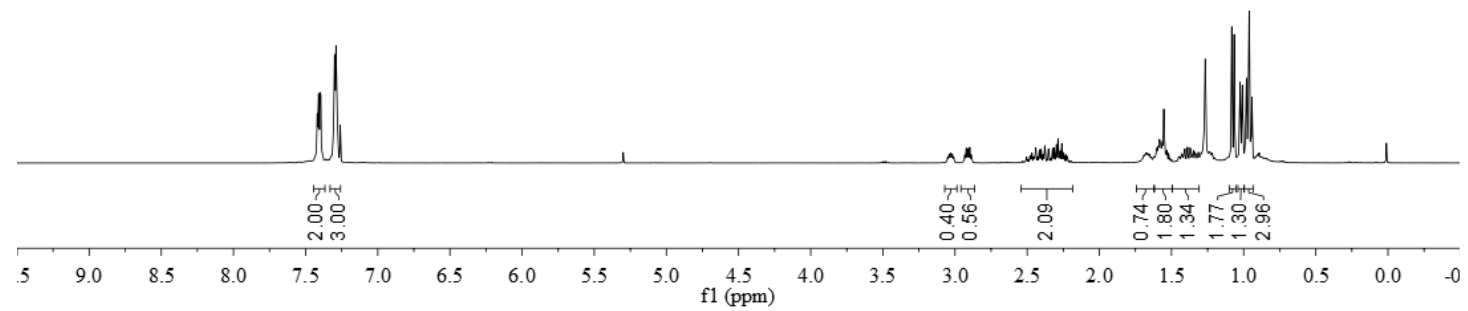




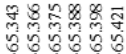 \\ in}<smiles>CCC(C)C(C#CP)CC(F)(F)F</smiles>

$3 \mathrm{ag}\left(\mathrm{CDCl}_{3}, 470 \mathrm{MHz}\right)$

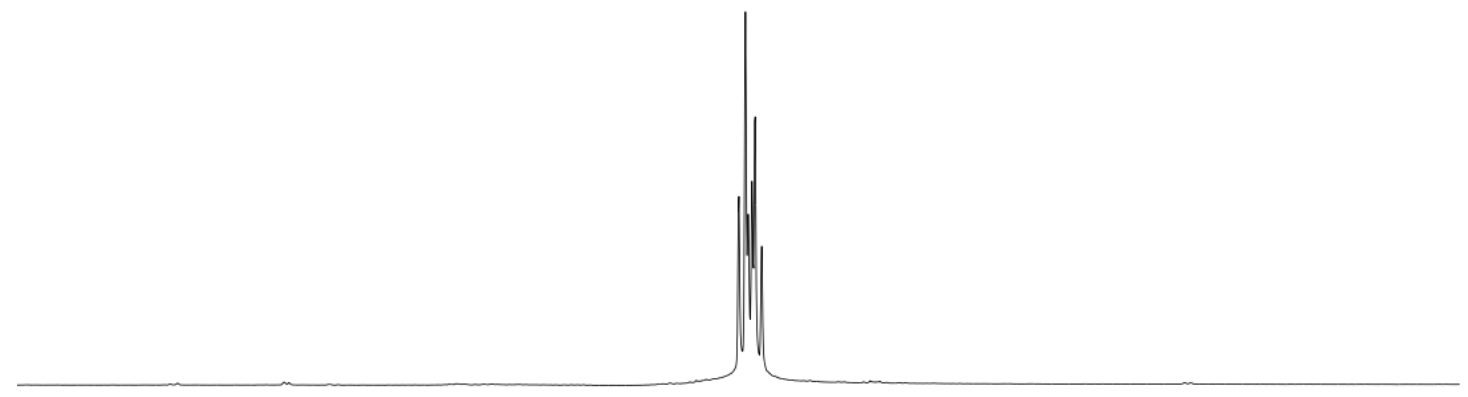

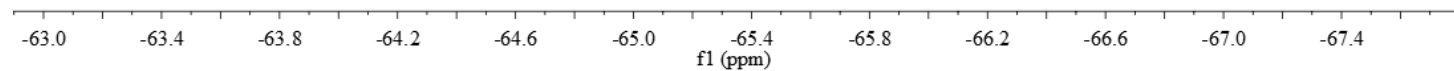

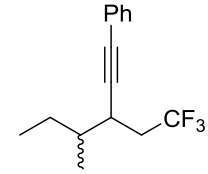

3ag $\left(\mathrm{CDCl}_{3}, 101 \mathrm{MHz}\right)$
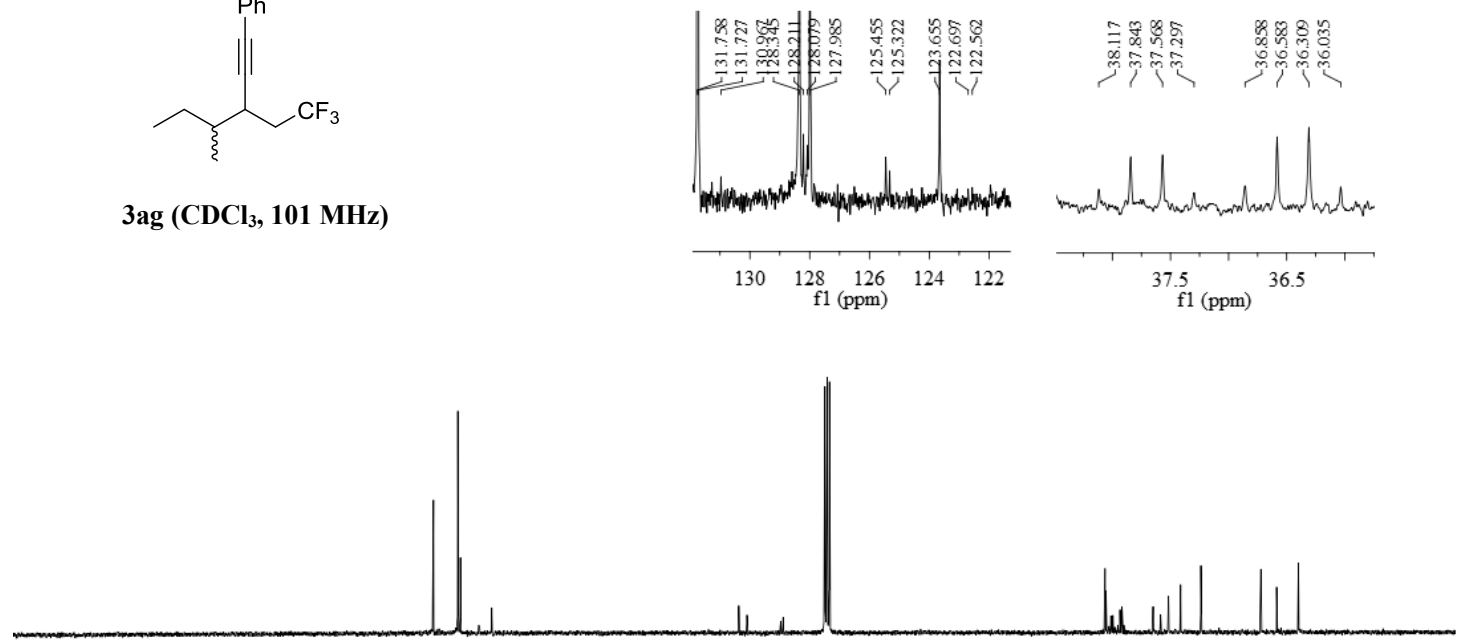

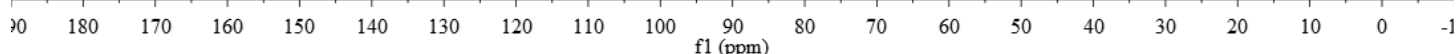




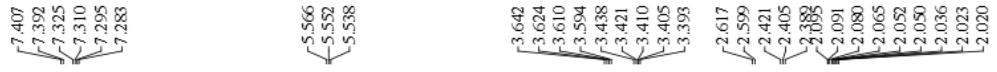<smiles></smiles>

3ah $\left(\mathrm{CDCl}_{3}, 500 \mathrm{MHz}\right)$
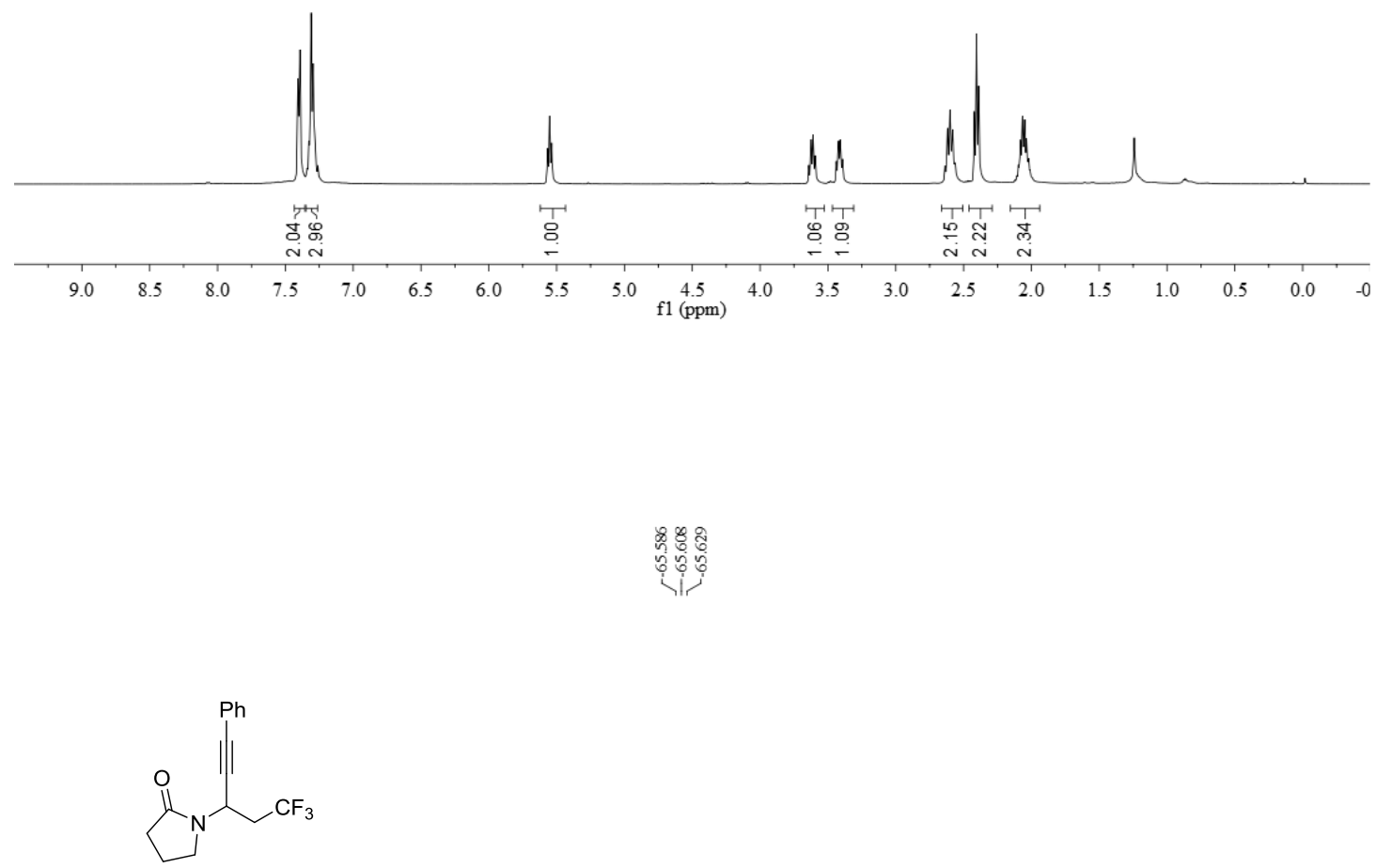

3ah $\left(\mathrm{CDCl}_{3}, 470 \mathrm{MHz}\right)$

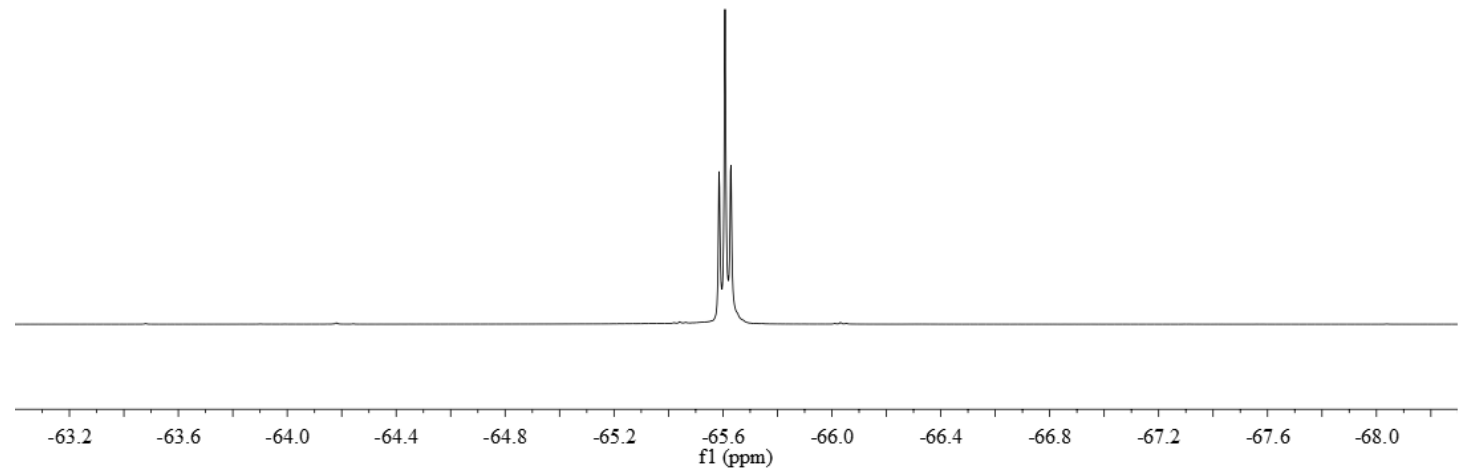


$\underset{\substack{1 \\ \hdashline}}{=}$

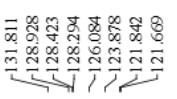

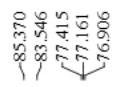

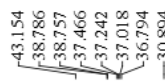

$\stackrel{\sim}{\infty}$

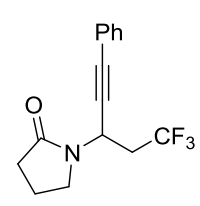

3ah $\left(\mathrm{CDCl}_{3}, 126 \mathrm{MHz}\right)$
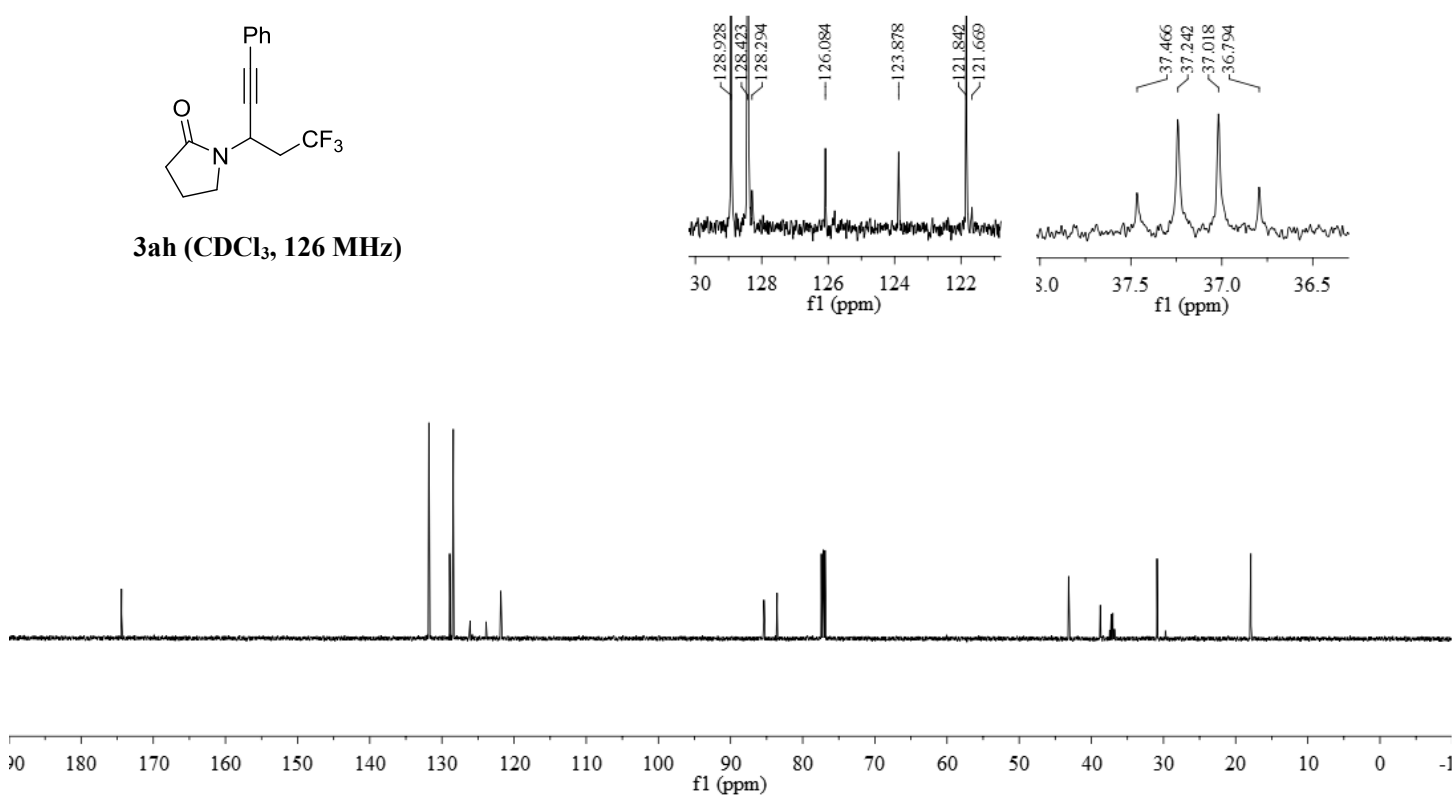

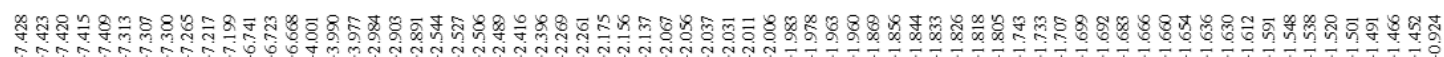

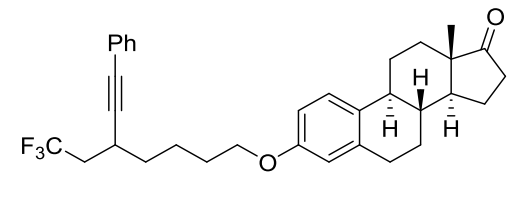

3ai $\left(\mathrm{CDCl}_{3}, 500 \mathrm{MHz}\right)$

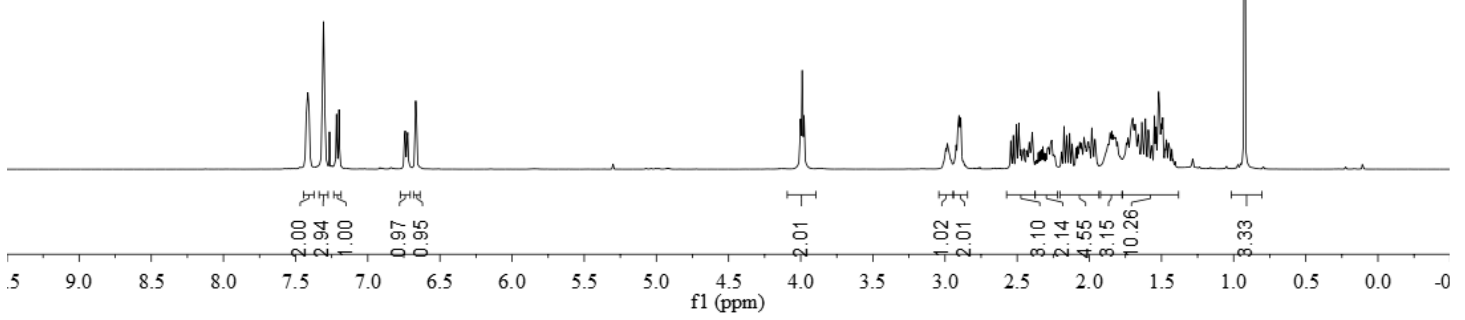

S88 


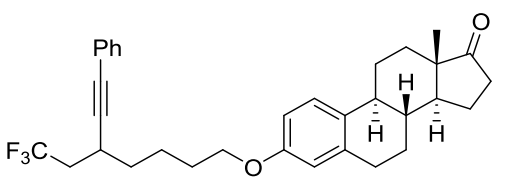

3ai $\left(\mathrm{CDCl}_{3}, 470 \mathrm{MHz}\right)$

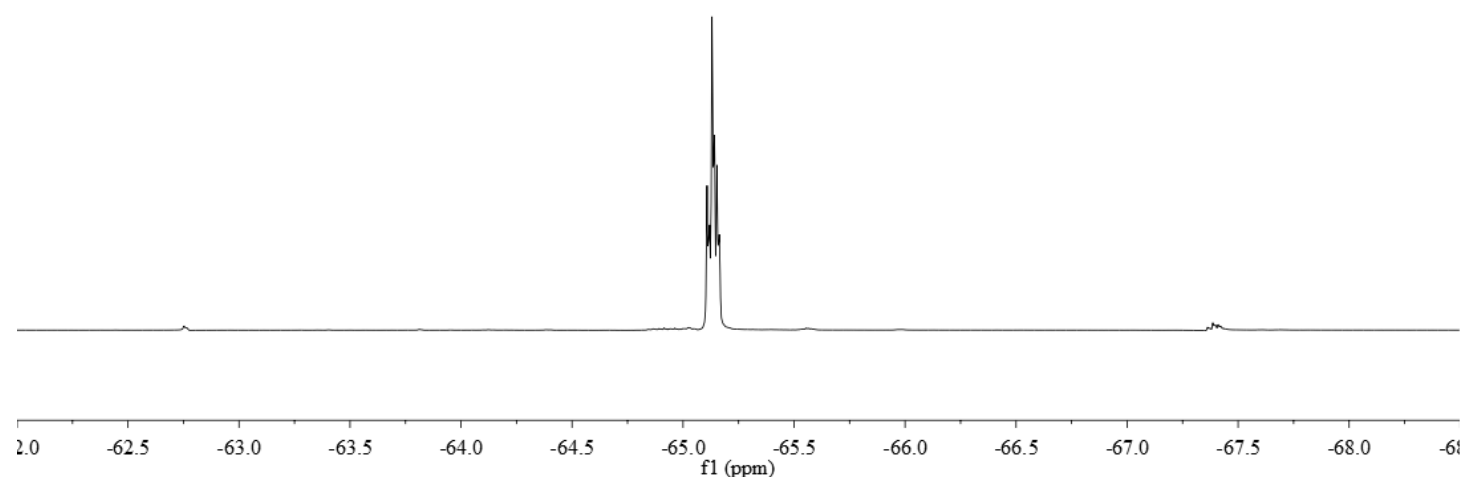<smiles>CC12CCC3c4ccc(OCCCCC(C#CPc5ccccc5)CC(F)(F)F)cc4CCC3C1CCC2=O</smiles>

3ai $\left(\mathrm{CDCl}_{3}, 101 \mathrm{MHz}\right)$
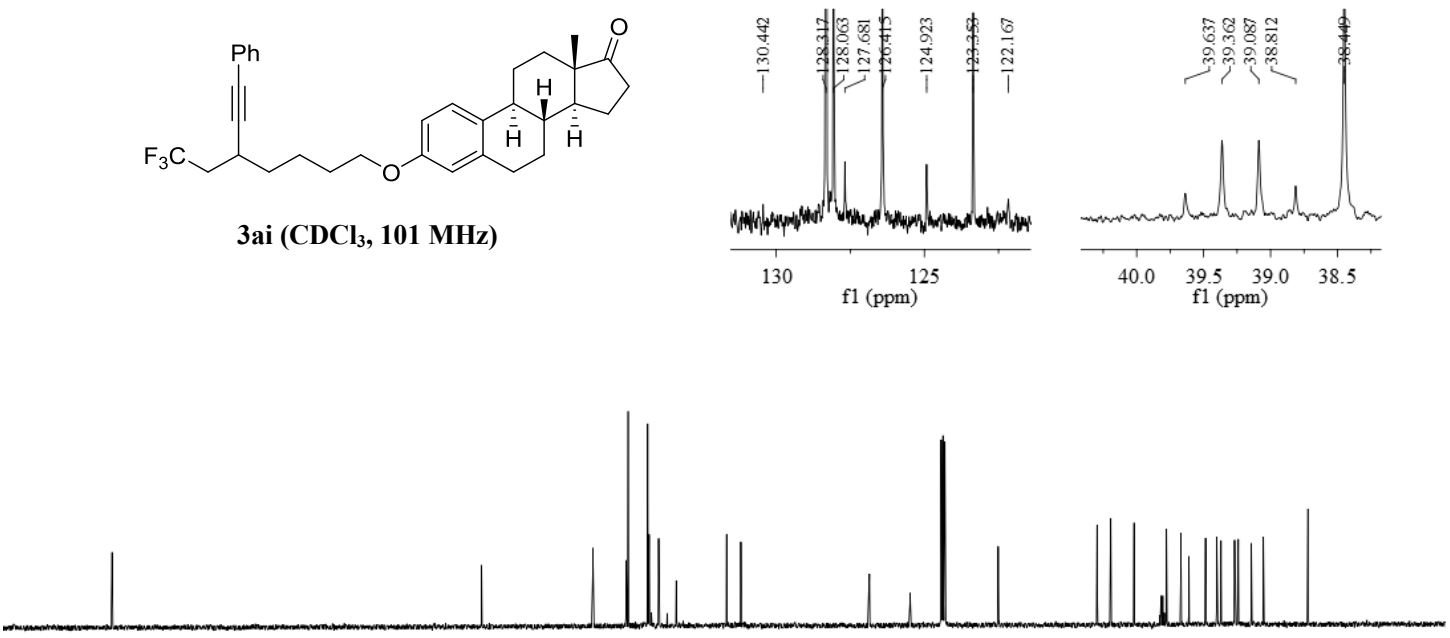

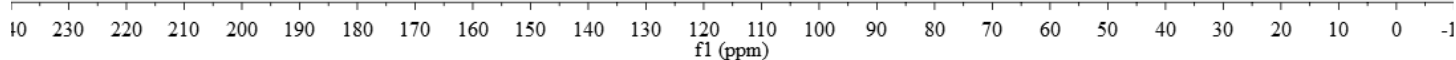




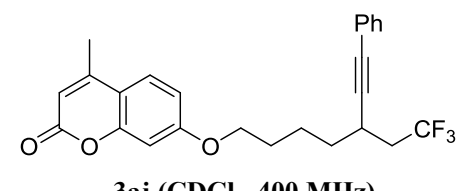

3aj (CDCl 3 , $400 \mathrm{MHz})$

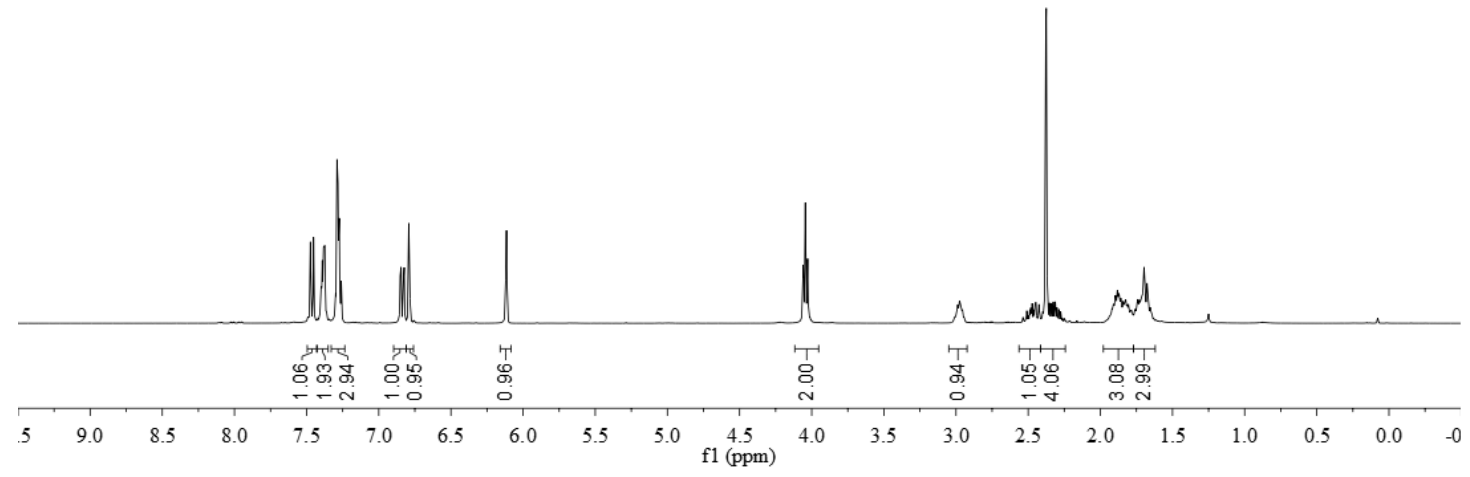

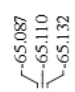

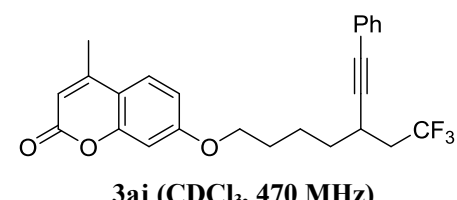

3aj ( $\left.\mathrm{CDCl}_{3}, 470 \mathrm{MHz}\right)$

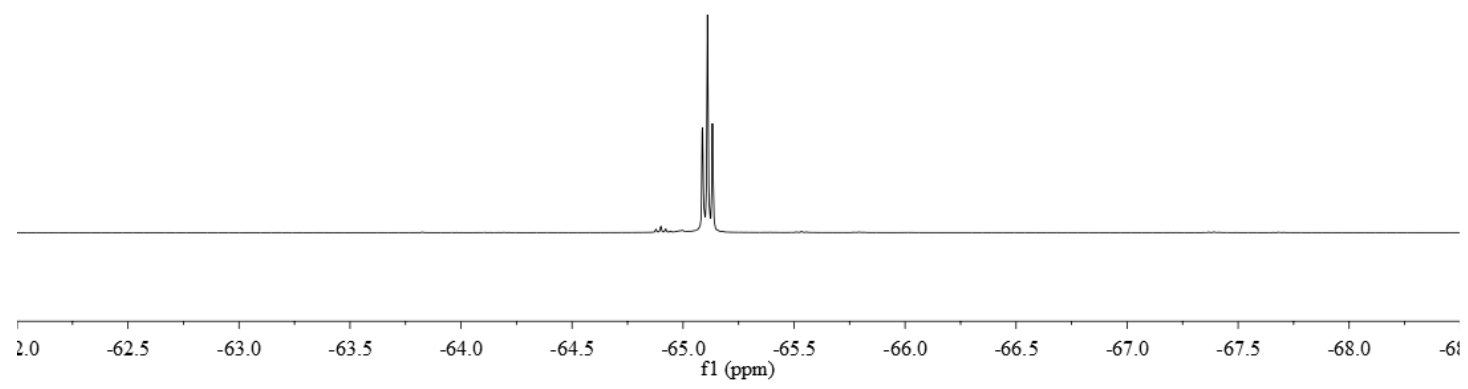




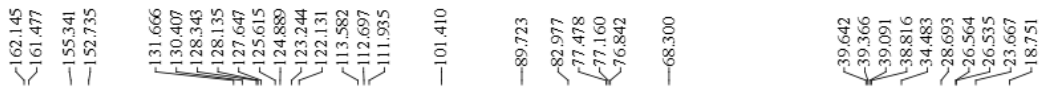<smiles>Cc1cc(=O)oc2cc(OCCCCC(C#Cc3ccccc3)CC(F)(F)F)ccc12</smiles>

3aj $\left(\mathrm{CDCl}_{3}, 101 \mathrm{MHz}\right)$
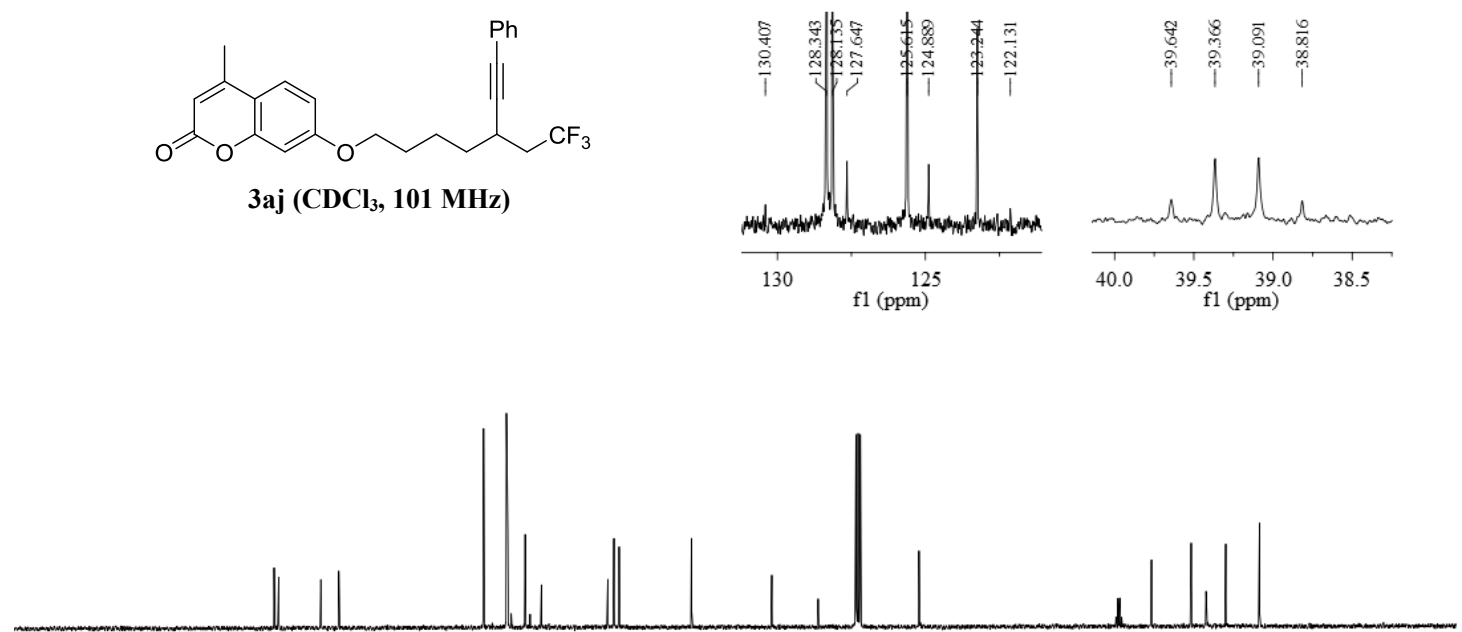

$\begin{array}{llllllllllllllllllllll}10 & 190 & 180 & 170 & 160 & 150 & 140 & 130 & 120 & 110 & \underset{f}{100} \mathbf{f} 1(\mathrm{ppm}) & 80 & 70 & 60 & 50 & 40 & 30 & 20 & 10 & 0 & -1\end{array}$

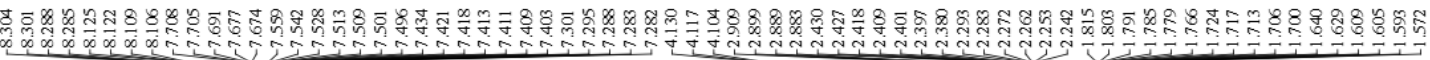

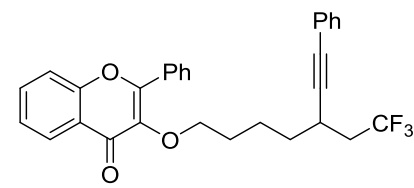

3ak $\left(\mathrm{CDCl}_{3}, 500 \mathrm{MHz}\right)$

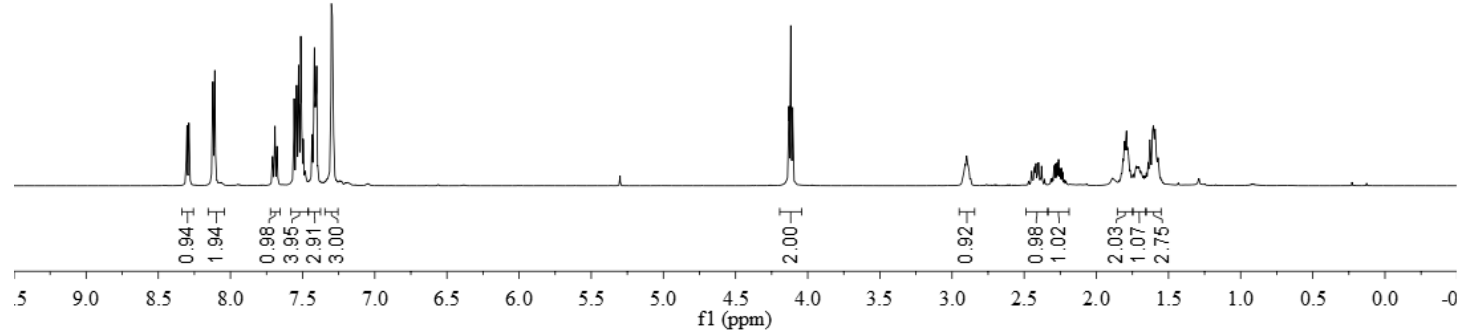




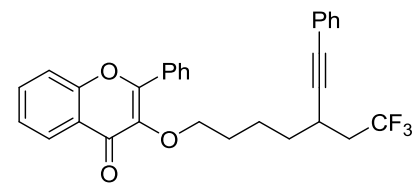

$3 \mathrm{ak}\left(\mathrm{CDCl}_{3}, 470 \mathrm{MHz}\right)$

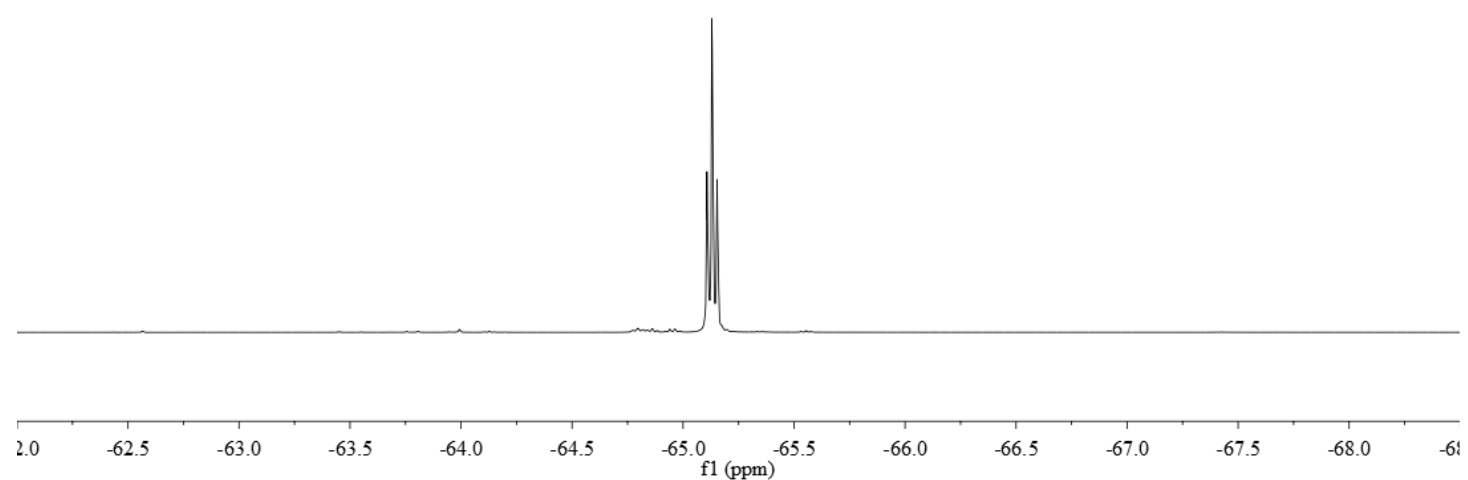

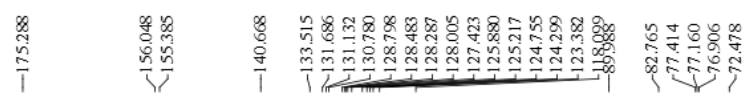

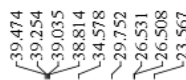

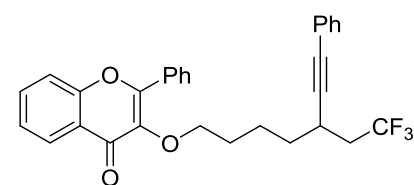

3ak (CDCl, $126 \mathrm{MHz})$
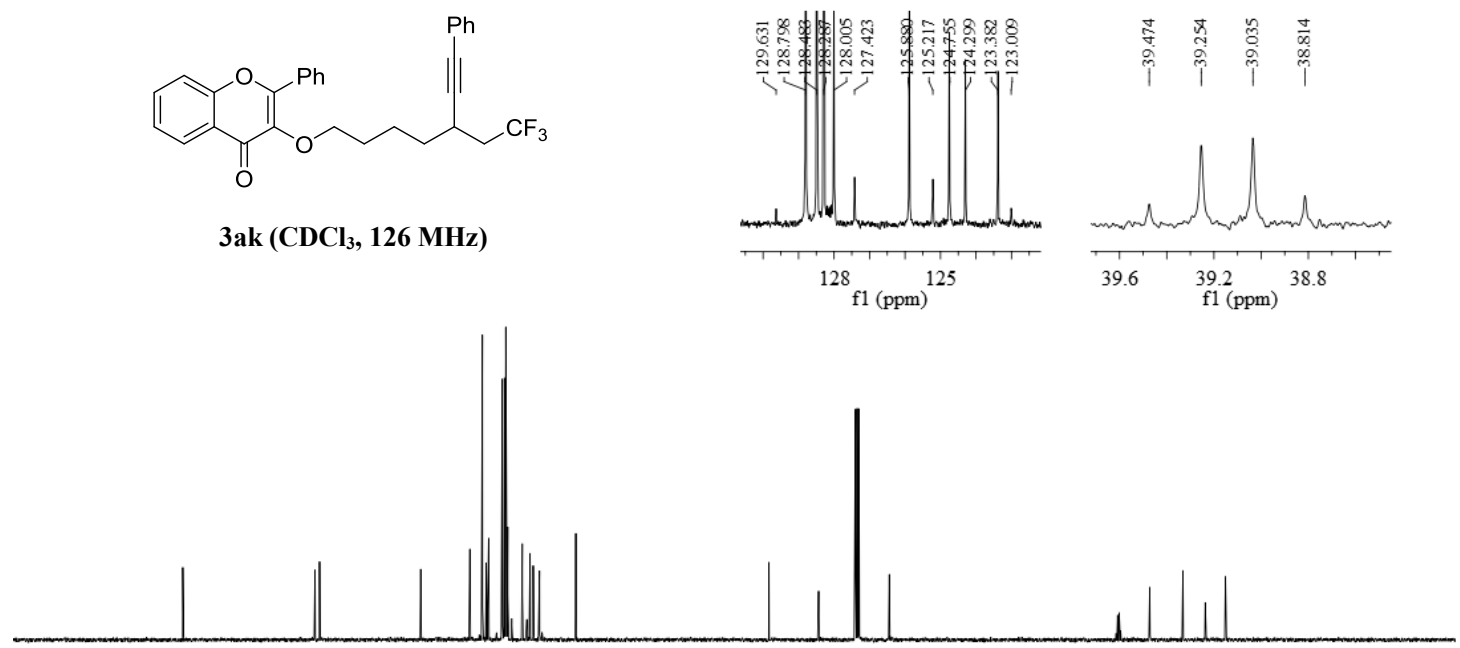

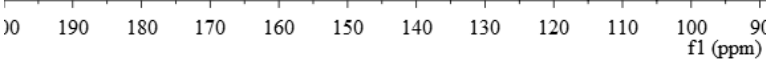




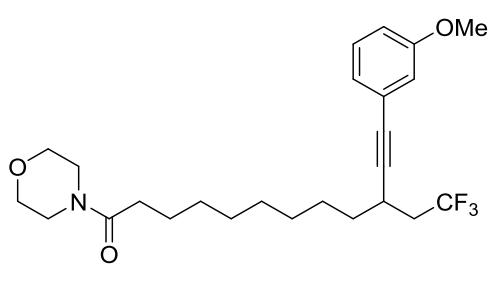

3al $\left(\mathrm{CDCl}_{3}, 400 \mathrm{MHz}\right)$

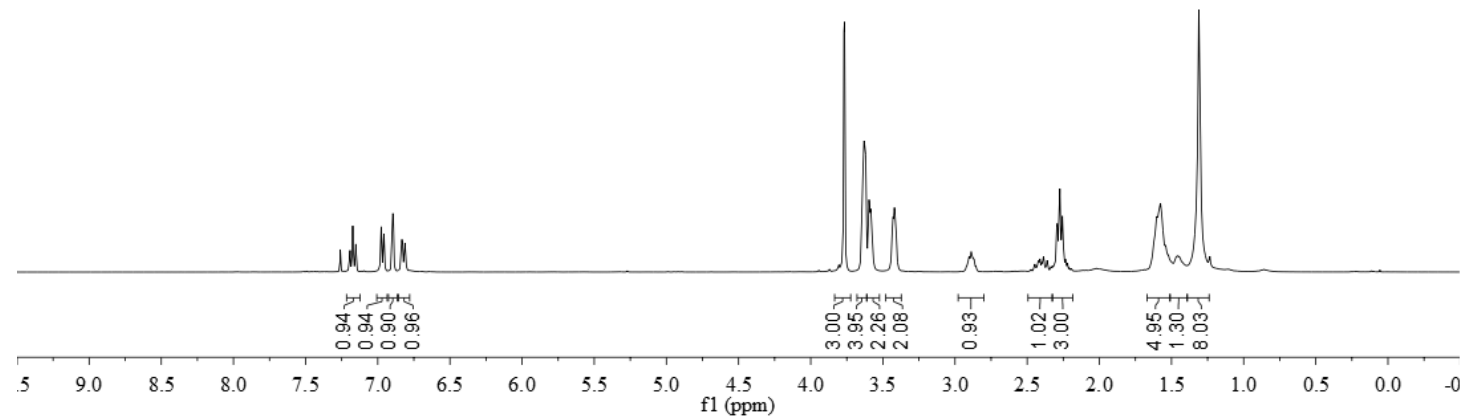

嫼楬

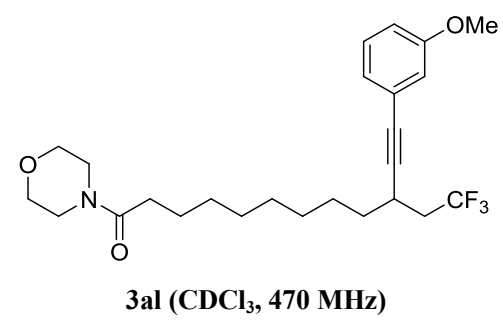

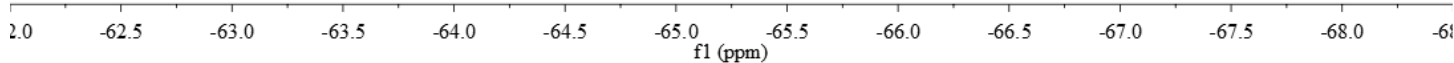




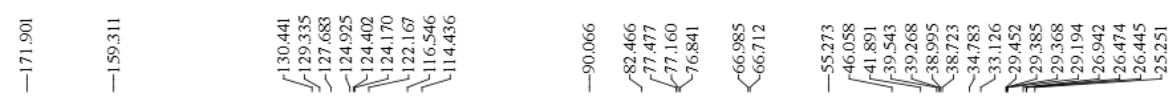

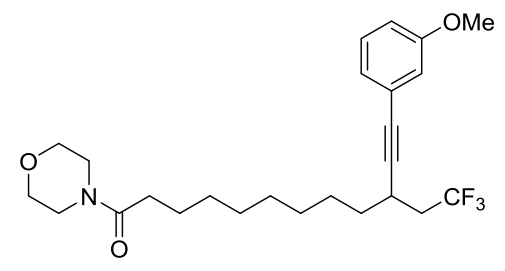

3al $\left(\mathrm{CDCl}_{3}, 101 \mathrm{MHz}\right)$
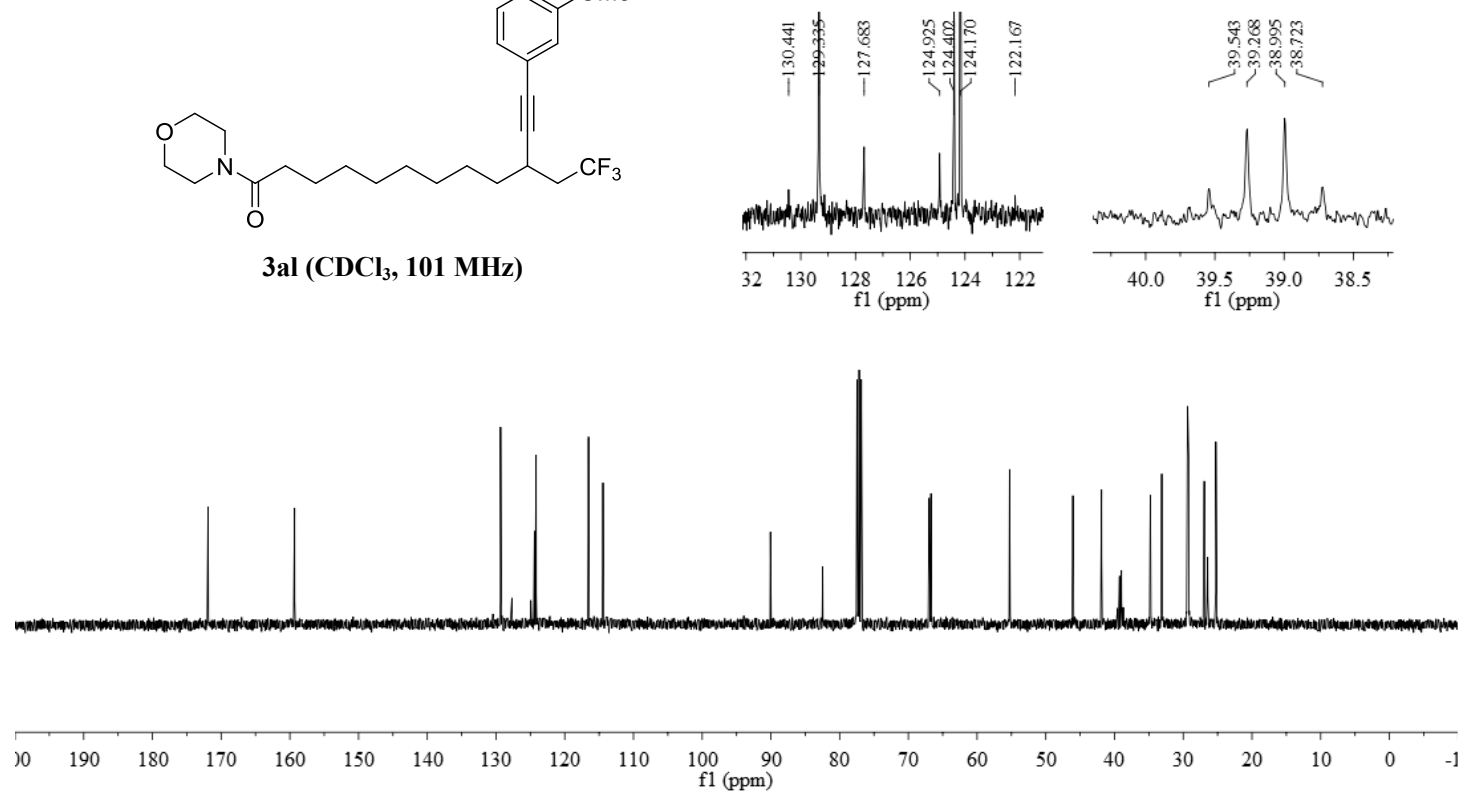

원돈

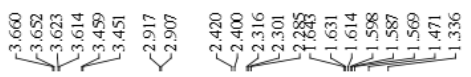

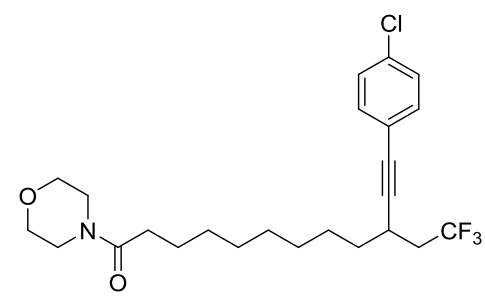

$3 \mathrm{am}\left(\mathrm{CDCl}_{3}, 500 \mathrm{MHz}\right)$

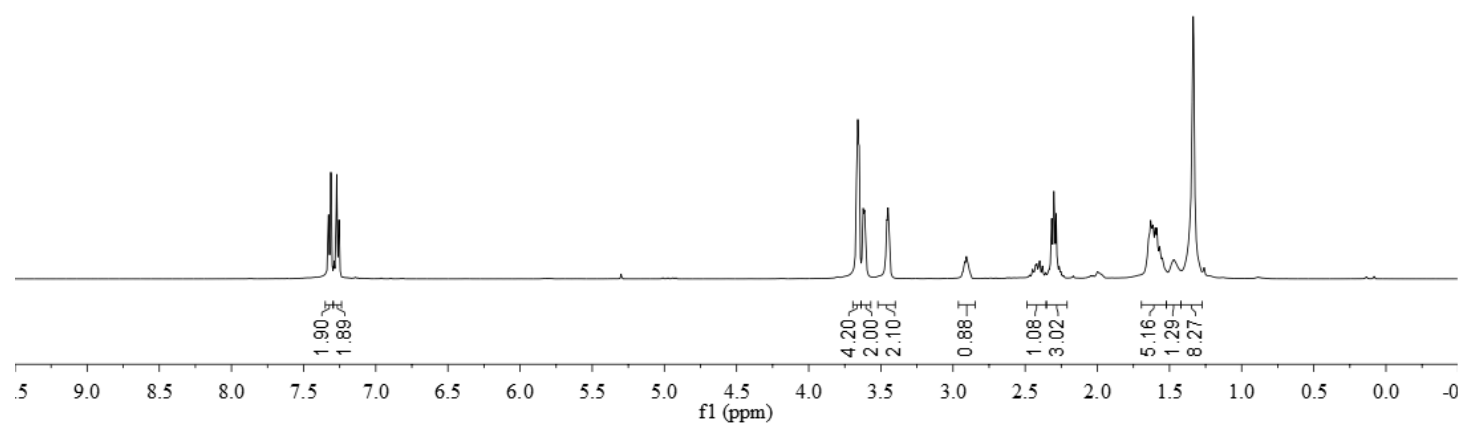




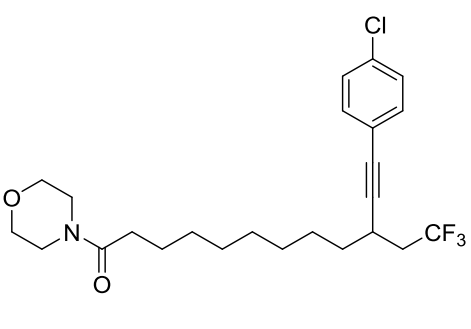

3am (CDCl 3 , $470 \mathrm{MHz})$
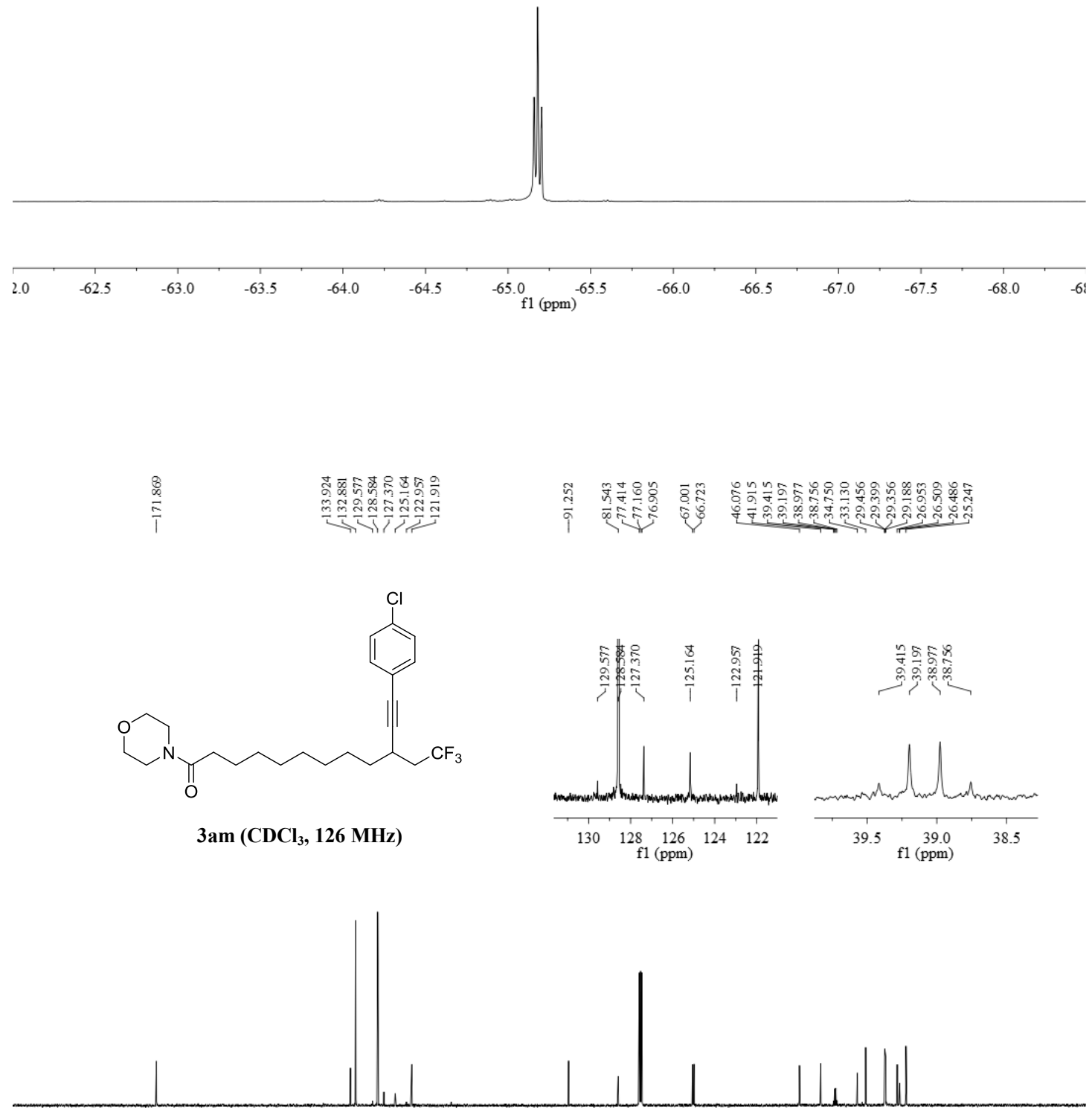

$\begin{array}{lllllllllll}10 & 190 & 180 & 170 & 160 & 150 & 140 & 130 & 120 & 110 & \begin{array}{c}100 \\ \mathrm{f} 1(\mathrm{ppm})\end{array}\end{array}$ 


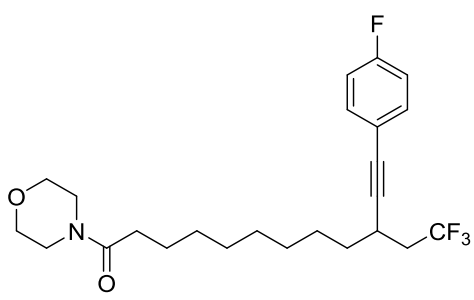

3an $\left(\mathrm{CDCl}_{3}, 400 \mathrm{MHz}\right)$

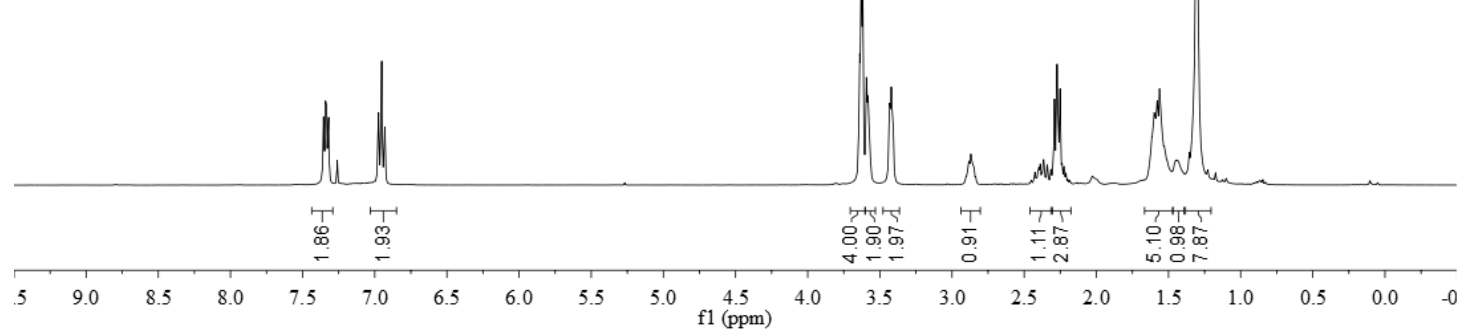

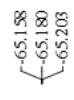

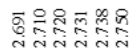
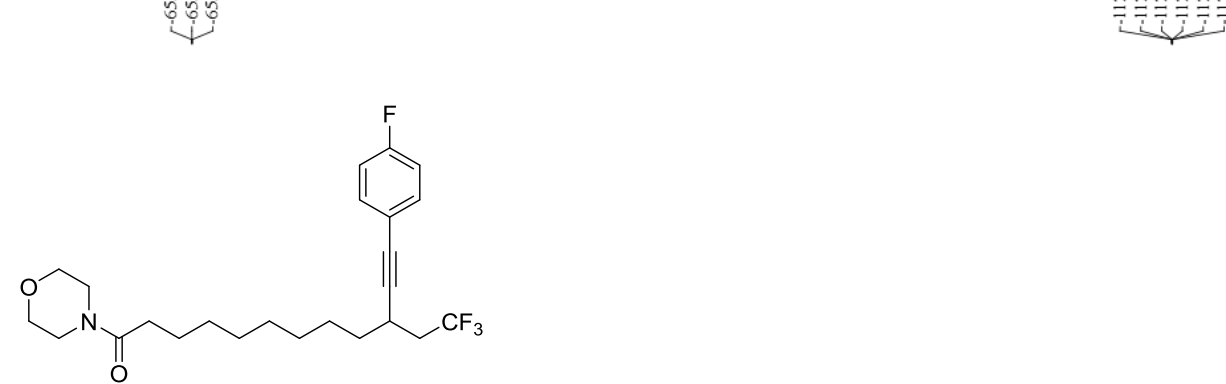

3 an $\left(\mathrm{CDCl}_{3}, 470 \mathrm{MHz}\right)$

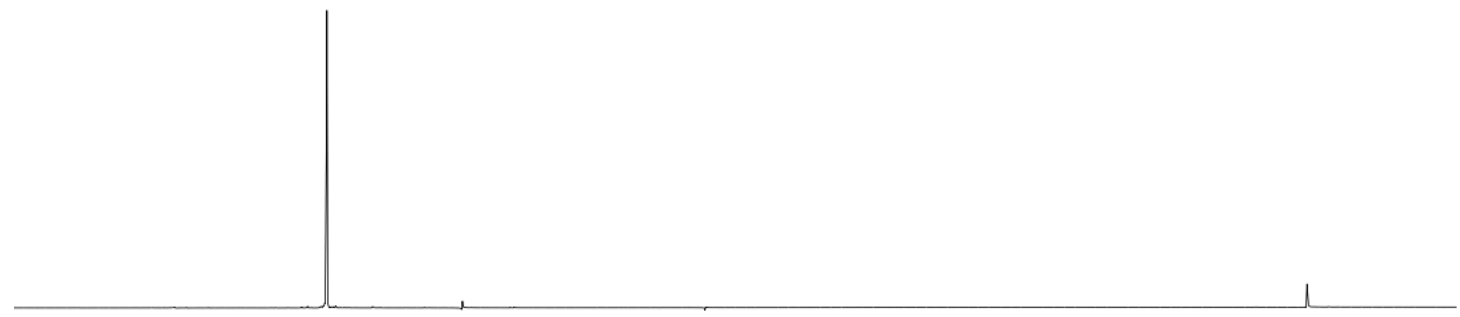

;0 $\quad-55 \quad-60$

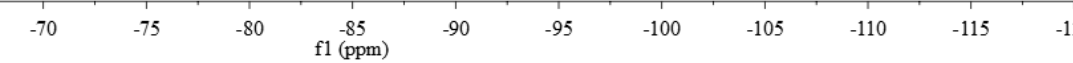



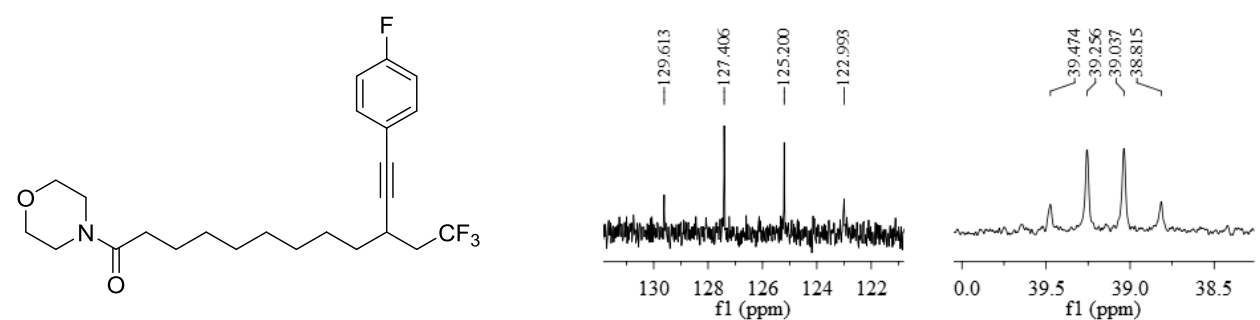

3an $\left(\mathrm{CDCl}_{3}, 126 \mathrm{MHz}\right)$
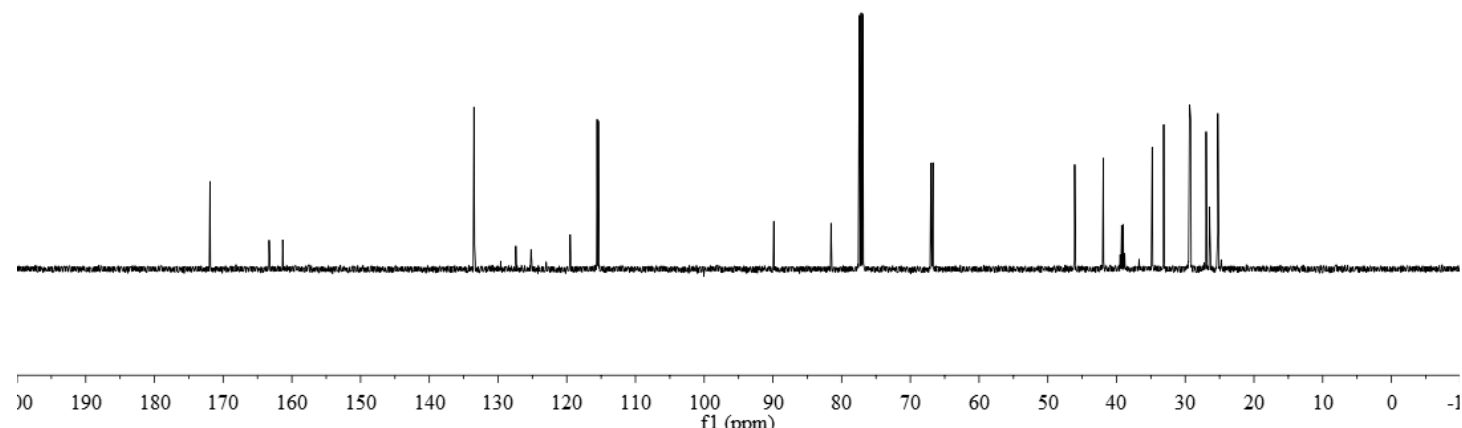

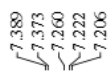

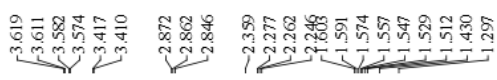
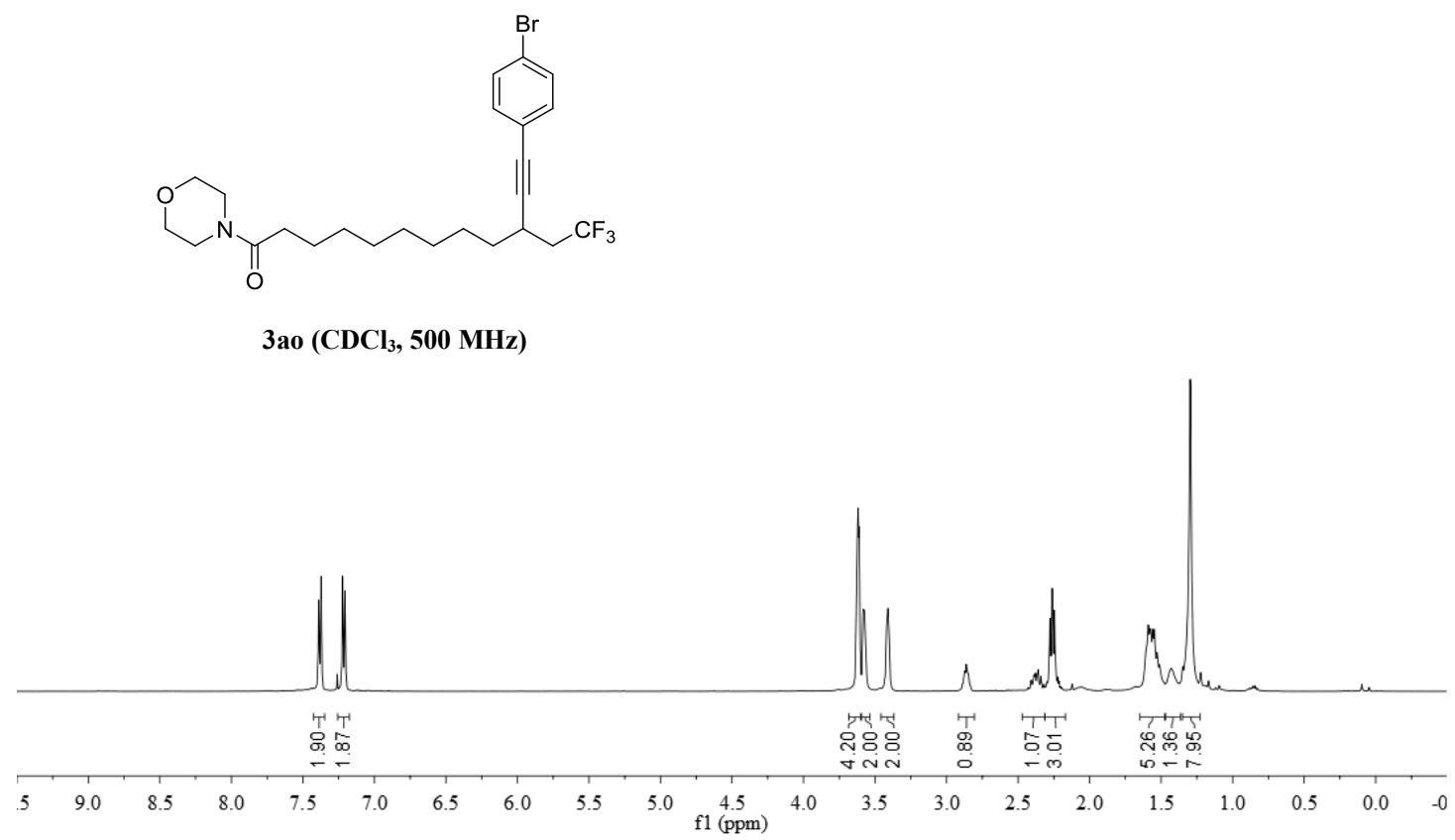


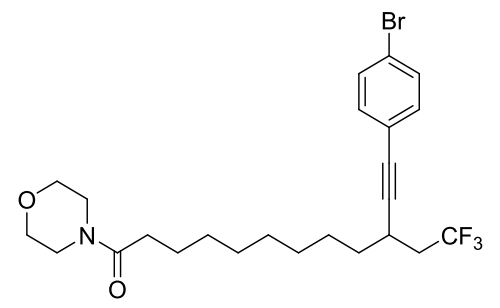

$3 \mathrm{ao}\left(\mathrm{CDCl}_{3}, 470 \mathrm{MHz}\right)$

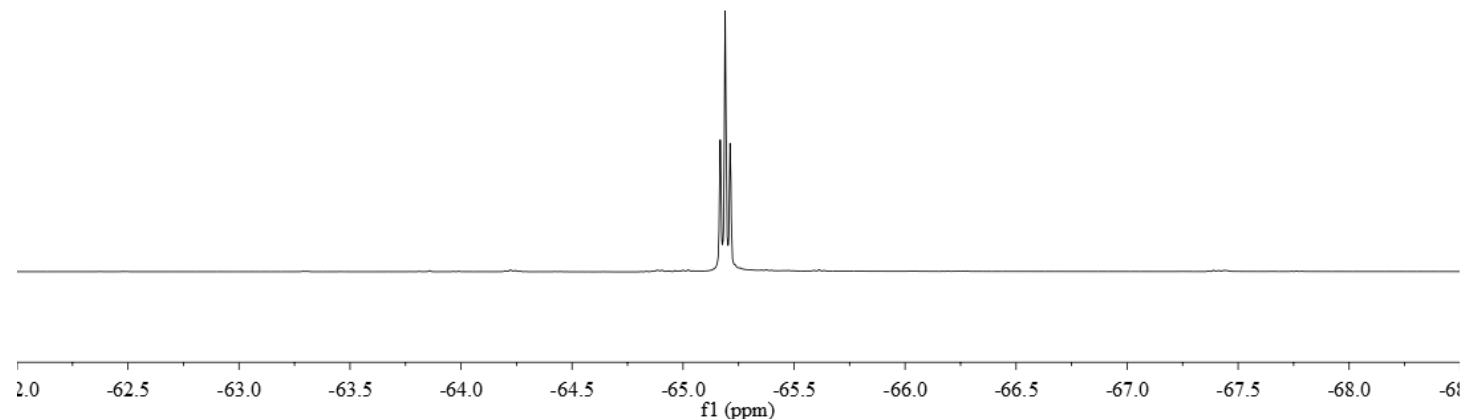

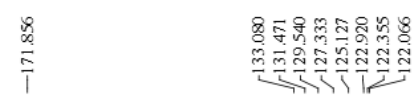
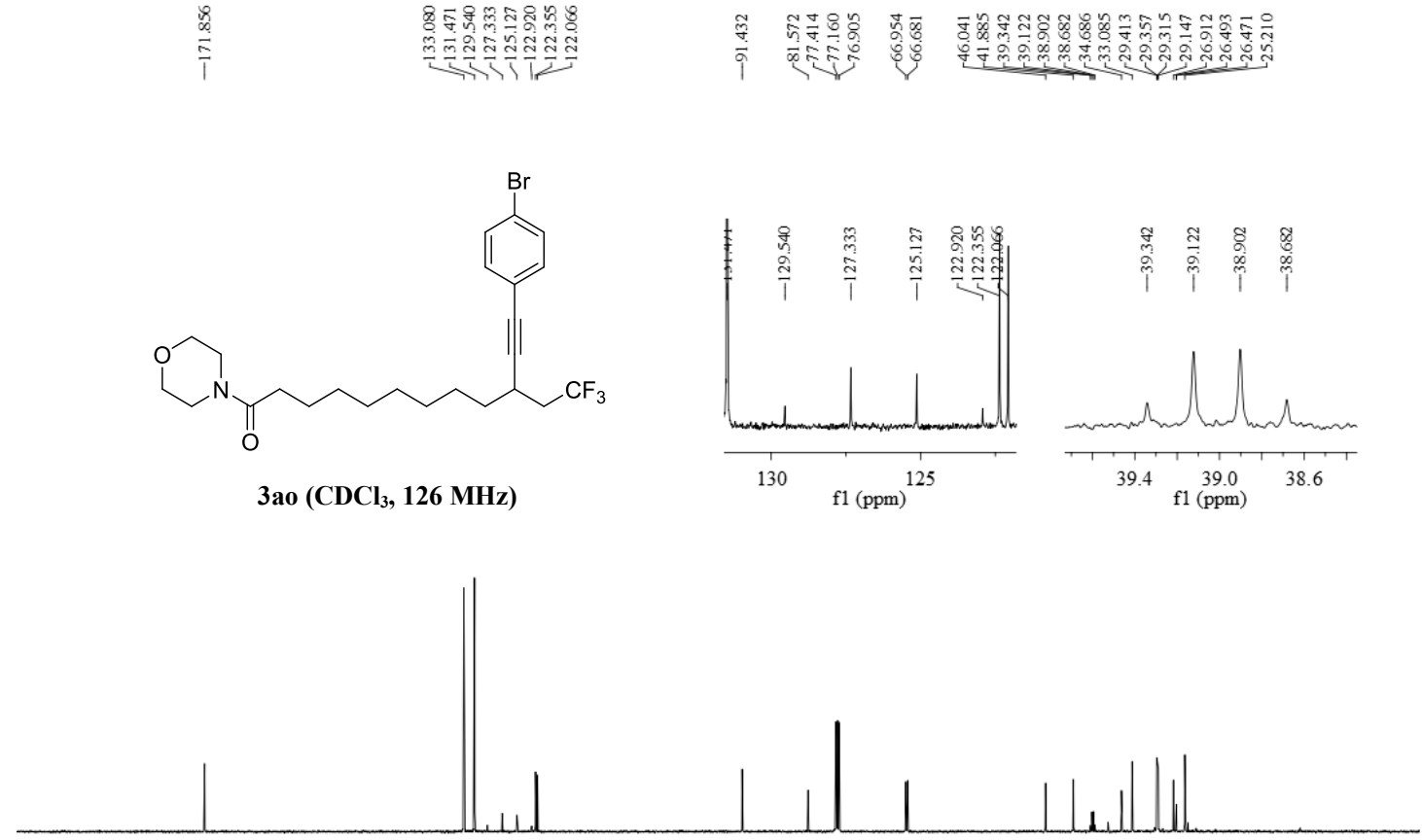

$\begin{array}{lllllllllll}10 & 190 & 180 & 170 & 160 & 150 & 140 & 130 & 120 & 110 & \begin{array}{c}100 \\ \mathrm{f} 1(\mathrm{ppm})\end{array}\end{array}$ 


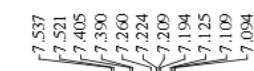

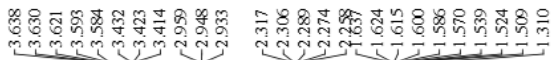

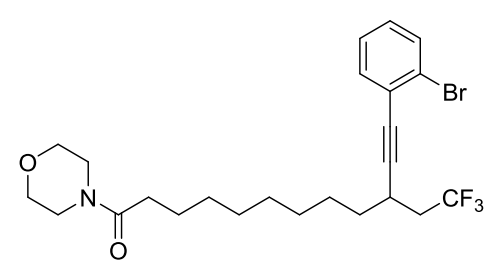

3ap $\left(\mathrm{CDCl}_{3}, 500 \mathrm{MHz}\right)$

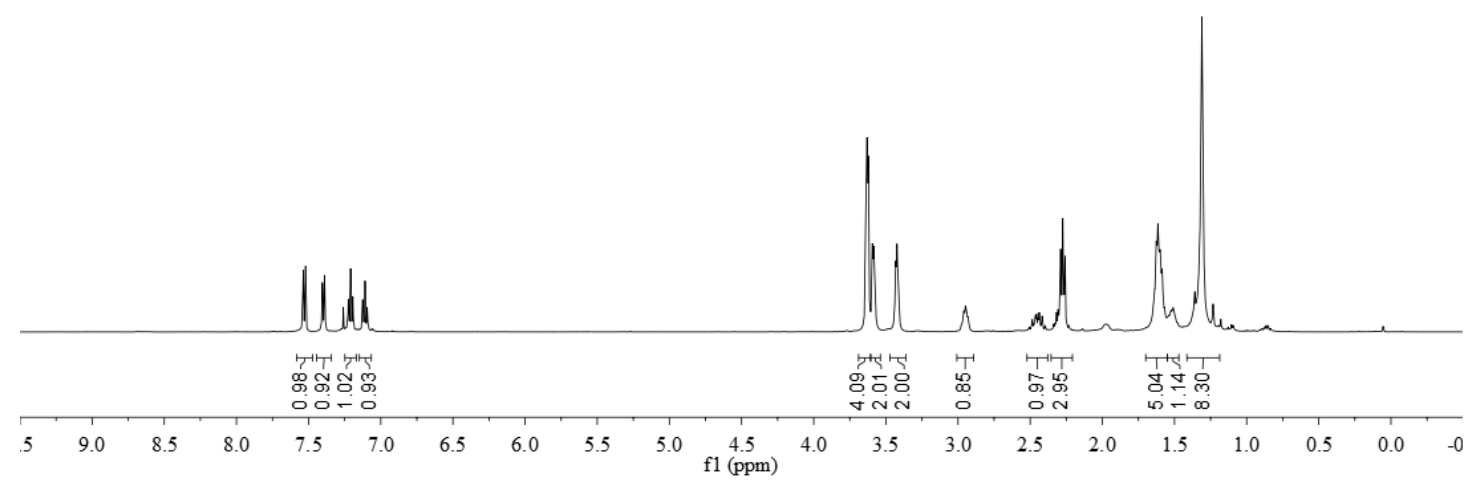

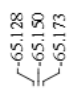

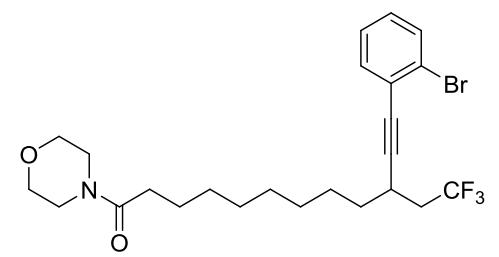

3ap (CDCl, $470 \mathrm{MHz})$

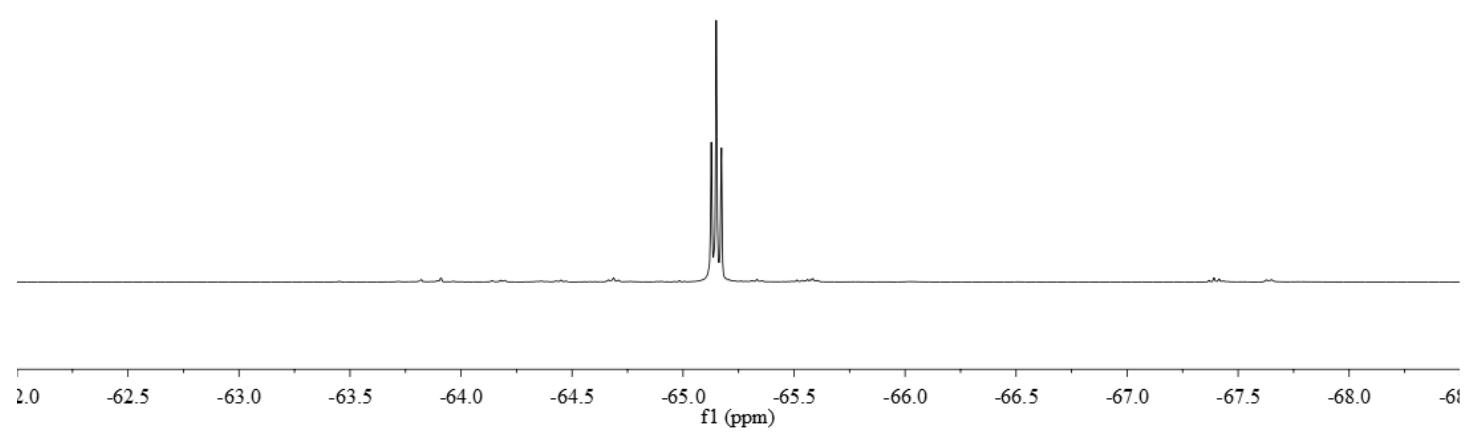



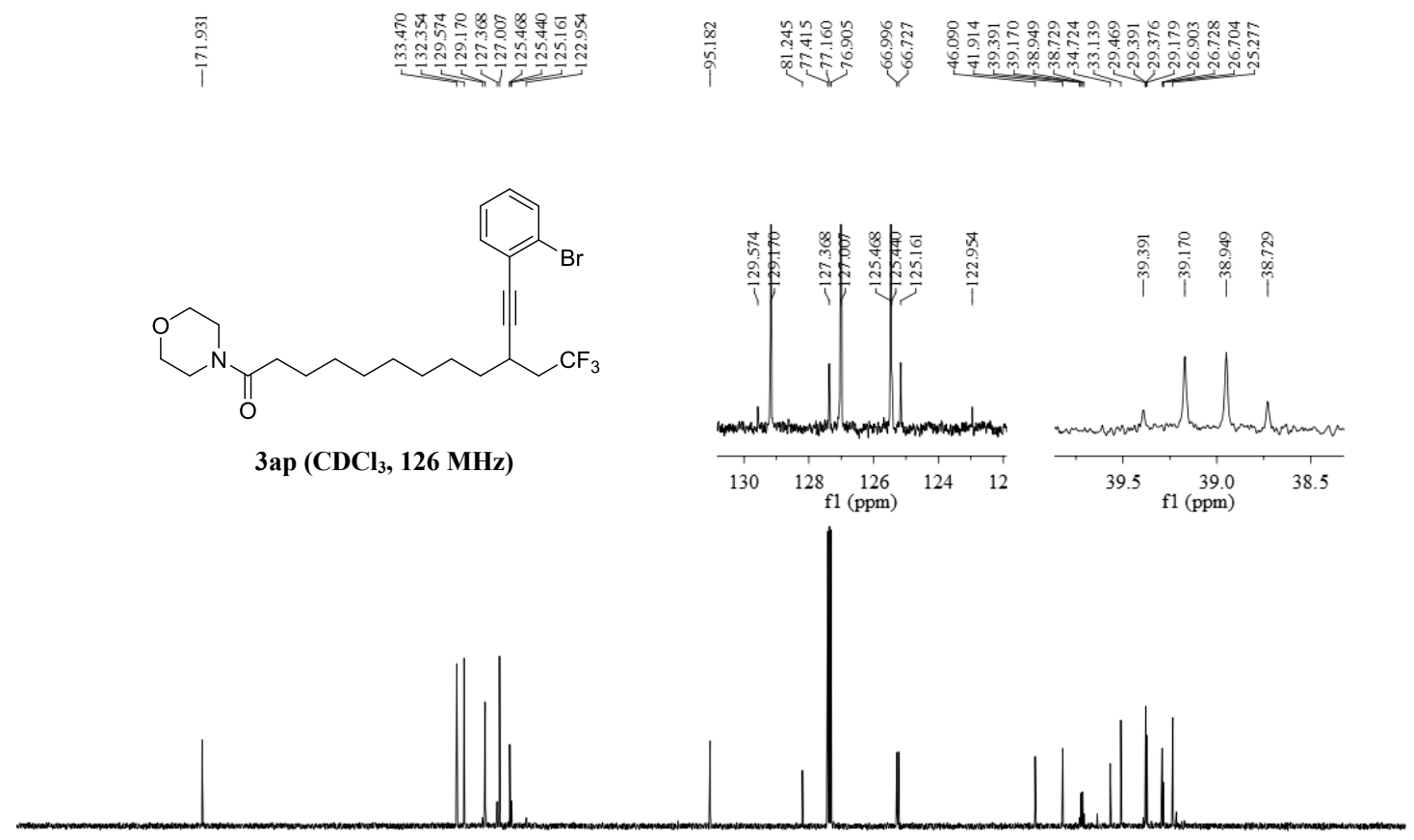

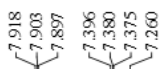

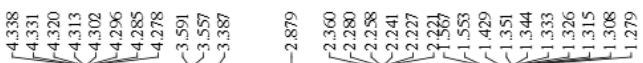
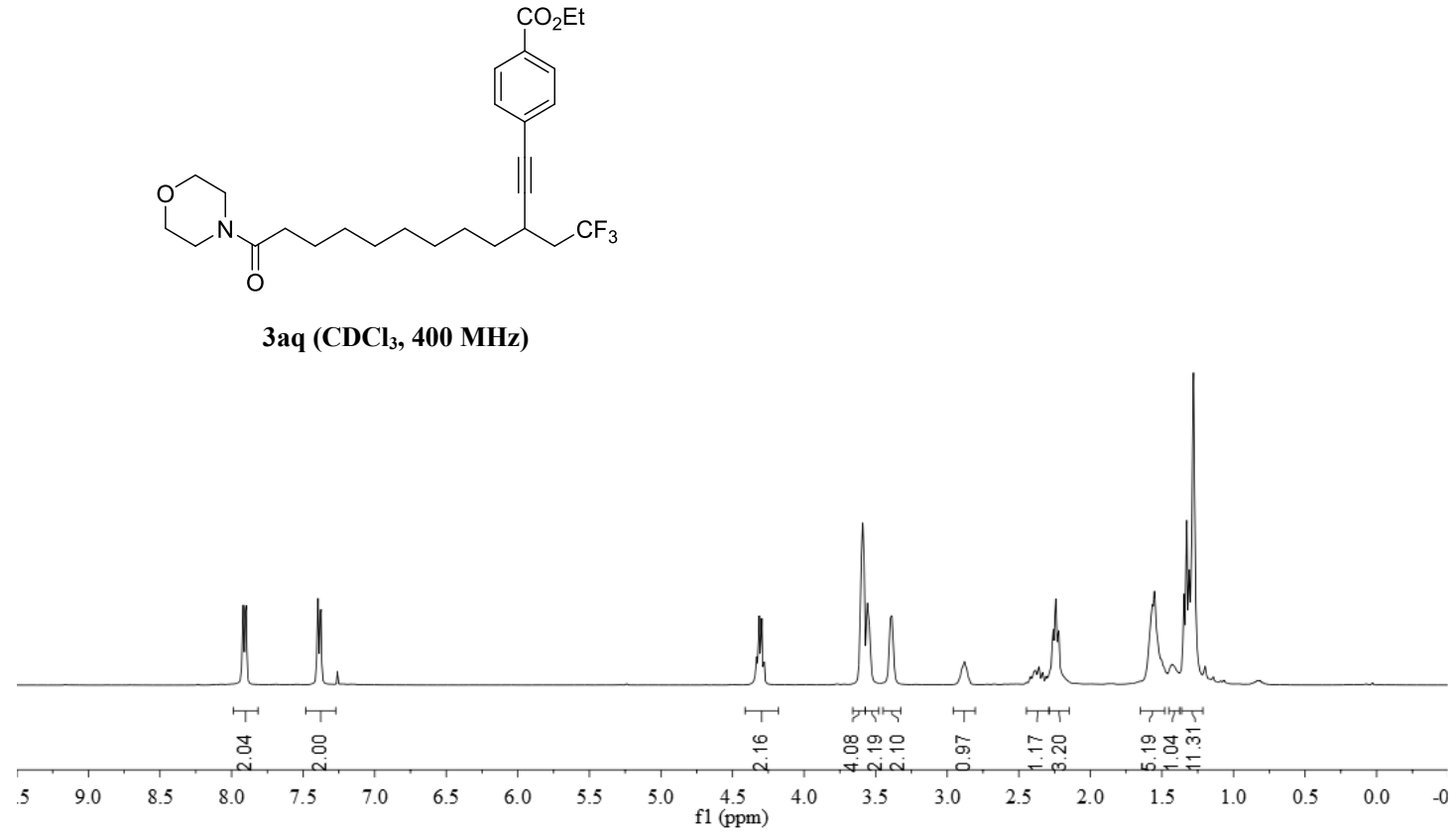


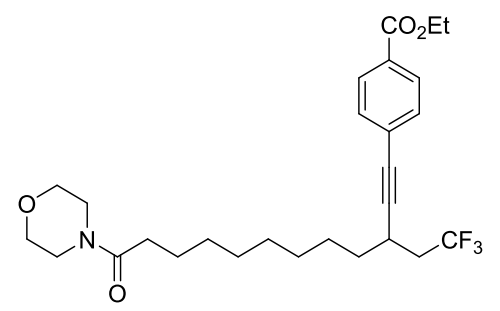

$3 \mathrm{aq}\left(\mathrm{CDCl}_{3}, 470 \mathrm{MHz}\right)$

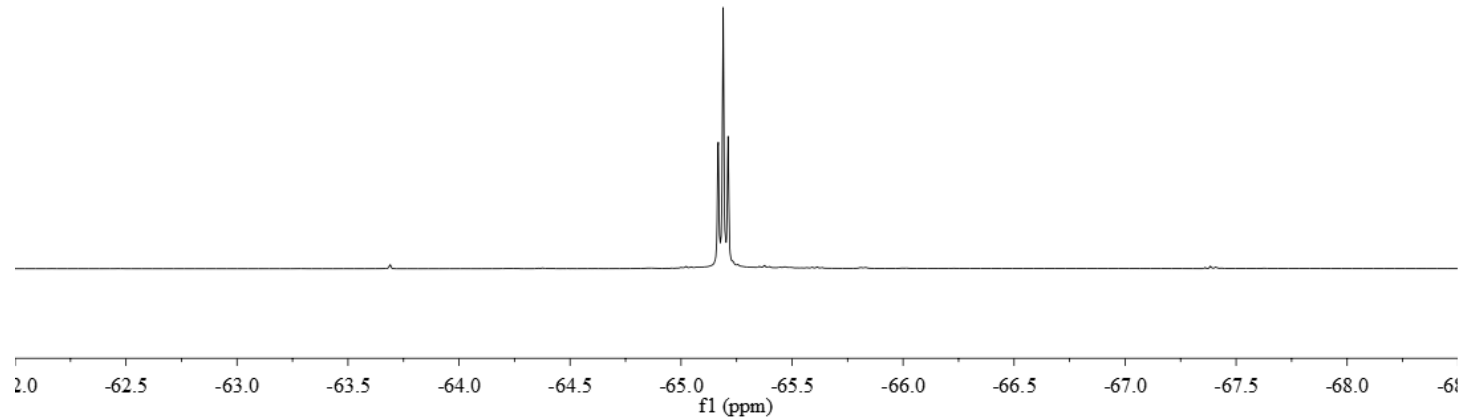

暲

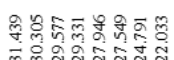

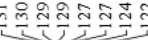

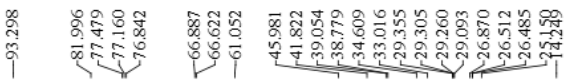

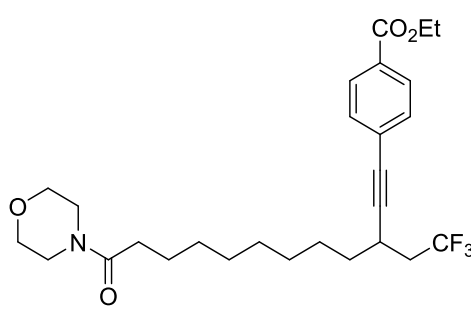

$3 \mathrm{aq}\left(\mathrm{CDCl}_{3}, 101 \mathrm{MHz}\right)$
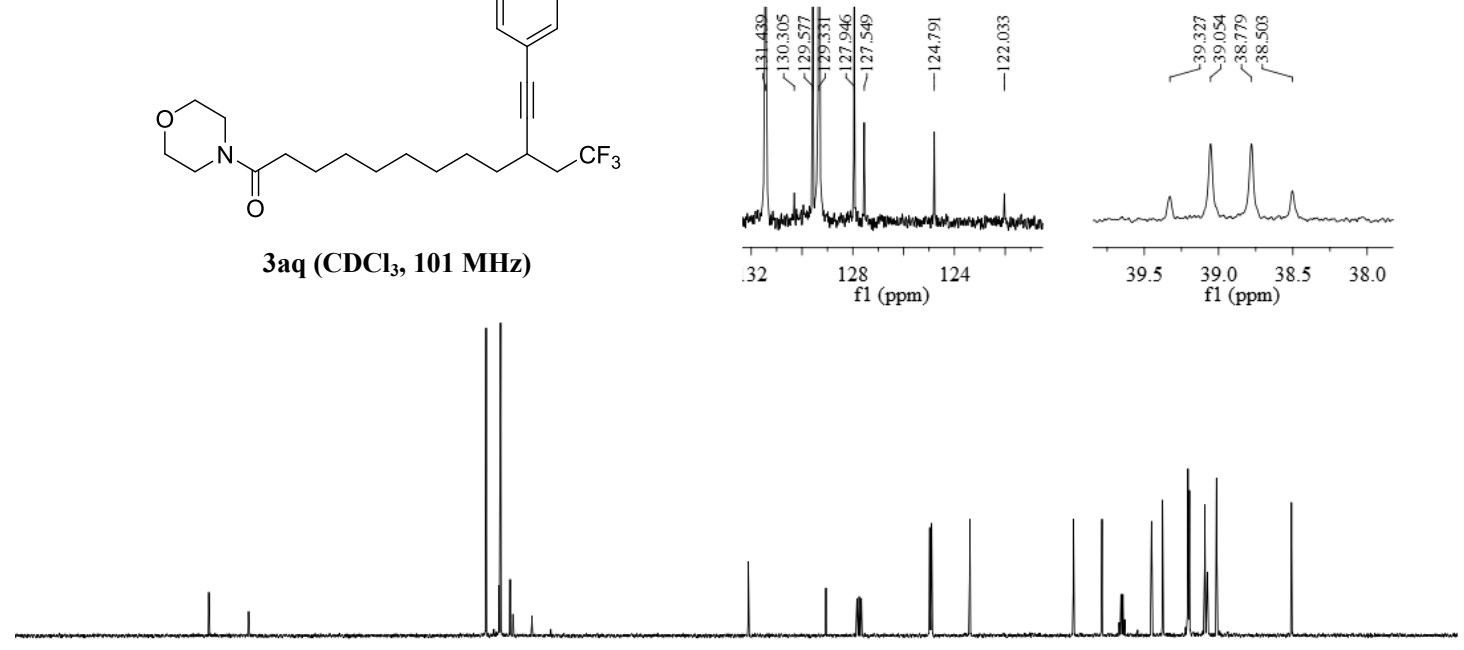

$\begin{array}{lllllllllll}10 & 190 & 180 & 170 & 160 & 150 & 140 & 130 & 120 & 110 & \begin{array}{c}100 \\ \mathrm{f} 1(\mathrm{ppm})\end{array}\end{array}$ 


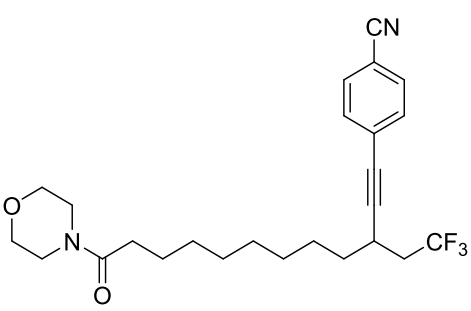

3ar $\left(\mathrm{CDCl}_{3}, 400 \mathrm{MHz}\right)$

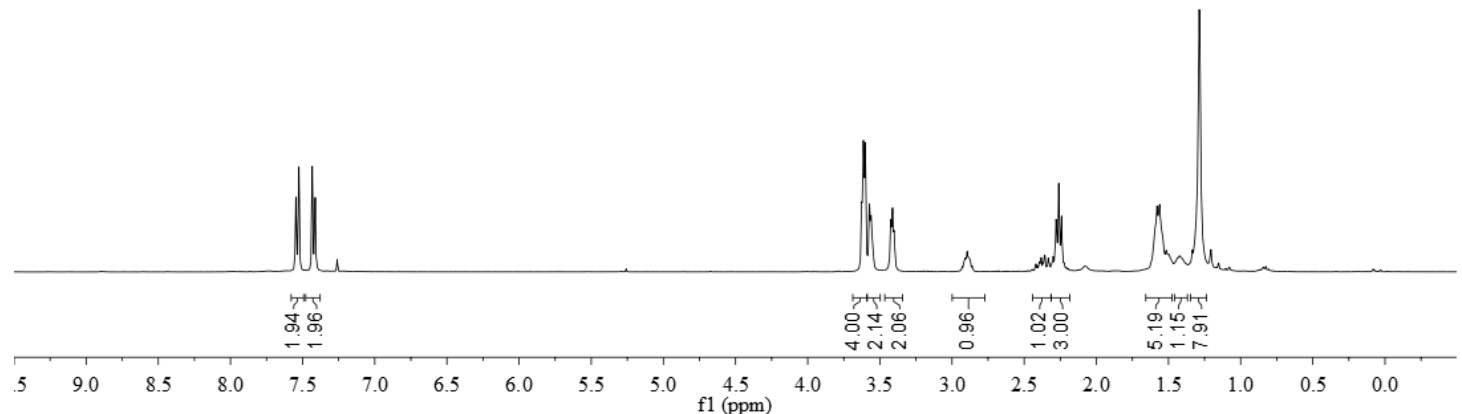

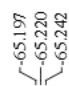

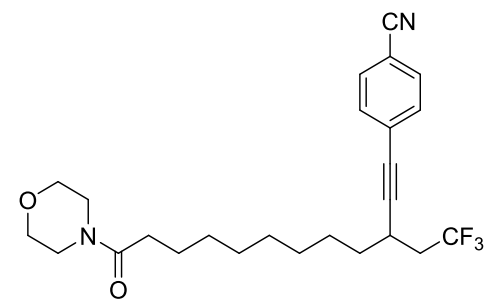

$3 \operatorname{ar}\left(\mathrm{CDCl}_{3}, 470 \mathrm{MHz}\right)$

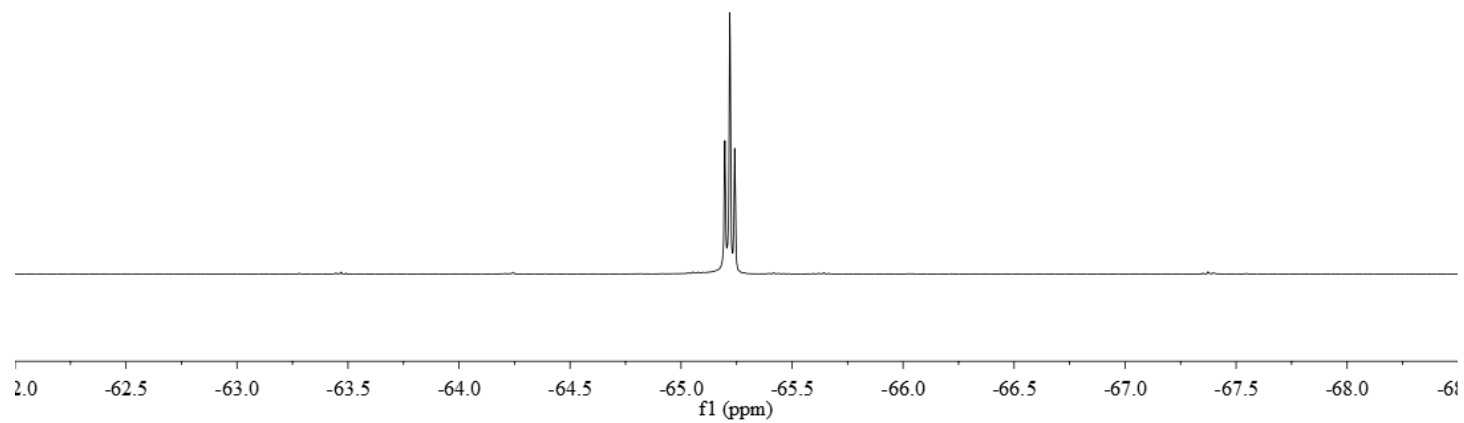



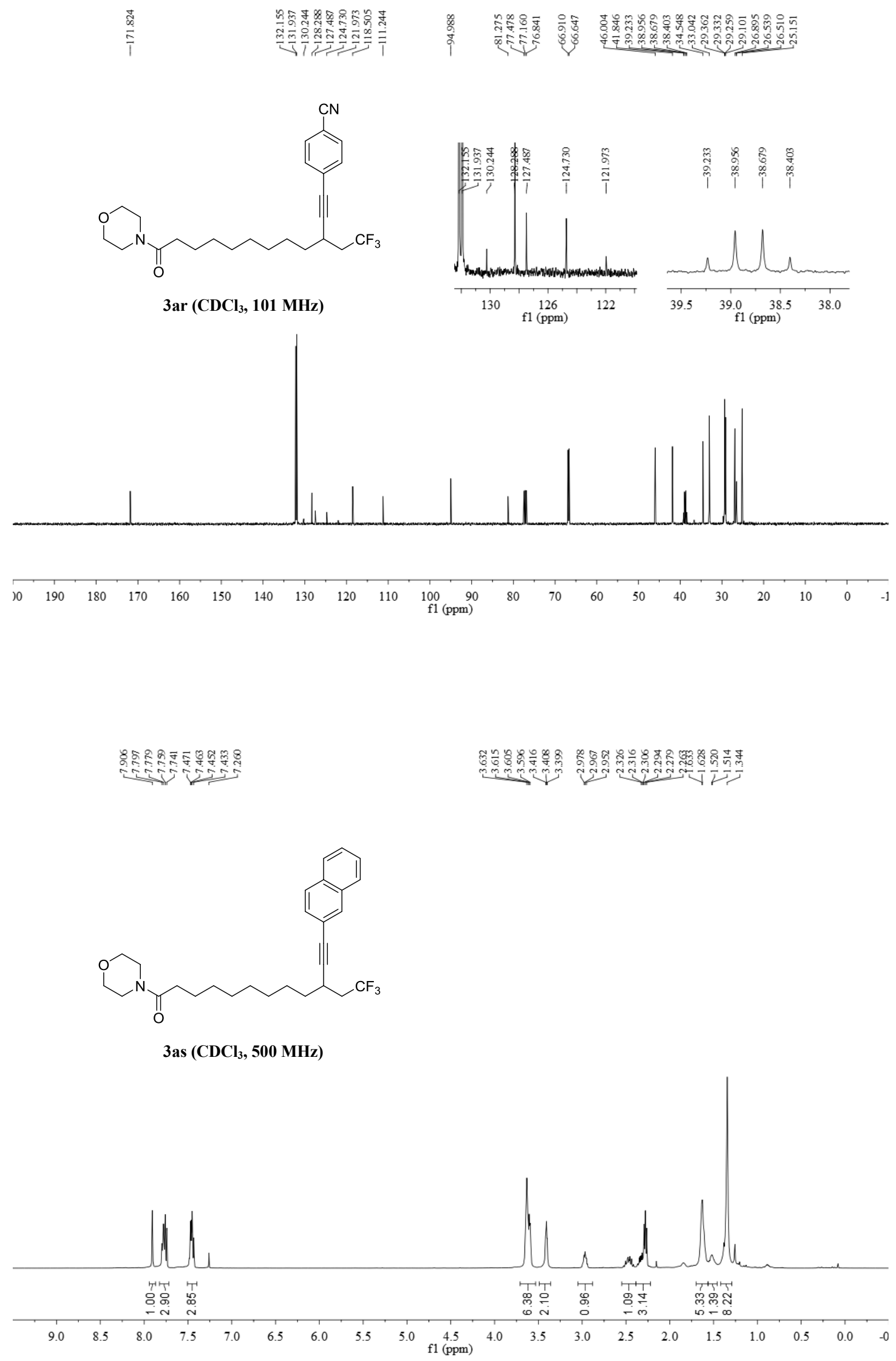


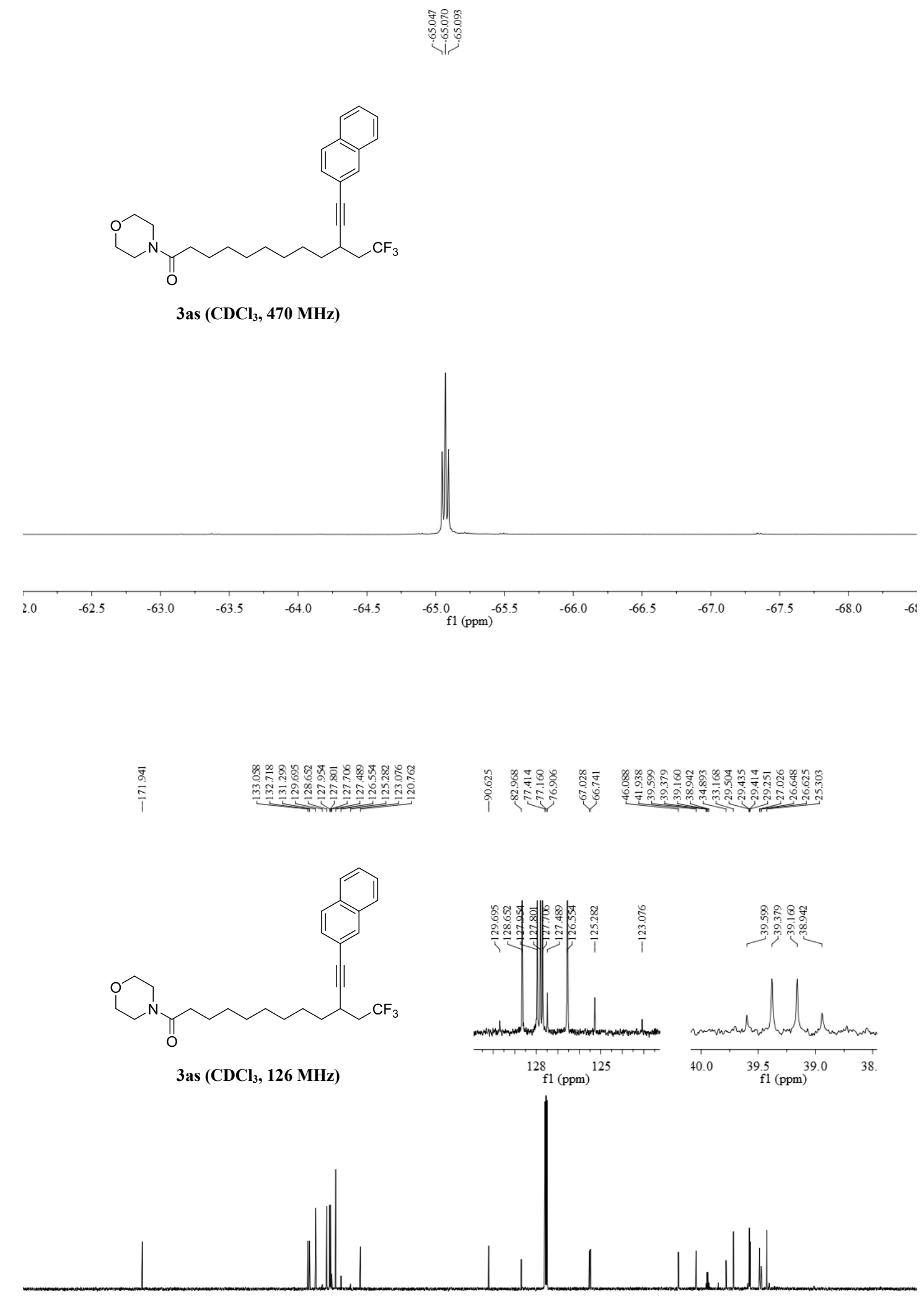

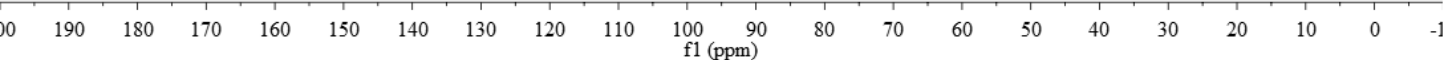




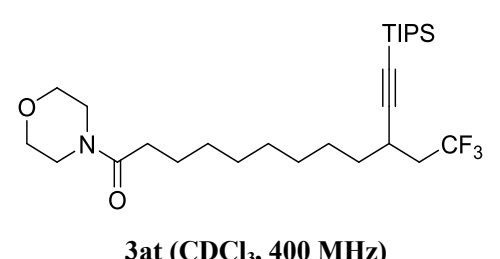

3at $\left(\mathrm{CDCl}_{3}, 400 \mathrm{MHz}\right)$

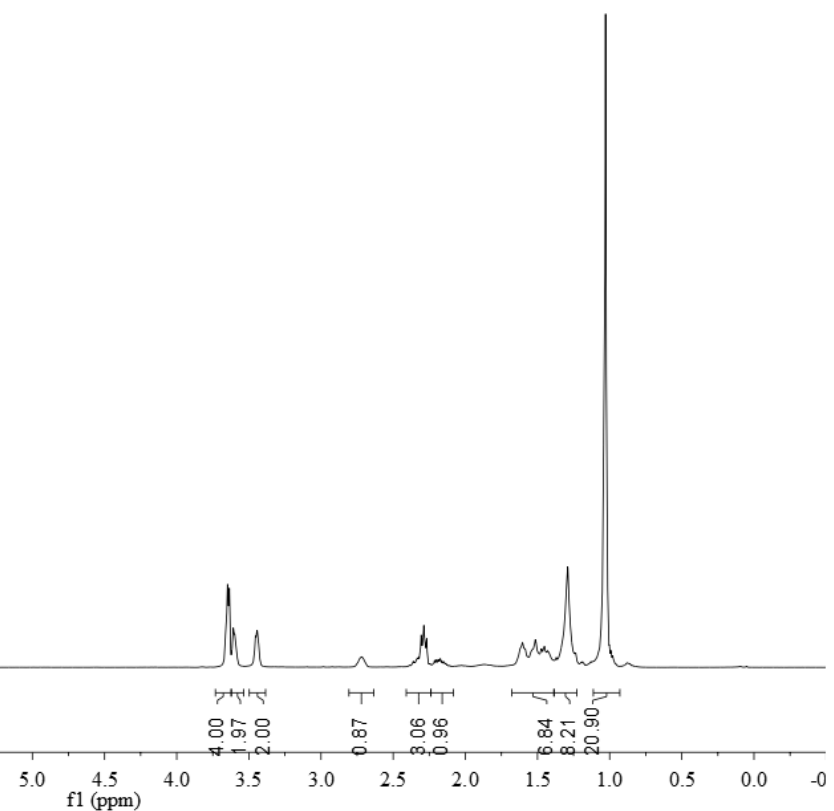

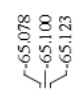

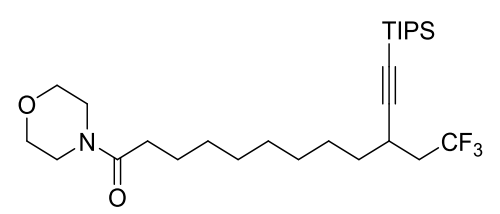

3at $\left(\mathrm{CDCl}_{3}, 101 \mathrm{MHz}\right)$

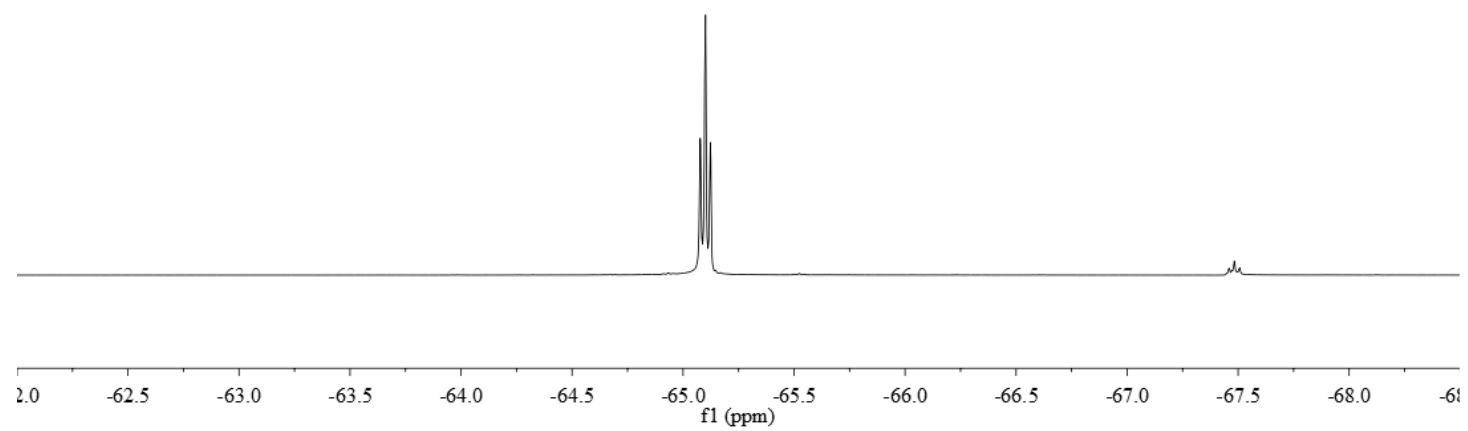



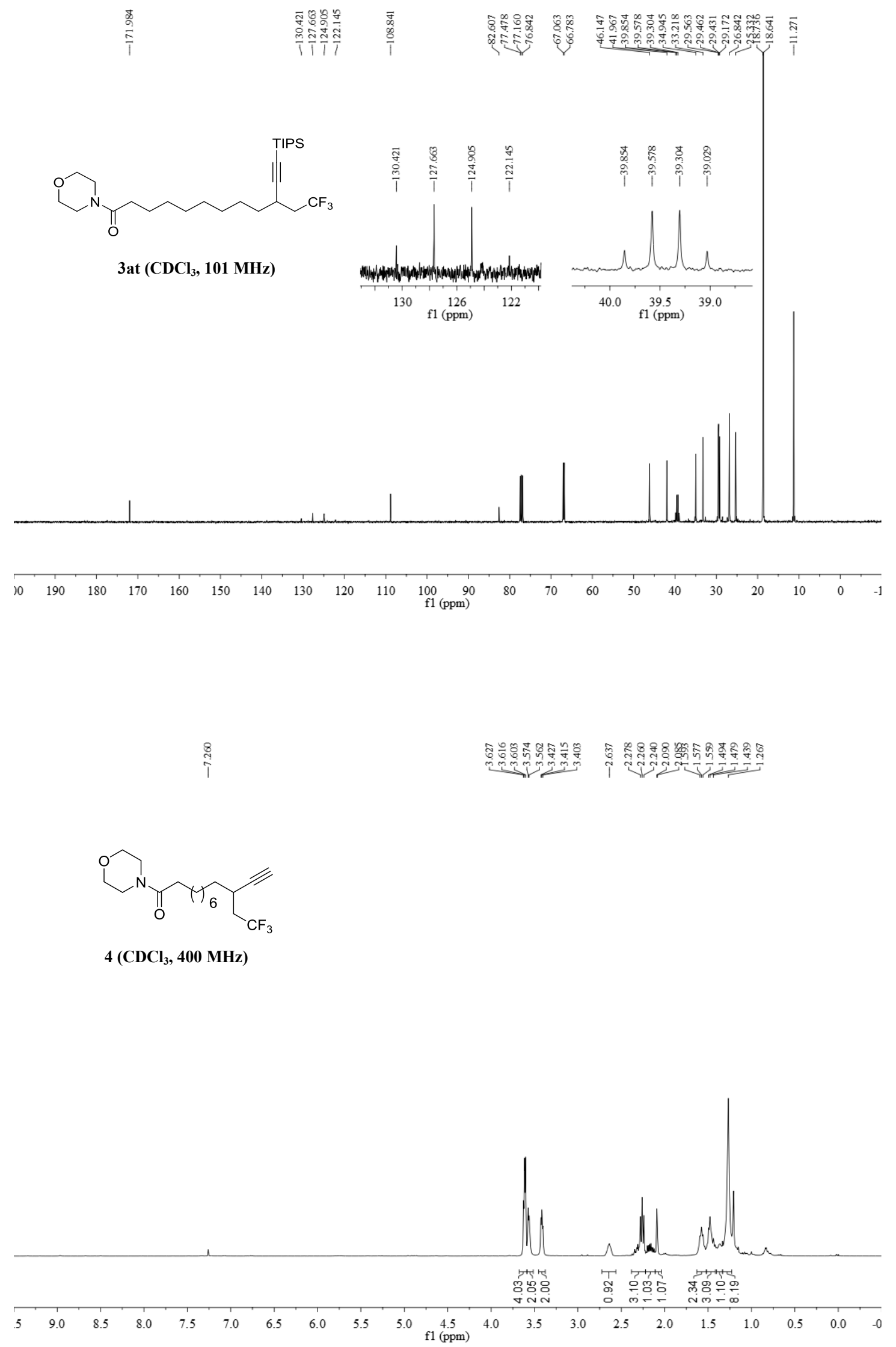


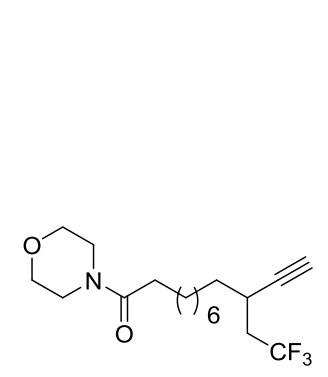

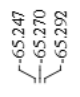

$4\left(\mathrm{CDCl}_{3}, 470 \mathrm{MHz}\right)$
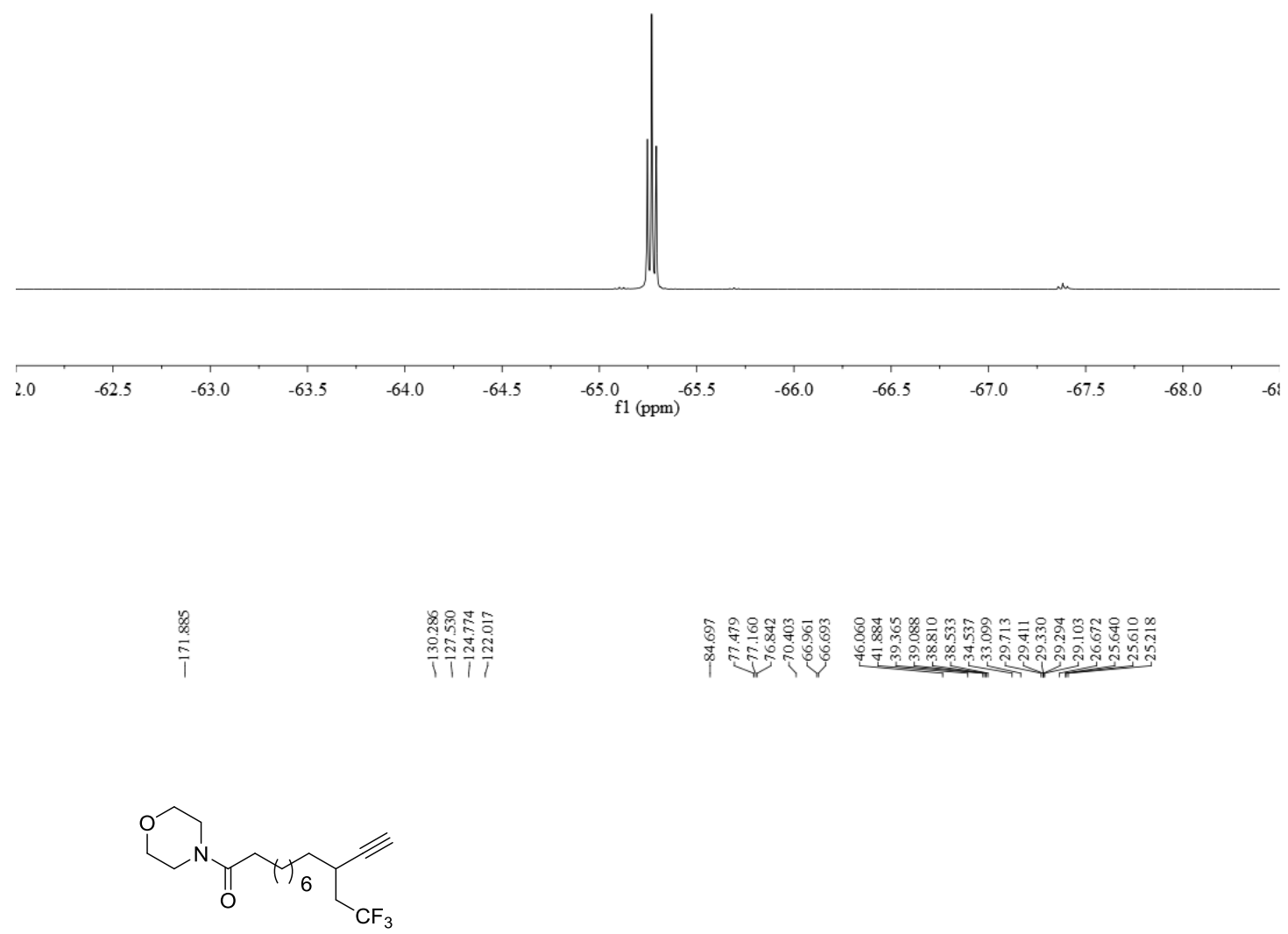

$4\left(\mathrm{CDCl}_{3}, 101 \mathrm{MHz}\right)$

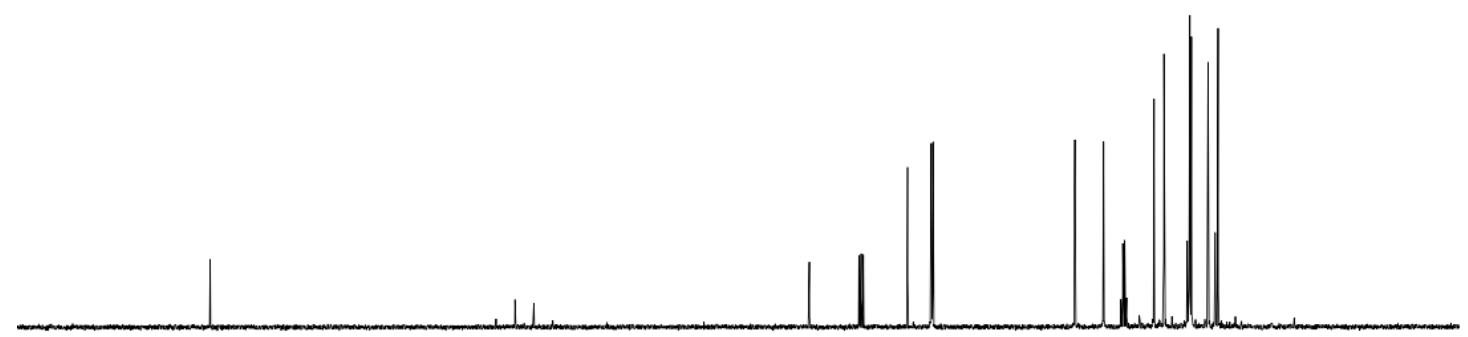

$\begin{array}{lllllllllllllllllllllllllllllllllll}10 & 190 & 180 & 170 & 160 & 150 & 140 & 130 & 120 & 110 & 100 & 90 & 80 & 70 & 60 & 50 & 40 & 30 & 20 & 10 & 0 & -1\end{array}$ 


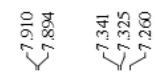

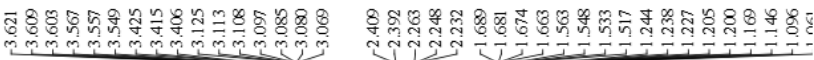

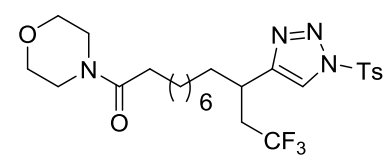

$5\left(\mathrm{CDCl}_{3}, 500 \mathrm{MHz}\right)$
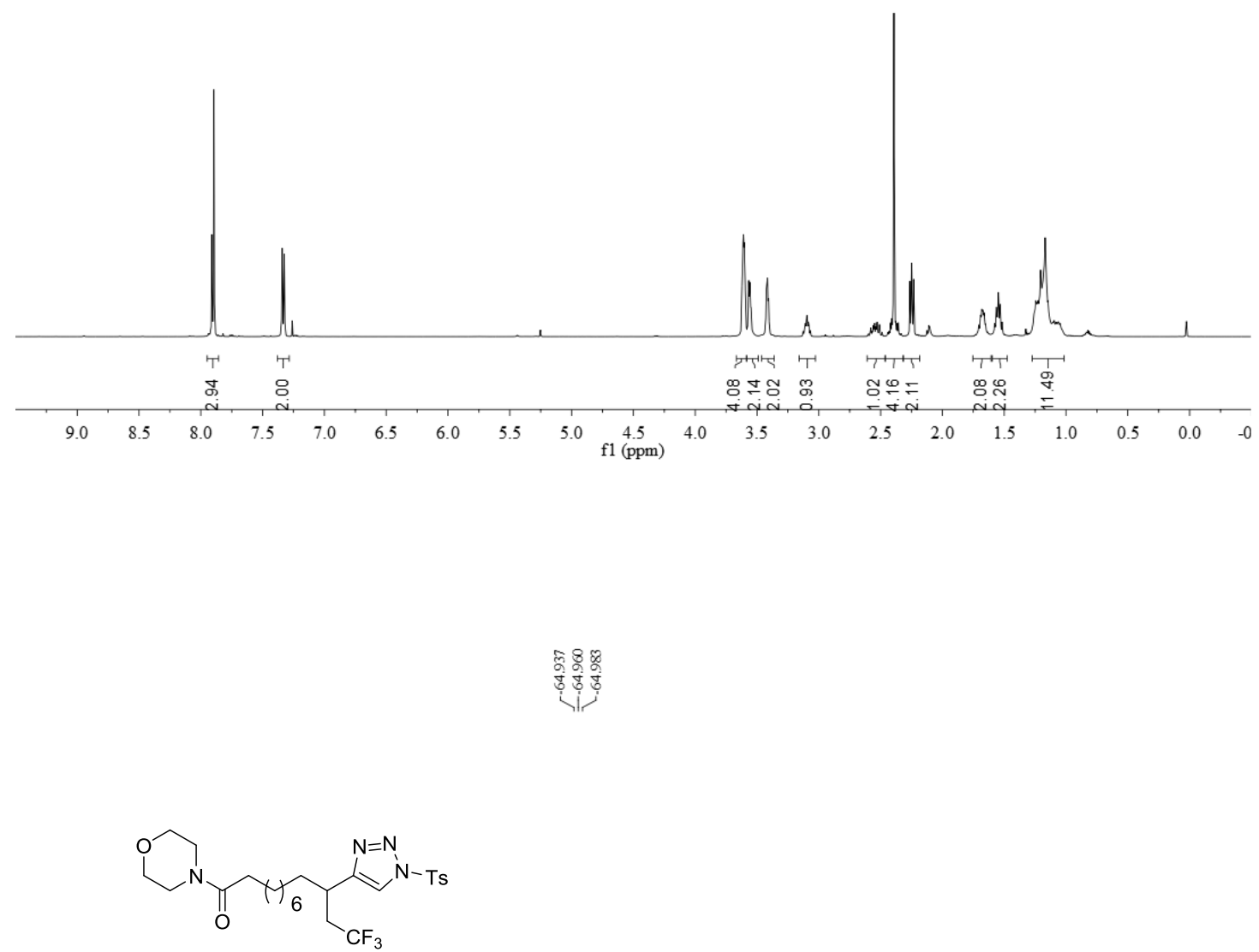

$5\left(\mathrm{CDCl}_{3}, 470 \mathrm{MHz}\right)$

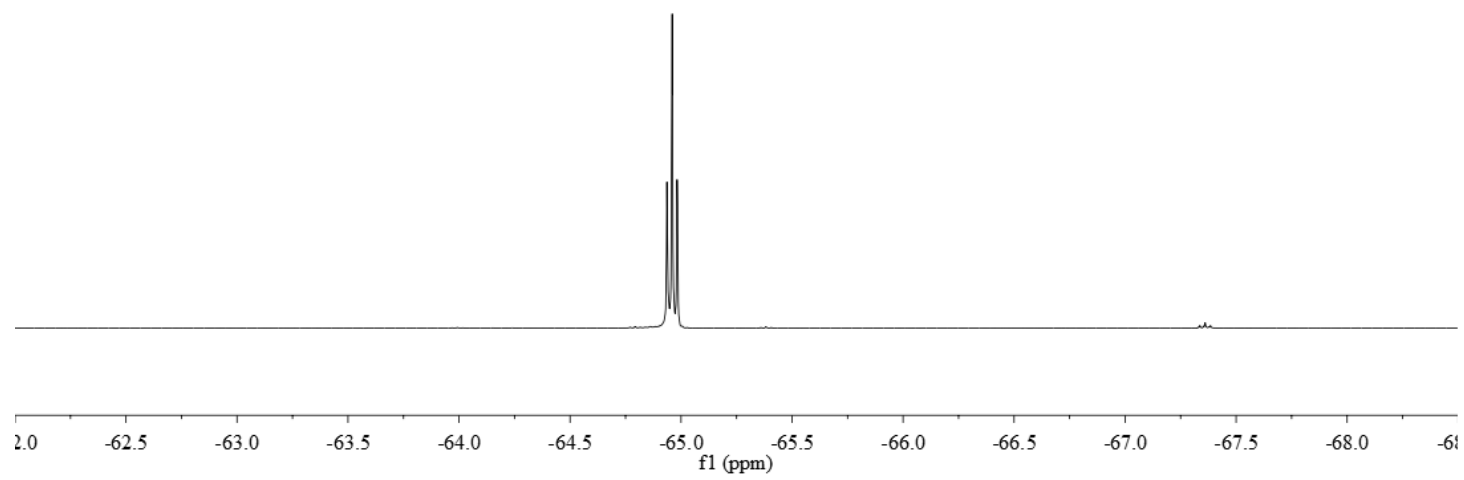




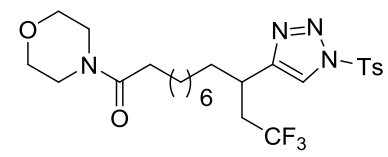

$5\left(\mathrm{CDCl}_{3}, 126 \mathrm{MHz}\right)$

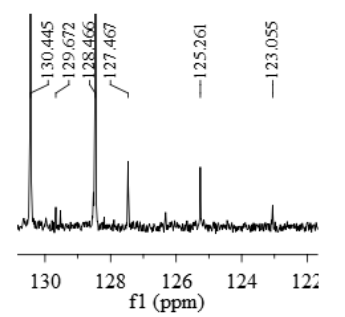

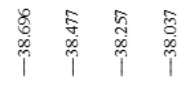

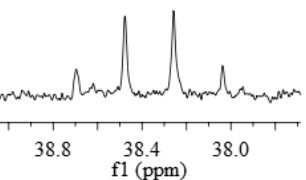

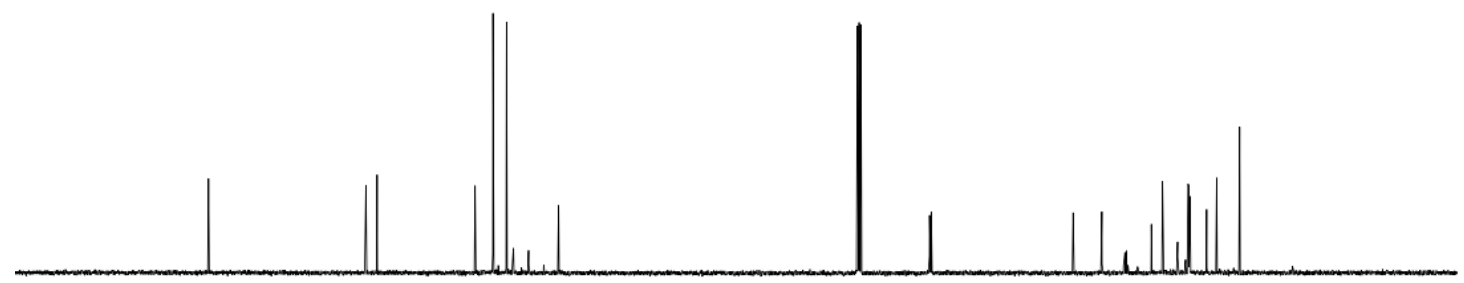

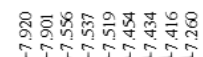

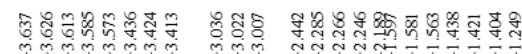

$\begin{array}{lllllllllllllllllllllllllllllllllll}10 & 190 & 180 & 170 & 160 & 150 & 140 & 130 & 120 & 110 & \underset{\mathrm{f} 1(\mathrm{ppm})}{100} & 80 & 70 & 60 & 50 & 40 & 30 & 20 & 10 & 0 & -1\end{array}$<smiles>O=C(CC(=O)N1CCOCC1)CC(CC(=O)c1ccccc1)CC(F)(F)F</smiles>

$6\left(\mathrm{CDCl}_{3}, 400 \mathrm{MHz}\right)$

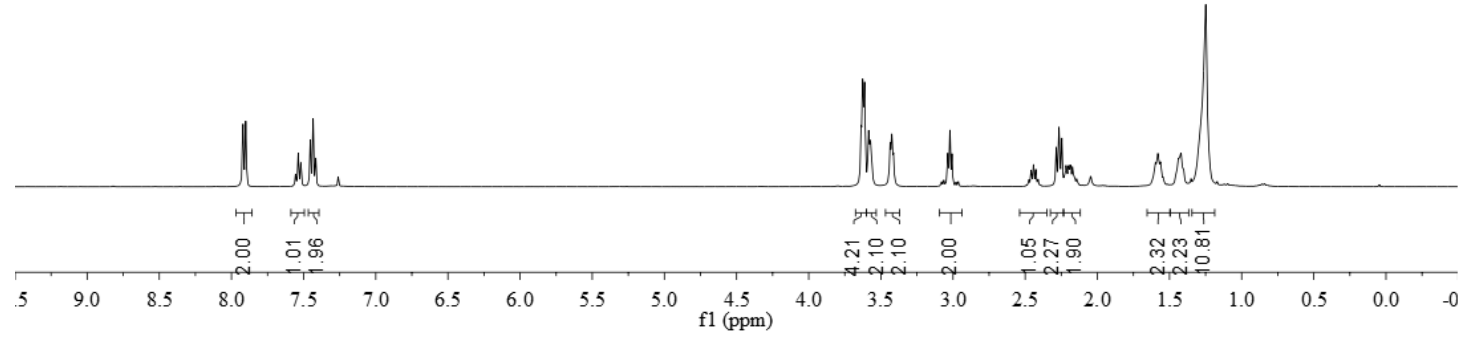




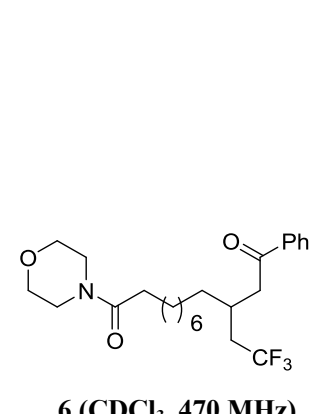

$6\left(\mathrm{CDCl}_{3}, 470 \mathrm{MHz}\right)$
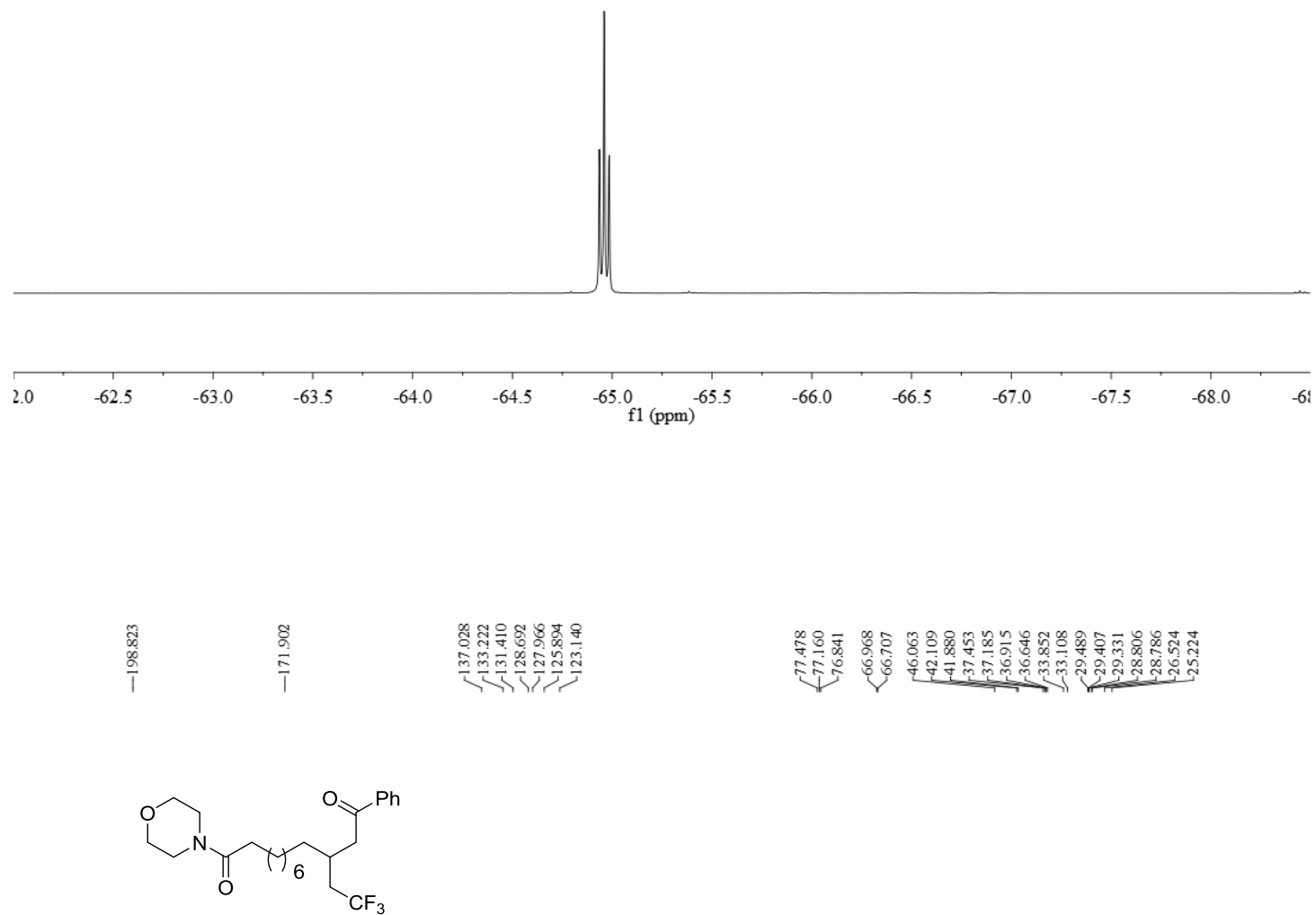

$6\left(\mathrm{CDCl}_{3}, 101 \mathrm{MHz}\right)$

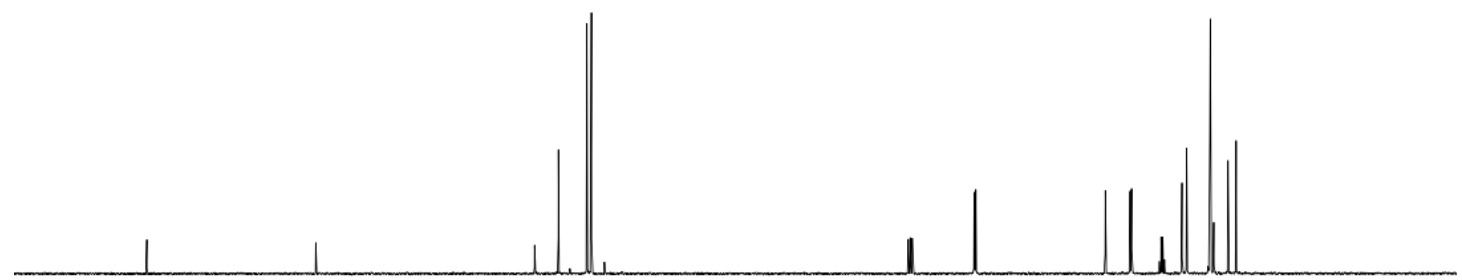

$\begin{array}{llllllllllll}20 & 210 & 200 & 190 & 180 & 170 & 160 & 150 & 140 & 130 & 120 & \begin{array}{l}110 \\ \mathrm{f} 1(\mathrm{ppm})\end{array}\end{array}$ 


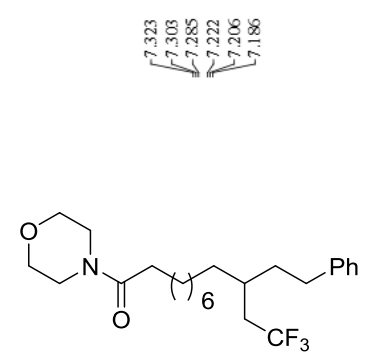

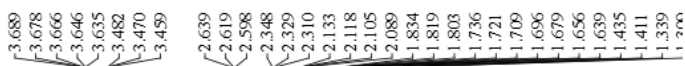

$7\left(\mathrm{CDCl}_{3}, 400 \mathrm{MHz}\right)$
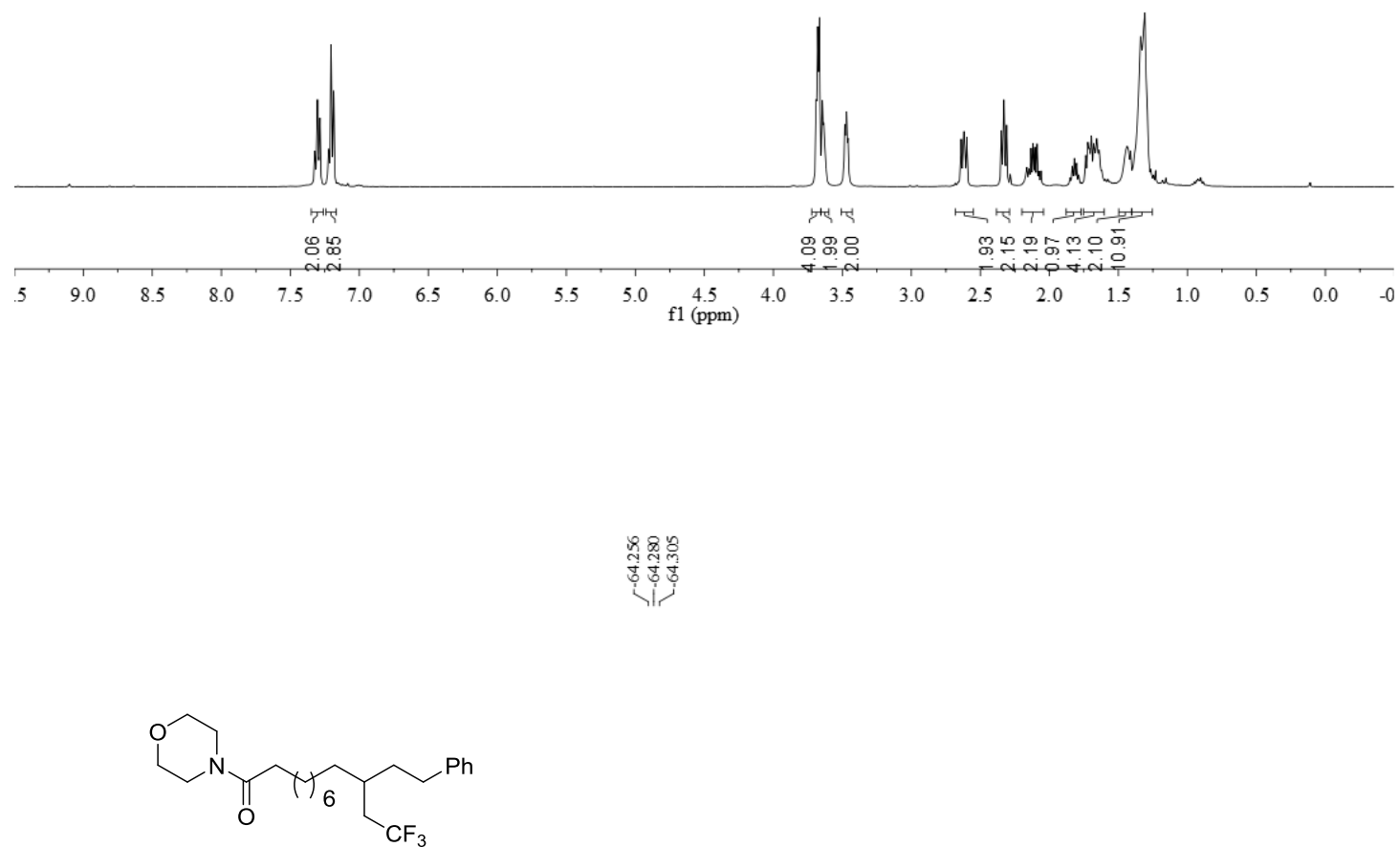

$7\left(\mathrm{CDCl}_{3}, 470 \mathrm{MHz}\right)$

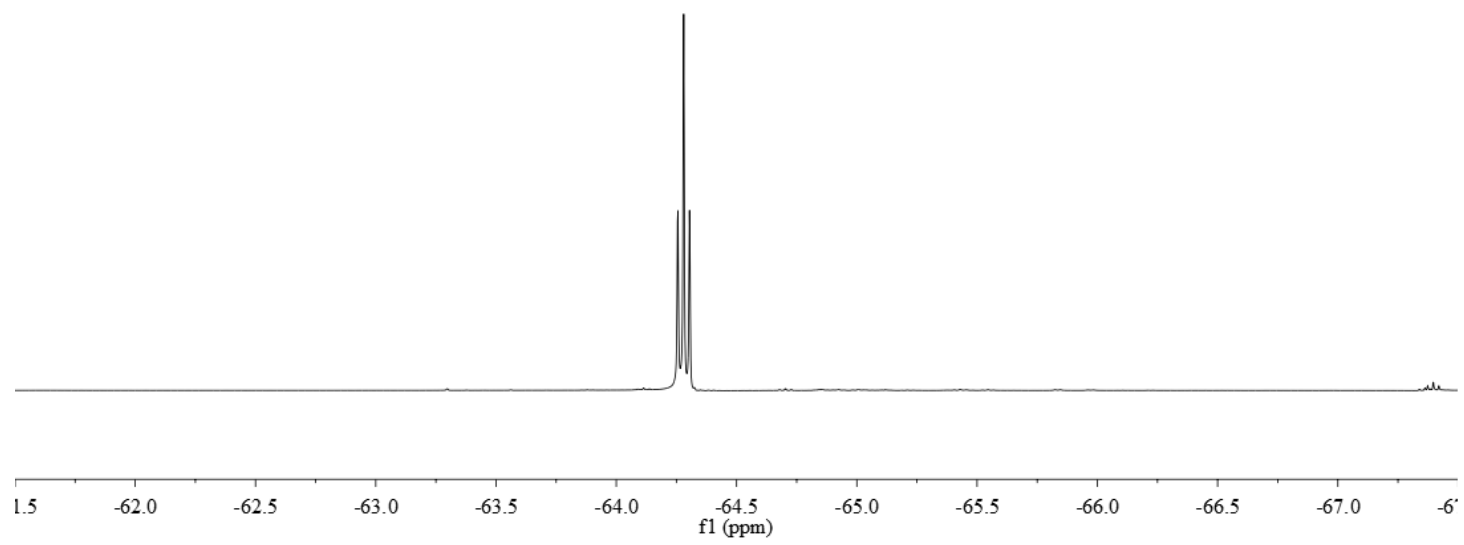



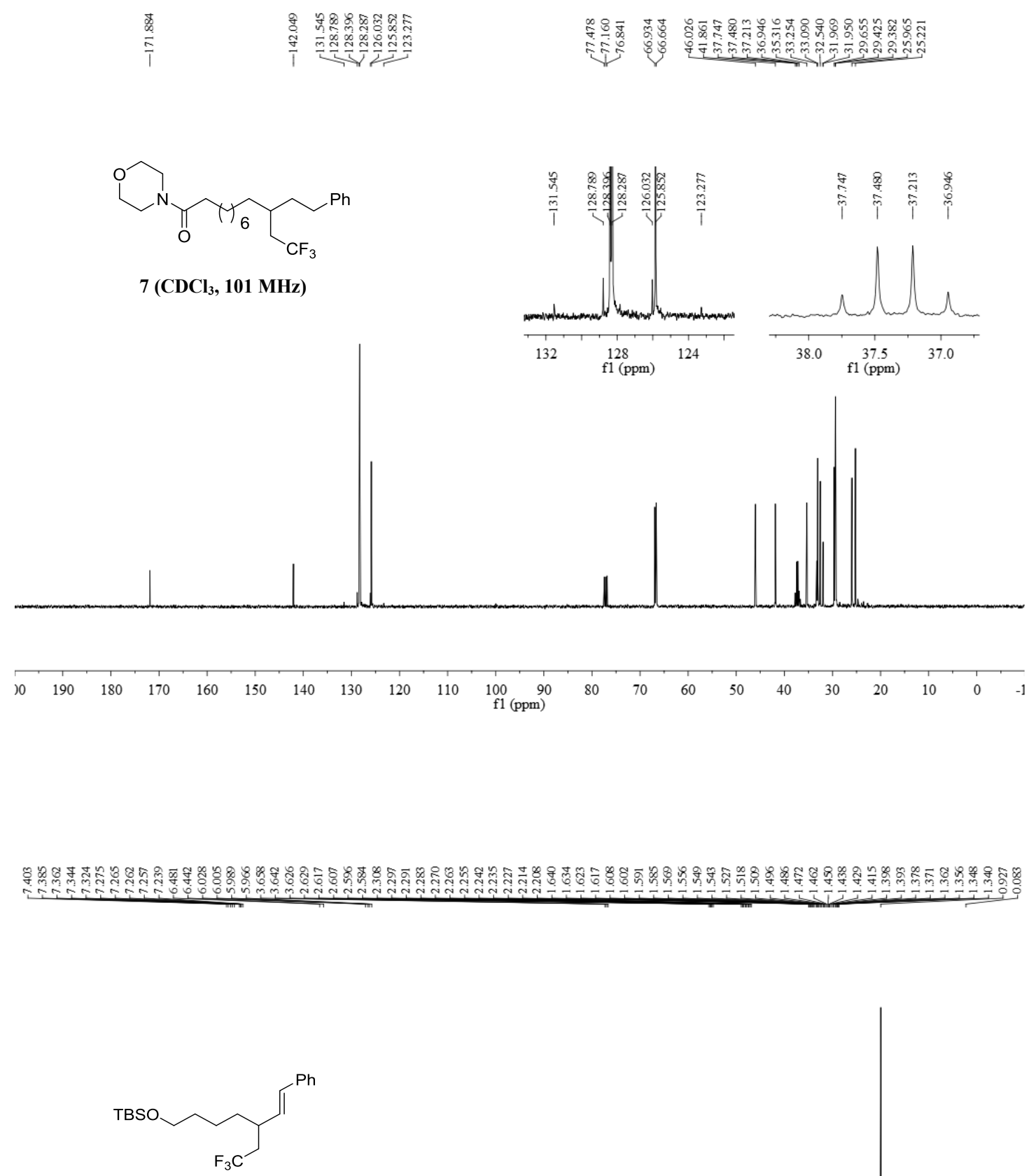

$8\left(\mathrm{CDCl}_{3}, 400 \mathrm{MHz}\right)$

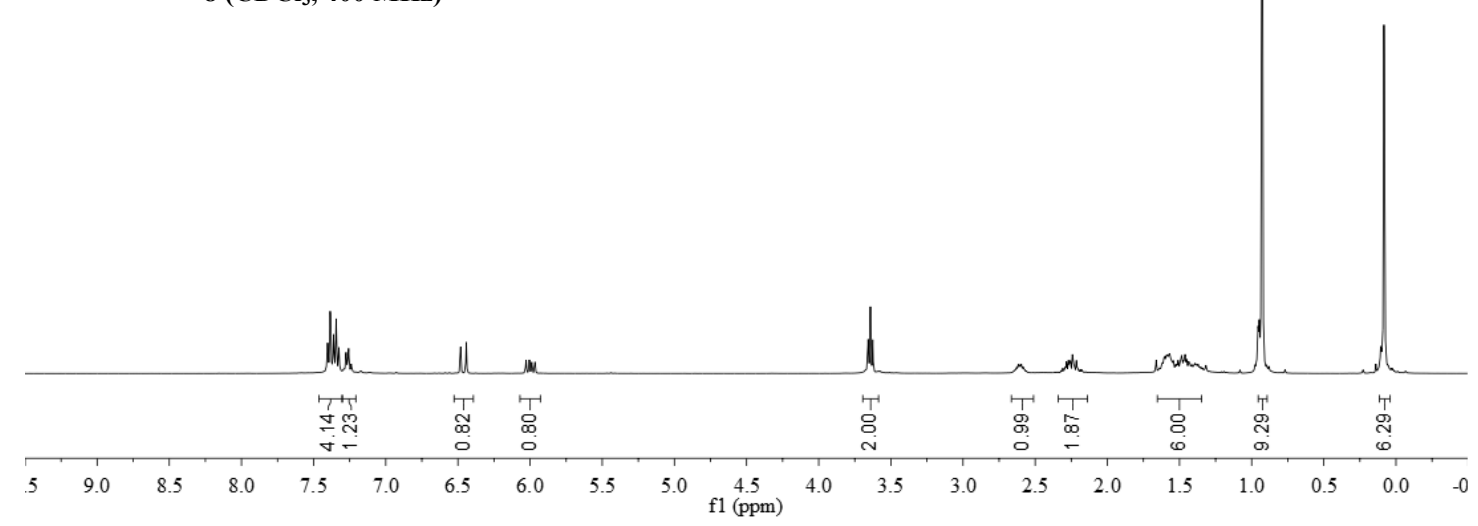




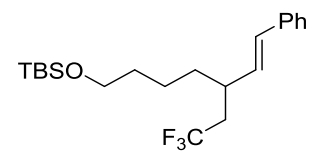

$8\left(\mathrm{CDCl}_{3}, 470 \mathrm{MHz}\right)$

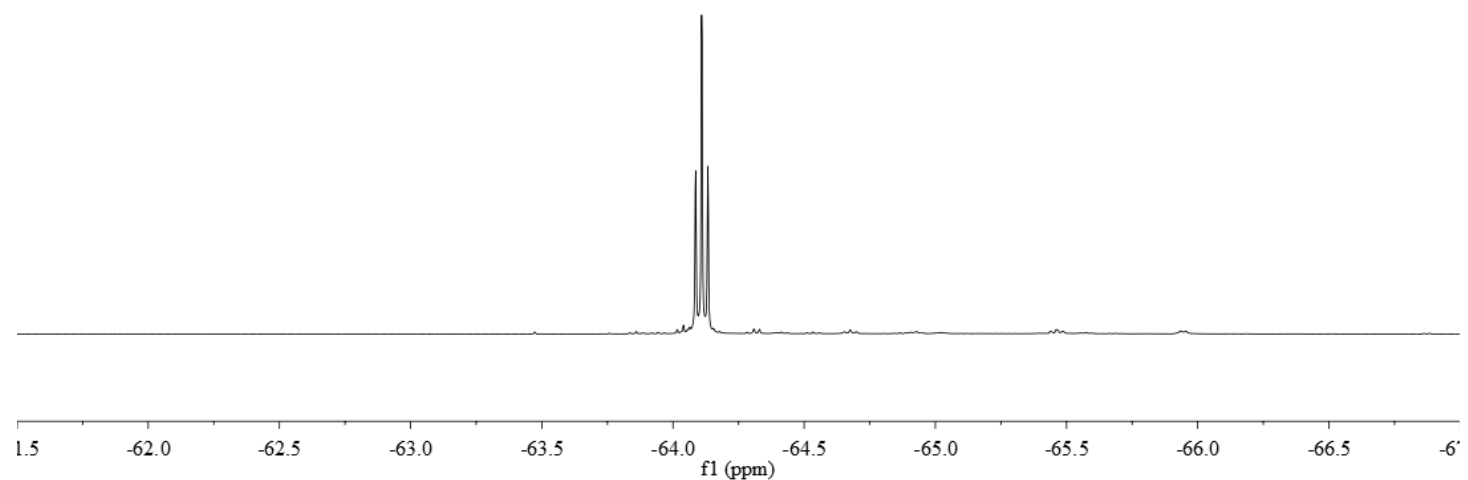

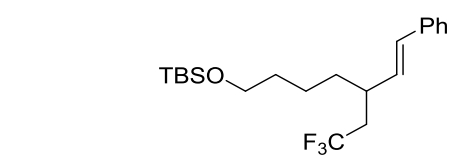

$8\left(\mathrm{CDCl}_{3}, 101 \mathrm{MHz}\right)$

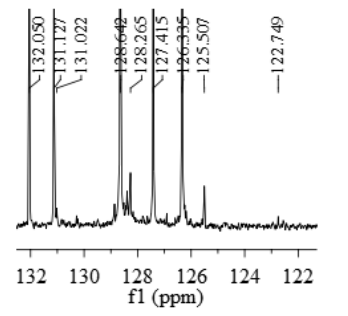

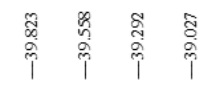

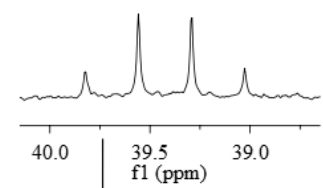

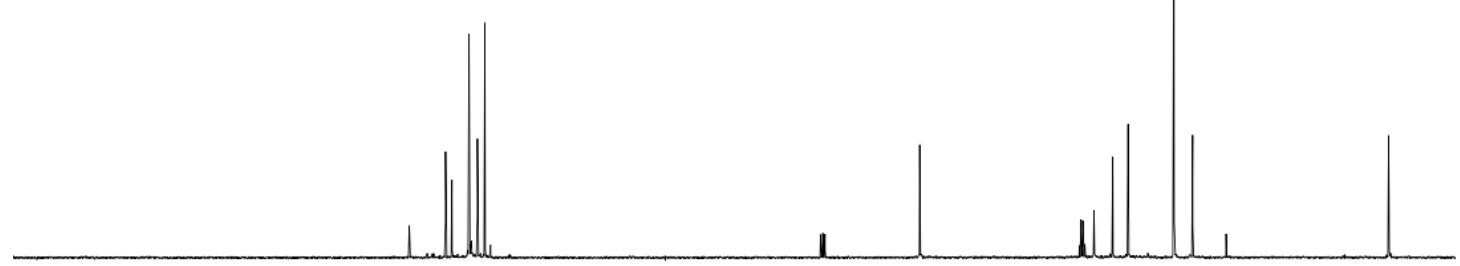

190

$90 \quad 180 \quad 1$ 160 $50 \quad 1$ $130 \quad 120$

110 

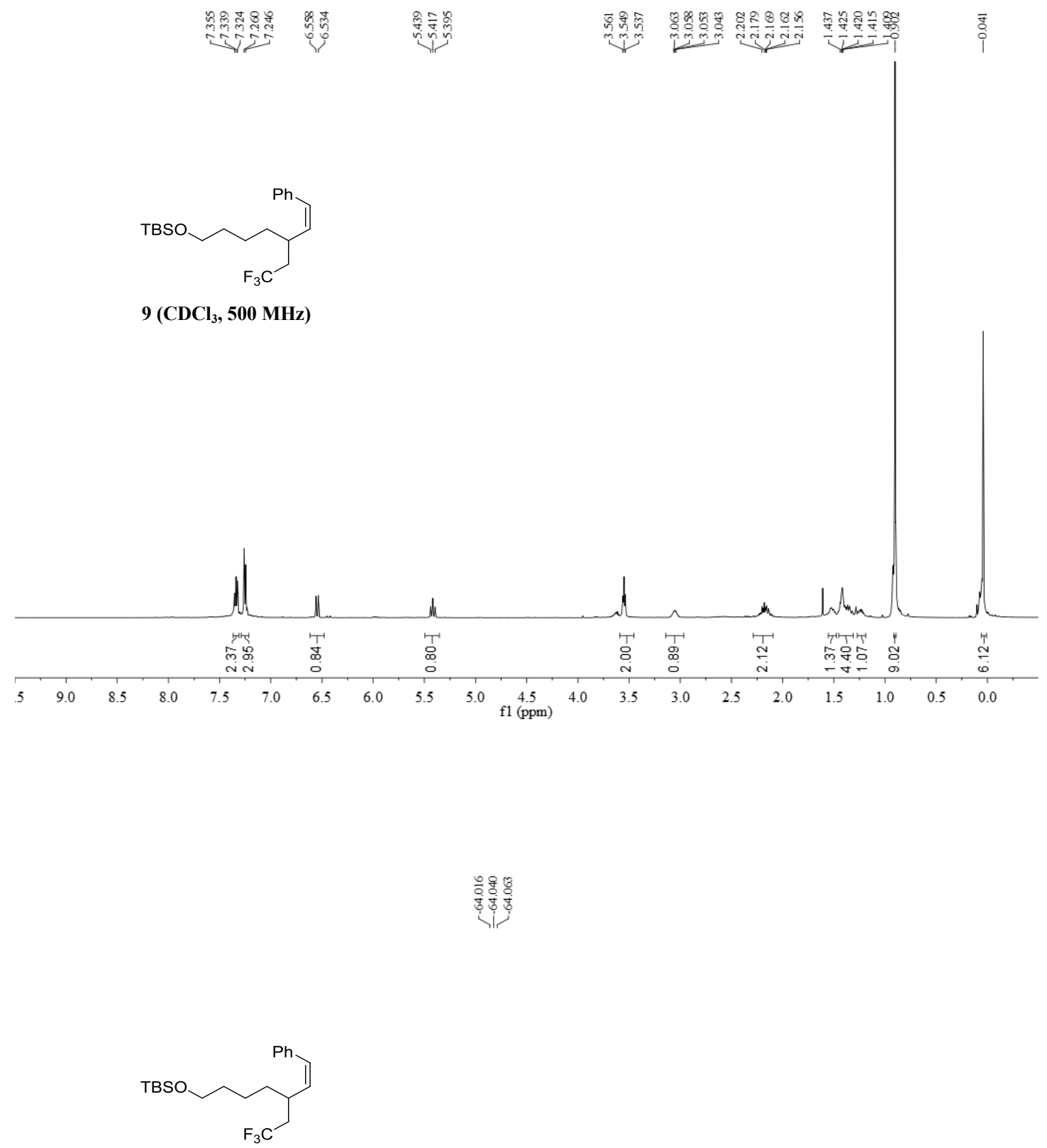

$9\left(\mathrm{CDCl}_{3}, 470 \mathrm{MHz}\right)$

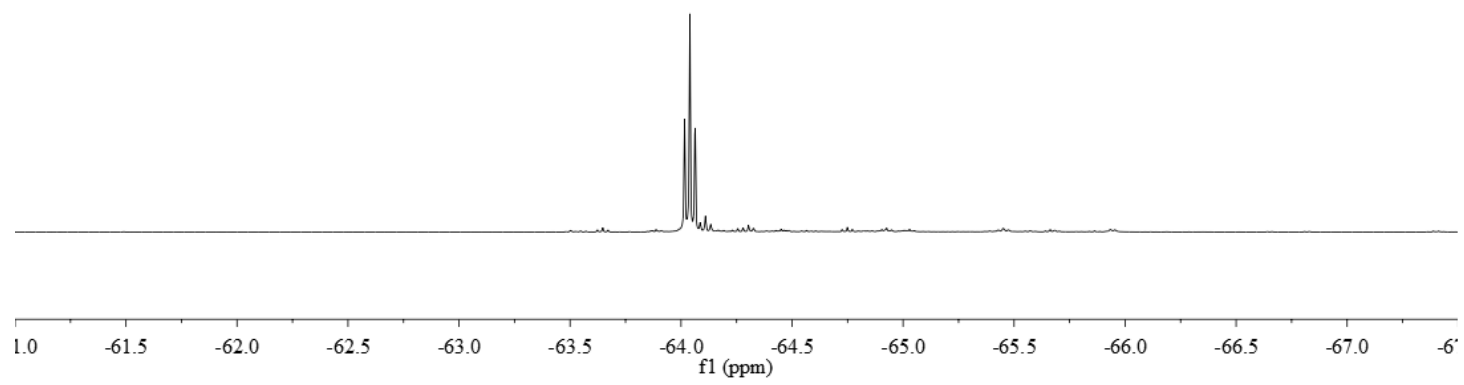




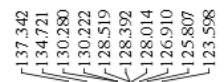

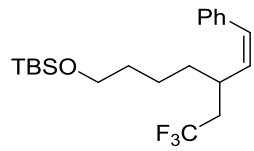

$9\left(\mathrm{CDCl}_{3}, 126 \mathrm{MHz}\right)$
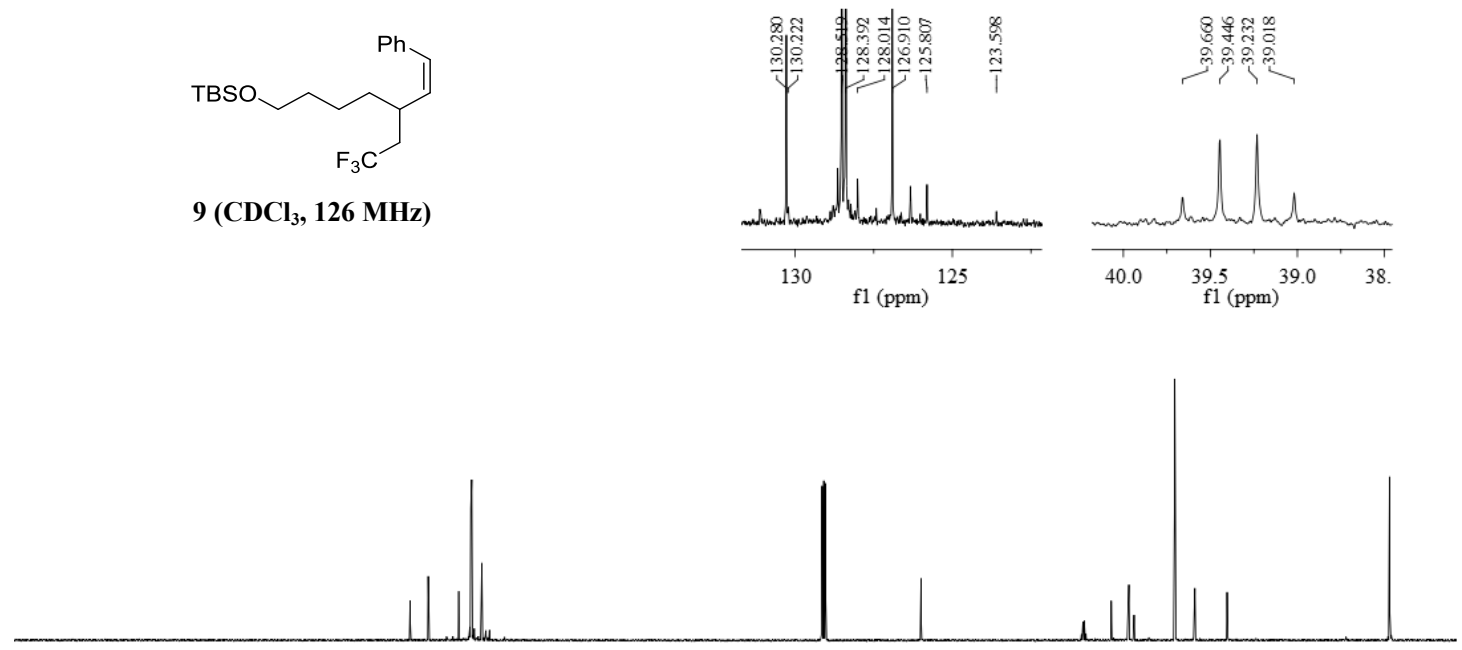

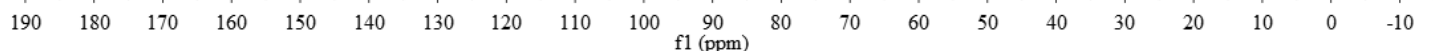

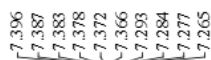

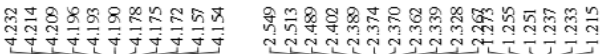

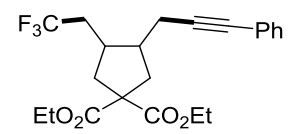

$11\left(\mathrm{CDCl}_{3}, 400 \mathrm{MHz}\right)$

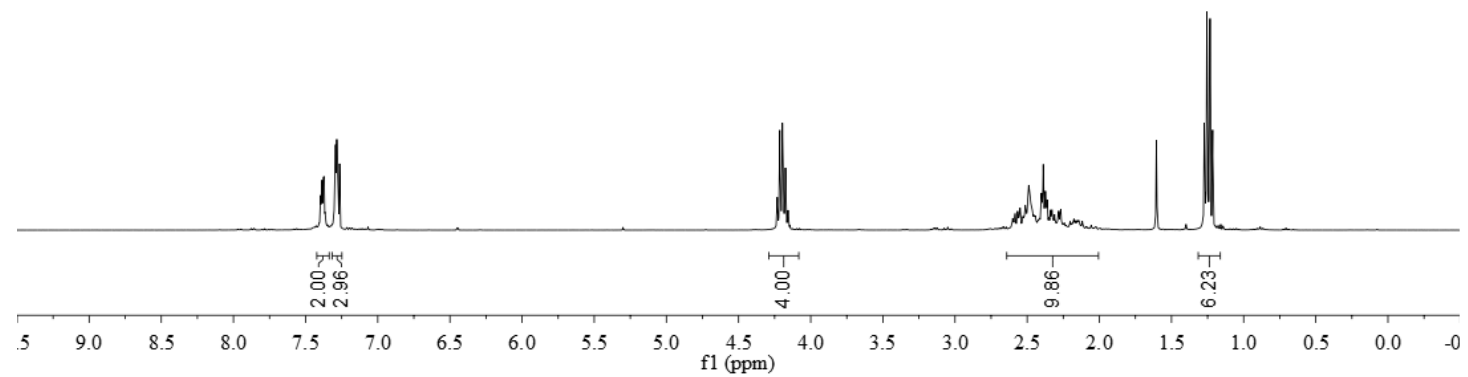




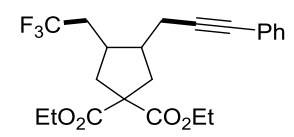

$11\left(\mathrm{CDCl}_{3}, 470 \mathrm{MHz}\right)$
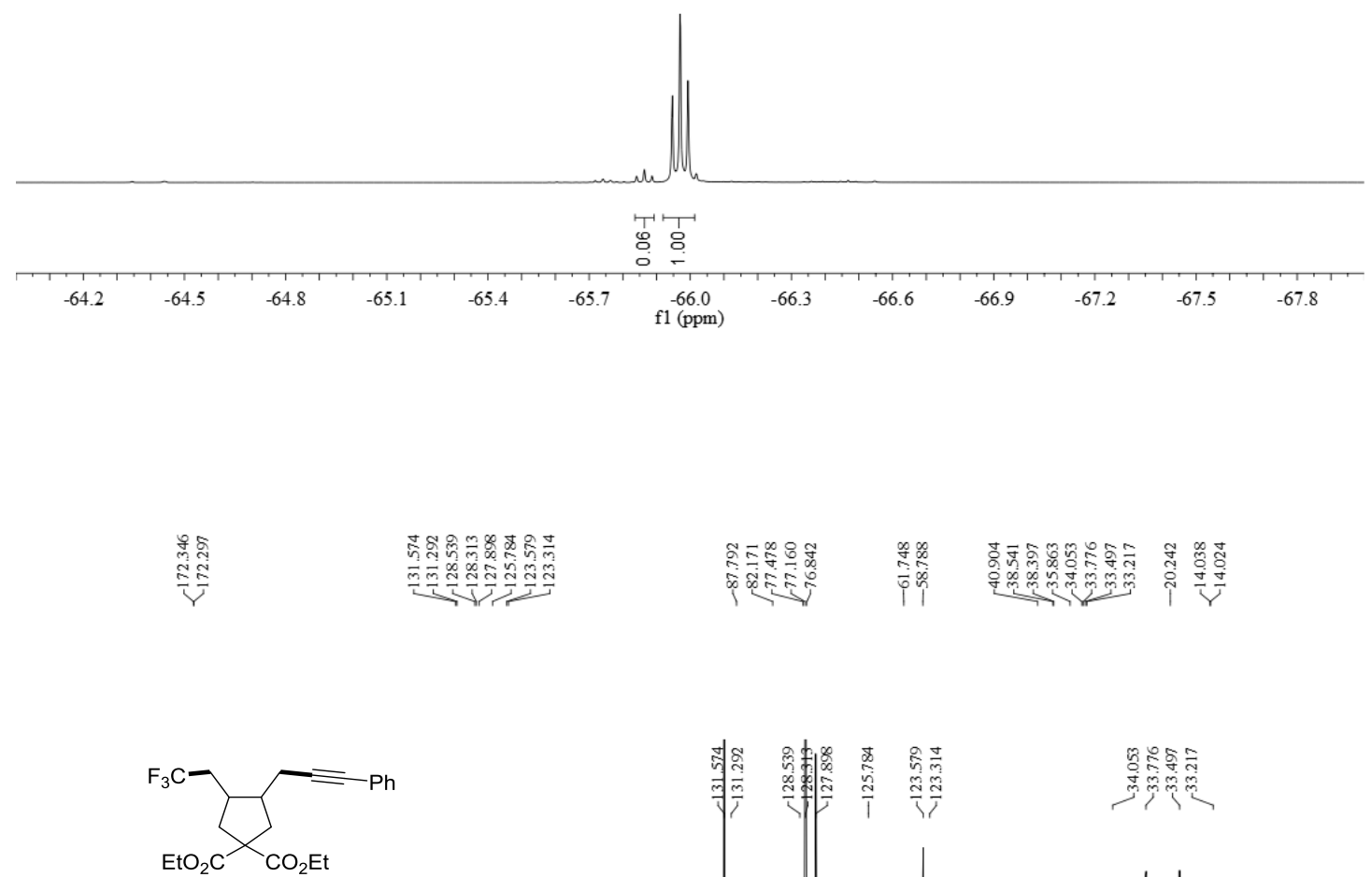

$11\left(\mathrm{CDCl}_{3}, 101 \mathrm{MHz}\right)$
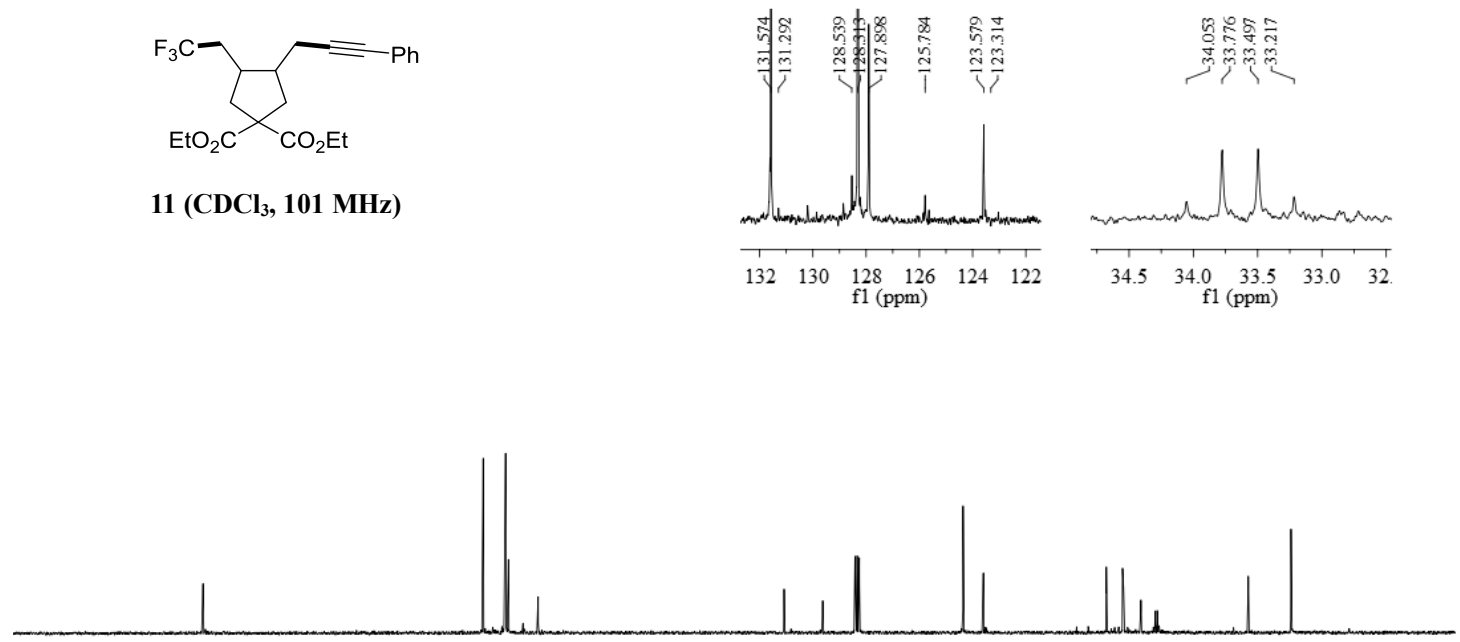

$\begin{array}{lllllllllll}10 & 190 & 180 & 170 & 160 & 150 & 140 & 130 & 120 & 110 & \begin{array}{c}100 \\ \mathrm{f} 1(\mathrm{ppm})\end{array}\end{array}$ 


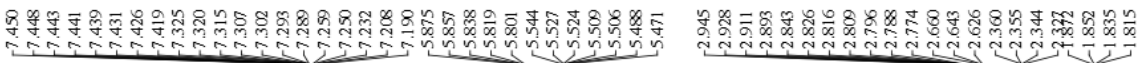<smiles>FC(F)(F)CC=CCC(C=Cc1ccccc1)Cc1ccccc1</smiles>

$13\left(\mathrm{CDCl}_{3}, 400 \mathrm{MHz}\right)$

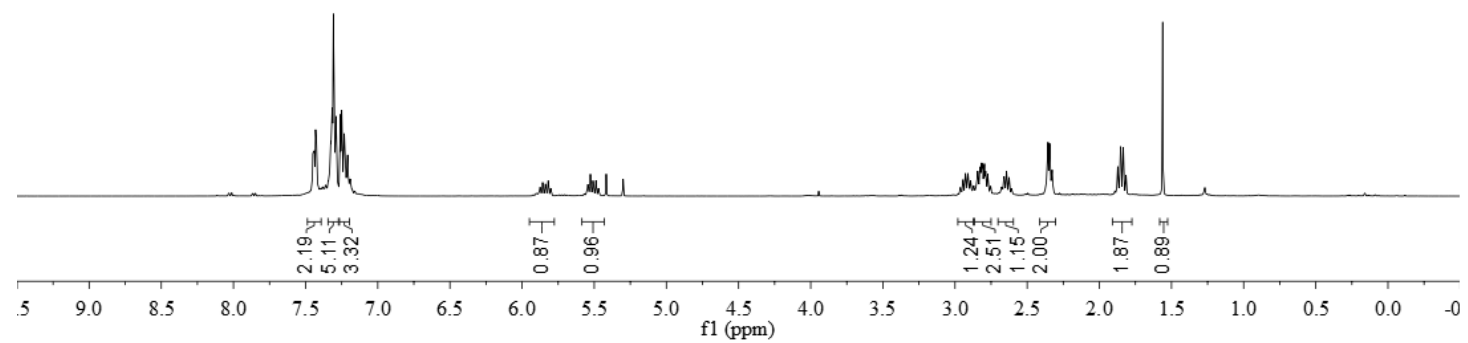

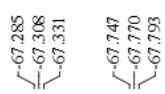

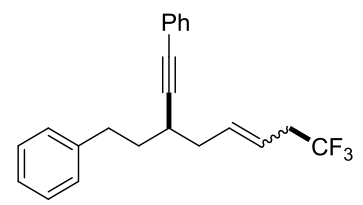

$13\left(\mathrm{CDCl}_{3}, 470 \mathrm{MHz}\right)$

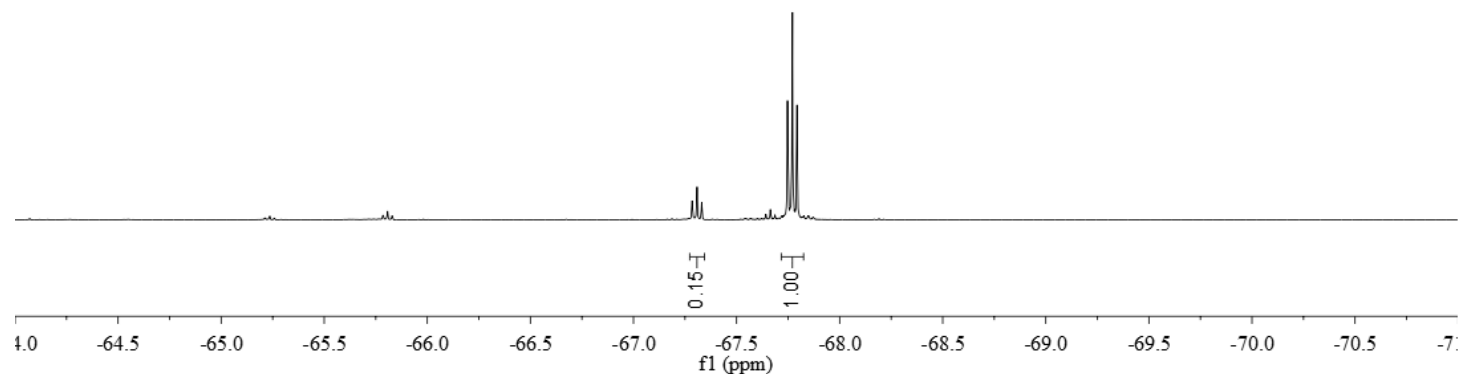




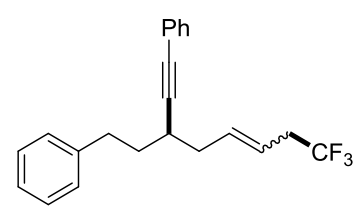

$13\left(\mathrm{CDCl}_{3}, 101 \mathrm{MHz}\right)$
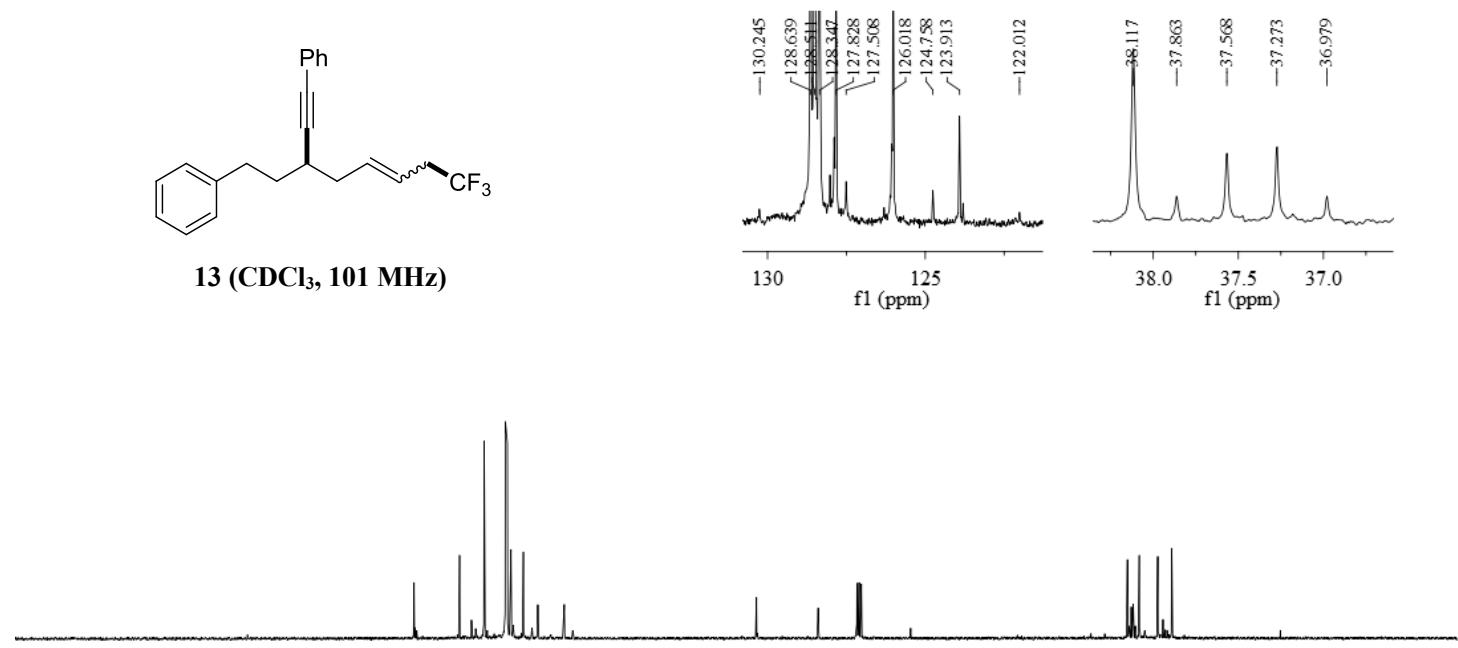

$\begin{array}{llllllllllllllllllllllllllll}10 & 190 & 180 & 170 & 160 & 150 & 140 & 130 & 120 & 110 & 100 & 90 & 80 & 70 & 60 & 50 & 40 & 30 & 20 & 10 & 0 & -1\end{array}$ 